\title{
CONTEXT-FREE GRAMMAR FORMS
}

We initiate the study of form theory by considering contextfree grammar forms. As will be seen many of the reduction and normal form results for context-free grammars also hold for context-free grammar forms. These results together with some stronger ones enable us to obtain inductive proofs for closure results in the case of g-interpretations. Moreover these normal forms also enable the proof of principality of the g-grammatical families to be carried out by induction as we11. These results are found in Sections 11.1, II.2 and II.4. In Section II.3 the study of collections of grammar families is initiated leading to the notion of a production minimal grammar form and the effectiveness of its construction. Section II.5 is concerned with syntax analysis and pushdown acceptor forms, while Section II.6 is concerned with collections of grammatical families and some decidability results.

We use the notation $\mathcal{L}(F I N), \mathcal{L}$ (REG), $\mathcal{L}$ (LIN) and $\mathcal{L}$ (CF) to denote the families of finite, regular, linear and context-free languages, respectively.

Bibliographic and historical comments are to be found in Chapter $V$. 


\section{II.1 The Basics of Context-Free Grammar Forms}

This section introduces the two kinds of interpretation upon which most of grammar form theory is based. This is followed by a number of examples not only to give the reader some insight into these ideas but also to give some motivation for the choice of the particular interpretations that have been mainly investigated up until now. Finally the notions of the grammar and grammatical families defined by a grammar form are introduced. We also relate interpretations to grammar morphisms of a particular kind.

Throughout this book recall that we use the following useful convention (the $\lambda$-convention).

\section{Convention}

Given two languages $L_{1}$ and $L_{2}$ we say they are equal modulo $\lambda$ if $L_{1}-\{\lambda\}=L_{2}-\{\lambda\}$. Similarly we say two language families $\mathcal{L}_{1}$ and $\mathcal{L}_{2}$ are equal modulo $\lambda$ and the empty set if for every $L_{1}-\{\lambda\} \neq \emptyset$ in $\mathcal{L}_{1}$ there is an $L_{2}$ in $\mathcal{L}_{2}$ such that $L_{1}-\{\lambda\}=L_{2}-\{\lambda\}$ and vice versa.

Essentially this means we ignore the empty set in language families and the empty word in languages.

\section{II.1.1 Two Kinds of Interpretation}

In any study of the literature of form theory the reader will notice that there are two basic definitions of interpretation which have been most studied. These we refer to as the g-and s-interpretations. The g-interpretation is the one first introduced by Ginsburg and his colleagues, while the s-interpretation was first studied in detail for EOL forms by Salomaa and his colleagues (this is also known as a strict interpretation). Since the s-interpretation is more general than the g-interpretation the results we present on context-free grammar forms will where possible be given for s-interpretations.

Before giving the definition of s-interpretation we first need the notion of a disjoint-finite-letter substitution (dfi-substitution). Let $U, V$ be two alphabets and $\mu$ be a (letter) substituti
$U$ into 2 . Then $\mu$ is a dfl-substitution if for all $X, Y$ in $U$, $\mu(X) \cap \mu(Y)=\emptyset$ when $X \neq Y$.

Recall from Section I.1.1 that a context-free grammar is a couple $(G, \Rightarrow)$, where $G=(V, \Sigma, P, S)$ is a production scheme, that is $V-\Sigma$ is the nonterminal alphabet, $\Sigma$ the terminal alphabet, $P \subseteq(V-\Sigma) \times V *$ 
is a finite set of productions and $S$ in $V-\Sigma$ is the sentence symbol. Moreover $\Rightarrow$ is the sequential rewrite relation over $V^{*}$ induced by $P$ (see Section I.1.1 for further details).

Letting $M, N \subseteq V^{*}$ then the notation $M \rightarrow N$ denotes $\{\alpha \rightarrow \beta: \alpha$ is in $M$ and $\beta$ is in $N$ in the following.

Without further ado we are now able to define the two notions of interpretation.

\section{Definition}

Let $G_{i}=\left(V_{i}, \Sigma_{i}, P_{i}, S_{i}\right) i=1,2$ be two grammars. We say $G_{2}$ is an s-interpretation of $G$ modulo $\mu$, denoted $G_{2} \underset{s}{A} G_{1}(\mu)$, where $\mu$ is a dfl-substitution on $V_{1}^{*}$, if conditions (i) through (iv) obtain:

(i) $\mu(A) \subseteq V_{2}-\Sigma_{2}$, for al1 A in $V_{1}-\Sigma_{1}$,

(ii) $\mu($ a $) \subseteq \Sigma_{2}$, for all a in $\Sigma_{j}$,

(iii) $P_{2} \subseteq \mu\left(P_{1}\right)$, where $\mu\left(P_{1}\right)=\underbrace{}_{A \rightarrow \alpha \text { in } P_{1}} \mu(A \rightarrow \alpha)$,

where $\mu(A \rightarrow \alpha)=\mu(A) \rightarrow \mu(\alpha)$, and

(iv) $s_{2}$ is in $u\left(s_{1}\right)$.

The definition of g-interpretation is very similar except that terminal letters can be replaced by sets of terminal words rather than just by sets of terminal letters. This notion is defined as follows.

\section{Definition}

Let $G_{i}=\left(V_{i}, \Sigma_{i}, P_{i}, S_{i}\right), i=1,2$ be two grammars. We say $G_{2}$ is a g-interpretation of $G_{1}$ modulo $\mu$, denoted $G_{2} \Delta G_{1}(\mu)$, where $\mu$ is a (finite) substitution on $V_{T}^{*}$, if conditions (i) through (iv) obtain:

(i) $\mu$ is a dfl-substitution from $V_{1}-\Sigma_{1}$ into $V_{2}-\Sigma_{2}$,

(ii) $\mu(a) \subseteq \Sigma_{2}^{*}$, for all a in $\Sigma_{\eta}$,

(iii) $P_{2} \subseteq \mu\left(P_{1}\right)$, where $\mu\left(P_{1}\right)=\underbrace{}_{A \rightarrow \alpha \text { in } P_{1}} \mu(A) \rightarrow \mu(\alpha)$, and

(iv) $S_{2}$ is in $\mu\left(s_{1}\right)$.

In both cases $G_{T}$ is the source, master or form grammar, while $G_{2}$ is the image or interpretation grammar. Operationally we obtain s-interpretation grammars from $G_{1}$ by mapping distinct terminals into disjoint sets of terminals and distinct nonterminals into disjoint sets of nonterminals. Productions in the s-interpretation grammar are obtained as images of the productions in the form grammar $G_{1}$, while the sentence symbol is an image of $s_{1}$. We obtain g-interpretation grammars in a similar way except that terminals are mapped into sets of terminal words rather than terminal letters. One immediate result 
of this is that s-interpretations are necessarily length preserving while g-interpretations are not. Secondly, it means that every s-interpretation grammar is a g-interpretation grammar but the converse does not hold.

Whenever $P_{2}=\mu\left(P_{1}\right)$ we say that $G_{2}$ is a full s-or ginterpretation of $G_{7}$, written $G_{2} \underset{f s}{\rightarrow} G_{1}$ or $G_{2} \underset{f g}{\vec{G}} G_{1}$, respectively. We now consider some examples to give insight into s-and g-interpretations.

\section{Convention}

We often define grammars by simply listing their productions, in which case we use S, possibly subscripted, to denote the sentence symbol, early upper case Roman letters to denote nonterminals and early lower case Roman letters to denote terminals.

\section{Example 1.1}

Let $G_{1}: S \rightarrow$ a be the form grammar.

Then $F: S_{F} \rightarrow b ; S_{F} \rightarrow c$ is an $s$-interpretation of $G_{1}$. Let $\mu(S)=\left\{S_{F}\right\}$ and $\mu(a)=\{b, c\}$ then $\mu(S \rightarrow a)=\left\{S_{F} \rightarrow b, S_{F} \rightarrow c\right\}$ hence $F$ is also an fs-interpretation of $G_{\eta}$. Hence $F \vec{s} G_{j}(\mu)$, $F \underset{f s}{\Delta} G_{1}(\mu), F \vec{g} G_{1}(\mu)$ and $F \underset{f g}{a} G_{1}(\mu)$ by the remarks above.

Consider $H: S_{H} \rightarrow a^{5} b ; S_{H} \rightarrow \lambda ; A \rightarrow b b$. Then $H$ is not an s-interpretation of $G_{1}$ since it cannot be obtained by using a dflsubstitution because such a substitution is length preserving.

However $H \underset{g}{\Delta} G_{1}(\mu)$ where $\mu$ is defined by: $\mu(S)=\left\{A, S_{H}\right\}$, and $\mu(a)=\left\{a^{5} b, b b, \lambda\right\}$ since $P_{H} \subseteq \mu(S \rightarrow a)$. Since $S_{H} \rightarrow b b$ is not in $H$ it follows that $H$ is not an fg-interpretation of $G_{l}$.

Finally let $L$ be any finite language. Then $L$ can be obtained as a g-interpretation of $G_{T}$ (in fact an fg-interpretation) by letting $\mu(S)=\{S\}$ and $\mu(a)=L$

giving a grammar $F$ :

$S \rightarrow x$, for all $x$ in $L$.

clearly $L(G, \Rightarrow)=L$.

\section{Example 1.2}

Let $G_{2}: S \rightarrow S S ; S \rightarrow$ a be the form grammar.

Then we can obtain every context-free language as the language of some s-interpretation and hence g-interpretation of $G_{2}$. (Note that we invoke the $\lambda$-convention here.) 
Consider an arbitrary context-free language $L$. Then it is we 11 known that $L$ can be generated by a context-free grammar in Chomsky Normal Form, that is its productions are either of the type $A^{-} \rightarrow B C$ or $A \rightarrow a$. Let $F=(V, \Sigma, P, Z)$ be such a grammar for $L$ and define $\mu$ by: $\mu(S)=V-\Sigma$ and $\mu(a)=\Sigma$.

Then $P \subseteq \mu(\{S \rightarrow S S, S \rightarrow a\}), Z$ is in $\mu(S)$ and $\mu$ is trivially a dflsubstitution, hence

$F \underset{s}{\Delta} G_{2}(\mu)$.

However it is worth noting the, albeit obvious, fact that there are grammars which are not interpretations of $G_{2}$. Consider $H: S_{H} \rightarrow S_{H} ; S_{H} \rightarrow$ a for example. On the one hand we cannot obtain nonterminals from terminals under either $g$ - or s-interpretation, hence $S_{H} \rightarrow S_{H}$ cannot be an image of $S \rightarrow$ a and on the other hand nonterminals cannot be erased hence $S_{H} \rightarrow S_{H}$ cannot be an image of $S \rightarrow S S$.

\section{Example 1.3}

Let $G_{3}: S \rightarrow a ; S \rightarrow$ aS; $S \rightarrow$ aSS.

As in Example 1.2 it is easy to see that every context-free language can be obtained as the language of some $s$-interpretation of $G_{3}$. This follows from the well known result that every context-free language has a grammar with productions only of the types $A \rightarrow a, A \rightarrow a B$ and $A \rightarrow a B C$. This is known as Greibach 2-standard Normal Form.

Example 1.4

$$
\text { Let } \begin{aligned}
G_{4}: S & \rightarrow a^{i}, 1 \leq i \leq 13 ; \\
S & \rightarrow a^{3} \mathrm{Sa}^{2} \mathrm{Sa}^{7} .
\end{aligned}
$$

Then it will be shown later that we can obtain every context-free language via some interpretation of $G_{4}$. Essentially this follows by observing that $L\left(G_{4}, \Rightarrow\right)=\left\{a^{i}: i \geq 1\right\}$ and that $G_{4}$ is expansive. Such "two-symbol" grammars always give rise to normal form results for the context-free languages. $G_{2}$ and $G_{3}$ are special cases of a much more general "super-normal form" result.

\section{Example 1.5}

We will illustrate with this example some of the effects of relaxing the disjointness condition for nonterminals. Let $G_{5}: S \rightarrow A B$; $S \rightarrow a ; A \rightarrow \lambda ; B \rightarrow \lambda$. It is not difficult to see that as far as languages are concerned we obtain no more than $G_{1}$ under either the $s$ - or g-interpretations (again invoking the $\lambda$-convention). This 
follows by observing that the production $S \rightarrow A B$ can only give. rise to the empty word when used in a derivation.

However if we relax the disjointness condition for nonterminals we could define $\mu$ by:

$$
\mu(a)=\{a\} \text { and } \mu(A)=\mu(B)=\mu(S)=\{S\}
$$

and obtain $G_{2}: S \rightarrow S S ; S \rightarrow$ a as an interpretation of $G_{5}$. Immediately instead of obtaining only finite languages from $G_{5}$ we obtain all contextfree languages! This is called a quasi-interpretation, see section 3.2 .

\section{Example 1.6}

In Examples 1.2 and 1.5 we have observed that nonterminals should not be erased or merged (and under the definition of interpretation cannot be). In this example we demonstrate why we preclude the addition of terminals or nonterminals. from $G_{6}$.

Let $G_{6}: S \rightarrow S$; $\rightarrow$ a, then we can only obtain finite languages

But $F: S \rightarrow$ aS; $S \rightarrow$ a although looking very much like $G_{6}$ gives rise to all regular languages. This is because every regular language can be generated by a right-linear grammar and such a grammar is an interpretation of $F$.

Similarly $G_{2}: S \rightarrow S S$; $S \rightarrow$ a also looks very similar to $G_{6}$ but as we know from Example 1.2 we can obtain all context-free languages from $G_{2}$ !

In both cases we added one symbol from the grammar to one of the productions of the grammar. Again because of the sensitivity of form grammars to such operations the notion of interpretation considered here precludes the introduction of a new symbol where none previously existed. The exception to this is in the treatment of a terminal symbol, for example $H: S \rightarrow$ a is a $g$-interpretation of $G_{1}$, since terminals can expand into terminal words but they cannot be created ex ninizo.

\section{II.1.2 Grammar and Grammatical Families}

We will usually write $G_{1} \underset{g}{a} G_{2}$ or $G_{1} \underset{s}{\Delta} G_{2}$ where $\mu$ is understood. A (context-free) grammar is said to be a (context-free)grammar form if it is used within the framework of interpretations. We assume the existence of countable nonterminal and terminal alphabets in the following. However for simplicity we will assume nonterminal and terminal alphabets are chosen from some common countable "pool" alphabet. This means in particular that a symbol can be either a 
terminal or a nonterminal symbol depending on its context and hence we can avoid the renaming of symbols in some proofs.

The collection of $s$-interpretation grammars derived from a grammar form $G$ is denoted $\xi_{s}(G)$, referred to as the s-grammar family of $G$. Analogously, the collection of 1 anguages obtained from a grammar form $G$ is denoted by $\mathcal{L}_{S}(G, \Rightarrow)$, referred to as the s-grammatical family of $G$, and defined by:

$$
\mathcal{Z}_{S}(G, \Rightarrow)=\left\{L\left(G^{\prime}, \Rightarrow\right): G^{\prime} \underset{S}{\Delta} G\right\} \text {. }
$$

We say $G_{1}$ and $G_{2}$, two gramar forms, are $s$-form equivalent

if $\mathcal{L}_{s}\left(G_{1} \Rightarrow\right)=\mathcal{Z}_{s}\left(G_{2}, \Rightarrow\right)$ and strong $s$-form equivalent if

$\xi_{s}\left(G_{1}\right)=\xi_{s}\left(G_{2}\right)$.

For any grammar form $G, G \underset{S}{\Delta} G$, hence $\vec{S}$ is reflexive. Clearly $\Delta$ is not, in general, symmetric. However since the composition of two dfl-substitutions is a dfl-substitution it follows that the composition of two s-interpretations is an s-interpretation. In other words $\vec{s}$ is transitive and therefore a pre-order.

Consider two grammar forms $G_{1}$ and $G_{2}$ with $G_{1} \underset{s}{\Delta} G_{2}$, then immediately $G_{s}\left(G_{1}\right) \subseteq \xi_{s}\left(G_{2}\right)$ since $s$-interpretation is transitive. Conversely, if $\xi_{s}\left(G_{1}\right) \subseteq \xi_{s}\left(G_{2}\right)$ then $G_{1}$ is in $\xi_{s}\left(G_{2}\right)$ and therefore $G_{1} \vec{s} G_{2}$. It now follows immediately that $\xi_{s}\left(G_{1}\right)=\xi_{s}\left(G_{2}\right)$ iff $G_{1} \vec{S}_{2} G_{2}$ and $G_{2} \underset{s}{\rightarrow} G_{1}$. In section III.5 we show that $\underset{s}{\Delta}$ is decidable, and therefore, that strong $s$-form equivalence is decidable.

Similarly if we base our definitions on g-interpretations we obtain $g_{g}(G)$ the g-grammar family of $G$ and $\mathscr{L}_{g}(G, \Rightarrow)$ the g-grammatical family of $G$. We also obtain the notions of $g$-form equivalence and strong $g$-form equivalence analogously. By the definition of $g$ - and s-interpretations it follows that $\xi_{s}(G) \subseteq \xi_{g}(G)$ and $\mathcal{Z}_{\mathrm{S}}(G, \Rightarrow) \subseteq \mathcal{Z}_{\mathrm{g}}(\mathrm{G}, \Rightarrow)$.

Returning to our previous examples we have already demonstrated that for:

$$
\begin{aligned}
& G_{1}: S \rightarrow \text { we have } \mathcal{L}_{g}\left(G_{1}, \Rightarrow\right) \geq \mathcal{L}(\text { FIN }) \\
& \text { and } \mathcal{L}_{\mathrm{s}}\left(\mathrm{G}_{1}, \Rightarrow \neq \mathcal{L}(\mathrm{FIN})\right. \text {. } \\
& G_{2}: S+S S ; S \rightarrow \text { a we have } \mathcal{L}_{\mathrm{g}}\left(G_{2}, \Rightarrow\right) \equiv \mathcal{L}_{\mathrm{s}}\left(G_{2}, \Rightarrow\right) \supseteq \mathcal{L}(C F) \\
& \text { and hence equality holds in both cases, since } \\
& \text { we can never obtain more than the context-free } \\
& \text { languages from a grammar form, } \\
& G_{3}: S \rightarrow a ; S \rightarrow \text { aS; } S \rightarrow \text { aSS again we have } \mathcal{L}_{S}\left(G_{3} \Rightarrow\right)=\mathcal{L}_{g}\left(G_{3}, \Rightarrow\right) \\
& =\not{\alpha}(\mathrm{CF}) \text {. } \\
& G_{4}: S \rightarrow a^{i}, 1 \leq i \leq 13, s \rightarrow a^{3} S a^{2} S a^{7} \text { once more we have } \\
& \mathcal{L}_{\mathrm{s}}\left(\mathrm{G}_{4}, \Rightarrow\right)=\mathcal{L}_{\mathrm{g}}\left(\mathrm{G}_{4}, \Rightarrow\right)=\mathcal{L}(\mathrm{CF}) \text {. }
\end{aligned}
$$

Note that we can derive $\mathcal{L}_{\mathrm{g}}\left(G_{3}, \Rightarrow\right)=\mathcal{L}_{\mathrm{g}}\left(G_{4}, \Rightarrow\right)=\mathcal{L}$ (CF) from the fact 


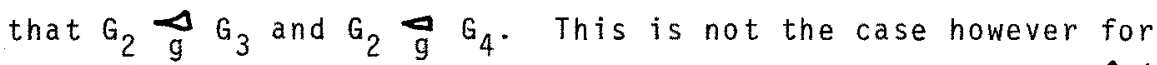
s-interpretation. In the next section we will show that $\mathcal{L}_{\mathrm{g}}\left(G_{1}, \Rightarrow\right)$ $=\mathcal{L}(\mathrm{FIN})$ and moreover that there is no grammar form $G$ with $\mathcal{L}_{S}(G, \Rightarrow)=\mathscr{L}(F I N)$. In fact this is the only g-grammatical family which is not s-grammatical as will be shown in Section 4.4 .

We close the present section by characterizing g-interpretations in terms of s-interpretations and closed terminal grammar morphisms. First observe however that whenever $G_{2} \underset{s}{\nabla} G_{1}(\mu)$, for two grammar forms $G_{i}=\left(V_{i}, \Sigma_{i}, P_{i}, S_{i}\right), i=1,2$, then an inverse of $\mu, \mu^{-1}$ can be defined and this is a very fine grammar morphism (see section I.2.4). Define $\mu^{-1}$ by, for a $11 x_{2}$ in $V_{2}, \mu^{-1}\left(x_{2}\right)=x_{1}$ in $V_{1}$ when $x_{2}$ is in $\mu\left(x_{1}\right)$ and $\mu^{-1}\left(x_{2}\right)=\emptyset$ otherwise. Since $\mu$ is a dfl-substitution on $v_{1}$ it follows from the definition of s-interpretation that $\mu^{-1}$ is a very fine grammar morphism. Define $\mu^{-1}$ by, for all $x_{2}$ in $v_{2}, \mu^{-1}\left(x_{2}\right)=x_{1}$ in $V_{1}$ when $x_{2}$ is in $\left(x_{1}\right)$ and $\mu^{-1}\left(x_{2}\right)=0$ otherwise. Since $\mu$ is a dfl-substitution on $V_{1}$ it follows from the definition of s-interpretation that $\mu^{-1}$ is a very fine grammar morphism. The converse holds, namely, whenever $\mu: G_{2} \rightarrow G_{1}$ is a very fine grammar morphism then $\mu^{-1}$ is a dfisubstitution and $G_{2} \underset{s}{\Delta} G_{1}\left(\mu^{-1}\right)$.

Theorem 1.1

Let $G_{i}=\left(V_{i}, \Sigma_{i}, P_{i}, S_{i}\right), i=1,2$ be two grammar forms.

(1) $G_{2} \underset{s}{\Delta} G_{1}(\mu)$ iff $\mu^{-1}: G_{2} \rightarrow G_{1}$ is a very fine grammar morphism.

(2) $G_{2} \rightarrow G_{1}(\mu)$ iff there exists some $G_{0}=\left(V_{0}, \Sigma_{0}, P_{0}, S_{0}\right)$ such that $G_{0} \underset{s}{a} G_{1}(\bar{\mu})$ and a closed terminal grammar morphism $\phi: G_{0} \rightarrow G_{2}$.

Proof: (1) clear by the remarks above.

(2) if: Since $\phi$ does not affect the nonterminals of $G_{0}$, we must have $V_{0}-\Sigma_{0}=V_{2}-\Sigma_{2}$.

Define $\mu$ on $V_{1}$ by:

$\mu(A)=\bar{\mu}(A)$, for all $A$ in $V_{1}-\Sigma_{1}$, and

$\mu(\mathrm{a})=\phi(\bar{\mu}(\mathrm{a}))$, for all a in $\Sigma_{1}$.

Let $P_{2}=\phi\left(P_{0}\right)$ and $S_{2}=S_{0}$. Clearly $G_{2} \underset{g}{Q} G_{1}(\mu)$

only if: Construct $G_{0}$ as follows:

$v_{0}-\Sigma_{0}=v_{2}-\Sigma_{2}, \Sigma_{0}=\left\{[x]: x\right.$ is in $\mu(a)$, for some a in $\left.\Sigma_{1}\right\}$, where the $[x]$ are new symbols. Define a homomorphism $\phi: V_{0}^{*} \rightarrow V_{2}^{*}$ by $\phi([x])=x$, for all $[x]$ in $\Sigma_{0}$. and $\phi(A)=A$, for all $A$ in $V_{0}-\Sigma_{0}$. Define an s-interpretation $\bar{\mu}$ of $G_{1}$ by:

$\bar{\mu}(A)=\mu(A)$, for all $A$ in $V_{1}-\Sigma_{1}$, 
and $\bar{\mu}(a)=[[x]: x$ is in $\mu(a)\}$, for a 11 a in $\Sigma_{1}$.

Let $P_{0}=\left\{A \rightarrow \alpha: A \rightarrow \gamma\right.$ is in $\mu(B \rightarrow B)$ for some $B \rightarrow \beta$ in $P_{1}$,

$A \rightarrow \alpha$ is in $\bar{\mu}(B \rightarrow \beta)$ and $\phi(\alpha)=\gamma\}$

and $S_{0}=S_{2}$.

Immediately $\phi: G_{0} \rightarrow G_{2}$ is a closed terminal grammar morphism, hence the result.

Since a closed terminal grammar morphism $\phi: G_{0} \rightarrow G_{2}$ has the property that $L\left(G_{2}, \Rightarrow\right)=\left(L\left(G_{0}, \Rightarrow\right)\right)$ we immediately have the following result:

\section{Coroliaxy 1.2}

Let $G$ be a grammar form, then $\mathcal{L}_{g}(G, \Rightarrow)=\mathcal{H}\left(\mathcal{L}_{s}(G, \Rightarrow)\right)$, where I denotes the homomorphic closure operator. For two grammar forms

$G_{1}$ and $G_{2}$ such that $\mathcal{Z}_{s}\left(G_{1}, \Rightarrow\right)=\mathcal{L}_{s}\left(G_{2}, \Rightarrow\right)$, we have $\mathcal{L}_{g}\left(G_{1}, \Rightarrow\right)=\mathcal{L}_{g}\left(G_{2}, \Rightarrow\right)$, in other words $s$-form equivalence implies $g-$ form equivalence. Similarly if $\mathcal{L}_{\mathrm{s}}\left(G_{1}, \Rightarrow\right) \subseteq \mathcal{Z}_{\mathrm{s}}\left(G_{2}, \Rightarrow\right)$ then $\mathcal{L}_{\mathrm{g}}\left(G_{1}, \Rightarrow\right) \subseteq \mathcal{L}_{\mathrm{g}}\left(G_{2}, \Rightarrow\right)$. 


\section{II.2 Isolation and Simulation}

We devote this section to two technical notions which have however far-reaching consequences. We demonstrate some of these consequences by characterizing when a grammar form gives exactly. the families of finite, regular and context-free languages under $g$ interpretations and characterizing the g-grammatical families of twosymbol forms, that is grammar forms which have only a single terminal symbol and only a single nonterminal symbol.

First observe the close relationship between the derivations in a g-or s-interpretation grammar and its form grammar.

\section{Lremma 2.1}

Let $G_{i}=\left(V_{i}, \Sigma_{i}, P_{i}, S_{i}\right), i=1,2$ be two grammars such that $G_{2} \underset{s}{\rightarrow} G_{1}(\mu)$ (or $\left.G_{2} \underset{g}{\Delta} G_{1}(\mu)\right)$. Then for every derivation $\alpha_{0}^{\prime} \Rightarrow \alpha_{1}^{\prime} \Rightarrow \ldots \Rightarrow \alpha_{m}^{\prime}$ in $G_{2}$, for some $\alpha_{i}^{\prime}$ in $V_{2}^{*}, 0 \leq i \leq m$ and $m>0$ there is a derivation

$\alpha_{0} \Rightarrow \alpha_{1} \Rightarrow \ldots \Rightarrow \alpha_{m}$ in $G_{1}$, for some $\alpha_{i}$ in $V_{1}^{*}$, such that $\alpha_{i}^{\prime}$ is in $\mu\left(\alpha_{i}\right), 0 \leq i \leq m$. When $G_{2} \underset{s}{ } G_{i}(\mu)$, then $\alpha_{i}=\mu^{-1}\left(\alpha_{i}^{\prime}\right)$, $0 \leq i \leq m$.

Proof: Clear.

Hence a derivation in an interpretation grammar is always an image of a derivation in the corresponding form grammar.

The statement of Lemma 2.1 can also be expressed by saying that for each derivation tree $\tau_{2}$ in $G_{2}$ there is a derivation tree $\tau_{1}$ in $G_{1}$ such that $\tau_{2}$ is in $\mu\left(\tau_{1}\right)$. In the case of s-interpretation there is exactiy one derivation tree $\tau_{1}$ in $G_{1}$ corresponding to each $\tau_{2}$ in $G_{2}$. Clearly $\tau_{1}$ and $\tau_{2}$ are obtained from each other by a relabelling of their nodes. Recall that two derivation trees $\tau_{1}$ and $\tau_{2}$ are equally shaped if they are obtained from each other by a relabelling of their non-frontier nodes.

\section{Coroltary 2.2}

Let $G_{i}=\left(V_{i}, \Sigma_{i}, P_{i}, S_{i}\right), i=1,2,3$ be three grammars such that $G_{1} \underset{s}{A} G_{3}\left(\mu_{1}\right)$ and $G_{2} \underset{s}{s} G_{3}\left(\mu_{2}\right)$. Suppose $\tau_{1}$ and $\tau_{2}$ are derivation trees in $G_{1}$ and $G_{2}$, respectively, of a word $\alpha$ in $V_{1}^{*} \cap V_{2}^{*}$ and there exists a derivation tree $\tau$ in $G_{3}$ such that $\tau_{1}$ is in $\mu_{1}(\tau)$ and $\tau_{2}$ is in $\mu_{2}(\tau)$. Then $\tau_{1}$ and $\tau_{2}$ are equally shaped. 
Proof: Since $\tau_{1}$ is in $\mu(\tau), \tau_{1}$ and $\tau$ are obtained from each other by relabelling of the nodes. Similarily $\tau_{2}$ and $\tau$ are obtained from each other by a relabelling of the nodes. Hence $\tau_{1}$ and $\tau_{2}$ are obtained from each other by a relabelling of the nodes. However by assumption $\tau_{1}$ and $\tau_{2}$ have the same frontier therefore $\tau_{1}$ and $\tau_{2}$ are equally shaped.

\section{Corozzary 2.3}

Let $G_{1}$ and $G_{2}$ be grammars such that either $G_{2} \vec{s}_{G_{1}}(\mu)$ or $G_{2} \Delta G_{1}(\mu)$. Then if $L\left(G_{2}, \Rightarrow\right)$ is infinite then $L\left(G_{1}, \Rightarrow\right)$ is infinite, but not conversely. This implies that if $L\left(G_{1}, \Rightarrow\right)$ is finite then $L\left(G_{2}, \Rightarrow\right)$ is finite, but not conversely.

Proof: If $L\left(G_{1}, \Rightarrow\right)$ is finite then $G_{1}$ has a finite number of non-empty sentential derivations. Since $\mu$ is finite $G_{2}$ also has a finite number of non-empty sentential derivations. Hence $L\left(G_{2} \Rightarrow\right)$ is finite.

This also gives a result promised in the previous section, namely:

\section{Coroltary 2.1}

Let $G: S \rightarrow a$, then $\mathcal{L}_{\mathrm{g}}(\mathrm{G}, \Rightarrow)=\mathcal{L}(\mathrm{FIN})$.

Proof: We have already demonstrated that $\mathscr{\alpha}_{\mathrm{g}}(G, \Rightarrow) \geq \mathcal{L}($ FIN) by Example 1.1. Equality holds by the previous corollary.

We now apply Lemma 2.1 in a less trivial manner to obtain another result promised in the previous section.

\section{Theorem 2.5}

Let $G$ be an arbitrary grammar form. Then $\mathcal{L}_{s}(G, \Rightarrow) \neq \mathcal{L}($ FIN $)$.

Proof: Assume $G$ is a grammar form for which $\mathcal{L}_{s}(G, \Rightarrow)=\mathcal{L}$ (FIN). Then $L(G, \Rightarrow)$ is finite, otherwise $\mathcal{\chi}_{s}(G, \Rightarrow) \notin(F I N)$. Choose an integer $m>\max (L S(G, \Rightarrow))$. We claim $L=\left\{a^{m}\right\}$ is not in $\mathcal{L}_{s}(G, \Rightarrow)$. If it is, then there exists $G^{\prime} \rightarrow G(\mu)$ with $L=L\left(G^{\prime} \Rightarrow\right)$, that is $S^{\prime} \Rightarrow^{+} a^{m}$ in $G^{\prime}$. Now by Lemma 2.7 this implies $\mu^{-1}\left(S^{\prime}\right) \Rightarrow^{+} \mu^{-1}\left(a^{m}\right)$ in $G$, giving a contradiction. Therefore $L$ is not in $\mathcal{L}_{s}(G, \Rightarrow)$ contradicting the assumption that $\mathcal{L}_{\mathrm{s}}(G, \Rightarrow)=\mathcal{L}($ FIN $)$. 
We now turn to our first technical result and "tool".

\section{II.2.1. Isolation}

Consider a grammar form $G=(V, \Sigma, P, S)$ and terminating derivations $A \Rightarrow^{+} x_{i}$ in $\Sigma^{*}, 1 \leq i \leq n$, for some $n \geq 1$ and some $A$ in $V-\Sigma$. Derive an interpretation grammar $G^{\prime} \underset{S}{\Delta} G$ and hence $G^{\prime} \underset{g}{G} G$, such that (i) $G^{\prime}=\left(V^{\prime}, \Sigma, P^{\prime}, S\right)$ with $V \subseteq V^{\prime},(i i)$ all productions in $P$ whose left hand side is not $A$ are taken into $P^{\prime}$ unchanged, and ( $i i i$ ) the remaining productions in $P^{\prime}$ only serve to derive the $x_{i}$ from $A$ and nothing else. Then we say that the derivations $A \Rightarrow^{+} x_{i}, 1 \leq i \leq n$ in $G$ have been isolated in $G^{\prime}$. In the following we formalize this notion for a derivation which is not necessarily terminating.

Let $G=(V, \Sigma, P, S)$ be a grammar form and $A \Rightarrow^{+} \alpha_{i}, 1 \leq i \leq n$, for some $n \geq 1$, be mutually distinct derivations in $G$, where $A$ is in $V-\Sigma$ and the $\alpha_{i}$ are in $V^{*}$. Recall that two derivations $A^{+} \alpha$ and $A \Rightarrow{ }^{+} B$ in a grammar $G$ are distinct if for the corresponding derivation trees neither one is a tree-prefix of the other. We construct a grammar form $G^{\prime} \underset{\$}{\Delta} G$ such that whenever $A \Rightarrow^{+} \beta$ in $G^{\prime}$ with $\beta$ in $V^{*}$, then the derivation can be re-arranged as $A \Rightarrow^{+} \alpha_{i} \Rightarrow^{*} \beta$, for some $i$, $1 \leq i \leq n$. In other words the derivations $A \Rightarrow{ }^{+} \alpha_{j}$ in $G$ become the "only possible" derivations for $A$ in $G$ ". Hence the $A \Rightarrow{ }^{+} \alpha_{i}$ have been isolated in $G^{\prime}$. Consider the case when $n=1$, the case for $n>1$ we 1 eave to the reader.

Let $A \Rightarrow^{+} \alpha$ be written

(*) $A=A_{0} \Rightarrow \alpha_{1} \Rightarrow \ldots \Rightarrow \alpha_{n}=\alpha$ for some $n>0$, where the production $A_{i} \rightarrow \beta_{i+1}$ is used at the $i$ th step, $0 \leq i<n$. Rewrite $\beta_{i}$ as $\gamma_{i, 1} A_{i, 1} \cdots A_{i, n_{i}} \gamma_{i, n_{i}+1}$ where $n_{i}=0$ implies $\beta_{i}=\gamma_{i, 1}, 1 \leq i \leq n$. In the case $n_{j} \geq 1$ then each $A_{j, j}$, is rewritten in the derivation $(*)$, while $\gamma_{j, j}$ contains no nonterminal which is rewritten in (*). Let $V(A)=\left\{\left[A_{i, j}, i, j\right]: A_{i, j}\right.$ in $\left.V-\Sigma, 1 \leq i \leq n, 1 \leq j \leq n_{j}\right\}$, and $P(A)=\left\{A \rightarrow \gamma_{1,1}\left[A_{1,1}, 1,1\right] \ldots\left[A_{1, n_{1}}, 1, n_{1}\right] \gamma_{1, n_{1}+1}\right\}$ $u\left\{[B, j, k] \rightarrow \gamma_{i, 1}\left[A_{i, 1}, i, 1\right] \ldots\left[A_{i, n_{j}}, i, n_{i}\right] \gamma_{i, n_{i}+1}:\right.$ where $B$ is the nonterminal rewritten at the $(i-1)$ st step in $(*)$ and $B=A_{j, k}$, that is $B$ is introduced at the $j$ th step, $j \leq \mathfrak{i}-1\}$.

Let $G^{\prime}=(V \cup V(A), \Sigma,(P-\{A \rightarrow \delta: A \rightarrow \delta$ in $P\}) \cup P(A), S)$. Observe that $G^{\prime}$ has indeed isolated the derivation $A \Rightarrow^{+} \alpha$ in $G$ by a suitable renaming of the nonterminals. Moreover these new nonterminals in $V(A)$ each have a single production and these can only be applied within 
the derivation specified by $A \Rightarrow^{+} \alpha$.

Now define $\mu$ by:

$\mu(a)=a$, for all a in $\Sigma$,

$\mu(A)=\{A\} \cup\{[A, i, j]: 1 \leq i<n, 1 \leq j \leq m\}$,

where $m=\max \left(\left\{n_{j}: 1 \leq i<n\right\}\right)$.

It should be clear that $P \cup P(A) \subseteq \mu(P)$ and hence $G^{\prime} \rightarrow G(\mu)$ and $G^{\prime} \rightarrow G(\mu)$.

This technique can be generalized to give:

\section{Lemma 2.6}

Let $G=(V, \Sigma, P, S)$ be a grammar form, $A$ be in $V-\Sigma$ and $A \Rightarrow^{+} \alpha_{i}$ be mutualiy distinct derivations in $G$, where $1 \leq i \leq n$, for some $n \geq 1$. Then there exists a grammar form $G^{\prime}=\left(V^{\prime}, \Sigma^{\prime}, P^{\prime}, S^{\prime}\right)$ such that

(i) $V \subseteq V^{\prime}, \Sigma=\Sigma^{\prime}, P-\{A \rightarrow \delta: A \rightarrow \delta$ in $P\} \subseteq P^{\prime}$ and $S=S^{\prime}$,

(ii) G' $\rightarrow$ G (and $G^{\prime} \underset{g}{\rightarrow} G$ ), and

(iii) whenever $A \Rightarrow^{+} \beta$ in $G^{\prime}$ with $B$ in $V *$ there is a derivation $A \Rightarrow \alpha_{i} \Rightarrow^{*} \beta$ in $G^{\prime}$, for some $i, 1 \leq i \leq n$.

We now demonstrate an application of isolation.

\section{Lemma 2.?}

Let $G=(V, \Sigma, P, S)$ be a grammar form. If $G$ is nonempty then $\mathcal{L}_{g}(G, \Rightarrow) \geq \mathcal{L}(F I N)$.

Proof: Since $G$ is nonempty there is a nonempty word $x$ in $\Sigma *$ such that $S \Rightarrow^{+} \mathrm{x}$. Consider an interpretation $G^{\prime}=\left(V^{\prime}, \Sigma^{\prime}, P^{\prime}, S\right){ }_{S} G$ which isolates this derivation. Then $L\left(G^{\prime} \Rightarrow\right)=\{x\}$. Second, consider an interpretation $G^{\prime \prime} \underset{g}{g} G^{\prime}(\mu), G^{\prime \prime}=\left(V^{\prime \prime}, \Sigma ", P^{\prime \prime}, S\right)$, where $\mu^{\prime}(A)=A$, for a $11 \mathrm{~A}$ in $V^{\prime}-\Sigma^{\prime}$ and $\mu^{\prime}(a)=\{a, \lambda\}$ for a 1$]$ a in $\Sigma$, and $P^{\prime \prime}$ is chosen such that $S \Rightarrow+a$ in $G^{\prime \prime}$, for some a in $\Sigma$ is the only derivation in $G^{\prime \prime}$. By Corollary 2.4 slightly modified $\mathcal{L}_{g}\left(G^{\prime \prime}, \Rightarrow\right)=\alpha(F I N)$. Hence

$$
\mathcal{L}(F I N)=\mathcal{L}_{g}\left(G^{\prime \prime}, \Rightarrow\right) \subseteq \mathcal{L}_{g}\left(G^{\prime}, \Rightarrow\right) \subseteq \mathcal{L}_{g}(G, \Rightarrow) \text { giving the result. }
$$

This result no longer holds under s-interpretation, for example consider $G$ defined by the productions:

$$
S \rightarrow \text { aas; } S \rightarrow \text { aa; }
$$

then $\{a\}$ is not in $\mathcal{L}_{S}(G, \Rightarrow)$ al though $G$ is nonempty.

We now characterize when a grammar form generates exactly the finite sets under g-interpretation. 
Theorem 2.8

Let $G=(V, \Sigma, P, S)$ be a nonempty grammar form. Then $\mathcal{L}_{g}(G, \Rightarrow)=\mathcal{L}(F I N)$ iff $G$ is finite.

Proof: if: Since $G$ is finite, $\mathcal{L}_{\mathrm{g}}(G, \Rightarrow) \subseteq \mathcal{Z}($ FIN) by Corollary 2.3 . Equality follows by Lemma 2.7 .

only if: Since $L(G, \Rightarrow)$ is in $\mathcal{L}($ FIN), $G$ is finite.

Thus Theorem 2.5 and 2.8 provide a contrast of the effects of $s$ - and g-interpretation with respect to the finite sets. As will be proved in Section 4.3 the only g-grammatical family which is not $s$-grammatical is $\mathcal{L}$ (FIN).

We can consider a weaker requirement, namely when is $\mathcal{L}$ (FIN) contained in an s-grammatical family. As we shall see in section 4.3 whenever $\mathcal{L}$ (FIN) is contained in an s-grammatical family $\mathcal{L}$, then $\mathcal{Z}$ (REG) is also contained in $\mathcal{L}$. Moreover such a containment can be characterized in terms of a simple condition on the corresponding grammar form.

\section{II.2.2 Simulation}

The second important technique can be described as follows. Let $G_{1}$ and $G_{2}$ be grammar forms such that $A \Rightarrow^{+} \alpha$ in $G_{2}$ for each production $A \rightarrow \alpha$ in $G_{1}$. We say $\underline{G}_{2}$ simulates $G_{1}$ or $\underline{G}_{2}$ production-simulates $G_{1}$. In this case $\mathcal{L}_{s}\left(G_{1}, \Rightarrow\right) \subseteq \mathcal{L}_{s}\left(G_{2}, \Rightarrow\right)$.

Lemma 2.9

Let $G_{i}=\left(V_{i}, \Sigma_{i}, P_{i}, S\right), i=1,2$ be two grammar forms such that for each production $A \rightarrow \alpha$ in $P_{1}$ there is a derivation $A \Rightarrow^{+} \alpha$ in $G_{2}$ and $\Sigma_{1} \subseteq \Sigma_{2}$. Then $\mathcal{L}_{\mathrm{S}}\left(G_{1}, \Rightarrow\right) \subseteq \mathcal{L}_{\mathrm{S}}\left(G_{2}, \Rightarrow\right)$.

Proof: We construct a grammar form $G=\left(V_{2}, \Sigma_{2}, P, S\right)$ such that $\mathcal{Z}_{\mathrm{s}}\left(G_{1} \Rightarrow\right) \subseteq \mathcal{L}_{\mathrm{s}}(G, \Rightarrow)$ and $\mathcal{Z}_{\mathrm{s}}(G, \Rightarrow)=\mathcal{Z}_{\mathrm{s}}\left(G_{2}, \Rightarrow\right)$.

Let $P=P_{1} \cup P_{2}$. Clearly $\mathcal{L}_{s}\left(G_{1}, \Rightarrow\right) \subseteq \mathcal{L}_{s}(G, \Rightarrow)$ since $G_{1}$ is a subgrammar of $G$, and $\mathcal{L}_{\mathrm{s}}\left(G_{2}, \Rightarrow\right) \subseteq \mathcal{L}_{\mathrm{s}}(G, \Rightarrow)$ by the same reasoning.

It remains to show that $Z_{s}(G, \Rightarrow) \subseteq \mathcal{L}_{S}\left(G_{2}, \Rightarrow\right)$. Consider a grammar $G^{\prime} \rightarrow G(\mu)$. It suffices to show that $L\left(G^{\prime}, \Rightarrow\right)$ is in $\mathcal{L}_{S}\left(G_{2}, \Rightarrow\right)$. Letting $G^{\prime}=\left(V_{2}^{\prime}, \Sigma_{2}^{\prime}, P^{\prime}, S^{\prime}\right)$ it is clear that we can partition $P^{\prime}$ into $P_{1}^{\prime} \cup P_{2}^{\prime}$, that is those productions which are interpretations of $G_{1}$-productions and those of $G_{2}$-productions. For all productions 
$A^{\prime} \rightarrow \alpha^{\prime}$ in $P_{j}^{\prime}, A^{\prime} \rightarrow \alpha^{\prime}$ is in $\mu(A \rightarrow \alpha)$ for some $A \rightarrow \alpha$ in $P_{1}$, hence there is a derivation $A \Rightarrow^{+} \alpha$ in $G_{2}$. Using the technique of Lemma 2.6 construct unique isolation derivations $A^{\prime} \Rightarrow^{+} \alpha^{\prime}$ from $A \Rightarrow^{+} \alpha$ in $G_{2}$. In other words construct $G_{2}^{\prime} \Delta_{S} G_{2}$, where $G_{2}^{\prime}=\left(V_{2}^{\prime \prime}, \Sigma_{2}^{\prime}, P_{2}^{\prime \prime}, S^{\prime}\right)$ with $V_{2}^{\prime \prime}$ equal to $V_{2}^{\prime}$ together with all new symbols introduced by the isolating derivations and $P_{2}^{\prime \prime}$ equal to $P_{2}^{1}$ together with all the necessary new productions of the isolating derivations. Now immediately we have:

$A^{\prime} \Rightarrow^{+} \alpha^{\prime}$ in $G_{2}^{\prime}$ for each production $A^{\prime} \rightarrow \alpha^{\prime}$ in $G^{\prime}$, and furthermore $L\left(G_{2}^{\prime} \Rightarrow\right)=L\left(G^{\prime} \Rightarrow\right)$ since the new productions in $P_{2}^{\prime \prime}$ can only be applied in such derivations. As $G_{2}^{\prime}$ is an s-interpretations of $G_{2}$ we obtain the result.

One immediate consequence is the following useful corollary.

\section{Corotzary 2.10}

Let $G=(V, \Sigma, P, S)$ be a grammar form and let $A \Rightarrow^{*} \alpha$ be in $G$ for some $A$ in $V-\Sigma$ and $\alpha$ in $V^{*}$. Then $F=(V, \Sigma, P \cup\{A \rightarrow \alpha\}, S)$ is $s-$ form equivalent to $G$ (and hence they are $g$-form equivalent).

Proof: $\mathscr{L}_{S}(G, \Rightarrow) \subseteq \mathscr{L}_{s}(F, \Rightarrow)$ is immediate. On the one hand, if $A \Rightarrow+\alpha$ in $G$ then Lemma 2.9 yields the result. On the other hand, if $A \Rightarrow A$ in $G$, it is clear that adding the production $A \rightarrow A$ to $G$ does not affect $\mathcal{L}_{\mathrm{S}}(G, \Rightarrow)$.

As an application of the above corollary we have:

\section{Lemma 2.11}

Let $G=(V, \Sigma, P, S)$ be a reduced grammar form. Let $G_{A}=(V, \Sigma, P, A)$ for some $A$ in $V-\Sigma$. Then $\mathcal{L}_{g}\left(G_{A}, \Rightarrow\right) \subseteq \mathcal{L}_{g}(G, \Rightarrow)$.

Proof: Since $G$ is reduced there exists a derivation

$S \Rightarrow x A y \Rightarrow^{+} x y z$ in $\Sigma^{*}$.

By a straightforward extension of Corollary 2.10 not only can we add $S \rightarrow x A y$ to $P$ to give a $g$-form equivalent grammar form, but also we can add $S \rightarrow A$ to $P$ to give a $g$-form equivalent grammar form. Hence assume $P$ contains $S \rightarrow A$. We construct $G^{\prime}=\left(V \cup\{Z\}, \Sigma, P^{\prime}, Z\right)$ such that $G^{\prime} \overleftrightarrow{g}_{G(\mu)}$ and $\mathcal{L}_{g}\left(G^{\prime}, \Rightarrow\right)=\mathcal{L}_{g}\left(G_{A}, \Rightarrow\right)$, where $\mu$ is defined by:

for a $11 X$ in $V-\{S\}, \mu(X)=X$ and $\mu(S)=\{S, Z\}$.

Define $P^{\prime}$ by:

$P^{\prime}=P \cup\{Z+A\}$. Clearly $G^{\prime} \vec{g} G(\mu)$ and $L\left(G_{A} \Rightarrow\right)=L\left(G^{\prime}, \Rightarrow\right)$

and further $\mathcal{L}_{g}\left(G_{A}, \Rightarrow\right)=\mathcal{L}_{g}\left(G^{\prime}, \Rightarrow\right) \subseteq \mathcal{L}_{g}\left(G_{g} \Rightarrow\right)$. 
Second, we apply Corollary 2.10 to obtain the following "backsubstitution" result, a technical result which is useful in section 4.2 .

\section{Lemma 2.12-- The Back-Substitution Lemma}

Let $G=(V, \Sigma, P, S)$ be a grammar form and $A$ in $V-\{S\}$ be an arbitrary nonterminal with productions

$A \rightarrow \alpha_{1}|\ldots| \alpha_{r}$ in $P$.

Construct $\bar{G}=(V, \Sigma, \bar{P}, S)$ from $G$ as follows: Replace each production $B \rightarrow B$ in $P$ with all possible productions obtained by replacing each $A$ in $\beta$ with $\alpha_{1}, \ldots, \alpha_{r}$. The resulting set of productions is denoted by $\bar{P}$. Then $G$ and $\bar{G}$ are $s-$ and $g$-form equivalent.

Proof: $\quad \mathcal{L}_{\mathrm{S}}(\overline{\mathrm{G}}, \Rightarrow) \subseteq \underset{+}{\mathrm{S}}(\mathrm{G}, \Rightarrow)$ since for each production $D \rightarrow \gamma$ in $\bar{G}$ there is a derivation $D \Rightarrow^{+} \gamma$ in $G$. Conversely consider $\mathcal{L}_{S}(G, \Rightarrow) \subseteq \mathcal{L}_{S}(\bar{G}, \Rightarrow)$. Let $G^{\prime}=\left(V^{\prime}, \Sigma^{\prime}, P^{\prime}, S^{\prime}\right)$ be an arbitrary s-interpretation of $G, G^{\prime} \Delta \vec{S}(\mu)$, then it is straightforward to construct a $\bar{G}^{\prime} \underset{S}{G}$ by carrying out the construction of the lemma for $G^{\prime}$ rather than $G$ and for all $A^{\prime}$ in $\mu(A)$ rather than for $A$ only. The resulting $\bar{G}^{\prime}$ is an s-interpretation of $\bar{G}$ and by standard results $L\left(\bar{G}^{\prime}, \Rightarrow\right)=L\left(G^{\prime}, \Rightarrow\right)$.

Third, we apply corollary 2.10 to characterize when a grammar form gives exactly the regular languages and the context-free languages under g-interpretation. In the latter case we say such a form $G$ is g-complete, i.e. $\mathcal{L}_{\mathrm{g}}(G, \Rightarrow)=\mathcal{L}(\mathrm{CF})$.

\section{Lemma 2.13}

Let $G=(V, \Sigma, P, S)$ be an infinite grammar form. Then $\mathcal{L}_{\mathrm{g}}(G, \Rightarrow) \geq \mathcal{L}(\mathrm{REG})$.

Proof: Since $G$ is infinite there exists a nonterminal $A$ in $V$ such that

$$
S \Rightarrow+u A v \Rightarrow^{+} \text {uwAyv } \Rightarrow^{+} \text {uwxyv with wy } \neq \lambda \text { and } x \neq \lambda
$$

otherwise $L(G, \Rightarrow)$ would be finite.

Construct $G_{1}=(V, \Sigma, P \cup\{A \rightarrow x, A \rightarrow W A y\}, S)$ then by corollary 2.10 $G_{1}$ is $g$-form equivalent to $G$. Consider $G_{1} \underset{g}{G} G_{1}$ defined by $G_{1}^{\prime}=\left(V, \Sigma, P_{j}^{\prime}, S\right)$, where $P_{j}^{\prime}$ consists of $P, A \rightarrow$ a and either $A \rightarrow$ a $A$ if $w \neq \lambda$ or $A \rightarrow A a$ if $w=\lambda$. Without loss of generality assume $P_{j}$ includes $A \rightarrow$ aA. 
Now define a new grammar $F$ jy $F=\left(V, \Sigma, P_{j}^{\prime}, A\right)$ then, by Lemma $2.11, \mathscr{Z}_{\mathrm{g}}(F, \Rightarrow) \subseteq \mathcal{L}_{\mathrm{g}}\left(G_{1}^{\prime}, \Rightarrow\right)$. Finally consider $F^{\prime}=(\{A, a\},\{a\},\{A \rightarrow a ; A \rightarrow a A\}, A) . \quad C l e a r l y F^{\prime} \rightarrow \vec{g}$ and furthermore any right linear grammar is a g-interpretation of $F^{\prime}$, hence $\mathcal{L}(R E G) \subseteq \mathcal{L}_{g}\left(F^{\prime}, \Rightarrow\right) \subseteq \mathcal{Z}_{g}(F, \Rightarrow) \subseteq \mathcal{L}_{g}(G ; \Rightarrow) \subseteq \mathcal{Z}_{g}(G, \Rightarrow)$. Therefore
$\mathcal{L}_{g}(G, \Rightarrow) \geq \mathcal{L}(R E G)$.

We now turn to the characterization theorem.

\section{Theorem 2.14}

Let $G=(V, \Sigma, P, S)$ be an infinite reduced grammar form. Then $\mathcal{L}_{g}(G, \Rightarrow)=\mathcal{L}(R E G)$ iff $G$ is non-self-sembedding.

Proof: if: By the previous 1emma observe that $\mathcal{L}_{\mathrm{g}}(G, \Rightarrow) \geq \mathcal{L}(\mathrm{REG})$. To show the converse observe that each $G^{\prime} \vec{g} G$ is also non-selfembedding. [Assume otherwise, then because derivations in the interpretation grammar are images of derivations in the form grammar, $G^{\prime}$ is self-embedding implies $G$ is self-embedding, a contradiction.] Hence by a standard result in language theory, $L\left(G^{\prime}, \Rightarrow\right)$ is regular, hence $\mathcal{Z}_{g}(G, \Rightarrow)=\mathcal{L}(R E G)$.

only if: Assume $G$ is self-embedding. Since $G$ is reduced there exists a nonterminal $A$ such that

$S \Rightarrow$ * $u A v \Rightarrow+$ uwAyv $\Rightarrow^{+}$uwxyv where $w \neq \lambda$ and $y \neq \lambda$.

Without loss of generality, assume $P$ contains the productions $A \rightarrow a A b$ and $A \rightarrow \lambda$ by Corollary 2.10 and previous remarks. Now $G_{A}=(V, \Sigma, P, A)$ has $\mathcal{L}_{g}\left(G_{A} \Rightarrow\right) \subseteq \mathcal{L}_{g}(G, \Rightarrow)$. However

$G_{A}^{\prime}=(\{A, a, b\},\{a, b\},\{A \rightarrow a A b, A \rightarrow \lambda\}, A)$, generates the language $L\left(G_{A}^{\prime}\right)=\left\{a^{j} b^{i}: i \geq 0\right\}$ a well known non-regular language. This yields a contradiction.

Our next result gives necessary and sufficient conditions for a grammar form to be g-complete.

\section{Theorem 2.15}

Let $G=(V, \Sigma, P, S)$ be a reduced grammar form. Then $\mathcal{L}_{\mathrm{g}}(G, \Rightarrow)=\mathcal{L}(\mathrm{CF})$ iff $G$ is expansive. 
Proof: if: Since $G$ is expansive there is a nonterminal $A$ such that $A \Rightarrow{ }^{+} u_{1} A u_{2} A u_{3} \Rightarrow^{+} u_{1} x u_{2} x u_{3}, x \neq \lambda$, hence by Corollary 2.10 we can assume $A \rightarrow x$ and $A \rightarrow u_{1} A_{u_{2}} A u_{3}$ are in $P$ without any loss of generality. Consider $G_{A}$ and an interpretation $F \underset{g}{G} G_{A}$, where $F$ is defined by: $F=(\{A, a\},\{a\},\{A \rightarrow a, A \rightarrow A A\}, A)$,

clearly $Z_{\mathrm{g}}(\mathrm{F}, \Rightarrow)=\mathcal{Z}(\mathrm{CF})$, hence $\mathcal{Z}_{\mathrm{g}}(\mathrm{G}, \Rightarrow)=\mathcal{Z}(\mathrm{CF})$ by Lemma 2.11 . only if: Since $\mathscr{\chi}_{\mathrm{g}}(G, \Rightarrow)=\not{L}(\mathrm{CF})$ then there is an interpretation $G^{\prime}$ with $L\left(G^{\prime}, \Rightarrow\right)=D_{1}$, the Dyck set on one letter. It is well known that $G^{\prime}$ must be expansive hence $G$ is expansive.

We can obtain a similar characterization theorem for the linear languages.

\section{Theorem 2.16}

Let $G$ be a reduced grammar form. Then $\mathcal{L}_{g}(G, \Rightarrow)=\mathcal{L}(L I N)$ iff $G$ is self-embedding and if $S \Rightarrow{ }^{+} u A x B y$ then $A$ and $B$ are not both selfembedding.

Proof: Left to the reader.

It is worthwhile remarking that none of the above characterization results hold under s-interpretation, since Lemma 2.11 does not hold in this case.

We may summarize the results so far in:

\section{Theorem 2.17}

Given an arbitrary grammar form $G$ it is decidable whether or not $\mathcal{Z}_{\mathrm{g}}(\mathrm{G}, \Rightarrow)=\mathcal{L}(\mathrm{FIN}), \mathcal{L}(\mathrm{REG}), \mathcal{L}(\mathrm{LIN})$ or $\mathcal{L}(\mathrm{CF})$.

We now consider two-symbol forms under g-interpretations, that is forms which have a single terminal a and a single nonterminal S. Given a two-symbol form we can characterize its language family as being one of five possible language families.

\section{Theorem 2.18}

Let $G=(\{S, a\},\{a\}, P, S)$ be a two-symbol form, then $\mathcal{L}_{g}(G, \Rightarrow)$ is in $\{\{\emptyset\}, \mathcal{L}(\mathrm{FIN}), \mathcal{L}(\mathrm{REG}), \mathcal{L}(\mathrm{LIN}), \mathcal{L}(\mathrm{CF})\}$. 
Proof: Clearly if $L(G, \Rightarrow)=\emptyset$ or $\{\lambda\}$ then $\mathcal{L}_{\mathrm{g}}(G, \Rightarrow)=\{\emptyset\}$. If $G$ is finite then $\mathcal{L}_{\mathrm{g}}(G, \Rightarrow)=\mathcal{L}$ (FIN) by Theorem 2.8. If $G$ is infinite, clearly $G$ is reduced, therefore $\mathcal{L}_{g}(G, \Rightarrow)=\mathscr{L}(R E G)$ only if $G$ is nonself-embedding, that is all productions must have at most one $S$ on the right hand side and either all nonterminating productions are of type $S \rightarrow a^{i} S, i \geq 0$ or all nonterminating productions are of type $S \rightarrow S a^{i}, i \geq 0$. Similarly if $G$ is infinite and it contains a production of type $S \rightarrow \alpha S \beta S \gamma, \alpha \beta \gamma$ in $\{S, a\}^{*}$, then $\mathcal{L}_{g}(G, \Rightarrow)=\mathcal{L}(C F)$. otherwise $\chi_{g}(G, \Rightarrow)=\mathcal{L}(L I N)$.

Before leaving this simulation section we consider two other technical results which have a simulation flavor.

Lemma 2.19 -- Rep Lacement Lemma

Let $G=(V, \Sigma, P, S)$ be a grammar form and let $A$ in $V-\Sigma$ be an arbitrary nontermina 1. Let $E=(U, \Delta, Q, A)$ be a grammar form which is $s$-form equivalent to the subgrammar form $G_{A}$ of $G$, where $(U-\Delta) \cap(V-\Sigma)=\{A\}$.

Then the grammar form $H$ obtained by removing all A-productions from $P$ and adding all the productions in $Q$ is s-form equivalent to $G$.

Proof: Without loss of generality assume $G$ is reduced. If $A=S$, then $H=(U, \Sigma \Delta,(P-\{S \rightarrow \alpha: S \rightarrow \alpha$ in $P\}) \cup Q, S)$ is clearly $s$-form equivalent to $G$ as desired, since the productions in $H$ taken from $P$ are unreachable. Therefore we now assume $A \neq S$ from hereon in.

Let the reduced subgrammar form $G_{A}=\left(V_{A}, \Sigma_{A}, P_{A}, A\right)$ and denote by $G_{-A}$, the grammar form defined by:

$G_{-A}=\left(\left(V-V_{A}\right) \cup\{A\} \cup \Sigma, \Sigma, P-(\{A \rightarrow \alpha: A \rightarrow \alpha\right.$ is in $\left.P\}), S\right)$.

$G_{-A}$ is the "complement" subgrammar of $G$ with respect to $G_{A}$. Its importance stems from the following observation:

(*) Each derivation $S \Rightarrow^{*} x$ in $G$ can be uniquely rearranged as $S \Rightarrow^{*} \alpha \Rightarrow^{*} x$ in $G$, for some $\alpha$ in $V^{*}$, where $S \Rightarrow^{*} \alpha$ and $\alpha \Rightarrow^{*} x$ are derivations in $G_{-A}$ and $G_{A}$ respectively. Consider $H=(V \cup \cup, \Sigma \cup \Delta,(P-\{A \rightarrow \alpha: A \rightarrow \alpha$ is in $P\}) \cup Q, S)$. We only show that $\mathcal{L}_{S}(G, \Rightarrow) \subseteq \mathcal{L}_{S}(H, \Rightarrow)$, since the reverse inclusion follows by the same argument because $G$ can be written as $G=\left(V \cup V_{A}, \Sigma \cup \Sigma_{A},(P-\{A \rightarrow \alpha: A \rightarrow \alpha\right.$ is in $\left.P\}) \cup P_{A}, S\right)$. Consider an arbitrary $G^{\prime} \triangle_{S} G(\mu)$, where $G^{\prime}=\left(V^{\prime}, \Sigma^{\prime}, P^{\prime}, S^{\prime}\right)$ and $\mu(A)=\left\{A_{1}, \ldots, A_{n}\right\}$ say. For each $A_{i}$ denote the corresponding subgrammar by $G_{i}^{\prime}$. Now by assumption there is an $E_{i} \Delta \vec{s}$ such that 
$L\left(E_{i}, \Rightarrow\right)=L\left(G_{i}^{\prime} \Rightarrow\right)$, for all $i, 1 \leq i \leq n$. Let each $E_{i}=\left(U_{i}, \Delta_{i}, Q_{i}, A_{i}\right)$ be such that $\left(U_{i}-\Delta_{j}\right) \cap\left(V^{\prime}-\Sigma^{1}\right)=\left\{A_{j}\right\}, 1 \leq i \leq n$ and also assume that $\left(U_{j}-\Delta_{j}\right) \cap\left(U_{j}-\Delta_{j}\right)=\emptyset$ for all $i, j, 1 \leq i<j \leq n$.

Now construct an $H^{\prime}=\left(V^{\prime \prime}, \Sigma^{\prime \prime}, P^{\prime \prime}, S^{\prime}\right)$ as follows:

$$
\begin{aligned}
& v^{\prime \prime}=v^{\prime \prime} \cup \bigcup_{i=1}^{n} U_{i}, \Sigma^{\prime \prime}=\Sigma^{\prime} \cup \bigcup_{i=1}^{n} \Delta_{i} \text { and } \\
& p^{\prime \prime}=P^{\prime}-\left(\left\{A_{i} \rightarrow \alpha: A_{i} \rightarrow \alpha \text { is in } P^{\prime}, I \leq i \leq n\right\}\right) \cup \bigcup_{i=1}^{n} Q_{i} \text {. }
\end{aligned}
$$

It should be clear that $H^{\prime} \rightarrow H$ from the construction and moreover $L\left(H^{\prime} \Rightarrow\right)=L\left(G^{\prime}, \Rightarrow\right)$ by $(*)$ applied to the inverse images of the derivations in $G^{\prime}$ and $H^{\prime}$.

This completes the lemma.

Given a grammar form $G=(V, \Sigma, P, S)$ it is straightforward to prove that $G$ has an $s$-form equivalent grammar form $G^{\prime}=\left(V^{\prime}, \Sigma, P^{\prime}, S^{\prime}\right)$ satisfying (i) $V^{\prime}=V \cup\left\{S^{\prime}\right\}$, where $S^{\prime}$ is a new nonterminal, (ii) $G^{\prime} \underset{S}{\&} G$ and $(i i i) P^{\prime}$ is the same as $P$ except that for each production $S \rightarrow \alpha$ in $P$ we also take $S^{\prime} \rightarrow \alpha$ into $P^{\prime}$.

We now prove a similar but stronger result for a nonterminal which is not the sentence symbol.

\section{Lemma $2.20-(A, \alpha, B, \beta)$-Partition Lemma}

Let $G=(V, \Sigma, P, S)$ be a grammar form and let $A \rightarrow \alpha B \beta$ be an arbitrary production in $P$, where $B$ is a nonterminal and $\alpha \beta$ is a word over $V$. Then we may assume:

(i) this is the only appearance of $B$ on the right hand side of productions in $P$,

(ii) letting the reduced subgrammar form $G_{B}=\left(V_{B}, \Sigma_{B}, P_{B}, B\right)$ then $P-P_{B}$ has only the nonterminal $B$ in common with $V_{B}$, and

(iii) $\alpha \beta$ has no nonterminals in common with $V_{B}$.

Proof: Without loss of generality we may assume $G$ is reduced and by Lemma 2.19 we may also assume that the reduced grammar forms $G_{B}=\left(V_{B}, \Sigma_{B}, P_{B}, B\right)$ and $G_{-B}\left(V_{-B}, \Sigma_{-B}, P_{-B}, S\right)$, (the sub-grammar form and complement subgrammar form of $G$ with respect to $B$, respectively) have onty $B$ as their common nonterminal. Thus condition ( $i j$ ) is satisfied. Define $G_{B^{\prime}}=\left(V_{B^{\prime}}, \Sigma_{B}, P_{B^{\prime}}, B^{\prime}\right) \overrightarrow{f s} G_{B}(\mu)$, where $\mu(C)=C^{\prime}$ a new nonterminal, for all $C$ in $V_{B}-\Sigma_{B}, \mu(a)=$ a for all a in $\Sigma_{B}$ and $P_{B}=\mu\left(P_{B}\right)$. This renamed version of $G_{B}$ is chosen such that $\left(V_{B},-\Sigma_{B}\right) \cap(V-\Sigma)=\emptyset$. By a slight generalization of Lemma 2.19, if 
we replace any production $C \rightarrow \delta B_{\gamma}$ in $P$ by $C \rightarrow \delta B^{\prime} \gamma$ and add all the productions in $P_{B}$, then the resulting grammar is s-form equivalent to $G$.

Carry out this replacement of $B$ by $B$ " for all appearances of $B$ in the productions of $P_{-B}$ except for the designated appearance in $A \rightarrow \alpha B \beta$. Let $G^{1}$ be the resulting grammar form. Clearly $G^{\prime} \Delta_{s} G, \mathcal{L}_{s}\left(G^{\prime}, \Rightarrow\right)=\mathcal{L}_{s}(G, \Rightarrow)$ and moreover conditions (i) and (iii) are satisfied for $G^{\prime}$. Letting $G$ be the constructed $G^{\prime}$ we have the result. 


\section{3 Collections of Grammar Families}

In this section we are concerned solely with families of grammars defined by grammar forms under various interpretation mechanisms. Not only do we study g-interpretations and full ginterpretations, but we also introduce the notion of a quasiinterpretation. This is an interpretation in which the disjointness condition for nonterminals is dropped. We investigate compositions of these interpretations and their "inverses" in Sections 3.1 and 3.2 . Finally, for a given interpretation the notion of strong form equivalence is studied. It is shown that the collection of all grammar families forms a lattice in Section 3.3, and further, for each grammar form there is a unique, up to isomorphism, (production) minimal strong form equivalent grammar form in section 3.4 .

\section{II.3.1 Pre-orders and Closure operators}

Recall that a relation is a pre-order if it is both reflexive and transitive, for example the yield relation $\Rightarrow^{*}$ is a preorder. Similarly, interpretations can be viewed as relations over the class of context-free grammars, $G$. For example $\vec{g}$ is such a relation and since $G \underset{g}{a} G$ for all $G$ and $G^{\prime} \vec{g} G, G^{\prime \prime} \vec{g} G^{\prime}$ implies $G^{\prime \prime} \vec{g} G, g$ is a pre-order. Similarly $\vec{s}$ is a pre-order. This observation follows from the fact that (i) an identity substitution is a substitution, (ii) finite substitutions are closed under composition and (iji) dfisubstitutions are closed under composition.

A map $\theta: 2^{\mathscr{E}}+2^{6}$ is said to be a (grammatical) operator.

For example, consider the operator induced by $\mathbb{g}$, denoted $\theta_{g}$ defined by: for all $C \subseteq G, \theta_{g}(C)=\left\{G^{\prime} \underset{g}{\Delta} G: G\right.$ is in $\left.C\right\}$.

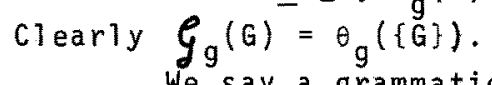

We say a grammatical operator $\theta$ is:

(i) inclusion preserving if for all $X, Y \subseteq 6$, $X \subseteq Y$ implies $\theta(X) \subseteq \theta(Y)$,

(ii) nondecreasing if for all $x \subseteq 6, x \subseteq \theta(x)$,

(iii) idempotent if for all $x \subseteq G, \theta(\theta(x))=\theta(x)$, that is, $\theta \theta=\theta$, and

(iv) union preserving if for all $X, Y \subseteq G, \theta(X \cup Y)=\theta(X) \cup \theta(Y)$. Consider ${ }^{\theta} \mathrm{g}$. Clearly it is nondecreasing since $\vec{g}$ is reflexive; it is idempotent since $\rightarrow$ is transitive; it is union preserving by definition and hence inclusion preserving. When $\theta$ is a grammatical operator and $\theta$ fulfills (i), (ii) and (iii) above, we say $\theta$ is a closure operator. Hence $\theta_{g}$ is a closure operator. 
We make precise the relationship between pre-orders and closure operators by way of the following definition.

\section{Definition}

Let $\rho$ be a relation over $\mathcal{G}$, then define the induced o-operator, $\theta_{\rho}$, by:

for a $11 c \subseteq E, \theta_{\rho}(C)=\left\{G^{\prime}: G^{\prime} \rho G\right.$ and $G$ is in $\left.C\right\}$.

Similarly given an operator $\theta$ define the induced $\theta$-relation $\rho_{\theta}$, by:

for all $G, G^{\prime}$ in $G, G^{\prime} p_{\theta} G$ iff $G^{\prime}$ is in $\theta(\{G\})$.

We now have:

\section{Lemma 3.1}

(i) A relation $\rho$ over $C_{6}$ is a pre-order iff the induced o-operator $\theta_{\rho}$ is a closure operator; in this case the induced $\theta_{\rho}$-relation equals $\rho$ and $\theta_{\rho}$ is union preserving.

(ii) An operator $\theta$ over $G$ is a union-preserving closure operator iff $\rho_{\theta}$ is a pre-order and the induced $\rho_{\theta}$-operator equals $\theta$.

Proof: This is left to the reader.

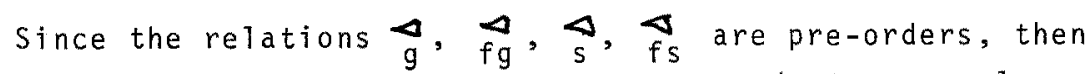
immediately the induced operators $\theta_{\mathrm{g}}, \theta_{\mathrm{fg}}, \theta_{\mathrm{s}}$ and $\theta_{\mathrm{fs}}$ are closure operators. Moreover defining the inverse operators ${ }_{\mathrm{g}}^{-1}$, etc., by:

for a $11 c \subseteq G_{0}, \theta_{x}^{-1}(C)=\left\{G: G^{\prime} \underset{x}{\longrightarrow}\right.$ and $G^{\prime}$ is in $\left.C\right\}$, where $x$ is in $\{g, f g, s, f s\}$, then it follows that the inverse operators are also closure operators.

In the following we will write $\xi_{x}$ and $\xi_{x}^{-1}$ rather than ${ }^{-1} x$ and $\theta_{x}^{-1}$, where $i t$ is assumed $\xi_{x}$ and $\xi_{x}^{-1}$ are extended to sets of grammars.

\section{II.3.2 Composition of Grammatical Closure 0perators}

Having introduced in Section 3.1 the notion of closure operators and observed their clase relationship with pre-orders, we now study in some detail compositions of closure operators of the form $\xi_{x}$ and $\xi_{x}^{-1}$, given by a pre-order $\vec{x}$. Composition is intended in the usual functional sense, for example $\xi_{x} \xi_{y}^{-1} \xi_{z}^{-1}$ is defined by:

$$
\text { for a11 } c \subseteq \mathcal{G}, \xi_{x} \xi_{y}^{-1} \xi_{z}^{-1}(c)=\xi_{x}\left(\xi_{y}^{-1}\left(\xi_{z}^{-1}(c)\right)\right) \text {. }
$$


Given a set of closure operators, $\boldsymbol{\theta}$, then we can define $\boldsymbol{U}^{*}$ to be the free monoid generated by $\boldsymbol{W}$ under composition, denoting the set of all finite compositions of operators from where the identity closure operator $\boldsymbol{g}$, is defined by

for all $c \subseteq e, g(c)=c$.

Let $0_{1}$ and $0_{2}$ be two members of $\theta *$, we say:

$0_{1} \equiv 0_{2}$ iff for all $c \leq G, 0_{1}(c)=0_{2}(c)$.

We will address the following problem:

Is $P t^{*} / \equiv$ finite?

and if it is, then provide representatives of each equivalence class.

To demonstrate that this is not always trivial we will consider the closure operators defined by four new pre-orders, which are either restrictions or generalizations of g-interpretations.

Let $G=(V, \Sigma, P, S)$ and $G^{\prime}=\left(V^{\prime}, \Sigma^{\prime}, P^{\prime}, S^{\prime}\right)$ be two grammars. We say $G^{\prime}$ is a very full g-interpretation of $G$ modulo $\mu$, if (a) $G^{\prime} \triangleleft G(\mu),\left(\right.$ b) $\mu(X) \neq \emptyset$, for all $X$ in $V$ and $(c) P^{\prime}=\mu(P)$. We denote this by $G^{\prime} \underset{\vee g}{\rightarrow} G(\mu)$. Notice that a vg-interpretation is a further restriction of a full g-interpretation. If $\mu$ is a finite letter substitution on $V-\Sigma$ and a substitution on $\Sigma$, we say $G{ }^{\prime}$ is a quasi-interpretation of $G$ modulo $\mu$, if $(a) \mu(A) \subseteq V^{\prime}-\Sigma^{\prime}$, for all $A$ in $V-\Sigma$, (b) $\mu(a) \subseteq \Sigma^{\prime *}$, for all a in $\Sigma,(c) P^{\prime} \subseteq \mu(P)$ and (d) $S^{\prime}$ is in $\mu(S)$. This is denoted by $G^{\prime} \underset{q}{a} G(\mu)$. We also define a very full quasi-interpretation, denoted $G{ }^{9} \vec{v}_{\mathrm{q}} G(\mu)$, analogousiy to the very full g-interpretation. A quasi-interpretation is similar to a g-interpretation except that different nonterminals can give rise to the same nonterminal. It is convenient to define a special interpretation, the two-symbol interpretation $G^{\prime}$ of a grammar $G$, denoted $G^{\prime} \overrightarrow{2}_{2} G(\mu)$, if $G^{\prime}$ is a twosymbol form over $\left\{S^{\prime}, a^{\prime}\right\}, \mu(A)=S^{\prime}$, for all $A^{\prime}$ in $V-\Sigma, \mu(a)=a^{\prime}$ for all a in $\Sigma$, and $P^{\prime}=\mu(P)$. Note that when $G^{\prime} \overrightarrow{2} G(\mu)$ we also have G $_{\vee q} G(\mu)$.

The q-interpretation, by its very nature, is much coarser than the g-interpretation in a sense we will now make precise.

\section{Notation}

For notational convenience in the remainder of this section we will use $\vec{x}$ synonymously with $\vec{x}$ and $\vec{x}$ synonymously with $\vec{x}$.

Lemma 3.2

$\hat{v} q g=\hat{g} v q=\hat{q}=\hat{g} 2$. 
Proof: Since $\hat{v} \dot{g}, \hat{v} q, \hat{g}$ and $\hat{2}$ are all restrictions of $\hat{q}$, we have v́q $\mathfrak{g}, \hat{g} \hat{v q}, \mathfrak{g} \hat{2} \subseteq \hat{q}$. Consider the converse inclusions. Let $G=(V, \Sigma, P, S), G^{\prime}=\left(V^{\prime}, \Sigma^{\prime}, P^{\prime}, S^{\prime}\right)$ and $G^{\prime} \mathbb{A} G(\mu)$.

(a) $\hat{q} \subseteq \hat{v} q \hat{g}$. Construct $G^{\prime \prime}=\left(V^{\prime \prime}, \Sigma^{\prime}, P^{\prime \prime}, S^{\prime \prime}\right)$, where $V^{\prime \prime}=\{[A, B]: A$ in $V-\Sigma$ and $B$ in $\mu(A)\} \cup \Sigma^{\prime}$ and $S^{\prime \prime}=\left[S, S^{\prime}\right]$. Define $\mu^{\prime \prime}$ by $\mu^{\prime \prime}(A)$

$=\{[A, B]:[A, B]: B$ in $\mu(A)\}$, for all $A$ in $V-\Sigma$ and $\mu^{\prime \prime}(a)=\mu(a)$, for all a in $\Sigma$ and define $\mu^{\prime}$ by $\mu^{\prime}(a)=\{a\}$ for all a in $\Sigma^{\prime}$ and $\mu^{\prime}([A, B])=\{B\}$, for all $[A, B]$ in $V^{*}$. Finally, choose $P^{\prime \prime} \subseteq \mu^{\prime \prime}(P)$ such that $\mu^{\prime}\left(P^{\prime \prime}\right)=P^{\prime}$, in other words $P^{\prime \prime}=\mu^{\prime-1}\left(P^{\prime}\right)$. Since $\mu^{\prime \prime}$ is a df T-substitution on $V-\Sigma, G \underset{g}{\Delta} G(\mu ")$, and since $\mu^{\prime}$ is not a dflsubstitution on $V^{\prime}-\Sigma^{\prime}$, but $P^{\prime} \stackrel{g}{=} \mu^{\prime}\left(P^{\prime \prime}\right)$ we have $G^{\prime} \overrightarrow{V q} G^{\prime \prime}\left(\mu^{\prime}\right)$. Hence the result.

(b) $\hat{q} \subseteq \hat{g} \hat{2}$. Construct $G^{\prime \prime}=(\{A, a\},\{a\}, P ", A)$ such that $G^{\prime \prime} \overrightarrow{2} G$, essentially by identifying all the terminals with a and all the nonterminals with $A$. Now define $\mu^{\prime}$ by $\mu^{\prime}(a)=\bigsqcup_{b \text { in } \Sigma} \mu(b)$ and $\mu^{\prime}(A)=\underbrace{}_{B \text { in } V-\Sigma} \mu(B)$. Immediately $P^{\prime} \subseteq \mu^{\prime}\left(P^{\prime \prime}\right)$ therefore $G^{\prime} G_{g}^{\prime \prime}\left(\mu^{\prime}\right)$ giving the required result.

(c) $\dot{q} \subseteq \dot{q} \dot{v}$. Since $\dot{2} \subseteq \dot{v} q$ the inclusion follows from $(b)$.

\section{Corozzaxy 3.3}

Let $G$ be a grammar form, then $\mathcal{Z}_{q}(G, \Rightarrow)$ is in $\{\{\emptyset\}, \mathcal{L}(F I N)$, $\mathcal{L}(\mathrm{REG}), \mathcal{L}(\mathrm{LIN}), \mathcal{L}(\mathrm{CF})\}$.

Proof: By Lemma 3.2 and Theorem 2.18 .

Thus under q-interpretations only 5 language families are obtainable. Incidentally, we have also shown that $\mathcal{G}_{\text {vq }}$ and $\mathcal{G}_{\mathrm{g}}$ commute. Since $\dot{v} g \subseteq \hat{g}, \hat{v} g \subseteq \hat{q}$ and $\hat{v} q \subseteq \hat{q}$ we have immediately that

$\hat{v g} \hat{g}=\hat{g}=\hat{g} \vee \hat{g}, \hat{v} g \hat{q}=\hat{q}=\hat{q} \vee \hat{g}$ and $\hat{v} q \hat{q}=\hat{q}=\hat{q} \hat{v} q$. Similarly since $\dot{v} g \subseteq \hat{v} q$ and $\vec{v} g \hat{v} q=\vec{v} q=\vec{v} q \dot{v} g$ each composition of two pre-orders and two operators commutes. Moreover each composition can always be reduced to one pre-order or one operator. Thus we have shown

\section{Theorem 3.3}

Let $\mathcal{U}=\left\{\mathscr{G}_{x}: x=v g, v q, g, q\right\}$ then $\mathcal{G}^{*} / \equiv$ is finite and is equal to $\left\{[\boldsymbol{g}]_{\equiv}\right\}$ u $\left\{\left[\xi_{x}\right]_{\equiv}: x=v g, v q, g, q\right\}$. Moreover $\mathcal{V}$ is a commutative semigroup under composition (see Table 3.1). 


\begin{tabular}{c|cccc} 
& $\hat{v} g$ & $\hat{v q}$ & $\hat{g}$ & $\hat{q}$ \\
\hline$\hat{v} g$ & $\hat{v g}$ & $\hat{v q}$ & $\hat{g}$ & $\hat{q}$ \\
\hline$\hat{v} q$ & $\hat{v} q$ & $\hat{v q}$ & $\hat{q}$ & $\hat{q}$ \\
\hline$\hat{g}$ & $\hat{g}$ & $\hat{q}$ & $\hat{g}$ & $\hat{q}$ \\
\hline$\hat{q}$ & $\hat{q}$ & $\hat{q}$ & $\hat{q}$ & $\hat{q}$ \\
\hline
\end{tabular}

Table 3.1: Multiplication Table for $\left\{\xi_{x}: x=v g, v q, g, q\right\}$.

By symmetry note that we have also shown that if $\mathcal{\theta}=\left\{\xi_{x}^{-1}: v g, v q, g, q\right\}$ then $\vartheta^{*} / \equiv$ is finite. Continuing our investigation we now turn to compositions of the form $\xi_{x} \xi_{y}^{-1}$ and $\xi_{x}^{-1} \xi_{y}$, alternatively, $x \hat{y}$ and $\dot{x} \hat{y}$.

We demonstrate in the following that even when $\mathcal{\theta}=\left\{\xi_{x}, \xi_{x}^{-1}: x=v g, v q, g, q\right\}, \mathscr{E} / \equiv$ is finite. However we will discover that for some $\dot{x}$ and $\dot{y}, \hat{x} \bar{y} \neq \hat{y} \hat{x}$, in other words $\dot{x}$ and $\bar{y}$ do not commute. In this case, it is not even clear that either $\bar{x} \bar{y}$ or $\hat{y} \hat{x}$ is a pre-order. For consider $\hat{x} \hat{y}$, then $x \hat{y}$ is a pre-order iff $\hat{y} \hat{x} \subseteq \hat{x} \hat{y}$. For if

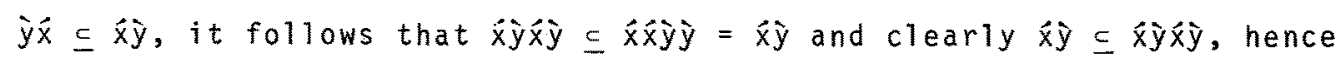
$\hat{x} \bar{y}$ is a pre-order. On the other hand if $x y y$ is a pre-order, then

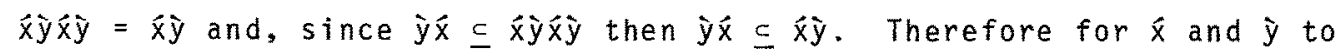
commute, in other words $\hat{x} \bar{y}=\hat{y} \hat{x}$, it is both necessary and sufficient that $\bar{x} \bar{y}$ and $\bar{y} x$ be pre-orders. Conversely, if $\hat{x}$ and $\bar{y}$ do not commute then at least one of $\hat{x} \bar{y}$ and $\bar{y} \hat{x}$ is not a pre-order.

We first obtain:

Theorem 3.4

$$
\hat{x} \bar{y}=G \times C, \text { for } x, y \text { in }\{g, q\} .
$$

Proof: Let $G_{i}=\left(V_{i}, \Sigma_{i}, P_{i}, S\right), i=1,2$ be two arbitrary grammars and $c_{1}, c_{2}$ be two new terminal symbols not in $v_{1} \cup V_{2}$. Note that we have assumed $S$ is common to $G_{1}$ and $G_{2}$. Since we are dealing with interpretations this is no $10 \mathrm{ss}$ of generality. Construct $G=\left(V_{1} \cup V_{2} \cup\left\{c_{1}, c_{2}\right\}, \Sigma_{1} \cup \Sigma_{2} \cup\left\{c_{1}, c_{2}\right\}, P, S\right)$, where $P=\left\{A \rightarrow \alpha c_{i}: A \rightarrow \alpha\right.$ is in $\left.P_{i}, i=1,2\right\}$. Defining $\mu_{i}$ by $\mu_{i}(X)=X$, for all $X$ in $V_{1} \cup V_{2}, \mu_{i}\left(c_{i}\right)=\lambda$ and $\mu_{j}\left(c_{j}\right)=\emptyset, i \neq j, i, j=1,2$, then $G_{i} \underset{g}{b}\left(\mu_{i}\right), i=1,2$. Therefore $\underset{6}{6} \boldsymbol{b} \subseteq x \bar{y}$, for all $x$, y in $\{g, q\}$, since $\dot{g} \subseteq \dot{q}$, giving the desired 
We say $G^{\prime} \overrightarrow{s u b} G$ if $G^{\prime}$ is a subgrammar of $G$. Clearly $\hat{g}=$ süb $\hat{v} g$ and $\hat{q}=$ súb $\hat{v} q$, from the definitions. We use this simple observation to simplify some of the following proofs.

Theorem 3.5

$$
\dot{x} \hat{y} \subseteq \grave{y} \hat{x} \text { for all } x, y \text { in }\{v g, v q, g, q\}
$$

Proof: Throughout we have $G_{i}=\left(V_{i}, \Sigma_{i}, P_{i}, S_{i}\right), i=1,2,3$ with $G_{2} \underset{X}{\Delta} G_{1}\left(\mu_{2}\right)$ and $G_{3} \triangleleft G_{1}\left(\mu_{3}\right)$. We wish to show there exists $G_{4}=\left(V_{4}, \Sigma_{4}, P_{4}, S_{4}\right)$ such that $G_{4} \frac{\vec{y}}{G_{2}}$ and $G_{4} \underset{x}{4} G_{3}$. Diagrammatically we have

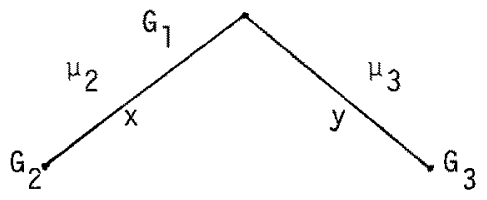

and we wish to construct $G_{4}$ such that

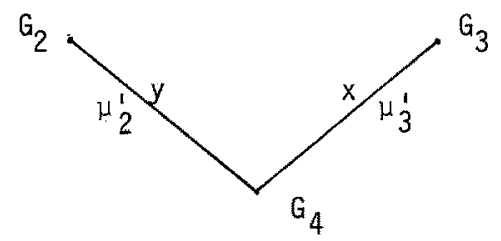

(a) $\hat{v} g$ v̀q $\subseteq$ và $\hat{v g}$. Define a relation $\psi \subseteq\left(v_{2}-\Sigma_{2}\right) \times\left(V_{3}-\Sigma_{3}\right)$ by: $A_{2} \psi A_{3}$ iff there exists an $A$ in $V_{1}-\Sigma_{1}$ with $A_{i}$ in $\mu_{j}(A), i=2,3$.

Define $\mu_{i}^{i}, i=2,3$ by $\mu_{i}^{i}(a)=\lambda$, for all a in $\Sigma_{i}, i=2,3$, and, for all $A_{2}$ in $V_{2}-\Sigma_{2}$.

$\mu_{2}^{\prime}\left(A_{2}\right)=$ either $\left\{\left[B_{2}, A_{3}\right]: B_{2} \psi A_{3}\right.$ and $\left.A_{2} \psi A_{3}\right\}$ if there is an $A$ in $V_{3}-\Sigma_{3}$ such that $A_{2} \psi A$ or $A_{2}$ otherwise,

and for all $A_{3}$ in $V_{3}-\Sigma_{3}$ :

$\mu_{3}^{\prime}\left(A_{3}\right)=$ either $\left\{\left[A_{2}, A_{3}\right]: A_{2} \psi A_{3}\right\}$ if there is an $A$ in $V_{2}-\Sigma_{2}$ such that $A \psi A_{3}$ or $A_{3}$ otherwise.

We now claim that $\mu_{2}^{1} \mu_{2}(X)=\mu_{3}^{\prime} \mu_{3}(X)$ for all $x$ in $v_{1}$ which appear in at least one production of $G_{1}$. Since $\mu_{i}$ and $\mu_{i}^{\prime}$ are very fult interpretations for $i=2,3$, if the claim is true we let $P_{4}=\mu_{2}^{\prime} \mu_{2}\left(P_{1}\right)=\mu_{3}^{1} \mu_{3}\left(P_{7}\right)$. Note that $\mu_{j}^{\prime}\left(A_{j}\right)=A_{j}$, only when $A_{j}$ does not appear in any production of $P_{i}$, hence if there is an $A$ in $V_{1}-\Sigma_{1}$ such that $A_{i}$ is in $\mu_{j}(A)$, then $A$ does not appear in any production in 
$P_{1}$ either and further $A_{i}$ does not appear in any production in $P_{4}$, $i=2,3$.

For all a in $\Sigma_{1}, \mu_{2}^{\prime} \mu_{2}(a)=\mu_{3}^{\prime} \mu_{3}(a)=\lambda$, hence $\Sigma_{4}=\emptyset$.

Consider $\left[A_{2}, A_{3}\right]$ in $\mu_{3}^{\prime} \mu_{3}(A)$, then by definition $A_{2} \psi A_{3}$ and $A_{2}$ is in $\mu_{2}(A)$, hence $\left[A_{2}, A_{3}\right]$ is in $\mu_{2}^{\prime} \mu_{2}(A)$. Conversely, if $\left[A_{2}, A_{3}\right]$ is in $\mu_{2}^{2} \mu_{2}(A)$ then by definition there exists $B_{2}$ in $\mu_{2}(A)$ and $A_{3}$ in $\mu_{3}(A)$, therefore $\left[A_{2}, A_{3}\right]$ is in $\mu_{2}^{\prime}\left(B_{2}\right)$ only if $A_{2} \psi A_{3}$, in which case $\left[A_{2}, A_{3}\right]$ is in $\mu_{3}^{\prime} \mu_{3}(A)$.

Finally let $V_{4}=\mu_{2}^{\prime}\left(V_{2}-\Sigma_{2}\right) \cup \mu_{3}^{\prime}\left(V_{3}-\Sigma_{3}\right)$ and $P_{4}=\mu_{2}^{\prime} \mu_{2}\left(P_{1}\right)=\mu_{3}^{\prime} \mu_{3}\left(P_{1}\right)$, then $G_{4} \underset{v q}{\Delta} G_{2}\left(\mu_{2}^{\prime}\right)$ and $G_{4} \underset{v g}{\Delta} G_{3}\left(\mu_{3}^{\prime}\right)$ by careful scrutiny of $\mu_{2}^{\prime}$ and $\mu_{3}^{\prime}$.

(b) $\mathbf{v g} \vee \mathrm{g} \subseteq \mathrm{v} g \mathrm{vg}$. Modify the proof in (a) replacing the definition of $\mu_{2}^{\prime}$ on nonterminals by:

For all $A_{2}$ in $V_{2}-\Sigma_{2}$,

$\mu_{2}^{\prime}\left(A_{2}\right)=$ either $\left\{\left[A_{2}, A_{3}\right]: A_{2} \Psi A_{3}\right\}$ if there is an $A$ in $v_{3}-\Sigma_{3}$ such that $A_{2} \psi A$ or $A_{2}$ otherwise.

(c) $\hat{v} q \dot{v} q \subseteq \dot{v} q \dot{v}$. Define $\mu_{j}(a)=\lambda$, for all a in $\Sigma_{i}$, and $\mu_{i}^{i}(A)=S_{4}$, for all A in $V_{i}-\Sigma_{i}, i=2,3$. clearly $\mu_{2}^{1} \mu_{2}(x)=\mu_{3}^{\prime} \mu_{3}(x)$ for all $X$ in $V_{1}$, hence letting $G_{4}=\left(\left\{S_{4}\right\}, \emptyset, \mu_{2}^{\prime} \mu_{2}\left(P_{1}\right), S_{4}\right)$ we have $G_{4} \underset{\vee q}{\Delta} G_{i}\left(\mu_{i}^{i}\right), i=2,3$.

(d) v́q sùb $\subseteq$ sùb viq. Since $G_{3} \overleftrightarrow{\text { sub }} G_{1}\left(\mu_{3}\right), \mu_{2}$ is well defined on $V_{3} \subseteq V_{1}$, hence letting $G_{4}=\left(V_{2}, \Sigma_{2}, \mu_{2}\left(P_{3}\right), S_{2}\right)$ implies $G_{4} \underset{V q}{\rightarrow} G_{3}$ and $G_{4} \underset{\text { sub }}{ } G_{2}$, since $\mu_{2}\left(P_{3}\right) \leq \mu_{2}\left(P_{1}\right)=P_{2}$.

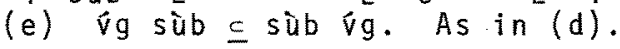

To complete the theorem we use the observation that $\hat{q}=s u b$ va and $\dot{g}=$ súb viq. For example, given

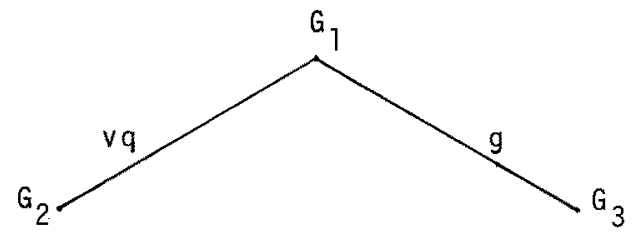

then we know there exists $G_{j}^{\prime}$ such that

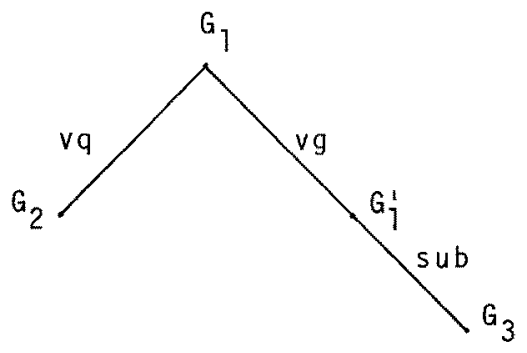


Now by (c) above we obtain:

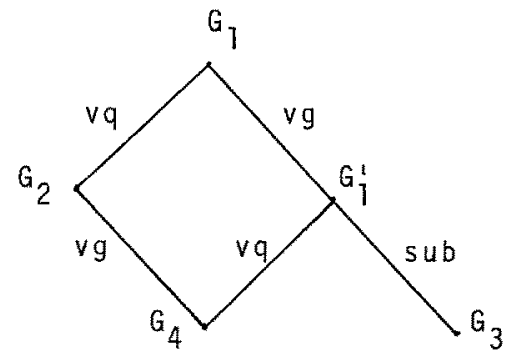

and by (d) we can complete this to give

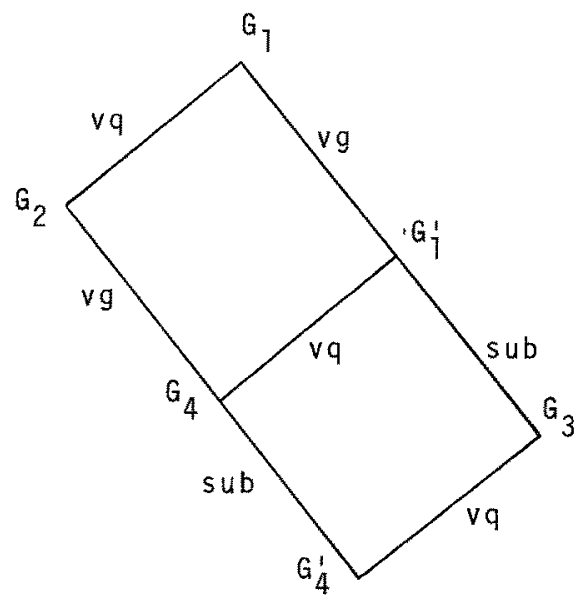

and therefore $G_{4} \underset{g}{\Delta} G_{2}$ and $G_{4} \underset{\vee q}{\Delta} G_{3}$ as desired giving $\forall q$ We can carry out the diagram chasing for all other cases bar those $x \hat{x}$, where $x, y$ are in $\{g, q\}$. However in any of these cases let $G_{4}=(\{S\}, \emptyset, \emptyset, S)$ whence $\ddot{x} \hat{y} \subseteq \bar{y} x$.

We summarize the results so far in Table 3.2 .

\begin{tabular}{c|cccc} 
& $\hat{v} g$ & $\grave{v} q$ & $\grave{g}$ & $\grave{q}$ \\
\hline$\hat{v} g$ & $c$ & $c$ & $c$ & $c$ \\
\hline$\dot{v} q$ & $c$ & $c$ & $c$ & $c$ \\
\hline$\hat{g}$ & $c$ & $c$ & $\bar{u}$ & $\overline{\bar{u}}$ \\
\hline$\dot{q}$ & $c$ & $c$ & $\bar{u}$ & $\bar{u}$ \\
\hline
\end{tabular}

c indicates $\bar{x} \bar{y} \subseteq \dot{y} x$

$\overline{\bar{u}}$ indicates $\hat{x} \bar{y}=\bar{y} \hat{x}=\ell \times \ell$, the universal relation 
We have used symmetry to fill out this table and the fact that since $\overline{\bar{u}}=\hat{x} \hat{y}$, for all $x, y$ in $\{g, q\}$ and $\hat{x} \hat{y} \subseteq \hat{y} \hat{x}$, then $\hat{x} \hat{y}=\hat{y} \hat{x}=\bar{u}$. We turn now to the consideration of the relations $\ddot{x} \bar{y}$, for all $x$, y in $\{v g, v q, g, q\}$.

For consistency, we will assume that we are given

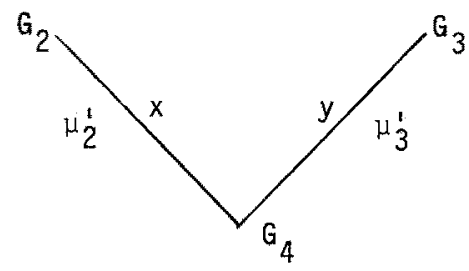

in each of the following theorems for which the inclusion $\dot{x} \bar{y} \subseteq \hat{y} \bar{x}$ is to be proved.

\section{Theorem 3.6}

vig $\hat{y} \nsubseteq \hat{y} \dot{v} g$, for $y=v g$ and $v q$.

Proof: We first prove a stronger result, namely v̀gíg $\notin$ viqùg. Let $G_{2}, G_{3}, G_{4}$ be defined by the productions:

$$
\begin{aligned}
& G_{2}: S \rightarrow B a C ; S \rightarrow B \bar{C} \\
& G_{3}: S \rightarrow B a C ; S+\overline{B C} \\
& G_{4}: S \rightarrow B C ; S \rightarrow \overline{B C} ; S \rightarrow B C ; S \rightarrow \bar{B} \bar{C} .
\end{aligned}
$$

Clearly $G_{4} \underset{v g}{ } G_{i}, i=2,3$.

Now assume there exists $G_{1}$ such that $G_{2} \overrightarrow{v q} G_{1}\left(\mu_{2}\right)$ and $G_{3} \underset{v g}{A} G_{1}\left(\mu_{3}\right)$. Without loss of generality we may assume $G_{1}$ is reduced. Clearly $V_{1}$ consists of at least 4 nonterminals and one terminal, since assuming otherwise implies

either $\mu_{3}(D)$ contains at least two nonterminals from

$V_{3}-\Sigma_{3}$ for some $D$ in $V_{1}-\Sigma_{1}$, in which case since

$G_{1}$ is reduced, both must appear in the productions of

$G_{1}$ and hence $\mu_{3}\left(P_{1}\right) \neq P_{3}$, whatever the choice, a

contradiction,

or $G_{1}$ has no terminals, in which case $P_{3}$ has no

terminals, a contradiction.

Further $V_{1}$ contains exactly 4 nonterminals, since $G_{1}$ is reduced and nonterminals are preserved under vg-interpretation, hence let them be denoted $S, B, \bar{B}, C$ where $\mu_{3}$ is the identity on $V_{1}-\Sigma_{1}$, without loss of generality. 
$\mu_{2}\left(P_{1}\right)$, by definition. It is clear that $G_{2} \underset{g}{a} G_{1}\left(\mu_{2}\right)$ if $y=g$ and $G_{2} \vec{q} G_{1}\left(\mu_{2}\right)$ otherwise.

Our final result is:

Theorem 3.8

v̀ $\bar{y} \subseteq \bar{y}$ v̀ for $y=v q, g$ and $q$.

Proof: We will first prove ìq viq $\subseteq$ viq v̀q.

(a) Let $W_{i}=\left\{x: x\right.$ in $\Sigma_{i}^{*}, A \rightarrow \alpha x \beta$ is in $P_{i}, \alpha \beta$ in $\left.V_{i}^{*}\right\}, i=2,3$

and let $G_{1}=\left(V_{1}, \Sigma_{1}, P_{1}, S_{1}\right)$ be defined as follows:

$$
\begin{aligned}
v_{1}- & \Sigma_{1}=\left\{\left[A_{2}, A_{3}\right]: A_{i} \text { in } v_{i}-\Sigma_{i}, i=2,3\right\}, \\
\Sigma_{1}= & \left\{\left[x_{2}, x_{3}\right]: x_{i} \text { in } W_{i}, i=2,3\right\}, \\
P_{1}= & \left\{[A, B] \rightarrow\left[x_{0}, y_{0}\right]\left[A_{1}, B_{1}\right] \ldots\left[A_{n}, B_{n}\right]\left[x_{n}, y_{n}\right]:\right. \\
& \left.A \rightarrow x_{0} A_{1} \ldots x_{n} \text { is in } P_{2}, B \rightarrow y_{0} B_{1} \ldots B_{n} \text { is in } P_{3}\right\},
\end{aligned}
$$

and

$$
s_{1}=\left[s_{2}, s_{3}\right] \text {. }
$$

Let $\mu_{2}$ and $\mu_{3}$ be defined by:

$$
\mu_{i}\left(\left[x_{2}, x_{3}\right]=x_{i} \text {, for } i=2,3 \text {, for all }\left[x_{2}, x_{3}\right] \text { in } v_{1}\right. \text {. }
$$

Now for each $\mathrm{p}: C \rightarrow z_{0} C_{1} \ldots C_{n} z_{n}$ in $P_{4}$ there are productions

$q: A \rightarrow x_{0} A_{1} \ldots A_{n} x_{n}$ and $r: B \rightarrow y_{0} B_{1} \ldots B_{n} y_{n}$ in $P_{2}$ and $P_{3}$

respectively, such that $p$ is in $\mu_{2}^{\prime}(q) n \mu_{3}^{\prime}(r)$. Conversely, given $q$ in $P_{2}$, there is a $p$ in $P_{4}$ and an $r$ in $P_{3}$ satisfying $p$ is in $\mu_{2}^{\prime}(q) n \mu_{2}^{\prime}(r)$, and similarly for each $r$ in $P_{3}$. Hence $P_{1}$ as defined contains encoded versions of both $\mathrm{P}_{2}$ and $\mathrm{P}_{3}$ which are recovered by the substitutions $\mu_{2}$ and $\mu_{3}$, respectively. Therefore

$$
G_{i} \underset{v q}{\rightarrow} G_{1}\left(\mu_{i}\right), i=2,3 \text {. }
$$

(b) $\dot{\mathrm{v} q} \subseteq \dot{\mathrm{g}} \mathrm{v} q$. Proceed as in (a) except that $P_{1}$ should also include

$$
\begin{aligned}
& \left\{\left[A_{0}, B_{0}\right] \rightarrow\left[x_{0}, y_{0}\right]\left[A_{1}, B_{1}\right] \ldots\left[A_{n}, B_{n}\right]\left[x_{n}, y_{n}\right]:\right. \\
& B_{0} \rightarrow y_{0} B_{1} \ldots B_{n} y_{n} \text { is in } P_{3}, A_{i} \text { is in } v_{2}-\Sigma_{2}, \text { and } x_{j} \text { is in } w_{2}, \\
& 0 \leq i \leq n\} .
\end{aligned}
$$

Clearly $G_{3} \underset{v q}{ } G_{1}\left(\mu_{3}\right)$ with $\mu_{3}$ as in (a). We need to show that $G_{2} \underset{g}{g} G_{1}$. We define $\mu_{2}$ as follows:

For each $A$ in $V_{2}-\Sigma_{2}$ which appears in a production in $P_{2}$, select just one $B$ in $V_{3}-\Sigma_{3}$ with $\mu_{2}^{\prime}(A) \cap \mu_{3}^{\prime}(B) \neq \emptyset$; if $A$ does not appear in any production choose $B$ arbitrarily from $V_{3}-\Sigma_{3}$. Let $\mu_{2}([A, B])=A$ if $B$ has been selected for $A$ and $\mu_{2}([A, B])=\emptyset$ otherwise, and let $\mu_{2}([x, y])=\{x\}$, for all $[x, y]$ in $\Sigma_{1}$. As in $(a)$, for each $q$ in $P_{2}$ there is a $p$ in $P_{4}$ with $p$ in $\mu_{2}^{i}(q)$ and an $r$ in $P_{3}$ with $p$ in $\mu_{3}^{\prime}(r)$. 
Consider $P_{1}$. Since $V_{1}-\Sigma_{1}$ consists of four nonterminals, then $P_{1}$ contains only productions of two types, namely,

$$
S \rightarrow x B y C z
$$

and

$$
S \rightarrow U \bar{B} \vee C W
$$

where $u, v, w, x, y, z$ are terminal words.

Turning to $G_{2}$ we now obtain a contradiction. Clearly $S$ is in $\mu_{2}(S)$ and $\{C, \bar{C}\} \subseteq \mu_{2}(C)$. Since $G_{2} \underset{\vee q}{G} G_{1}\left(\mu_{2}\right)$ then $S \rightarrow B a C$ is in $\mu_{2}\left(P_{1}\right)$ and further, there is a production $p: S \rightarrow x X y C z$ in $P_{1}$ such that

$$
S \rightarrow B a C \text { is in } H_{2}(p) \text {. }
$$

However this implies that $S \rightarrow B a \bar{C}$ is also in $\mu_{2}(p)$ and hence in $P_{2}$, a contradiction. Therefore $G_{1}$ does not exist. Hence $\dot{v}$ i $g \subseteq \forall \mathbf{q} \dot{v}$.

To complete the theorem observe that:

$\hat{v} g \hat{v} g \subseteq \hat{v} q \dot{v} g$ therefore $\dot{v} g \hat{v} g \notin \hat{v} g \hat{v} g$, and

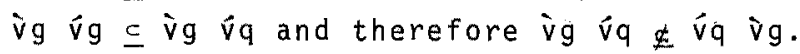

Having established this non-inclusion result, it then follows that we cannot make use of diagram extension to obtain results for $\mathbf{v g} \hat{g}$ and ìg $\ddot{q}$. Therefore we treat these cases separately.

Theorem 3.7

ìg $\hat{y} \subseteq \hat{y}$ v̀g for $y=g$ and $q$.

Proof: Without loss of generality we will assume $G_{4}$ has no terminal symbols. Define a homomorphism $h$ by $h(A)=A$, for all nonterminals $A$ in $V_{2} \cup V_{3}$ and $h(a)=\lambda$, for all terminals a in $\Sigma_{2} \cup \Sigma_{3}$. Since $\mu_{2}^{1}$ is a very full interpretation we can assume $A$ is in $\mu_{2}^{\prime}(A)$, for all $A$ in $V-\Sigma_{2}$. Hence for all $p$ in $P_{2}$ there exists $q$ in $P_{4}$ such that $h(p)=q$.

Construct $G_{1}=\left(V_{1}, \Sigma_{1}, P_{1}, S_{1}\right)$ from $G_{3}$ as follows:

$V_{1}-\Sigma_{1}=V_{3}-\Sigma_{3}, \Sigma_{1}=\Sigma_{3} u\{c\}$, where $c$ is a new symbol not in $V_{3}$, $P_{1}=\left\{A \rightarrow \alpha_{1} c \alpha_{2} \ldots \alpha_{n} c: A+\alpha_{1} \ldots \alpha_{n}\right.$ is $P_{3}, n \geq 1, \alpha_{1}$ is in $\Sigma_{3}^{*}$, $\alpha_{i}$ is in $\left.\left(V_{3}-\Sigma_{3}\right) \Sigma * x, 1<i \leq n\right\}$, and $s_{1}=s_{3}$.

Clearly $G_{3} \underset{v g}{A} G_{1}\left(\mu_{3}\right)$ where $\mu_{3}(x)=x$, for all $x$ in $V_{3}$ and $\mu_{3}(c)=\lambda$. Define $\mu_{2}$ by:

$$
\begin{aligned}
\mu_{2}(A)= & \mu_{3}^{\prime}(A), \text { for all } A \text { in } V_{1}-\Sigma_{1}=V_{3}-\Sigma_{3}, \text { and } \\
\mu_{2}(a)= & \left\{x: x \text { is in } \Sigma_{2}^{*} \text { and } B \rightarrow \alpha x \beta \text { is in } P_{2},\right. \text { for some } \\
& \left.\alpha, \beta \text { in } V_{2}^{*}\right\}, \text { for all a in } \Sigma_{1} .
\end{aligned}
$$

Then $P_{2} \subseteq \mu_{2}\left(P_{1}\right)$ since $\lambda$ is in $\mu_{2}(a)$ for all a and for all $p$ in $P_{2}$ there exists $q$ in $P_{4}$ with $h(p)=q$, which in turn implies there is $r$ in $P_{3}$ with $q$ in $\mu_{3}^{\prime}(r)$ and $r^{\prime}$ in $P_{1}$ with $h\left(r^{\prime}\right)=h(r)$, hence $p$ is in 
therefore $q$ can be recovered from $P_{1}$. Hence $q$ is in $\mu_{2}\left(P_{1}\right)$, and $\mathrm{G}_{2} \underset{\mathrm{g}}{\Delta} \mathrm{G}_{1}\left(\mu_{2}\right)$ by definition of $\mu_{2}$.

(c) As in (b) defining $\mu_{2}$ by:

$\mu_{2}([X, Y])=X$ for a $11[X, Y]$ in $V_{1}$,

clearly by the above arguments $G_{2} \underset{q}{a} G_{1}\left(\mu_{2}\right)$.

We are now in a position to extend Table 3.2 considerably, completing our investigation of the compositions of two pre-orders. The result is seen in Table 3.3. By our earlier remarks this means that the pairs $(\hat{v} g, \hat{v g}),(\hat{v} g, \hat{v} q)$ and $(\hat{v} q, \hat{v g})$ are not commuting pairs. Similarly, we know that $\mathbf{v}$ vेg is not a pre-order

\begin{tabular}{c|cccc} 
& $\hat{v g}$ & $\hat{v q}$ & $\hat{g}$ & $\hat{q}$ \\
\hline$\hat{v} g$ & $\not$ & $\not$ & $=$ & $=$ \\
\hline$\dot{v} q$ & $\not$ & $=$ & $=$ & $=$ \\
\hline$\grave{g}$ & $=$ & $=$ & $\overline{\bar{u}}$ & $\overline{\bar{u}}$ \\
\hline$\grave{q}$ & $=$ & $=$ & $\overline{\bar{u}}$ & $\overline{\bar{u}}$ \\
\hline
\end{tabular}
where $\not$ means $\dot{x} \hat{y} \notin \dot{y} \hat{x}$ but $\hat{y} \bar{x} \subseteq \hat{x} \hat{y}$.

\section{Table 3.3}

and neither are $\mathbf{v} q \bar{v} g$ and $\bar{v} g \hat{v} q$. In terms of operators this means that $\xi_{v g}^{-1}, \quad \xi v g$ do not commute, and similarly for the others.

Let us turn to the composition of three pre-orders. Since by

Table 3.1 we can always replace or simplify $\ddot{x} \hat{y}$ by a single pre-order, and similarly with $\bar{x} \bar{y}$ (by symmetry), we only need cansider compositions of the forms

xyyz or $\bar{x} y \hat{z}$

and by symmetry we only need consider one or the other.

Consider $\hat{x} \bar{y} z$. If $\dot{x}$ and $\hat{y}$ commute (see Table 3.3 ) we can replace $x \hat{y} z$ by $\mathbf{y} \hat{x} z$ and this in turn, using Table 3.1 , by $\mathbf{y} w$. Similarly if $\bar{y}$ and $z$ commute we obtain $\mathbf{x} y$. This only leaves those combinations for which neither $\dot{x}, \hat{y}$ nor $\hat{y}, \hat{z}$ commute. These are

(i) $\hat{v g}$ v̀ $\mathrm{vg}$;

(iii) $\mathrm{vg}$ vे $\mathrm{vg}$;

(ii) $\hat{v g}$ v̀ $\hat{v q}$;

(iv) $\hat{\mathrm{q}} \mathrm{v} \mathrm{g} \mathrm{vg}$;

(v) $\mathrm{vq} \mathrm{v} g$ vq.

Now using the general transformation $\hat{x} \bar{y} z \subseteq \bar{y} \dot{x} z$ which is valid by Table 3.2, we obtain $\hat{x} \hat{y} \hat{z} \subseteq \hat{y} \hat{w}$ and if $w=z$ then $\hat{x} \hat{y} \hat{z}=\hat{y} \hat{z}$. This is the case for (i), (ii), (iji) and (v), giving (i) vig vig (ii) v̀ v́q

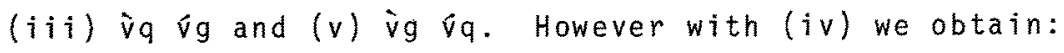

v́q ìg $r g \subseteq \grave{v} g$ vq.

Thus it remains to prove that the reverse inclusion holds even in this case. 
Let $G=(V, \Sigma, P, S)$ be a grammar, then we say $X$ in $V$ is an extra symbol if it does not appear in any production in $P$. We say $G$ has extra symbols if $V$ contains at least one extra symbol. To complete the investigation of compositions of three pre-orders we need:

\section{Theorem 3.9}

(i) Let $G=(V, \Sigma, P, S)$ be a grammar form, then there exists a grammar form $G_{1}$ with no extra symbols such that $G \underset{v g}{A} G_{1}$.

(ii) Let $G_{i}=\left(V_{i}, \emptyset, P_{i}, S_{i}\right), i=1,2$ be two grammar forms with no extra symbols and $G_{1} \underset{v q}{A} G_{2}(\mu)$. Then there exists a grammar form $G$ such that $G \underset{\vee g}{\Delta} G_{i}, i=1,2$.

Proof: (i) Let $G_{1}=\left(V_{1}, \Sigma_{1}, P, S\right)$ where $V_{1} \subseteq V, \Sigma_{1} \subseteq \Sigma$ and $V-V_{1}$ is exactly the extra symbols of $V$. Clearly $G \underset{V g}{G} G_{1}$, by the definition of interpretation.

(ii) Let $G=(V, \emptyset, P, S)$ where $V=\left\{[A, B]\right.$ : $A$ in $V_{1}, B$ in $V_{2}$ and $A$ is in $\mu(B)\}$. Define $\mu_{2}$ on $V_{2}$ by:

$$
\mu_{2}(B)=\{[A, B]: A \text { is in } \mu(B)\} \text {, }
$$

let $P=\mu_{2}\left(P_{2}\right)$ and $S=\left[S_{1}, S_{2}\right]$, then clearly $G \underset{V g}{A} G_{2}\left(\mu_{2}\right)$.

Similarly define $\mu_{1}$ on $v_{1}$ by:

$$
\mu_{1}(A)=\{[A, B]: \text { is in } \mu(B)\} \text {. }
$$

We need to show that $G \underset{\vee g}{\Delta} G_{1}\left(\mu_{1}\right)$.

It suffices to prove that $\mu_{1}\left(P_{1}\right)=\mu_{2}\left(P_{2}\right)$. Without loss of generality we may assume $P_{1} \neq \emptyset$.

(a) $\mu_{1}\left(P_{1}\right) \subseteq \mu_{2}\left(P_{2}\right)$.

Assume $A_{0} \rightarrow A_{1} \ldots A_{m}$ is in $P_{1}$, then there exist $B_{0}, B_{1}, \ldots, B_{m}$ with $A_{j}$ in $\mu\left(B_{j}\right), 0 \leq i \leq m$ such that

$$
A_{0} \rightarrow A_{1} \ldots A_{m} \text { is in } \mu\left(B_{0} \rightarrow B_{1} \ldots B_{m}\right)
$$

hence $\left[A_{0}, B_{0}\right] \rightarrow\left[A_{1}, B_{1}\right] \ldots\left[A_{m}, B_{m}\right]$ is in $\mu_{1}\left(P_{1}\right)$ and also in $\mu_{2}\left(P_{2}\right)$.

(b) $\mu_{2}\left(P_{2}\right) \subseteq \mu_{1}\left(P_{1}\right)$ follows similarly.

We now apply Theorem 3.9 to give:

\section{Theorem 3.10}

$$
\hat{v} q \grave{v} g \hat{v} g=v \grave{g} \hat{v} q
$$

Proof: Since we have already shown that vị vig $\hat{v} g \subseteq \hat{v}$ viq it remains to prove the reverse inclusion. 
craim 1: Let $G_{i}=\left(V_{i}, \Sigma_{i}, P_{j}, S_{j}\right) i=1,2$ be such that $G_{1} \underset{x}{ } G_{2}(u)$, where $x=v g$ or $v q$, then there exists $G_{i}^{\prime}=\left(V_{i}^{1}, \emptyset, P_{j}^{1}, s_{j}\right), i=1,2$ with $G_{i}^{\prime} \underset{v g}{\Delta} G_{i}, G_{i}^{\prime} \underset{x}{\Delta} G_{2}^{\prime}\left(\mu^{\prime}\right)$, and $V_{i}^{\prime}=V_{i}-\Sigma_{i}, i=1,2$.

Simply define $\mu_{j}, i=1,2$ by:

$\mu_{i}(a)=\lambda$, a in $\Sigma_{i}$ and $\mu_{i}(A)=A$, A in $V_{i}-\Sigma_{j}$,

then $G_{j}^{\prime} \triangle \vec{V} G_{i}\left(\mu_{i}\right)$ and moreover restricting $\mu$ to the nonterminals of $V_{2}$ giving $\mu^{\prime}$ implies

$$
G_{1}^{\prime} \rightarrow G_{2}^{\prime}\left(\mu^{\prime}\right)
$$

in other words $\mu_{1} \mu\left(P_{2}\right)=\mu^{\prime} \mu_{2}\left(P_{2}\right)$.

Claim 2: Let $G_{i}=\left(V_{i}, \emptyset, P_{i}, S_{i}\right), i=1,2$ be such that $G_{1} \vec{x} G_{2}(\mu)$ for $x=v g$ or $v q$, then there exists $G_{i}^{\prime \prime}=\left(V_{j}^{\prime \prime}, D, P_{j}^{\prime \prime}, S_{j}\right), i=1,2$ with $G_{i} \overrightarrow{V g} G_{i}^{\prime \prime}, i=1,2, G_{1}^{\prime \prime} \vec{x} G_{2}^{\prime \prime}\left(\mu^{\prime \prime}\right)$, and $V_{i}^{\prime \prime}=V_{i}$ - the extra nonterminals in $\left.G_{i}\right\}, i=1,2$.

By Theorem $3.9 G_{1}^{\prime \prime}$ and $G_{2}^{\prime \prime}$ exist, hence define $\mu^{\prime \prime}$ by:

$$
\mu^{\prime \prime}(A)=\mu(A) \text {, for all } A \text { in } V_{2}^{\prime \prime} \text {, }
$$

and immediately $G_{1}^{\prime \prime} \underset{x}{\Delta} G_{2}^{\prime \prime}\left(\mu^{\prime \prime}\right)$.

$$
\text { Now consider } G_{i}=\left(V_{i}, \Sigma_{i}, P_{i}, S_{i}\right), i=1,2,3 \text { such that }
$$

$G_{2} \underset{\vee g}{\Delta} G_{3}\left(\mu_{3}\right)$ and $G_{2} \underset{v q}{\forall} G_{1}\left(\mu_{1}\right)$, then diagramatically we have:

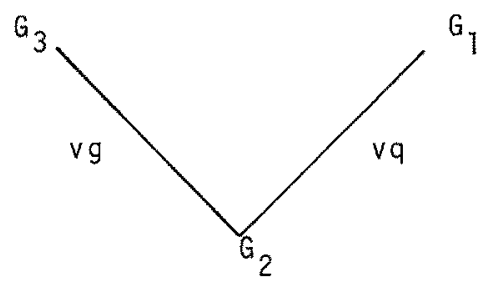

which by the above two claims we can transform into:

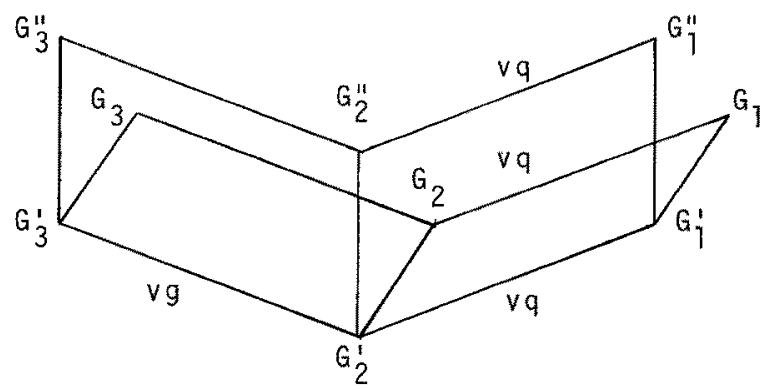


where the $G_{i}^{\prime \prime}$ have no terminals and no ext.ra symbols, $G_{2}^{\prime \prime} \underset{v g}{\Delta} G_{3}^{\prime \prime}\left(\mu_{3}^{\prime \prime}\right)$, $G_{2}^{\prime \prime} \triangleleft G_{1}^{\prime \prime}\left(\mu_{p}^{\prime \prime}\right)$ and all unmarked edges are vg-interpretations.

Since $G_{1}^{\prime \prime}$ and $G_{2}^{\prime \prime}$ fulfill the conditions of part (ii) of

Theorem 3.9 then we can construct $G$ such that

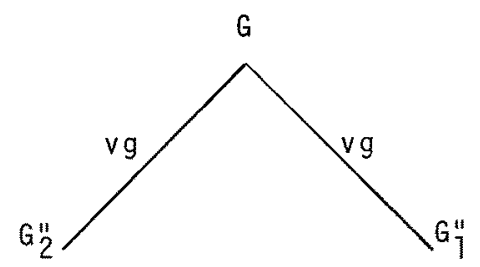

We can now relate $G_{3}$ and $G_{1}$ as follows:

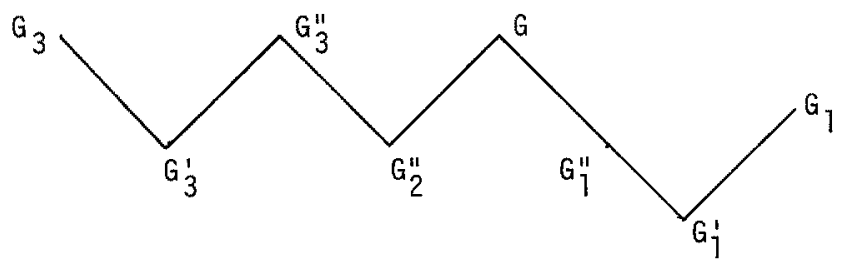

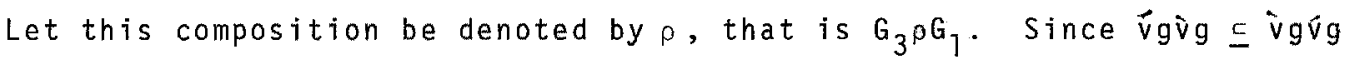
and $\hat{v} g \hat{v} g=\hat{v} g$ we obtain

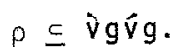

By construction v̀griq $\subseteq \rho$ and ìgriq $\subseteq$ viqùgíg, hence the result.

We have demonstrated as a result of Theorem 3.10 the following:

\section{Theorem 3.11}

Let $\theta=\left\{g_{x}, \xi_{x}^{-1}: x=g, q, v g, v q\right\}$. Then $\mathcal{E}^{*} / \equiv$ is finite.

To complete this investigation it is necessary to exhibit the equivalence classes of $\theta$. This involves proving that the various "irreducible" compositions considered are indeed distinct. This straightforward but laborious task we leave to the reader.

To close this section we give an example of two pre-orders $\hat{x}, \bar{y}$ for which $\bar{x} \hat{y} \notin \bar{y} \hat{x}$ and $\hat{y} \hat{x} \notin \hat{x} \hat{y}$, thus demonstrating that $\vartheta$ is not 
always as nicely behaved as those considered in this section. Let fg denote a full g-interpretation. Consider the pair ig and fo. As in Theorem 3.6 we have $\overline{\mathrm{g}} \dot{\mathrm{f} g} \notin$ fgig. Now consider the grammars:

$$
\begin{aligned}
& G: S \rightarrow S S ; S \rightarrow S a \\
& G_{1}: S \rightarrow S S ; \\
& G_{2}: S \rightarrow S S ; S \rightarrow S
\end{aligned}
$$

Then $G_{1} \underset{f g}{\Delta} G$ and $G_{2} \underset{v g}{\Delta} G$ but there is no $F$ with $F \overrightarrow{v g} G_{1}$ and $F \underset{f g}{\Delta} G_{2}$, hence $\hat{f} g \hat{v} g \notin \hat{v} g \hat{f g}$.

\section{II.3.3 Strong Form Equivalence and Lattices}

Two grammar forms $G_{1}$ and $G_{2}$ are strong $x$-form equivalent if $\xi_{x}\left(G_{1}\right)=\xi_{x}\left(G_{2}\right)$. We write $G_{1} \equiv{ }_{x} G_{2}$. Immediately $G_{1} \equiv_{x} G_{2}$ iff $G_{1} \vec{X} G_{2}$ and $G_{2} \vec{X} G_{1}$. It is easily verified that $\equiv_{X}$ is an equivalence relation over $\mathcal{E}$, hence for each grammar form $G$, let [G]x denote the equivalence class containing $G$ modulo $\equiv_{X}$. For each grammar form $G$, let $\mathcal{E}_{x}(G)=\left\{\left[G^{\prime}\right]_{X}: G^{\prime} \vec{x}_{x} G\right\}$ and $\boldsymbol{E}_{x}=\left\{[G]_{X}: G\right.$ in $\boldsymbol{G}_{\}}$. Define an "interpretation" relation $\leq_{x}$, over $\varepsilon_{x}$ by: for all $E_{1}$, $E_{2}$ in $\boldsymbol{E}_{x}$, $E_{1} \leq_{x} E_{2}$ if there exist $G_{1}$ and $G_{2}$ with $E_{i}=\left[G_{i}\right]_{x}, i=1,2$ and $G_{1} \stackrel{x}{ } G_{2}$.

The relation $\leq_{x}$ is again a pre-order. Further since $E_{1} \leq_{x} E_{2}$ and $E_{2} \leq_{x} E_{1}$ implies $E_{1}=E_{2}$, $\leq_{x}$ is anti-symmetric and therefore a partial order. We show in the following that $\left(\boldsymbol{E}_{x}, \leq_{x}\right)$ and $\left(\boldsymbol{E}_{x}(G), \leq_{x}\right)$ are distributive lattices.

\section{Definition}

Let $M$ be a set, partially ordered under $\leq$. An element $z$ in $M$ is said to be a greatest lower bound ( $g l b)$ of the elements $x, y$ in $M$, denoted by $z=x \wedge y$, if $z \leq x, z \leq y$ and $z^{\prime} \leq z$ for every element $z^{\prime}$ in $M$ satisfying $z^{\prime} \leq x$ and $z^{\prime} \leq y$. An element $z$ in $M$ is said to be a least upper bound (1ub) of the elements $x, y$ in $M$ denoted $z=z \vee y y$ if $z \geq x, z \geq y$ and $z^{\prime} \geq z$ for every element $z^{\prime}$ in $M$ satisfying $z^{\prime} \geq x$ and $z^{\prime} \geq y$. A lattice is a pair $(M, s)$, where $M$ is a set partially ordered under s and each pair of elements of $M$ have both a glb and an lub. A lattice $(M, \leq)$ is distributive if

$$
x \wedge(y \vee z)=(x \wedge y) \vee(x \wedge z)
$$

holds for all elements $x, y$ and $z$ in $M$.

In the remainder of this section we assume $x=s$ throughout, that is we only deal with strict interpretations. The interested reader can prove the analogous results for the other interpretations. 
We begin by proving that the intersection of two grammar families is a grammar family.

Lemma 3.12

For a 11 grammar forms $G_{1}$ and $G_{2}$ there exists a grammar form $G$ satisfying $\xi_{s}(G)=\xi_{s}\left(G_{1}\right) \cap \xi_{s}\left(G_{2}\right)$.

Proof: Let $G_{i}=\left(V_{i}, \Sigma_{j}, P_{i}, S_{i}\right), i=1,2$. Construct a grammar form $G=(V, \Sigma, P, S)$ as follows:

$V=\left(\left(V_{1}-\Sigma_{1}\right) \times\left(V_{2}-\Sigma_{2}\right)\right) \cup\left(\Sigma_{1} \times \Sigma_{2}\right)$

$\Sigma=\Sigma_{1} \times \Sigma_{2}$,

$S=\left[S_{1}, S_{2}\right]$, and $P$ consists of the following productions:

for each production $A \rightarrow X_{1} \ldots X_{n}$ of $P_{1}$

and each production $B \rightarrow Y_{1} \ldots Y_{n}$ of $P_{2}$

such that $\left[X_{i}, Y_{j}\right]$ is in $V, 1 \leq i \leq n$ take the production:

$[A, B] \rightarrow\left[X_{1}, Y_{1}\right] \ldots\left[X_{n}, Y_{n}\right]$ into $P$.

The condition $\left[X_{i}, Y_{j}\right]$ in $V$ ensures that nonterminals are paired with nonterminals and terminals with terminals. We now prove that $\xi_{s}(G)$ is indeed equal to $\xi_{s}\left(G_{1}\right) \cap \xi_{s}\left(G_{2}\right)$. consider $\xi_{s}(G) \subseteq \xi_{s}\left(G_{1}\right) \cap \xi_{s}\left(G_{2}\right)$. We show that $G \underset{S}{\Delta} G_{1}$ which implies by transitivity that

$\boldsymbol{G}_{s}(G) \subseteq \boldsymbol{G}_{s}\left(G_{1}\right)$. Consider a finite substitution $\mu$ defined on $V_{1}^{*}$ by $\mu(A)=\{A\} \times\left(V_{2}-\Sigma_{2}\right)$ and $\mu(a)=\{a\} \times \Sigma_{2}$, for each $A$ in $V_{1}-\Sigma_{1}$ and each a in $\Sigma_{1}$. Observe that $\mu(X) \cap \mu(Y)=\emptyset$ for $X \neq Y$, and $\left[s_{1}, s_{2}\right]$ is in $\mu\left(s_{1}\right)$. Let $p:[A, B] \rightarrow\left[x_{1}, y_{1}\right] \ldots\left[x_{n}, y_{n}\right]$ be an arbitrary production of $P$. Now $p_{1}: A \rightarrow X_{1} \ldots x_{n}$ is in $P_{l}$, by construction, hence $p$ is in $\mu\left(p_{1}\right)$. Therefore $G \underset{s}{ } G_{1}(\mu)$. We can prove similarly that $G \underset{s}{a} G_{2}$ hence $\xi_{s}(G)$ is in both $\xi_{s}\left(G_{1}\right)$ and $\xi_{s}\left(G_{2}\right)$. We turn to the reverse inclusion. Suppose $G^{\prime}=\left(V^{\prime}, \Sigma^{\prime}, P^{\prime}, S^{\prime}\right)$ is in $\xi_{s}\left(G_{1}\right) \cap \xi_{s}\left(G_{2}\right)$. There exist substitutions $\mu_{1}$ and $\mu_{2}$ such that $G^{\prime} \vec{s}_{i} G_{j}\left(\mu_{i}\right), \stackrel{i}{=}=1,2$. Let $u$ be the substitution on $V$ defined by:

$$
\left.\mu([X, Y])=\mu_{1}(X) \cap \mu_{2}(Y) \text { for a } 1\right][X, Y] \text { in } V \text {. }
$$

Note that $S^{\prime}$ is in $\mu\left(\left[S_{1}, S_{2}\right]\right)=\mu_{1}\left(S_{1}\right) \cap \mu_{2}\left(S_{2}\right)$. Moreover

$$
\begin{aligned}
& \mu\left(\left[X_{1}, Y_{1}\right]\right) \cap \mu\left(\left[X_{2}, Y_{2}\right]\right)=\left(\mu_{1}\left(X_{1}\right) \cap \mu_{2}\left(Y_{1}\right)\right) \cap\left(\mu_{1}\left(X_{2}\right) \cap \mu_{2}\left(Y_{2}\right)\right) \\
& =\left(\mu_{1}\left(X_{1}\right) \cap \mu_{1}\left(X_{2}\right)\right) \cap\left(\mu_{2}\left(Y_{1}\right) \cap \mu_{2}\left(Y_{2}\right)\right)=\emptyset
\end{aligned}
$$

for $\left[X_{1}, y_{1}\right] \neq\left[X_{2}, Y_{2}\right]$.

Finally, let $p^{\prime}: c \rightarrow z_{1} \ldots z_{n}$ be a production in $p^{\prime}$, then by assumption there are productions

and

$$
p_{1}: A \rightarrow x_{1} \ldots x_{n} \text { in } P_{1}
$$

$$
p_{2}: B \rightarrow Y_{1} \ldots Y_{n} \text { in } P_{2}
$$


such that $p^{\prime}$ is in $\mu_{1}\left(p_{1}\right) \cap \mu_{2}\left(p_{2}\right)$. Thus, $C$ is in $\mu_{1}(A) \cap \mu_{2}(B)$ and $z_{i}$ is in $\mu_{j}\left(X_{i}\right) \cap \mu_{2}\left(Y_{i}\right), 1 \leq i \leq n$. Hence

$p:[A, B] \rightarrow\left[X_{1}, Y_{1}\right] \ldots\left[X_{n}, Y_{n}\right]$ is a production in $P$ and $p^{\prime}$ is in $\mu(p)$ since $\mu([A, B])=\mu_{1}(A) \cap \mu_{2}(B)$ and $\mu\left(\left[X_{i}, Y_{i}\right]\right)=\mu_{1}\left(X_{j}\right) \cap \mu_{2}\left(Y_{i}\right)$, $i \leq i \leq n$. Therefore $G \stackrel{\Delta}{\Delta} G(\mu)$.

We are now in a position to prove that $\mathcal{E}_{S}$ and $\boldsymbol{E}_{S}(G)$ are distributive lattices.

\section{Theorem 3.13}

$E_{s}$ is a distributive lattice under $\leq_{s}$.

For all grammar forms $G, \boldsymbol{E}_{S}(G)$ is a distributive lattice under $\leq_{s}$.

Proof: We will only prove the first statement, the second follows by similar arguments.

We must first show that $\boldsymbol{E}_{s}$ is a lattice. Since $\mathcal{E}_{s}$ is partially ordered under $\leq_{s}$ it suffices to show the existence of a glb and lub for every pair of equivalence classes in $\varepsilon_{s}$. Let $E_{1}$ and $E_{2}$ be two equivalence classes. Then $E_{i}=\left[G_{i}\right]_{s}$, for some grammar form $G_{i}, i=1,2$. Now by Lemma 3.12 there exists a grammar form $G$ such that $\xi_{S}(G)=\xi_{S}\left(G_{1}\right) \cap \xi_{S}\left(G_{2}\right)$. Hence $[G]=E$, say, is a lower bound of $E_{1}$ and $E_{2}$. Consider $G^{\prime}$ such that $\left[G^{\prime}\right]=E^{\prime}$ is also a lower bound of $E_{1}$ and $E_{2}$. We show that $E^{\prime} \leq_{S} E$.

Now since $E^{\prime} \leq_{S} E_{i}, i=1,2$ we have $G^{\prime} \Delta_{s} G_{i}, i=1,2$. Immediately $\xi_{S}\left(G^{\prime}\right) \subseteq \mathcal{G}_{S}\left(G_{1}\right) \cap \boldsymbol{G}_{S}\left(G_{2}\right)$, therefore $\boldsymbol{\xi}_{S}\left(G^{\prime}\right) \subseteq \mathcal{G}_{S}(G)$ and $\left[G^{\prime}\right]_{S} \leq_{S}[G]_{S}$. Hence $[G]_{S}$ is the $g l b$ of $\left[G_{1}\right]$ and $\left[G_{2}\right]$.

Consider the least upper bound. Let $G_{1}=\left(V_{1}, \Sigma_{1}, P_{1}, S_{1}\right)$ and $G_{2}=\left(V_{2}, \Sigma_{2}, P_{2}, S_{1}\right)$ where $V_{1} \cap V_{2}=\left\{S_{1}\right\}$. We may assume $G_{1}$ and $G_{2}$ fulfill these conditions without any loss of generality. Define $G=(V, \Sigma, P, S)$ by: $V=V_{1} \cup V_{2}, \Sigma=\Sigma_{1} \cup \Sigma_{2}, P=P_{1} \cup P_{2}$ and $S=S_{1}$. clearly $G_{i} \underset{S}{\longrightarrow}, i=1,2$. Therefore $\left[G_{i}\right]_{S} \leq_{S}[G]_{S}, i=1,2$, and $[G]_{S}$ is an upper bound. Consider a grammar form $G^{\prime}=\left(V^{\prime}, \Sigma^{\prime}, P^{\prime}, S^{\prime}\right)$ such that $\left[G^{\prime}\right]_{S}$ is an upper bound of $\left[G_{1}\right]_{S}$ and $\left[G_{2}\right]_{S}$. We show $[G]_{S} \leq_{S}\left[G^{\prime}\right]_{S}$. Now there exist substitutions $\mu_{1}$ and $\mu_{2}$ such that $G_{i} \underset{s}{a} G^{\prime}\left(\mu_{i}\right), i=1,2$. Define a new substitution $\mu$ by: for al1 $X^{\prime}$ in $V^{\prime}, \mu\left(X^{\prime}\right)=\mu_{1}\left(X^{\prime}\right) \cup \mu_{2}\left(X^{\prime}\right)$. We have $S_{1}$ in $\mu\left(S^{\prime}\right)$ and for all $X^{\prime}, Y^{\prime}$ in $V^{\prime}, X^{\prime} \neq Y^{\prime}$ implies 


$$
\begin{aligned}
& \left.\mu\left(X^{\prime}\right) \cap \mu^{\prime}\right)=\left(Y_{1}\left(X^{\prime}\right) \cup \mu_{2}\left(X^{\prime}\right)\right) \cap\left(\mu_{1}\left(Y^{\prime}\right) \cup \mu_{2}\left(Y^{\prime}\right)\right) \\
= & \left(\mu_{1}\left(X^{\prime}\right) \cap \mu_{1}\left(Y^{\prime}\right)\right) \cup\left(\mu_{1}\left(X^{\prime}\right) \cap \mu_{2}\left(Y^{\prime}\right)\right) \cup\left(\mu_{2}\left(X^{\prime}\right) \cap \mu_{1}\left(Y^{\prime}\right)\right) \\
& u\left(\mu_{2}\left(X^{\prime}\right) \cap \mu_{2}\left(Y^{\prime}\right)\right) \\
= & \left(\mu_{1}\left(X^{\prime}\right) \cap \mu_{2}\left(Y^{\prime}\right)\right) \cup\left(\mu_{2}\left(X^{\prime}\right) \cap \mu_{1}\left(Y^{\prime}\right)\right), \\
& \text { since } \mu_{1}\left(X^{\prime}\right) \cap \mu_{i}\left(Y^{\prime}\right)=\emptyset \text { when } X^{\prime} \neq Y^{\prime} \\
= & \emptyset \text { since } V_{1} \cap V_{2}=\left\{S_{1}\right\} \text { and } X^{\prime} \neq Y^{\prime} .
\end{aligned}
$$

Finaliy $P=P_{1} \cup P_{2} \subseteq \mu_{1}\left(P^{\prime}\right) \cup \mu_{2}\left(P^{\prime}\right) \subseteq \mu\left(P^{\prime}\right)$, thus $G \underset{s}{\nabla} G^{\prime}(\mu)$ and therefore $[G]_{S} \leq_{S}\left[G^{\prime}\right]_{S}$ and $[G]_{S}$ is the $1 \mathrm{ub}$ of $\left[G_{1}\right]_{S}$ and $\left[G_{2}\right]_{S}$.

consequently, $\mathcal{E}_{S}$ is lattice under $s_{s}$. It remains to show that it is distributive.

Let $G_{i}=\left(V_{i}, \Sigma_{i}, P_{i}, S_{1}\right), i=1,2,3$ be three grammar forms with a common sentence symbol and apart from $S_{1}$, pairwise disjoint alphabets. There is again no loss of generality in this assumption since we are interested in $\left[G_{j}\right]_{S}$. Define $G=(V, \Sigma, P, S)$ by:

$$
\begin{aligned}
& V=\left(V_{1}-\Sigma_{1}\right) \times\left(\left(V_{2}-\Sigma_{2}\right) \cup\left(V_{3}-\Sigma_{3}\right)\right) \cup\left(\Sigma_{1} \times\left(\Sigma_{2} \cup \Sigma_{3}\right)\right) \\
& \Sigma=\Sigma_{1} \times\left(\Sigma_{2} \cup \Sigma_{3}\right), \\
& S=\left[S_{1}, S_{1}\right], \text { and } P \text { is defined by: }
\end{aligned}
$$

for each production $A \rightarrow X_{1} \ldots X_{n}$ of $P_{1}$

and each production $B \rightarrow Y_{1} \ldots Y_{n}$ of $P_{2} \cup P_{3}$ with $\left[X_{i}, Y_{i}\right]$ in $V$ take the production $[A, B] \rightarrow\left[X_{1}, Y_{1}\right] \ldots\left[X_{n}, Y_{n}\right]$ into $P$.

It is readily proved that $[G]_{S}=\left[G_{1}\right]_{S} \wedge\left(\left[G_{2}\right]_{S} \vee\left[G_{3}\right]_{S}\right)$ and $[G]_{S}=\left(\left[G_{1}\right]_{S} \wedge\left[G_{2}\right]_{S}\right) \vee\left(\left[G_{1}\right]_{S} \wedge\left[G_{3}\right]_{S}\right)$. Therefore $\mathscr{E}_{s}$ is distributive.

\section{Corozzary 3.14}

For arbitrary grammar forms $G_{1}$ and $G_{2}: \quad\left[G_{1}\right]_{S} \wedge\left[G_{2}\right]_{S}=[G]_{S}$, where $G$ is such that $\xi_{s}(G)=\xi_{s}\left(G_{1}\right) n \xi_{s}\left(G_{2}\right)$.

However the corresponding implication for $v$ is not true in general. More precisely if $G_{1}, G_{2}$ and $G$ are such that $\left[G_{1}\right]_{S} \vee\left[G_{2}\right]_{S}=[G]_{S}$ then the strongest implication is that $\xi_{5}\left(G_{1}\right) \cup \xi_{s}\left(G_{2}\right) \subseteq \xi_{s}(G)$. In fact equality is only obtained when either $G_{1} \underset{s}{a} G_{2}$ or $G_{2} \underset{s}{a} G_{1}$. Consider the simple example $G_{1}: S \rightarrow a, G_{2}: S \rightarrow$ a a and $G: S \rightarrow a: S \rightarrow a a$, then $[G]_{S}=\left[G_{1}\right]_{S} \vee\left[G_{2}\right]_{S}$ but $G$ is not an interpretation of either $G_{1}$ or $G_{2}$, hence

$\xi_{s}(G) \neq \xi_{s}\left(G_{1}\right) \cup \xi_{s}\left(G_{2}\right)$. observe that when $G_{1} \underset{s}{A} G_{2}$ we obtain $\left[G_{1}\right]_{s} \vee\left[G_{2}\right]_{s}=\left[G_{2}\right]_{s}$, as expected.

We are now in a position to study minimality, which we do in the following section. 


\section{II.3.4 Minimal Grammar Forms}

With the notion of strong form equivalence in mind we can ask for a given grammar form $G$ whether $G$ is the "smallest" grammar form which gives rise to $\xi_{x}(6)$, for some $x$-interpretation. In this section we demonstrate that there is an essentially unique smallest grammar form for each grammar family, modulo strong $x$-form equivalence. This question may, of course be asked for form equivalence as well. Is there a "smallest" grammar form generating each gramatical family? For example, $S \rightarrow$ a generating the finite sets and $S \rightarrow \lambda \mid a S$ generating the regular sets, are surely minimal. However apart from these simple cases little seems to be known.

Minimality modulo strong form equivalence is therefore of interest and, more so, since the construction of the minimal form of a given form is effective.

\section{Definition}

A grammar form $G$ is (production) minimal if there is no grammar form $F$ such that $F \equiv S G$ and $F$ has fewer productions than $G$. Similarly $G$ is symbol minimal if there is no grammar form $F$ such that $F \equiv_{S} G$ and $F$ has fewer symbols than $G$ (by fewer we mean $\# V_{F}<\# V_{G}$ ). We say $G$ is symbol tight if each symbol apart from the sentence symbol appears in a production of $G$.

We first have:

\section{Theorem 3.15}

For every grammar form $G=(V, \Sigma, P, S)$ there exists a production minimal grammar form $G^{\prime}=\left(V, \Sigma, P^{\prime}, S\right)$ strongly $s$-form equivalent to $G$ (strongly $g-$ form equivalent to $G$ ) such that $P^{\prime} \subseteq P$.

Proof: Clearly there exists at least one minimal grammar form $G^{\prime \prime}=\left(V^{\prime \prime}, \Sigma^{\prime \prime}, P^{\prime \prime}, S^{\prime \prime}\right)$ with $G^{\prime \prime} \equiv_{S} G$. Now $G^{\prime \prime}$ is an s-interpretation of $G$ modulo some $\mu$, therefore for each production $p^{\prime \prime}$ in $p "$ Jet $p$ be a particular production in $P$ such that $p^{\prime \prime}$ is in $\mu(p)$ and $7 e t p^{\prime}$ be the set of all such productions $p$. Consider the grammar form $G^{\prime}$. $G^{\prime}$ is a subgrammar of $G$, hence $G^{\prime} \underset{s}{\Delta} G$. However by construction $G^{\prime \prime} \Delta_{S} G^{\prime}$ and by assumption $G \underset{S}{\Delta} G^{\prime \prime}$, thus $G^{\prime} \equiv G_{S} G^{\prime}$, and because $G^{\prime \prime}$ is minimal and $G^{\prime}$ contains no more productions then $G^{\prime \prime}, G^{\prime}$ is minimal. The case of strong g-form equivalence is proved similarly. 
We now obtain the main theorem for s-interpretations.

\section{Theorem 3.16}

Let $G=(V, \Sigma, P, S)$ be a grammar form. For every two grammar forms $F_{1}$ and $F_{2}$ which are s-interpretations of $G$ and are production minimal, symbol tight and strongly $s$-form equivalent to $G, F_{1}$ and $F_{2}$ are isomorphic.

Proof: Let $F_{i}=\left(V_{i}, \Sigma_{i}, P_{i}, s_{i}\right), i=1,2$ be two arbitrary production minimal, symbol tight, strongly $s$-form equivalent grammar forms of $G$. clearly $F_{1} \equiv F_{2}$. Therefore there exists $\mu_{2}$ such that $F_{1} \underset{s}{\&} F_{2}\left(\mu_{2}\right)$. clearly $P_{1} \subseteq H_{2}\left(P_{2}\right)$. Since $F_{1}$ is symbol tight every symbol $x_{1}$ in $V_{1}$ apart from $S_{1}$ appears in some production in $P_{1}$, that is, $X_{1}$ is the image of some $x_{2}$ in $V_{2}$ under $\mu_{2}$, for all $x_{1}$ in $V_{1}$. Now observe that $\mu_{2}(X) \neq \emptyset$, for all $x$ in $V_{2}$. Otherwise all productions involving $X$ could be removed from $P_{2}$ yielding a grammar form strongly s-form equivalent to $F_{1}$ but having fewer productions, a contradiction.

since $\mu_{2}(X) \cap \mu_{2}(Y)=$ for all $X \neq Y$ in $V_{2}$ we have $\# V_{2} \leq \# V_{1}$. Similarly there exists $\mu_{1}$, such that $F_{2} \rightarrow F_{1}\left(\mu_{2}\right)$. By similar arguments $\# V_{1} \leq \# V_{2}$. Hence $\# V_{1}=\# V_{2}$. Therefore $\mu_{2}$ is an isomorphism and $\mu_{1}=\mu_{2}^{-1}$.

We now extend this result to g-interpretations. In this case, essentially unique is pseudo-isomorphism, rather than isomorphism.

\section{Definition}

Let $G_{i}=\left(V_{i}, \Sigma_{i}, P_{i}, S_{i}\right) i=1,2$ be two gramar forms. Then $G_{1}$ and $G_{2}$ are pseudo-isomorphic with respect to $\mu_{1}$ and $\mu_{2}$, where $G_{1} \overleftrightarrow{g} G_{2}\left(\mu_{2}\right)$ and $G_{2} \overleftrightarrow{g} G_{1}\left(\mu_{1}\right)$, if:

(i) $\mu_{1}: v_{1}-\Sigma_{1} \rightarrow V_{2}-\Sigma_{2}, \mu_{2}: V_{2}-\Sigma_{2} \rightarrow V_{1}-\Sigma_{1}$ are surjections and $\mu_{2}=\mu_{1}^{-1}$ on this restricted domain, and

(ii) $\bar{\mu}_{1}: P_{1} \rightarrow P_{2}$ defined by $\bar{\mu}_{1}(p)=\mu_{1}(p) \cap P_{2}$ for all $p$ in $P_{1}$, and $\bar{\mu}_{2}$ defined analogousiy are surjections. Further $\bar{\mu}_{2}=\bar{\mu}_{1}^{-1}$.

We say $G_{3}$ and $G_{2}$ are pseudo-isomorphic if there are substitutions $\mu_{1}$ and $\mu_{2}$ such that $G_{1}$ and $G_{2}$ are pseudo-isomorphic with respect to $\mu_{1}$ and $\mu_{2}$.

Let $G_{i}=\left(V_{i}, \Sigma_{i}, P_{i}, S_{i}\right), i=1,2$ be two grammar forms with $G_{2} \vec{g} G_{1}\left(\mu_{1}\right)$ and $\bar{\mu}_{1}$ defined as above. We say $\bar{\mu}_{1}$ is terminal word preserving if: 
for each production $p: A \rightarrow x_{0} A_{1} \cdots A_{n} x_{n}$ in $P_{1}(n=0$ is interpreted as $\left.A \rightarrow x_{0}\right) \bar{\mu}_{1}(p)$ has the form $B \rightarrow y_{0} B_{1} \cdots B_{n} y_{n}$ where $x_{i}=\lambda$ iff $y_{i}=\lambda, 0 \leq i \leq n$.

Condition ( $i$ ) in the definition means that the nonterminals of $G_{1}$ and $G_{2}$ are isomprphic. Condition ( $i j$ ) on the other hand implies that $P_{1}$ and $P_{2}$ are almost isomorphic. When condition (i) holds for both terminals and nonterminals of $G_{1}$ and $G_{2}$, condition (ii) is clearly satisfied as in Theorem 3.16 for s-interpretations. Let us consider two examples of pseudo-isomorphism.

\section{Example 3.1}

Let $G_{1}$ and $G_{2}$ be defined by the productions:

$G_{7}: A \rightarrow a b A a a ; A \rightarrow a$

$G_{2}: S \rightarrow a S b ; S \rightarrow a b a$

Clearly $G_{1} \underset{g}{\Delta} G_{2}\left(\mu_{2}\right)$ and $G_{2} \underset{g}{\leftrightarrow} G_{1}\left(\mu_{\eta}\right)$ where:

$\mu_{1}(A)=S ; \mu_{1}(a)=\{\lambda, a, b, a b a\}$ and $\mu_{j}(b)=\{\lambda\} ;$

$\mu_{2}(S)=A ; \mu_{2}(a)=\{\lambda, a b\}$ and $\mu_{2}^{\prime}(b)=\{a, a a\}$.

Hence condition ( $i$ ) is satisfied. Further

$$
\begin{aligned}
& \bar{\mu}_{1}(A \rightarrow a b A a a)=S \rightarrow a S b ; \bar{\mu}_{1}(A \rightarrow a)=S \rightarrow a b a ; \text { and } \\
& \bar{\mu}_{2}(S \rightarrow a S b)=A \rightarrow a b A a a ; \bar{\mu}_{2}(S \rightarrow a b a)=A \rightarrow a
\end{aligned}
$$

therefore $\bar{\mu}_{1}$ and $\bar{\mu}_{2}$ fulfill condition ( $\left.i i\right)$ and $G_{1}$ and $G_{2}$ are pseudoisomorphic. Note that they are not isomorphic.

\section{Example 3.2}

Let $G_{1}$ and $G_{2}$ be defined by the productions:

$G_{1}: S \rightarrow a S ; S \rightarrow b S ;$ and

$G_{2}: S \rightarrow a b S ; S \rightarrow b a S$

Then letting $\mu_{1}(a)=\{a b\}, \mu_{1}(b)=\{b a\}$ and $\mu_{1}(S)=S, G_{2} \underset{g}{\Delta_{1}}\left(G_{1}\right)$ and letting $\mu_{2}(a)=\{a, b\}, \mu_{2}(b)=\{\lambda\}$ and $\mu_{2}(s)=s$ we have $\mathrm{G}_{1} \rightarrow \mathrm{G}_{2}\left(\mu_{2}\right)$. However $\bar{\mu}_{1}(S \rightarrow \mathrm{aS})=S \rightarrow \mathrm{abS} ; \bar{\mu}_{1}(S \rightarrow b S)=S \rightarrow b a S$; but $\bar{\mu}_{2}(S \rightarrow a b S)=\{S \rightarrow a S, S \rightarrow b S\}$ and $\bar{\mu}_{2}(S \rightarrow b a S)=\{S \rightarrow a S ; S \rightarrow b S\}$ hence $\bar{\mu}_{2}$ is not $1: 1$ onto and condition ( $i i$ ) does not hold. Moreover there is no $\mu_{2}$ such that $\bar{\mu}_{2}$ fulfills condition (ii) since $\mu_{2}(a b)=\mu_{2}(a) \mu_{2}(b)=\mu_{2}(b) \mu_{2}(a)=\mu_{2}(b a)$ in this situation. Before turning to the main theorem of this section, we need the following straightforward result.

Lemma 3.17

Let $G_{i}=\left(V_{i}, \Sigma_{i}, P_{i}, S_{i}\right), 1=1,2$ be two pseudo-isomorphic grammar forms with respect to $\mu_{1}$ and $\mu_{2}$, then $\bar{\mu}_{1}$ and $\bar{\mu}_{2}$ are terminal word preserving. 
Proof: Since $G_{1}$ and $G_{2}$ are pseudo-isomorphic with respect to $\mu_{1}$ and $\mu_{2}$, then $\bar{\mu}_{1}^{-1}=\bar{\mu}_{2}$. If $\bar{\mu}_{1}$ is not terminal word preserving, then there is a production:

$$
p: A_{0} \rightarrow x_{0} A_{1} \cdots A_{n} x_{n} \text { in } P_{1}
$$

and its corresponding production

$$
q: B_{0} \rightarrow y_{0} B_{1} \cdots B_{n} y_{n} \text { in } P_{2}
$$

such that $\bar{\mu}_{j}(p)=q$ and there is an $x_{i} \neq \lambda, y_{i}=\lambda$ for some $i, 0 \leq i \leq n$. In which case $\bar{\mu}_{1}^{-1}(q)=\bar{\mu}_{2}(q) \neq p$, since for any substitution $\mu, \mu(\lambda)$ $=\{\lambda\}$. This is a contradiction, therefore $\bar{\mu}_{1}$ is terminal word preserving. Similarly $\bar{\mu}_{2}$ is terminal word preserving.

We now have:

\section{Theorem 3.18}

Let $G_{1}$ and $G_{2}$ be two symbol tight, minimal, strong g-form equivalent grammar forms with $G_{2} \underset{g}{\Delta} G_{1}\left(\mu_{1}\right)$ and $G_{1} \underset{g}{\Delta} G_{2}\left(\mu_{2}\right)$. Then $G_{1}$ and $G_{2}$ are pseudo-isomorphic with respect to $\mu_{1}$ and $\mu_{2}$.

Proof: That condition ( $i$ ) of pseudo-isomorphism holds follows in a similar manner to the proof of Theorem 3.16, except on 1 y nonterminals are considered. It remains to show condition (ij) holds. Let $G_{i}=\left(V_{i}, \Sigma_{i}, P_{i}, S_{i}\right), i=1,2$. Define $\bar{\mu}_{1}: P_{1} \rightarrow 2^{P} 2$ and $\bar{\mu}_{2}: P_{2} \rightarrow 2^{P_{1}}$ by: $\bar{\mu}_{1}(p)=\mu_{1}(p) \cap P_{2}$ for all $p$ in $P_{1}$, and $\bar{\mu}_{2}(p)=\mu_{2}(p) \cap P_{1}$ for all $p$ in $P_{2}$. Now $\sum_{p \text { in } p} \bar{\mu}_{1}(p)=P_{2}$ and $\underbrace{}_{p \text { in } P_{2}} \bar{\mu}_{2}(p)=P_{1}$, since $G_{2} \underset{g}{\Delta} G_{1}\left(\mu_{1}\right)$ and $G_{1} \underset{g}{\Delta} G_{2}\left(\mu_{2}\right)$. Suppose $\vec{\mu}_{1}(p)=\emptyset$ for some $p$ in $P_{1}$. Consider $G_{p}=\left(V_{1}, \Sigma_{1}, P_{1}-\{p\}, S_{1}\right)$. Now $G_{p} \underset{g}{\rightarrow} G_{1}, G_{2} \vec{g} G_{p}\left(\mu_{1}\right)$ and $G_{1} \vec{g} G_{2}\left(\mu_{2}\right)$ hence $G_{p}$ is strangly $g$-form equivalent to $G_{1}$ and has fewer productions than $G_{1}$, a contradiction. Hence $\bar{\mu}_{1}(p) \neq \emptyset$ for each $p$ in $P_{1}$. It now suffices to show that $\# \bar{\mu}_{1}(p)=1$ for each $p$ in $P_{1}$. Number the productions of $p_{1}$ as $p=p_{1}, \ldots, p_{n}$. Define subsets $M_{1}, M_{2}, \ldots$ of $P_{2}$ recursively by:

$$
\begin{aligned}
& M_{1}=\bar{\mu}\left(p_{1}\right) \text { and } \\
& M_{i+1}=M_{i} \cup \bar{\mu}_{1}\left(p_{i+1}\right), \text { for } i \geq 1 .
\end{aligned}
$$

Since $\# P_{1}=\# P_{2}, P_{2}=\bigsqcup_{j=1}^{n} \bar{\mu}_{1}\left(p_{j}\right), M_{j} \subseteq M_{j+1}$ for each $j<n$, and $\# M_{1} \geq 2$ it follows that $M_{i+1}=M_{j}$ for some $i<n$. Hence $G_{p_{i+1}}=\left(V_{1}, \Sigma_{1}, P_{1}-\left\{p_{i+1}\right\}, S_{1}\right)$ is also production minimal; a contradiction. 
Therefore $\bar{\mu}_{1}$ is surjective. Analogously $\bar{\mu}_{2}$ is surjective. It remains to show that $\bar{\mu}_{2}=\bar{\mu}_{1}^{-1}$.

Consider $\bar{\mu}_{1} \bar{\mu}_{2}$ defined by $\bar{\mu}_{1} \bar{\mu}_{2}(p)=\bar{\mu}_{1}\left(\bar{\mu}_{2}(p)\right)$ for all $p$ in $p_{2}$. It suffices to prove $\bar{\mu}_{1} \bar{\mu}_{2}$ is the identity map on $P_{2}$. Assume otherwise, that is there exists $p$ in $P_{2}$ such that $\bar{\mu}_{1} \bar{\mu}_{2}(p)=q$ and $p \neq q$. Now for nonterminals $\mu_{1}$ and $\mu_{2}$ are surjective and $\mu_{2}=\mu_{1}^{-1}$, therefore letting $p$ be $A_{0} \rightarrow x_{1} A_{1} \cdots A_{n} x_{n}=n \geq 0$ then $q$ is $A_{0} \rightarrow y_{0} A_{1} \cdots A_{n} y_{n}$ for some $y_{i}, 0 \leq i \leq n$. Moreover $q$ is in $\mu_{1}\left(\mu_{2}(p)\right)$ therefore $y_{i} \neq \lambda$ implies $x_{i} \neq \lambda, 0 \leq i \leq n$. Consider $G_{q}=\left(V_{2}, \Sigma_{2}, p_{2}-\{q\}, s_{2}\right)$. As above $G_{q}$ can be shown to be production minimat and strongly $g$-form equivalent to $G_{2}$, giving a contradiction. 


\section{II.4 Normal Form, Closure and Characterization Results}

Normal forms have always been a central topic in the study of grammars and their languages. Grammar forms not only enable the notion of normal form to be made rigorous but also allow its generalization in a natural way. Let $G$ be a grammar form for which $\mathcal{L}_{S}(G, \Rightarrow)=\mathcal{L}(C F)$, then any grammar $F$ is said to be in $G$-normal form if $F \underset{S}{a} G$. We consider a restricted version of this notion in which $G$ is a twosymbol form. We are able to prove a super-normal form theorem for context-free grammars and grammar forms, which includes Chomsky Normal Form and Greibach two-standard form as special cases. This we do in Section 4.1 , where we also derive other reduction results.

In section 4.2 we demonstrate that $\mathcal{L}_{\mathrm{g}}(G, \Rightarrow)$ is a fult semi-AFL for every infinite $G$ and demonstrate that $\mathcal{L}_{S}(G, \Rightarrow)$ is in the worst case only closed under intersection with regular sets and dfisubstitutions. Some further specialized closure properties are to be found in Section 6.1 .

Finally in Section 4.3 three results are proved: for every infinite $G, \mathcal{L}_{g}(G, \Rightarrow)$ is a full principal semi-AFL, every g-grammatical family apart from the finite sets is $s$-grammatical and we characterize those grammar forms $G$ for which $\mathcal{L}_{\mathrm{S}}(G, \Rightarrow)=\mathcal{L}(R E G)$ or $\mathcal{L}(L I N)$.

\section{II.4.1 Reduction Results}

In order to prove that each g-grammatical family is a full principal semi-AFL, it is convenient to first derive some specific normal form theorems for grammar forms under both $\mathrm{g}$ - and $\mathrm{s}$-interpretations. Most of these, which are of interest in their own right, are stated only for s-interpretations since if $\mathcal{L}_{S}\left(G_{1}, \Rightarrow\right)=\mathcal{L}_{s}\left(G_{2}, \Rightarrow\right)$ then by Corollary $1.2, \mathcal{Z}_{\mathrm{g}}\left(G_{1}, \Rightarrow\right)=\mathcal{L}_{\mathrm{g}}\left(G_{2}, \Rightarrow\right)$ that is $s$-form equivalence implies $g$-form equivalence. Our first result is stated without proof.

\section{Proposition 4.1}

For every grammar form $G$ there exists an s-form equivalent grammar form $G_{1}$ which is reduced.

Simply discard from $G$ the useless terminals, nonterminals and productions to give the reduced grammar form $G_{1}$.

Recall that a single production is a production of type $A \rightarrow B$. We say a grammar is single-free if it has no single productions. 


\section{Theorem 4.2}

For every grammar form $G=(V, \Sigma, P, S)$ there exists a single-free $s-$ form equivalent grammar form $G_{1}=\left(V, \Sigma, P_{1}, S\right)$.

Proof: We carry out the standard construction to remove single productions. Without $105 s$ of generality assume $G$ is reduced. For each $A$ in $V-\Sigma$, let $s(A)=\left\{B: A \Rightarrow B\right.$ by way of single productions ${ }^{+}$. Clearly $G$ is single-free if for all $A$ in $V-\Sigma, s(A)=\emptyset$. In this case $G_{1}$ is identified with $G$. Otherwise construct $G_{2}=\left(V, \Sigma, P{ }_{2}, S\right)$ where $P_{2}=P \cup\{A \rightarrow B: B$ is in $s(A)$ and $B \rightarrow B$ is in $P\}$. By corollary 2.10, $\mathscr{Z}_{s}\left(G_{2} \Rightarrow\right)=\mathcal{L}_{s}(G, \Rightarrow)$. Now letting $P_{1}=P_{2}-(V \times V), \mathcal{Z}_{s}\left(G_{1}, \Rightarrow\right) \subseteq \mathcal{Z}_{s}\left(G_{2}, \Rightarrow\right)$. It remains to show that $\mathcal{L}_{\mathrm{S}}\left(G_{2}, \Rightarrow\right) \subseteq \mathscr{Z}_{\mathrm{S}}\left(G_{1}, \Rightarrow\right)$.

Consider $G_{2}^{\prime}=\left(V^{\prime}, \Sigma^{1}, P_{2}^{\prime}, S^{\prime}\right) \bigotimes_{S}^{S} G_{2}(\mu)$. It suffices to exhibit an s-interpretation $G_{j}^{\prime}$ of $G_{1}$ with $L\left(G_{j}^{\prime} \Rightarrow\right)=L\left(G_{2}^{\prime} \Rightarrow\right)$. We can assume that for all $A^{\prime}, B^{\prime}$ in $V^{\prime}-\Sigma^{\prime}$ with $B^{\prime}$ in $s\left(A^{\prime}\right)$ that $A^{\prime} \rightarrow B^{\prime}$ is in $P_{2}^{\prime}$ for all $B^{\prime}$ such that $B^{\prime} \rightarrow B^{\prime}$ is in $P_{2}^{\prime}$. This follows by the definition of $G_{2}$ and by Corollary 2.10 for grammars rather than grammar forms. construct $G_{j}^{\prime}=\left(V^{\prime}, \Sigma^{\prime}, P_{j}^{\prime}, S^{\prime}\right)$ by taking $P_{j}^{\prime}=P_{2}^{\prime}-\left(V^{\prime} \times V^{\prime}\right)$. That $L\left(G_{j}^{\prime}, \Rightarrow\right)=L\left(G_{2}^{\prime} \Rightarrow\right)$ is a standard result.

Recall that a grammar (form) is $\lambda$-free if no production in the grammar has the empty word on its right hand side.

\section{Theorem 4.3}

Let $G=(V, \Sigma, P, S)$ be a nontrivial grammar form. Then there exists a grammar form $H$ such that $H$ is $\lambda$-free and $H$ is $s$-form equivalent to $G$.

Proof: Since $G$ is nontrivial $L(G, \Rightarrow)$ contains at least one non-empty word. This enables the standard construction to be carried out.

First define a substitution $\tau$ on $V *$ by:

$$
\begin{aligned}
\tau(a)= & a, \text { for all a in } \Sigma, \\
\tau(A)= & \text { either }\{A, \lambda\} \text { if } A \Rightarrow^{+} \lambda \text { in } G \\
& \text { or }\{A\} \text { otherwise. }
\end{aligned}
$$

Consider $F=\left(V, \Sigma, P_{F}, S\right)$ defined by:

$$
P_{F}=\{A \rightarrow B: A \rightarrow \alpha \text { is in } P \text { and } B \text { is in } T(\alpha)\} \text {. }
$$

By Corollary $2.10 \mathcal{Z}_{S}(F, \Rightarrow)=\mathcal{Z}_{S}(G, \Rightarrow)$.

Second, consider $H=\left(V, \Sigma, P_{H}, S\right)$ defined by:

$$
P_{H}=P_{F}-(V \times\{\lambda\}) \text {. }
$$

since $H$ is a subgrammar of $F$ it is clear that $\mathscr{L}_{S}(H, \Rightarrow) \subseteq \mathscr{L}_{s}(F, \Rightarrow)$. 
We now show that $\mathscr{L}_{S}(G, \Rightarrow) \subseteq \mathcal{L}_{S}(H, \Rightarrow)$ completing the proof. Consider an arbitrary interpretation $G^{\prime} \prod_{S} G(\mu)$, where $G^{\prime}=\left(V^{\prime}, \Sigma^{\prime}, P^{\prime}, S^{\prime}\right)$.

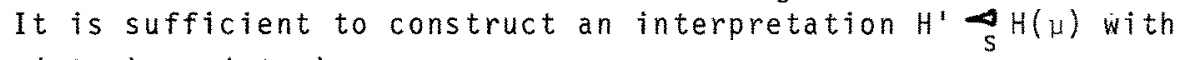
$L\left(H^{\prime}, \Rightarrow\right)=L\left(G^{\prime}, \Rightarrow\right)$.

However the construction detailed above to obtain $H$ from $G$ is the standard $\lambda$-removal construction. Since, under s-interpretation, $A^{\prime} \Rightarrow^{+} \lambda$ in $G^{\prime}$ only if $\mu^{-1}\left(A^{\prime}\right) \Rightarrow^{+} \lambda$ in $G$, then we can define $\tau^{\prime}$, giving $F^{\prime} \underset{S}{\Delta} F$ with $L\left(F^{\prime}, \Rightarrow\right)=L\left(G^{\prime}, \Rightarrow\right)$. Now $H^{\prime}{\underset{S}{S}}_{H} H$, since $P_{H^{\prime}}=P_{F^{\prime}}-\left(V^{\prime} \times\{\lambda\}\right)$ and $L\left(H^{\prime}, \Rightarrow\right)=L\left(G^{\prime}, \Rightarrow\right)$ by standard techniques.

We say that $G=(V, \Sigma, P, S)$ is c-reduced if it is:

(i) reduced,

(ii) $\lambda$-free,

( $i i i)$ single-free, and

(iv) for all $A$ in $V-\Sigma, A \neq S$, there exists a production $A \rightarrow x A y$ in $P$ such that $x y \neq \lambda$.

\section{$\underline{\text { Lemma } 4.4}$}

Each nontrivial grammar form $G=(V, \Sigma, P, S)$ has an $s-$ form equivalent c-reduced grammar form $\mathrm{H}$.

Proof: We may assume $G$ is reduced, $\lambda$-free and single-free. $A$ in $V-\Sigma$ is said to be partially self-embedding in $G$, if either $A=S$ or there exists $x$ and $y, x y \neq \lambda$ such that $A \Rightarrow^{+} x A y$.

Assume each nonterminal $A \neq S$ is partially self-embedding, then there is a derivation $A \Rightarrow^{+} x A y$ with $x y \neq \lambda$ and by Corollary 2.10 we can assume $A \rightarrow x A y$ is in $P$ without any loss of generality. In this case take $H$ to be equal to $G$.

otherwise suppose that there are $k>0$ nonterminals which are non-partially self-embedding. Carry out an iterative construction based on the number of non-partially self-embedding nonterminals.

Consider $G_{1}=\left(V_{l}, \Sigma, P_{1}, S\right)$ defined as follows:

$V_{1}=V-\{A\}$, where $A$ is a non-partially self-embedding nonterminal, and

$$
\begin{aligned}
P_{1}= & \{B \rightarrow \bar{B}: B \rightarrow B \text { is in } P, B \neq A \text {, and } \bar{B} \text { is obtained from } \\
& B \text { by replacing each occurrence of } A \text { in } B \text { by some } \\
& \text { word in } r(A)\},
\end{aligned}
$$

where $r(A)=\{\alpha: A \rightarrow \alpha$ is in $P\}$.

Clearly $G$ simulates $G_{1}$, therefore $\mathcal{L}_{s}\left(G_{1}, \Rightarrow\right) \subseteq \mathcal{L}_{s}(G, \Rightarrow)$. That $\mathcal{L}_{s}(G, \Rightarrow) \subseteq \mathcal{L}_{s}\left(G_{1} \Rightarrow\right)$ follows from the back-substitution lemma, Lemma 2.12 . Note that $G_{1}$ is reduced if $G$ is reduced, $G_{1}$ is $\lambda$-free and $G_{1}$ is single-
free. 
Further, $G_{1}$ has k-1 non-partially self-embedding nonterminals. Therefore, iterate the construction $k$ times to obtain $G_{k}$ all of whose nonterminals are partially self-embedding. Let $H$ be $G_{k}$ to complete the lemma.

A grammar $G=(V, \Sigma, P, S)$ is sequential if its nonterminals can be numbered $S=A_{1}, \ldots, A_{n}$, so that $A_{j} \rightarrow \alpha A_{j} B$ belongs to $P$ implies $i \leq j$.

We say $G$ is s-reduced if it both c-reduced and sequential.

It is well known that not al1 context-free languages are sequential (that is, can be generated by a sequential grammar). The language generated by the grammar $G$, defined by the productions:

$$
\begin{aligned}
& S \rightarrow a A a ; S \rightarrow \lambda \\
& A \rightarrow a A a ; A \rightarrow b B b \\
& B \rightarrow a B a ; B \rightarrow b S b
\end{aligned}
$$

is an example of such a non-sequential language.

Under the two interpretation mechanisms we are discussing we consider whether each g- or s-grammatical family is sequential, that is, generated by a sequential grammar form. Our first result, for s-interpretations is negative, while our second, for g-interpretations is positive.

\section{Theorem 4.5}

Let $G$ be defined by the productions:

$S \rightarrow$ baAab; $S \rightarrow$ bacadedacab

$A \rightarrow a A a ; A \rightarrow c a B a c$

$A \rightarrow a A a ; A \rightarrow c a B a c$

$B \rightarrow a B a ; B \rightarrow d S d$

Then $\mathcal{L}_{\mathrm{S}}(G, \Rightarrow)$ is not a sequential s-grammatical family.

Proof: First note that $L(G, \Rightarrow)=\left\{x e m i(x): x\right.$ is in $\left(b a^{+}\right.$ca $\left.{ }^{+} d\right){ }^{*}$ bacad $\}$. It is straightforward using a result due to shamir to demonstrate that $L(G, \Rightarrow)$ is not sequential. Second, observe that every word in every language in $\mathcal{L}_{S}(G, \Rightarrow)$ has at least 5 distinct symbols. This follows from the fact that the only terminating production $S$ - bacadedacab, contains each symbol of $L(G, \Rightarrow)$ at least once. Third, observe that if $L$ is in $\mathcal{L}_{S}(G, \Rightarrow)$ and $L \subseteq\{a, b, c, d, e\}$ * then there exists a permutation $\pi$ of the symbols $a, b, c, d$, e such that

$L \subseteq \pi(L(G, \Rightarrow))$ where $\pi(L(G, \Rightarrow))$ denotes the language obtained from $L(G, \Rightarrow)$ by applying 
the permutation $\pi$ to all its words. The validity of this observation follows by examining the productions of $G$. Since $L=L\left(G^{\prime}, \Rightarrow\right)$ for some $G^{\prime} \triangle_{s} G$, each of the symbols $a, b, c, d$, e in L must be an intefpretation of exactiy one of the symbols $a, b, c, d$, e in the alphabet of $G$. The inclusion, $L \subseteq \pi(L(G, \Rightarrow))$ now follows because derivations in $G$ ' must follow the same pattern as those according to $G$, that is an interpretation of $S$ yields that of $A$, which, in turn, yields that of $B$, after which we return to an interpretation of $S$.

We now establish the Theorem by contradiction. Assume there is a grammar form $F$, which is sequential and $s$-form equivalent to $G$. There is an $F^{\prime} \Delta_{S} F(\mu)$ such that $F^{\prime}$ is reduced and $L\left(F^{\prime}, \Rightarrow\right)=L(G, \Rightarrow)$. Let $F_{1}$ be the smallest subgrammar of $F$ such that $F^{\prime} \vec{s} F_{1}(\mu)$, that is every production of $F_{1}$ is used in defining some production in $F^{\prime}$. Immediately it follows that the terminal alphabet of $F_{1}$ consists of at most 5 symbols. Since each $L$ in $\mathcal{L}_{S}(G, \Rightarrow)$ has at least 5 symbols, $F_{1}$ has exactly 5 terminal symbols. Rename the terminal alphabet of $F_{1}$ without changing its generative capacity, in such a way that $\mu$ becomes the identity on $\{a, b, c, d, e\}$ and is everywhere else unchanged. Hence $L(G, \Rightarrow)=L\left(F^{\prime}, \Rightarrow\right) \subseteq L\left(F_{\eta}, \Rightarrow\right)$. Since $L \subseteq \pi(L(G, \Rightarrow))$ for a $11 L \subseteq\{a, b, c, d, e\} *$ and $L$ in $\mathcal{L}_{s}(G, \Rightarrow)$, we have $L\left(F_{1}, \Rightarrow\right)=L\left(F^{\prime}, \Rightarrow\right)$. Finally, since $F$ is sequential so is $F_{j}$, thus $L(G, \Rightarrow)$ is sequential, a contradiction.

However under g-interpretation we can obtain a sequential g-form equivalent grammar form when given an arbitrary grammar form.

Theorem 4.6

Let $G=(V, \Sigma, P, S)$ be a grammar form. There exists a $g$-form equivalent grammar form $H$ which is s-reduced.

Proof: By previous results we can assume $G$ is c-reduced. Define a relation $\approx$ on $V-\Sigma$ by:

$A \sim B$ if $A \Rightarrow{ }^{+} \alpha_{1} B B_{1}$ and $B \Rightarrow{ }^{+} \alpha_{2} A B_{2}$,

for some $\alpha_{i}, \beta_{i}, i=1,2$.

Clearly $\sim$ is an equivalence relation of finite index. Let [A] denote the equivalence class of $A$ and $X_{\text {[A] }}$ be a distinguished element of [A] with $X_{[S]}=S$. Let $\nabla=\left\{X_{[A]}: A\right.$ is in $\left.V-\Sigma\right\}$. Define a grammar form $H=\left(V_{H}, \Sigma, P_{H}, S\right)$ as follows: $V_{H}=\nabla u \Sigma$ and $P_{H}$ is obtained by replacing every nonterminal in every production of $P$ by its corresponding distinguished element.

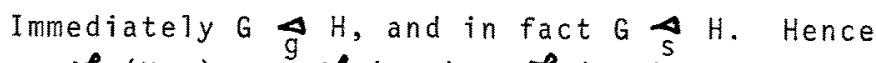
$\mathcal{L}_{g}(G, \Rightarrow) \subseteq \mathcal{L}_{g}(H, \Rightarrow)$ and $\mathcal{L}_{s}(G, \Rightarrow) \subseteq \mathcal{L}_{s}(H, \Rightarrow)$. 
Consider the converse inclusion for g-interpretations. Define a new grammar form $F=(V, \Sigma, P \cup\{A \rightarrow B: A \neq B$ and $A \sim B\}, S)$. F simu1 ates $H$, therefore $\mathcal{L}_{g}(H, \Rightarrow) \subseteq \mathcal{Z}_{g}(F, \Rightarrow)$. We need to show that $\mathcal{L}_{g}(F, \Rightarrow) \subseteq \mathcal{L}_{g}(G, \Rightarrow)$ to complete the proof.

since for each $A, B$ in $V-\sum$ with $A \neq B$ and $A \sim B$ there are derivations $A \Rightarrow{ }^{+} \alpha_{1} B \beta_{1}$ and $B \Rightarrow^{+} \alpha_{2} A \beta_{2}$ in $G$, for some $\alpha_{j}, \beta_{j}, i=1,2$, there are derivations $A \Rightarrow{ }^{+} u_{1} B v_{1}$ and $B \Rightarrow{ }^{+} u_{2} A v_{2}$ in $G$, for some $u_{i}$, $v_{i}$ in $\Sigma^{*}, i=1,2$. Hence we can assume $A \rightarrow u_{1} B v_{1}$ and $B \rightarrow u_{2} A v_{2}$ are in $P$ by Corollary 2.10. Hence $F \underset{g}{\Delta} G$ and $\mathcal{L}_{g}(F, \Rightarrow) \subseteq \mathcal{L}_{g}(G, \Rightarrow)$.

We now prepare the way for demonstrating that left recursion may be removed from a grammar form without disturbing its grammatical family. This result will then be used later to derive a super-normal form result.

Lemma 4.7

Let $G=(V, \Sigma, P, S)$ be a grammar form and $A \rightarrow \alpha_{1} B \alpha_{2}$ a production in $P$, where $B$ is a nonterminal in $V-\Sigma$. Then $G_{1}=(V, \Sigma, P,, S)$, where $P_{1}=\left(P-\left\{A \rightarrow \alpha_{1} B \alpha_{2}\right\}\right) \cup\left\{A \rightarrow \alpha_{1} \beta \alpha_{2}: B \rightarrow \beta\right.$ is in $\left.P\right\}$, is $s-$ form equivalent to $G$.

Proof: 0bserve that $G$ simulates $G_{1}$, hence we have immediately that $\mathcal{Z}_{\mathrm{S}}\left(G_{1}, \Rightarrow\right) \subseteq \mathcal{L}_{\mathrm{S}}(G, \Rightarrow)$. Conversely, given an arbitrary interpretation $G^{\prime} \vec{s} G$ it is straightforward to construct $G_{j}^{\prime} \underset{s}{\rightarrow} G_{1}$ such that $L\left(G^{\prime}, \Rightarrow\right)=L\left(G_{j}^{\prime} \Rightarrow\right)$ by the corresponding standard result for grammars. Hence the lemma follows.

We also need:

\section{Lemma 4.8}

Let $G=(V, \Sigma, P, S)$ be a $C$-reduced grammar form and $A$ a nonterminal in $V$. Let the $A$-productions in $P$ be denoted by:

$$
A \rightarrow A \alpha_{1}|\ldots| A \alpha_{r}\left|\beta_{1}\right| \ldots \mid B_{s} \text {, }
$$

where the $B_{j}$ do not begin with an $A$. Construct $G_{1}=\left(V_{1}, E, P_{1}, S\right)$

where $V_{1}=V \cup\{X\}, X$ a new nonterminal and $P_{1}$ is the same as $P$ except that the $A$-productions are replaced by the productions:

$$
\begin{aligned}
& A \rightarrow \beta_{1}|\ldots| \beta_{s}\left|\beta_{1} X\right| \ldots \mid \beta_{s} X \\
& X \rightarrow \alpha_{1}|\ldots| \alpha_{r}\left|\alpha_{1} X\right| \ldots|| \alpha_{r} X .
\end{aligned}
$$

Then $\mathscr{L}_{s}\left(G_{1}, \Rightarrow\right)=\mathscr{L}_{s}(G, \Rightarrow)$. 
Proof: First observe that we cannot use simulation techniques to prove this lemma since we have interchanged left linear productions with right linear ones.

ctaim 1: $\quad \mathcal{L}_{\mathrm{s}}(G, \Rightarrow) \subseteq \mathcal{L}_{\mathrm{s}}\left(G_{1}, \Rightarrow\right)$.

Consider an arbitrary interpretation $G^{\prime} \vec{S}_{S} G(\mu)$, where

$G^{\prime}=\left(V^{\prime}, \Sigma^{\prime}, P^{\prime}, S^{\prime}\right)$ and $\mu(A)=\left\{A_{1}, \ldots, A_{t}\right\}$ say. We may assume that

$G^{\prime}$ is reduced since $G$ is reduced. Construct a grammar $G_{j}^{\prime}=\left(V_{j}^{\prime}, \Sigma^{\prime}, P^{\prime}, S^{\prime}\right)$ where $V_{i}=V^{\prime} \cup\left\{A_{i}^{(j)}: 1 \leq i, j \leq t\right\}$ and the $A_{j}^{(j)}$ are new nonterminals. Define $P_{j}^{\prime}$ to be the same as $P^{\prime}$ except for the images of A-productions, which are replaced by the following productions:

(i) if $A_{i} \rightarrow B^{\prime}$ is in $P^{\prime}$ then take $A_{i} \rightarrow B^{\prime}$ into $P_{j}^{\prime}$,

(ii) if $A_{i} \rightarrow A_{j} \alpha^{\prime}$ is in $P^{\prime}$ then take $A_{j}^{(k)} \rightarrow \alpha^{\prime} A_{j}^{(k)}$ into $P_{j}^{\prime}$, for a $11 k, 1 \leq k \leq t$

(iii) if $A_{i} \rightarrow A_{j} \alpha^{\prime}$ is in $P^{\prime}$ then take $A_{j}^{(i)} \rightarrow \alpha^{\prime}$ into $P_{j}^{\prime}$,

(iv) if $A_{j} \rightarrow B^{\prime}$ is in $P^{\prime}$ and $A_{i} \Rightarrow A_{j}$, then take $A_{i} \rightarrow B^{\prime} A_{j}^{(i)}$ into $P i$

This is essentially the standard left linear to right linear grammar construction, modified to take into account the fact that any one of the $A_{i}$ may be the starting symbol. Hence we keep track of which $A_{i}$ began the derivation in $G$ by use of the superscript $i$. We claim that $L\left(G_{j}^{\prime} \Rightarrow\right)=L\left(G^{\prime}, \Rightarrow\right)$.

Consider an arbitrary $G^{\prime}$-derivation for $x$ in $\Sigma^{\prime *}$,

$S \stackrel{b}{\Rightarrow} x$.

Either no $A_{i}, 1 \leq i \leq t$ occurs in this derivation, in which case $S \stackrel{L^{*}}{\Rightarrow} \times$ in $G_{1}^{\prime}$

since all productions in $P^{\prime}$ which are not images of A-productions are taken unchanged into $P_{i}$ or some $A_{i}$ occurs in the derivation. In this latter case consider the first appearance of an $A_{j}, 1 \leq i \leq t$, $S \stackrel{L^{*}}{\Rightarrow}{ }^{*}{ }_{i_{0}}{ }^{\alpha} i_{0} \stackrel{L}{\Rightarrow} x$ in $G^{\prime}$.

Now this $A_{i_{0}}$ yields, via images of A-productions of the type $A \rightarrow A \alpha_{j}$, a sequence of $A_{k}$ symbols, $1 \leq k \leq t$ terminated by the application of the image of an A-production of the type $A \rightarrow \beta_{\ell}$. We write this as:

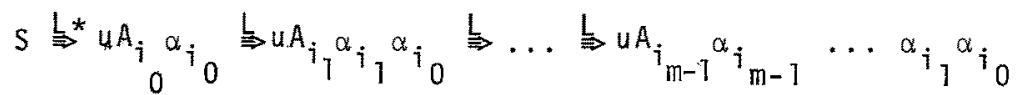

$\Rightarrow u \beta_{i_{m} \alpha_{m-1}} \cdots \alpha_{i_{1}} \alpha_{i_{0}} \Rightarrow^{*} x$

in $G^{\prime}$. Now by definition of $G_{1}^{\prime}$ the derivation 


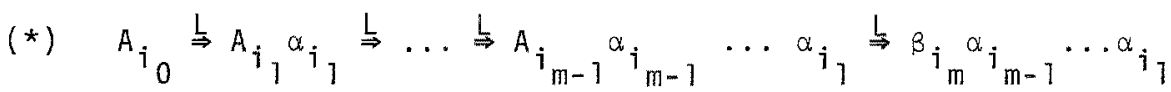

in $G$ " can be "simulated" by the derivation

(**) $\quad A_{i_{0}} \stackrel{R}{\Rightarrow} \beta_{i_{m}} A_{j_{m-1}}^{\left(i_{0}\right)} \stackrel{R}{\Rightarrow} \beta_{i_{m}} \alpha_{i_{m-1}} A_{i_{m-2}}^{\left(i_{\theta}\right)} \stackrel{R}{g} \ldots B_{i_{m}} \alpha_{j_{m}-1} \cdots \alpha_{i_{2}} A_{i_{0}}^{\left(i_{0}\right)}$

$\stackrel{\mathrm{R}}{\Rightarrow} \beta_{i_{m} \alpha_{i}-1} \cdots \alpha_{i}$.

Clearly we can prove by induction on the number of such $A_{k}$ sequences in a $G^{\prime}$-derivation $S \stackrel{L}{*}^{*} x$ in $\Sigma^{\prime} *$ that $S \Rightarrow^{*} \times$ in $G_{1}^{\prime}$. Hence

$L\left(G^{\prime}, \Rightarrow\right) \subseteq L\left(G_{\dagger}^{\prime} \Rightarrow\right)$.

Moreover, for each rightmost derivation $S \stackrel{R *}{\Rightarrow} x$ in $G_{i}^{\prime}, x$ in $\Sigma^{*}$, it can be observed that if any $A_{k}$ sequence occurs then it must, by definition of $G_{j}^{\prime}$, be of type (**). Immediately such a $(* *)$-derivation can be "simulated" in $G$ ' by the corresponding (*)-derivation. Crucial to this simulation, in both directions, is the information carried in the superscript position of the $A_{i}^{(j)}$ symbols in $(* *)$.

Hence $L\left(G_{j}^{\prime} \Rightarrow\right)=L\left(G^{\prime}, \Rightarrow\right)$ and $C l a i m ~ T$ is proved.

ctaim 2: $\quad \mathcal{Z}_{S}\left(\mathrm{G}_{1}, \Rightarrow\right) \subseteq \mathcal{Z}_{\mathrm{S}}(\mathrm{G}, \Rightarrow)$.

This proof is similar to that of claim 1, therefore we merely sketch the technique leaving the details to the reader. Consider an arbitrary interpretation $G_{j}^{\prime} \Delta G_{j}(\mu)$, where $G_{j}^{\prime}=\left(V_{j}^{\prime}, \Sigma^{\prime}, P_{j}^{\prime}, S^{\prime}\right), \mu(A)$ $=\left\{A_{1}, \ldots, A_{p}\right\}$ and $\mu(X)=\left\{X_{1}, \ldots, X_{q}\right\}$, say, for some $p, q>0$. As in Claim 1 we construct an interpretation $G^{\prime}{\underset{S}{S}}_{G}\left(\mu^{\prime}\right)$ such that $L\left(G_{1}^{\prime} \Rightarrow\right)=L\left(G^{\prime}, \Rightarrow\right)$. This is accomplished by defining $\mu^{\prime}$ to be identical to $\mu$ except that for $X, \mu^{\prime}(X)=\left\{X_{j}^{(j)}: 1 \leq i \leq q, 1 \leq j \leq p\right\}$ and introducing appropriate productions in $P$ ' such that

(+) $\quad A_{i} \stackrel{R}{\Rightarrow} \beta_{i_{0}} x_{i_{0}} \stackrel{R}{\Rightarrow} \beta_{i_{0}} \alpha_{i_{0}} x_{i_{1}} \stackrel{R}{\Rightarrow} \ldots \stackrel{R}{\Rightarrow} \beta_{i_{0}} \ldots \alpha_{i_{m}} x_{i_{m-1}} \stackrel{R}{\Rightarrow} \beta_{i_{0}} \ldots \alpha_{i_{m}}$ if $G_{i}^{\prime}$, iff

$(++) \quad A_{i} \stackrel{\llcorner}{\Rightarrow} x_{i m-1}^{(i)} \alpha_{i m} \stackrel{L}{\Rightarrow} \ldots \stackrel{L}{\Rightarrow} x_{i}^{(i)} \alpha_{i} \ldots \alpha_{i_{m}} \stackrel{L}{\Rightarrow} \beta_{i_{0}} \alpha_{i} \ldots \alpha_{i m}$ in $G^{\prime}$.

We can now state and prove the left recursion removal theorem, name 1y:

Theorem 4.9

Let $G=(V, \Sigma, P, S)$ be a c-reduced grammar form. Then there exists a c-reduced grammar form $G_{1}$ such that $G_{1}$ is non-left recursive 
and $\mathcal{L}_{\mathrm{S}}\left(G_{1}, \Rightarrow\right)=\mathcal{L}_{\mathrm{S}}(G, \Rightarrow)$

Proof: Based on Lemmas 4.7 and 4.8 the standard technique for removing left recursion from $G$ can be used. Because of Lemmas 4.7 and 4.8 the resulting grammar form $H$ is s-form equivalent to $G$ and moreover $H$ is non-left recursive, again by standard techniques. Clearly an s-form equivalent grammar form $G_{1}$ which is $c$-reduced and non-left recursive can be obtained from $H$, completing the proof.

We now prove a powerful normal form result for grammar forms which is also a normal form result for context-free grammars encompassing Chomsky and Greibach two-standard normal forms and generalizing them both considerably.

A grammar form $G=(V, \Sigma, P, S)$ is said to be a unary form if $\Sigma=\{a\}$ for some terminal a. Note that every grammar form $G$ has a g-form equivalent unary form, simply by identifying all the terminals in $G$. Similarly a grammar form $G=(V, \Sigma, P, S)$ is said to be a twosymbol form if $\Sigma=\{a\}$ for some terminal a and $V=\{S, a\}$.

Theorem 4.10 -- The Super-Normal Form Theorem

Let $G=(\{S, a\},\{a\}, P, S)$ be a two-symbol form such that

(i) $L(G, \Rightarrow)=a^{*}$, and

(ii) there exists $S \rightarrow \alpha$ in $P$ with $|\alpha|_{S} \geq 2$.

Then $\mathcal{L}_{\mathrm{S}}(G, \Rightarrow)=\mathcal{L}(C F)$.

Proof: We establish the theorem in three steps, making use of the following result from Salomaa and Soittala (1978).

Proposition: Let $E=\left(\{S, a\},\{a\}, P_{E}, S\right)$ be a two-symbol form for which $P_{E}$ includes the productions $S \rightarrow$ aSa and $S \rightarrow$ aSaSa and $L(E, \Rightarrow)=a^{*}$. Then $\mathcal{L}_{\mathrm{S}}(\mathrm{E}, \Rightarrow)=\mathcal{L}(\mathrm{CF})$.

Claim 1: Let $i \geq 0$ be an integer, $F=\left(\{S, a\},\{a\}, P_{F}, S\right)$ be a twosymbol form, $L(F, \Rightarrow)=a *$ and $P_{F}$ contain the productions $S \rightarrow a^{i} S a^{i}$ and $S \rightarrow a^{i} S^{i} S a i$. Then $\mathcal{L}_{S}(F, \Rightarrow)=\mathcal{L}(C F)$.

Proof of Claim 1: Without loss of generality we may assume $s \rightarrow a^{i}$ is in $P_{F}, 1 \leq i \leq 2 i$. We proceed in three steps. 
Step 1: If $L \subseteq \Sigma^{*}$ is an arbitrary context-free language with the property that $x$ is in $L$ implies $|x| \equiv 0$ (mod i) then we show that $L$ is in $\mathcal{L}_{s}(F, \Rightarrow)$.

Define a new alphabet $\Delta=\left\{\left[a_{j}, a_{2}, \ldots, a_{j}\right]: a_{j}\right.$ is in $\left.\Sigma, 1 \leq j \leq i\right\}$ and a homomorphism $h$ from $\Delta$ to $\Sigma^{*}$ by:

$$
h\left(\left[a_{1}, a_{2}, \ldots, a_{i}\right]\right)=a_{1} \ldots a_{i} .
$$

Let $L^{\prime}=h^{-1}(L)$. Now $L^{\prime}$ is in $\mathcal{L}(C F)$ since $\mathcal{L}(\mathrm{CF})$ is closed under inverse homomorphism. Therefore by the Proposition there is an $E^{\prime}=\left(V^{\prime}, \Delta, P_{E}^{\prime}, S^{\prime}\right){ }_{S} E$ such that $L\left(E^{\prime}, \Rightarrow\right)=L^{\prime}$.

Now extend $h$ to all symbols of $V^{\prime}$ by defining $h(A)=A$, for al1 $A$ in $V^{\prime}-\Delta$ and define $F^{\prime}=\left(V^{\prime \prime}, \Sigma, P_{F}^{\prime}, S^{\prime}\right)$ by: $V^{\prime \prime}=\left(V^{\prime}-\Delta\right) \quad 0$ s and $P^{\prime \prime}=\left\{A \rightarrow h(\alpha): A \rightarrow \alpha\right.$ in $\left.P_{E}^{\prime}\right\}$. Since $E^{\prime} \Delta \vec{s} E$ it should be clear that $F^{\prime} \rightarrow F$. Moreover $L\left(F^{\prime}, \Rightarrow\right)=h\left(L\left(E^{\prime}, \Rightarrow\right)\right)=h\left(L^{\prime}\right)=L$. Hence $L$ is in $\mathcal{L}_{\mathrm{s}}(\mathrm{F}, \Rightarrow)$ as desired.

Step 2: If $L$ is an arbitrary language in $\mathcal{L}_{\mathrm{s}}(F, \Rightarrow)$ and $x, y, z, w$ are arbitrary words over some alphabet $\bar{\Sigma}$ with $|x|=|z|=|w|=i$ and $|y|=j$, for some $j, 0 \leq j \leq i-1$, then we show that $L^{\prime}=x y z$ is also in $\mathcal{Z}_{s}(F, \Rightarrow)$.

By assumption there is an $F^{\prime}=\left(V^{\prime}, \Sigma^{\prime}, P_{F}^{\prime}, S^{\prime}\right) \Delta{ }_{S} F$ with $L\left(F^{\prime}, \Rightarrow\right)=L$. Let $A$ and $\vec{S}$ be two new nonterminals and consider $F^{\prime \prime}=\left(V^{\prime} \cup\{\bar{S}, A\} \cup \bar{\Sigma}, \Sigma^{\prime} \cup \bar{\Sigma}, P_{F^{\prime \prime}}, \bar{S}\right)$, where $P_{F}^{\prime \prime}=P_{F}^{\prime} \cup\left\{\bar{S} \rightarrow x A z S^{\prime} w ; A \rightarrow y\right\}$. Clearly $F^{\prime \prime} \rightarrow F$ and $L\left(F^{\prime \prime}, \Rightarrow\right)=x y z L=L^{\prime}$.

Step 3: We now show that if $L$ is an arbitrary context-free language then $L$ is in $\mathcal{Z}_{\mathrm{s}}(\mathrm{F}, \Rightarrow)$.

We can write $L=\left[\cup \bigcup_{j=0}^{i-1} L_{j}\right.$, where each $L_{j}$ is the finite union of languages $L^{\prime}=x y z L w$, where $|x|=|z|=|w|=i,|y|=j$ and $u$ is in $L$ implies $|u| \equiv 0(\bmod i)$. Since all such languages $L$ 'are in $\mathcal{L}_{S}(F, \Rightarrow)$ by step $2, \mathcal{L}_{S}(F, \Rightarrow)$ contains all finite languages and $\mathcal{L}_{S}(F, \Rightarrow)$ is closed under union the result follows.

This completes the proof of Claim 1 .

Claim 2: Let $i, j \geq 0$ be integers, $H=\left(\{S, a\},\{a\}, P_{H}, S\right), L(H, \Rightarrow)=a *$ and $\mathrm{P}_{H}$ contain $S \rightarrow a^{i} S_{a}{ }^{j} S^{k}$. Then $\mathcal{L}_{S}(H, \Rightarrow)=\mathcal{L}(C F)$.

Proof of ctaim 2: We reduce this claim to the previous one. H contains the productions $S \rightarrow a$ and $S \rightarrow a^{i} S a^{j} S a^{k}$, for some $i, j, k \geq 0$. Hence we have: 
(*) $S \Rightarrow{ }^{*} a^{i} 1_{S a}{ }^{i_{2}} S_{S a}{ }^{i} S_{S a}{ }^{i}{ }^{S} a^{i} 5_{S a}{ }^{i} 6$ in $H$, for some $i_{1}, \ldots, i_{6}$.

Let $t=\max \left(\left\{i_{1}, \ldots, i_{6}\right\}\right)$ and $m=4 t+3$. Since $s \Rightarrow^{*} a^{k}$, for all $k \geq 1$ there exist derivations

$$
\begin{aligned}
& a^{i} \mathrm{Sa}^{i} 2 \Rightarrow \mathrm{a}^{\mathrm{m}} \text {, and } \\
& a^{i^{3}} \mathrm{Sa}^{i^{i}}{ }_{\mathrm{Sa}}{ }^{i} \mathrm{Sa}^{{ }^{i} 6} \Rightarrow a^{\mathrm{m}} \text { in } \mathrm{H} \text {. }
\end{aligned}
$$

This follows because $i_{i}+i_{2} \leq 4 t$ and $i_{3}+i_{4}+i_{5}+i_{6} \leq 4 t$.

Hence $S \Rightarrow{ }^{*}{ }^{m} a^{m}$ in $H$ is obtained from (*).

Second, there exist derivations

$$
\begin{aligned}
& a^{i_{1}} \mathrm{Sa}^{i^{2}} \Rightarrow a^{m} \text {, } \\
& a^{i^{3}} \mathrm{Sa}^{i^{i}}{ }^{a^{i}}{ }^{5} \mathrm{Sa}^{i_{6}} \Rightarrow a^{m} \text { and } \\
& a^{m} \text { in } H \text {. }
\end{aligned}
$$

Hence, from (*) we obtain:

$$
\mathrm{S} \Rightarrow{ }^{*} \mathrm{~m}^{\mathrm{m}} \mathrm{m}^{\mathrm{m}} \mathrm{Sa}{ }^{\mathrm{m}} \text { in } \mathrm{H} \text {. }
$$

We have shown that $H$ simulates $E$. Thus $\mathcal{L}_{S}(E, \Rightarrow) \subseteq \mathcal{L}_{s}(H, \Rightarrow)$ and therefore $\mathscr{L}_{S}(H, \Rightarrow)=\mathscr{L}^{\prime}(\mathrm{CF})$. Claim 2 is thereby established.

Returning to the proof of the theorem, we consider the grammar form $G$.

$$
\text { Now } S \rightarrow \alpha \text { is in } P \text { with }|\alpha|_{S} \geq 2 \text {. Hence } \alpha=a^{i}{ } S_{a^{i}}{ }^{i} \ldots a^{i} t_{S a}{ }^{i} t+1 \text {, }
$$

for some $t \geq 2$ and $i_{p} \geq 0, i \leq p \leq t+1$. Immediately, we obtain the derivation:

$$
S \Rightarrow a^{i} S a^{j} S a^{k} \text { in } G
$$

for some $i, j, k \geq 0$. Therefore $G$ simulates the grammar form $H$ of claim 2 giving the desired result.

We now extend Theorem 4.10 to g-grammatical families.

$$
\text { We say } F=(\{S, a\},\{a\}, P, S) \text {, a two-symbol form, is a normal }
$$

form grammar if $L(F, \Rightarrow)=a$ * and there is a production $S \rightarrow \alpha$ in $P$ with $\alpha=a^{i} S \ldots a^{i} \mathrm{~m}$, with $m \geq 2$.

our major theorem for g-interpretations now follows.

\section{Theorem 4.11}

Let $F$ be a normal form grammar and $G$ a grammar form. Then there exists $H, g$-form equivalent to $G$ such that $H \Delta F$. 
Proof: Without loss of generality, we may assume $G=\left(V_{G},\{a\}, P G, S_{G}\right)$. There are two cases to consider.

Case 1: $G$ is finite. Then $\mathcal{L}_{\mathrm{g}}(G, \Rightarrow)=\{\emptyset\}$ or $\mathcal{L}(\mathrm{FIN})$. In both cases there exists $H \underset{S}{\Delta} F$ g-form equivalent to $G$ since $F$ can be assumed to contain the production $\mathrm{S} \rightarrow \mathrm{a}$.

Case 2: G is infinite. There are two subcases.

Case 2.1: $G$ is a two-symbol form. Then $\mathcal{L}_{\mathrm{g}}(G, \Rightarrow)=\mathcal{L}$ (REG), $\mathcal{L}$ (LIN) or $\mathcal{L}(C F)$, by Theorem 2.18. If $\mathcal{L}_{g}(G, \Rightarrow)=\mathcal{L}(C F)$ take $H$ to be equal to F. If $\mathcal{L}_{\mathrm{g}}(G, \Rightarrow)=\mathcal{L}(R E G)$ let the productions $S \rightarrow a ; A \rightarrow a ; S \rightarrow a^{i_{A a}}{ }^{i_{2}} \ldots a^{i_{m-1}}{ }_{A a}{ }^{i_{m}}$ S define $H$. Clearly $H \underset{s}{\triangleleft} F$ and $\mathcal{L}_{g}(H, \Rightarrow)=\mathcal{L}$ (REG) since $H$ is non-self-embedding.

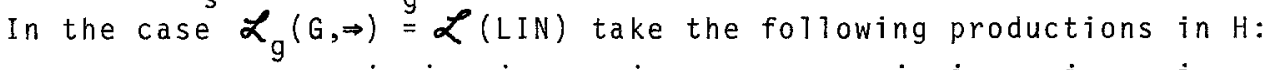
$S \rightarrow a ; A \rightarrow a ; S \rightarrow a^{i} 1_{B}{ }^{i}{ }_{A a}{ }^{i}{ } A \ldots a{ }^{i} m_{A} ;$ and $B \rightarrow a^{i} 1_{A}{ }^{i} 2 \ldots a^{i} m^{-1} A a^{i} m_{S}$. The two step delay in reproducing $S$ ensures $S$ is self-embedding, even when $i_{1}=\ldots=i_{m}=0$.

Case 2.2: G is not a two-symbol form. Since $G$ is unary this implies $G$ has at least two nonterminals. By Theorems 4.6 and 4.9 we can assume $G$ is both s-reduced and non-left recursive. Observe that if $G$ is expansive then $\mathcal{L}_{\mathrm{g}}(G, \Rightarrow)=\mathcal{L}(\mathrm{CF})$ and we can take $H$ equal to $\mathrm{F}$. Hence we only need deal with the case of $G$ being non-expansive. Letting $\#\left(V_{G}-\{a\}\right)=n$ we proceed by induction on $n$. The basis $n=1$ has been dealt with under Case 2.1. Assume the theorem is true for all $\bar{G}$ with $1 \leq \#\left(V_{\bar{G}}-\{a\}\right) \leq \ell$, for some $\ell \geq 1$. Consider the case of $G$ with $\#\left(V_{G}-\{a\}\right)=n=\ell+1$.

By the discussion above each reduced subgrammar $G_{A}$ for $A \neq S_{G}$ and A appearing on the right side of an $S_{G}$-production has fewer than $n$ nonterminals. Therefore by the inductive assumption there is an $F_{A} \underset{s}{\Delta}$ with $\mathcal{L}_{g}\left(F_{A} \Rightarrow\right)=\mathcal{Z}_{g}\left(G_{A}, \Rightarrow\right)$ and by Lemma 2.19 we can assume $G_{A}$ and $F_{A}$ are identical.

We construct $H$ by first taking all productions of $P_{G}$ into $P_{H}$ with the exception of the $S_{G}$-productions. Second for each $S_{G}$-production $S_{G} \rightarrow \alpha$ we add appropriate productions to $P_{H}$ as follows:

(i) If $\alpha=a^{j}$ for some $j \geq 1$ take $S_{G} \rightarrow a$ into $P_{H}$.

(ii) If $a=a^{j}{ }_{1} A_{1} a^{j} \ldots a^{j} k_{A_{k}} a^{j} k+1$ for some $k \geq 1$ add sufficient productions to $P_{H}$ to "simulate" this production. Letting 
$p$ be the least integer such that $p(m-1) \geq 2 k$ consider the p-step derivation

$$
\begin{aligned}
& s \stackrel{R}{\Rightarrow} a^{i}{ } S_{a}{ }^{i}{ }^{2} \ldots a^{i} m_{S} \stackrel{R}{\Rightarrow} a^{i} 1 S \ldots a^{i} m^{+i} 1_{S} \ldots a^{i} m_{S} \stackrel{R}{\Rightarrow} \ldots \\
& \ldots \stackrel{\mathrm{R}}{\Rightarrow} \mathrm{a}^{\mathrm{i}} \mathrm{r}_{\mathrm{S}} \ldots \ldots \mathrm{a}^{\mathrm{i}} \mathrm{m}_{\mathrm{S}}
\end{aligned}
$$

in $F$ using the production $S \Rightarrow a^{i} S \ldots a^{i} m_{S}$. An isolated version of this derivation is used to "simulate" $S_{G} \rightarrow \alpha$. First add the following productions to $\mathrm{P}_{H}$ :

$$
\begin{aligned}
& S_{G} \rightarrow a^{i} D_{D_{1}} a^{i^{2} \ldots a^{i} m-1} D_{m-1} a^{i} m_{B_{1}} \text {, } \\
& B_{1} \rightarrow a^{i} 1_{D_{m}} a^{i} 2 \ldots a^{i} m-1 D_{2 m-2} a^{i} m_{B_{2}} \text {, } \\
& B_{p-2} \rightarrow a^{i} D_{(p-2)(m-1)+1} a^{i_{2} \ldots a^{i} m-1} D_{(p-1)(m-1)} a^{i} B_{p-1} .
\end{aligned}
$$

Second, the production

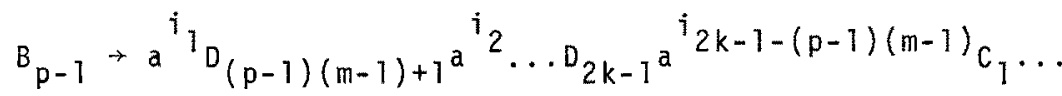

$$
\begin{aligned}
& \cdots \rightarrow a^{i} m-1 C_{p(m-1)-2 k+1} a^{i} m_{D_{2 k}} \text {, } \\
& \text { if } j_{k+1}=0 \text { and the production } \\
& B_{p-1} \rightarrow a^{i} D_{(p-1)(m-1)+1} a^{i} D(p-1)(m-1)+2 \cdots \\
& \ldots \quad D_{2 k+1}{ }^{i} 2 k+1-(p-1)(m-1) c_{1} \ldots \\
& \ldots \quad a^{i} m-1 c_{p(m-1)-2 k-1}{ }^{i}{ }^{i} C_{p(m-1)-2 k} \text {, } \\
& \ldots \quad a^{i_{m-1}} C_{p(m-T)-2 k-1} a^{i} c_{p(m-1)-2 k}, \\
& \mathrm{C}_{\mathrm{q}} \rightarrow \mathrm{a} \text {, for all new nonterminals } \mathrm{C}_{\mathrm{q}} \\
& D_{2 q-1} \rightarrow a \text {, for all new nonterminals } D_{2 q-1}, q \geq 1 \text {. }
\end{aligned}
$$

The $B_{q}$ are also new nonterminals, however the even subscripted $D_{2 q}$ are not new, in fact $D_{2 q}=A_{q}, 1 \leq q \leq k$.

The basic idea behind this construction is that the $D_{2 q-1}$ represent the $a^{j} q$, the $D_{2 q}$ are the $A_{q}$ and if $j_{k+1}=0$ then we must 
ensure that $D_{2 k}=A_{k}$ also appears rightmost in the simulation, in case $A_{K}=S_{G}$. On the other hand if $j_{k+1} \neq 0$ then $A_{k}$ must not appear rightmost in the simulation, hence in this case we also add $D_{2 k+1}$ to represent $a^{j} k+1$. We can do this since there are at least $p+1 \leq 2 k+1$ nonterminals in the underlying $p$-step derivation in $F$.

clearly $H \underset{S}{\Delta} F$ by construction. Therefore it only remains to prove that $H$ and $G$ are $g$-form equivalent.

First construct $H^{\prime} \vec{g} H$ in which all the productions in $P_{H}$ which occur in $P_{G}$ are taken unchanged into $P_{H^{\prime}}$. This leaves the productions in $P_{H}$ which are used to "simulate" the $S_{G}$-productions of $G$. For each production $S_{G} \rightarrow \alpha$ in $G$ :

(i) If $\alpha=a^{j}$, for some $j \geq 1$, then take $S_{G} \rightarrow \alpha$ into $P_{H^{\prime}}$.

This is possible since $S_{G} \rightarrow a$ is in $P_{H}$.

(ii) If $\alpha=a^{j}{ }^{j} A_{1} \ldots A_{k} a^{j} k+1$ for some $k \geq 1$ take into $P_{H}$, the simulating productions introduced above except that a is replaced by $\lambda, c_{q} \rightarrow \lambda$ is taken for all $q$ and $D_{2 q-1} \rightarrow a^{j} q$ is taken for all $q$.

It should be clear that $H^{\prime}$ simulates $G$, hence

$\mathcal{L}_{g}(G, \Rightarrow) \subseteq \mathcal{L}_{g}\left(H^{\prime}, \Rightarrow\right) \subseteq \mathcal{Z}_{g}(H, \Rightarrow)$. We now prove that $\mathcal{L}_{g}(H, \Rightarrow) \subseteq \mathcal{L}_{g}(G, \Rightarrow)$. We do this in two steps.

Step 1: Construct $\bar{H}$ from $H$ be removing all the nonterminals not in $V_{G}-\{a\}$ using the back-substitution lemma. Hence $\bar{H}=\left(V_{G},\{a\}, \bar{P}, S_{G}\right)$ and by Lemma 2.12 is $g$-form equivalent to $H$. Moreover all the non- $S_{G}{ }^{-}$ productions in $\bar{P}$ are identical to those in $P_{G}$ and the $S_{G}$-productions in $\bar{P}$ are "similar" to those in $P_{G}$.

Step 2: Consider the "similarity" of the $S_{G}$-productions in $\bar{P}$ and $P_{G}$. Let $S_{G} \rightarrow \alpha$ be a production in $\bar{P}$. Then either

(i) $\alpha=a$ and there is some production $S_{G} \rightarrow a, i \geq 1$ in $P_{G}$, or

(ii) $a=a^{\ell} 1_{A_{1}} \ldots A_{k} a^{\ell} k+1$ for some $k \geq 1$ and by the construction each of the $l_{q}, 1 \leq q \leq k+1$ are non-zero. By the construction there is some production $S_{G} \rightarrow \beta$ in $P_{G}$ with $\beta=a^{j}{ }_{A_{1}} \ldots A_{k} a^{j} k+1$ and $j_{q} \geq 0,1 \leq q \leq k+1$.

If $j_{q}>0,1 \leq q \leq k+1$, then we have found the corresponding production in $P_{G}$. Otherwise we modify $S_{G} \rightarrow B$ in $\bar{P}$ so that there is a corresponding production $S_{G} \rightarrow \alpha$ in $P$. We need the following: 
Claim: Let $S_{G} \rightarrow \beta=a^{\ell} 1_{A} \ldots A_{k}{ }^{{ }^{l}}{ }^{k} k+1, k \geq 1,1 \leq q \leq k+1$, be $a$ production in $\bar{H}$, and $r$ an integer $1 \leq r \leq k+1$. Replace $S_{G} \rightarrow B$ by $S_{G_{-}} \rightarrow a^{l}{ }^{l} A_{1} \ldots A_{r-1} A_{r^{a}}{ }^{l} k+1$ where $a^{l} r$ has been erased in $B$. Let this be $\bar{H}^{\prime}$. Then $\bar{H}$ and $\bar{H}^{\prime}$ are g-form equivalent if ejther $A_{r-1}$ or $A_{r}$ is not equal to $S_{G}$.

Proof of Claim: Assume $A_{r}$ is not equal to $S_{G}$. Then, by the assumptions of the theorem the subgrammar form $\bar{H}_{A_{r}}$ defines a g-grammatical family, which is a full semi-AFL (see Section 4.2) and such families are closed under pre- or post-product with a finite set. Hence taking a ${ }^{2} r$ into the subgrammar defined by $A_{r}$ does not lose any generative capacity, hence $\bar{H}$ and $\bar{H}^{\prime}$ are g-form equivalent. The full details of this proof are left to the reader.

Returning to step 2 consider a production $S_{G} \rightarrow \alpha$ which contains $A_{1}, \ldots, A_{k}$ in this order, that is $\alpha={ }^{j}{ }^{j} A_{1} \ldots A_{k}{ }^{i} k+1$. Since $S_{G} \rightarrow \alpha$ does not correspond to $S_{G} \rightarrow B$ this immediately implies there are some $j_{r}=0,1 \leq r \leq k+1$. Replace $S_{G} \rightarrow \beta_{Q_{r}}^{\text {in }} \bar{P}$ by the production $S_{G} \rightarrow \bar{B}$ where $\bar{\beta}$ is the same as $\beta$ except that $a^{2}$ is replaced by $\lambda$ if $a^{j} r=\lambda$. Now since $B$ can contain at most one appearance of $S_{G}$ then such an $a^{\ell} r$ will be adjacent to a non- $S_{G}$ nonterminal with the exception of the following two cases: (a) $r=1$ and $A_{1}=S_{G}$ and (b) $r=2$, $A_{1}=S_{G}$ and $k=1$. However case (a) would imply $i_{1}=0$ and $A_{1}=S_{G}$ in $S_{G} \rightarrow \alpha$ in $G$. But by assumption $S_{G}$ is non-left recursive hence this case will not occur. Similarly in case (b) since $a^{\ell_{2}} \neq \lambda$ the construction must have given either $a^{j} \neq \lambda$ or there exists another $S_{G}$-production in $G$ of the form $S_{G} \rightarrow a{ }^{q} T_{G}{ }_{G}{ }^{q_{2}}$ with $q_{1}, q_{2}>0$.

Hence in all cases we can modify the $S_{G}$-productions in $\bar{H}$ without losing any generative capacity to obtain a production which has a corresponding production in $G$.

It should be clear that $G \underset{g}{A} \bar{H}$ and we have also shown that $\bar{H} \rightarrow G$, hence $G$ and $\bar{H}$ are strong $g$-form equivalent. Therefore $H$ and $G$ are $g$-form equivalent, completing the proof. 
Immediate consequences of Theorem 4.11 are the following normal form results.

Let $F_{1}$ be defined by: $S \rightarrow$ a; $S \rightarrow$ a $S ; S \rightarrow$ aSS,

$F_{2}$ be defined by: $S \rightarrow$ a; $S \rightarrow S S$

and $\quad \mathrm{F}_{3}$ be defined by: $\mathrm{S} \rightarrow \mathrm{a} ; \mathrm{S} \rightarrow \mathrm{aS} ; \mathrm{S} \rightarrow$ aSaSaS

then each $F_{i}$ is a normal form grammar, $1 \leq i \leq 3$. $F_{1}$ is the Greibach two-standard form grammar, and $F_{2}$ the Chomsky normal form grammar. Hence

\section{Theorem 4.12}

Let $G$ be a non-empty grammar form, then there exist grammar forms $H_{1}$ and $H_{2}$ g-form equivalent to $G$ such that

(i) $\mathrm{H}_{1}$ is in Greibach two-standard normal form.

(ii) $\mathrm{H}_{2}$ is in Chomsky normal form.

However consider $\mathrm{F}_{4}$ defined by $\mathrm{S} \rightarrow \mathrm{a}^{i}, 1 \leq i \leq 4 ; \mathrm{S} \rightarrow$ aSaSa, the Griebach-Nivat "normal form" grammar. Note that $F_{4}$ does not fulfi the conditions of Theorem 4.11. Although $\mathcal{L}_{\mathrm{g}}\left(\mathrm{F}_{4} \Rightarrow\right)=\mathcal{Z}(\mathrm{CF})$ not all g-grammatical families can be characterized by s-interpretations of $\mathrm{F}_{4}$. Since every infinite interpretation $\mathrm{F}_{4} \underset{\mathrm{s}}{\mathrm{F}_{4}}$ is self-embedding, then $\mathcal{L}_{\mathrm{g}}\left(\mathrm{F}_{4}, \Rightarrow\right) \neq \mathcal{L}(\mathrm{REG})$. Hence $\mathcal{L}(\mathrm{REG})$ cannot be obtained as the g-grammatical family of a grammar form $H$ with $H_{\text {in }} F_{4}$-normal form. Whether this is the only family that cannot be obtained from $\mathrm{F}_{4}$ is an open question. Moreover whether there exist normal form grammars $F$ which miss an arbitrary g-grammatical family also remains an open question.

\section{II.4.2 Closure Properties}

In this section we demonstrate that $\mathcal{L}_{\mathrm{S}}(G, \Rightarrow)$ is closed under intersection with regular sets and, in general, is not closed under any of the other AFL operations. In contrast we prove that $\mathcal{Z}_{g}(G, \Rightarrow)$ is a full semi-AFL for all infinite grammar forms $G$.

Consider the grammar form $G$ defined by the production $S \rightarrow a b$, then $\mathcal{L}_{\mathrm{S}}(\mathrm{G}, \Rightarrow) \neq \mathcal{L}(\mathrm{FIN})$ by Theorem 2.5 and further for each $L$ in $\mathscr{L}_{S}(G, \Rightarrow), L$ contains only words of length two in each word of which the first symbol is not equal to the second symbol. Moreover for each $a_{1} a_{2}, b_{1} b_{2}$ in $L, a_{1} \neq b_{2}$ and $a_{2} \neq b_{1}$ because s-interpretations are given by dfl-substitutions. Hence $\mathcal{L}_{s}(G, \Rightarrow)$ is not closed under homomorphism, since $\{a a\}$ is not in $\mathcal{L}_{5}(G, \Rightarrow)$, and is not closed under union since $\{a b, b a\}$ is not in $\mathcal{Z}_{S}(G, \Rightarrow)$. Since $G$ only gives rise to finite sets $\mathcal{K}_{s}(G, \Rightarrow)$ is not closed under inverse homomorphism. 
Finally, since all words in each $L$ in $\mathcal{L}_{s}(G, \Rightarrow)$ are of length two, $\mathcal{L}_{S}(G, \Rightarrow)$ is not closed under catenation. clearly $\mathcal{L}_{S}(G, \Rightarrow)$ is closed under intersection with regular sets.

These preliminary remarks give rise to the following:

\section{Theorem 4.13}

Let $G=(V, \Sigma, P, S)$ be a grammar form. Then $\mathcal{K}_{S}(G, \Rightarrow)$ is closed under intersection with regular sets and under dfl-substitution, but in general, under no other AFL operations.

Proof: Since the standard construction assumes closure under union it has to be modified slightly. Assume $G$ is in Chomsky normal form without any loss of generality (Theorem 4.12). Let $G^{\prime}=\left(V^{\prime}, \Sigma^{\prime}, P^{\prime}, S^{\prime}\right){\underset{S}{S}}_{G}$ and $M=\left(Q, \Sigma^{\prime}, \delta, q_{0}, F\right)$ be an arbitrary finite state acceptor. We construct an interpretation $G^{\prime \prime} \Delta G(\mu)$ such that $L\left(G^{\prime \prime}, \Rightarrow\right)=L\left(G^{\prime}, \Rightarrow\right) \cap L(M)$.

$$
\text { Let } V^{\prime \prime}=\left\{S^{\prime}\right\} \Sigma^{\prime} \cup\left\{[p, A, q]: A \text { in } V^{\prime}-\Sigma^{\prime}, p, q \text { in } Q\right\}
$$

and $G^{\prime \prime}=\left(V^{\prime \prime}, \Sigma^{*}, P^{\prime \prime}, S^{\prime}\right)$. Define $P^{\prime \prime}$ as those productions obtained by taking:

(a) for each production $S^{\prime} \rightarrow A B$ in $P^{\prime}$, all productions

$S^{\prime} \rightarrow\left[q_{0}, A, p\right][p, B, q]$ with $p$ in $Q$ and $q$ in $F$,

(b) for each production $S^{\prime} \rightarrow$ a in $P^{\prime}$, the production $S^{\prime} \rightarrow$ a only if a is in $L(M)$,

(c) for each production $A \rightarrow B C$ in $P^{\prime}$, the productions $[p, A, q] \rightarrow[p, B, r][r, C, q]$ for a $11 p, q, r$ in $Q$, and

(d) for each production $A \rightarrow$ a in $P^{\prime}$, the productions $[p, A, q] \rightarrow$ a for a $11 p, q$ in $Q$ with $\delta(p, a)=q$.

Clearly $L\left(G^{\prime \prime} \Rightarrow\right)=L\left(G^{\prime}, \Rightarrow\right) \cap L(M)$ by standard methods, and moreover letting $\mu(a)=\{a\}$, for all a in $\Sigma^{\prime}, \mu(A)=\{A\} \cup\{[p, A, q]: p, q$ in $Q\}$ then $G^{\prime \prime} \Delta_{S} G^{\prime}(\mu)$ and therefore $L\left(G^{\prime \prime}, \Rightarrow\right)$ is in $\mathscr{L}_{S}(G, \Rightarrow)$ às required.

closure under dfl-substitution follows directly from the definition of s-interpretation. The third part of the Theorem follows from the preliminary remarks, completing the proof.

In contrast we now prove that every g-grammatical family is not only closed under intersection with regular sets but also under union and homomorphism. 


\section{Theorem 4.14}

Let $G=(V, \Sigma, P, S)$ be a grammar form. Then $\mathcal{L}_{g}(G, \Rightarrow)$ is closed under intersection with regular sets, union and homomorphism and, in genera1, under no other AFL operations.

Proof: By Corollary $1.2 \mathcal{L}_{g}(G, \Rightarrow)=\mathbb{Z}\left(\mathcal{L}_{s}(G, \Rightarrow)\right)$, therefore $\mathcal{W}\left(\mathcal{L}_{g}(G, \Rightarrow)\right)=\mathcal{W}\left(\mathcal{W}\left(\mathcal{L}_{s}(G, \Rightarrow)\right)\right)=\mathcal{L}_{g}(G, \Rightarrow)$. Let $G_{j}=\left(V_{i}, \Sigma_{i}, P_{j}, S_{j}\right)$, $i=1,2$ be two $g$-interpretations of $G, G_{i} \Delta G\left(\mu_{i}\right), i=1,2$. Without loss of generality we may assume $S_{1}=S_{2}$ and $\left(V_{1}-\Sigma_{1}\right) \cap\left(V_{2}-\Sigma_{2}\right)=\left\{S_{1}\right\}$, and further assume that $S_{1}$ does not appear on the right hand side of any production in $P_{1} \cup P_{2}$. (this transformation can easily be accomplished within $\left.g_{g}(G)\right)$. Now letting $G^{\prime}=\left(V_{1} \cup V_{2}, \Sigma_{1} \cup \Sigma_{2}, P_{1} \cup P_{2}, S_{1}\right)$ we have $G^{\prime} \underset{g}{\Delta} G$ and $L\left(G^{\prime}, \Rightarrow\right)=$ $L\left(G_{1}, \Rightarrow\right) \cup L\left(G_{2}, \Rightarrow\right)$. We can prove closure under intersection with regular sets using a slightly modified version of the construction given in Theorem 4.13 .

Consider $G$ defined by $S \rightarrow$ a, then $\mathscr{L}_{g}(G, \Rightarrow)=\mathscr{L}(F I N)$ and is therefore not closed under catenation closure nor under inverse homomorphism. Finally, $G$ defined by $S \rightarrow$ a and $S \rightarrow$ asa has $\mathcal{L}_{\mathrm{g}}(\mathrm{G}, \Rightarrow)=\mathcal{L}$ (LIN) by Theorem 2.18 and $\mathcal{L}$ (LIN) is not closed under catenation.

However if we now restrict our attention to infinite grammar forms, we obtain full semi-AFLs under g-interpretation, although under s-interpretation we cannot strengthen Theorem 4.13 in this case. For example, let $G$ be defined by the productions

$$
S \rightarrow a b ; S \rightarrow a b S a b
$$

then by similar arguments to those used previously $\mathcal{L}_{\mathrm{s}}(G, \Rightarrow)$ is not closed under union nor under homomorphism, and since $\mathcal{L}_{s}(G, \Rightarrow) \subseteq \mathcal{L}$ (LIN) and contains non-regular languages, $\mathcal{L}_{\mathrm{s}}(G, \Rightarrow)$ is not closed under either catenation or catenation closure. Let $h$ be defined by $h(a)=a b$ and $h(b)=\lambda$ then $h^{-1}(a b)=b^{*} a b^{*}$ is not in $\mathcal{L}_{s}(G, \Rightarrow)$, hence $\mathcal{L}_{s}(G, \Rightarrow)$ is not closed under inverse homomorphism.

\section{Theorem 4.15}

Let $G=(V, \Sigma, P, S)$ be an infinite grammar form, then $\mathcal{L}_{\mathrm{g}}(G, \Rightarrow)$ is a full semi-AFL. 
Proof: Because of Theorem 4.14 it suffices to show that $\mathcal{L}_{\mathrm{g}}(G, \Rightarrow)$ is closed under regular substitution. By Theorem 4.6 we may assume that $G$ is $s$-reduced and that $G$ is a unary form without loss of generality. We proceed by induction on the number of nonterminals in $V-\Sigma$. Suppose $G$ has $k$ nonterminals, $k \geq 1$. If $k=1$ then $G$ is a two-symbol form, hence $\mathcal{Z}_{\mathrm{g}}(G, \Rightarrow)=\mathcal{X}(\mathrm{REG}), \mathcal{Z}(\mathrm{LIN})$ or $\mathcal{L}(\mathrm{CF})$ by Theorem 2.18. In each case $\mathcal{L}_{\mathrm{g}}(G, \Rightarrow)$ is closed under regular substitution. Assume the resuit holds for all $k \leq t, t \geq 1$, we now prove that it holds for the case $k=t+1$.

Let $V-\Sigma=\left\{A_{1}, \ldots, A_{k}\right\}$ where $A_{1}=S$ and $A_{j} \Rightarrow^{+} \alpha A_{j} B$ in $G$ implies $i \leq j$. Let $G^{\prime}=\left(V^{\prime}, \Sigma^{\prime}, P^{\prime}, S^{\prime}\right) \Delta \vec{g} G(\mu)$ and assume $G^{\prime}$ is reduced. Let $\tau$ be a regular substitution on $\Sigma^{\prime *}$ and $\Sigma_{\tau}$ be the image alphabet. Our ajm is to construct a $G_{T}=\left(V_{\tau}, \Sigma_{\tau}, P_{\tau}, S^{\prime}\right)$ such that $L\left(G_{\tau}, \Rightarrow\right)=\tau\left(L\left(G^{\prime}, \Rightarrow\right)\right)$ and $G_{\tau} \vec{g} G$. Observe that for all $i, 2 \leq i \leq k, G_{i}=\left(V, \Sigma, P, A_{i}\right)$ has at most $k$ - 1 nonterminals when reduced and hence $\mathcal{L}_{g}\left(G_{j} \Rightarrow\right)$ is closed under regular substitution, by the inductive assumption. Also note that for al1 $S \rightarrow \alpha$ in $P$ we can assume $\alpha$ contains at most one $S$, otherwise $\mathscr{L}_{g}(G, \Rightarrow)=\mathcal{Z}(C F)$ by Theorem 2.15 and the result follows trivialiy.

Since for each $i, 2 \leq i \leq k, \mathcal{Z}_{g}\left(G_{i}, \Rightarrow\right)$ is closed under regular substitution, it is a full semi-AFL and therefore it is closed under pre- and post-product with a regular set, that is for a $11 \mathrm{~L}$ in $\mathcal{L}_{g}\left(G_{i}, \Rightarrow\right)$ and $R$ a regular set, $L R$ and $R L$ are in $\mathcal{L}_{g}\left(G_{i}, \Rightarrow\right)$.

If there is a derivation $S \Rightarrow^{*} x$ Sy, $x y \neq \lambda^{g}$ in $G$ then we may assume there are productions $S \rightarrow x S y$ and $S \rightarrow \lambda$ in $G$, and therefore letting $G_{S}=(\{S, a\},\{a\},\{S \rightarrow \lambda, S \rightarrow x S y\}, S), \mathcal{L}_{g}\left(G_{S}, \Rightarrow\right) \geq \mathcal{L}(\operatorname{REG})$ by Lemma 2.13 and if $x \neq \lambda$ and $y \neq \lambda, \mathcal{L}_{g}\left(G_{S}, \Rightarrow\right) \equiv \mathcal{L}$ (LIN) by Theorem 2.16 .

We use each of these remarks in the construction of $G_{\tau}$. Given $G^{\prime}$ and $\tau$, consider the effect of replacing $P^{\prime}$ by $\tau\left(P^{\prime}\right)$, where $\tau(A)=\{A\}$, for all $A$ in $V^{\prime}-\Sigma^{\prime}$, and $\Sigma^{\prime}$ by $\Sigma_{\tau}$, giving $\tau\left(G^{\prime}\right)$ say. Clearly $L\left(\tau\left(G^{\prime}, \Rightarrow\right)\right)=\tau\left(L\left(G^{\prime}, \Rightarrow\right)\right.$. However $G^{\prime}$ has an infinite set of productions. We now modify $\tau\left(G^{\prime}\right)$ to give a $G_{\tau}$ with a finite set of productions.

Let $A \rightarrow \alpha$ belong to $P^{\prime}$, where $A$ is in $\mu(S)$. Now $A \rightarrow$ a may be written as:

either (i) $A \rightarrow x, x$ is in $\Sigma^{\prime} *$

or (ii) $A \rightarrow \alpha_{1} \ldots \alpha_{m}, m \geq 1$ and $\alpha_{i}=x_{i} B_{i} y_{i}$.

Construct $\mu_{\tau}$ and $P_{\tau}$ such that:

Case (i) gives rise to derivations $A \stackrel{+}{\Rightarrow}$ in $G_{\tau}, y$ in $\tau(x)$. This can be done since $G$ is infinite. 
Case (ii) gives rise to derivations

$A \Rightarrow z_{1} \ldots z_{m}$ in $G_{\tau}$, where $z_{i}$ is in $\tau\left(x_{i}\right) \tau\left(L\left(B_{i}, \Rightarrow\right)\right) \tau\left(y_{i}\right)$, $1 \leq i \leq m$. If $B_{j}$ is not in $\mu(s)$ then by

the inductive assumption and the remarks above, replace $\alpha_{i}$ by $\alpha_{i}^{\prime}$ the sentence symbol of a sub-grammar generating $\tau\left(x_{i}\right) \tau\left(L\left(B_{i}, \Rightarrow\right)\right) \tau\left(y_{i}\right)$. This sub-grammar can

be obtained as a $g$-interpretation of $G_{j}$, where $B_{j}$ is in $\mu\left(A_{j}\right), j \geq 2$, since $\mathcal{L}_{g}\left(G_{j} \Rightarrow\right)$ is a full semi-AFL

by the inductive assumption.

If $B_{i}$ is in $\mu(S)$, then four cases occur:

(a) $x_{i}=y_{i}=\lambda$, then take $\alpha_{i}^{i}=B_{i}$,

(b) $x_{i} \neq \lambda, y_{i}=\lambda$, then we may assume there is a production $S \rightarrow x S$ in $P, x \neq \lambda$. Take $\alpha_{j}^{\prime}=\bar{B}_{i}$, where $\bar{B}_{i} \Rightarrow{ }^{+} y B_{i}, y$ in $\tau\left(x_{i}\right)$, via interpretations of $S \rightarrow x S$,

(c) $x_{i}=\lambda, y_{i} \neq \lambda$, similar to (b),

(d) $x_{i} \neq \lambda \neq y_{i}$, then we may assume there is a production $S \rightarrow x S y$ in $P$, with $x \neq \lambda$ and $y \neq \lambda$. Take $\alpha_{1}^{i}=\bar{B}_{i}$, where $\tilde{B}_{i} \Rightarrow x^{\prime} B_{i} y^{\prime}, x^{\prime}$ in $\tau\left(x_{i}\right), y^{\prime}$ in $\tau\left(y_{j}\right)$, via interpretations of $S \rightarrow x S y$. In all cases we have replaced $A \rightarrow \alpha_{1} \ldots \alpha_{m}$ by $A \rightarrow \alpha_{1}^{1} \cdots \alpha_{m}^{\prime}$, where the $\alpha_{i}^{t}$ are new nonterminals equal to either $B_{i}, \bar{B}_{j}$ or the sentence symbol of the sub-grammar generating $\tau\left(x_{i}\right) \tau\left(L\left(B_{i}, \Rightarrow\right)\right) \tau\left(y_{i}\right)$.

Thus the accumulated productions form $P_{\tau}$, and $\mu_{\tau}$ is defined by the construction, hence $G_{\tau} \vec{g} G\left(\mu_{\tau}\right)$ and $L\left(G_{\tau}, \Rightarrow\right)=\tau\left(L\left(G^{\prime}, \Rightarrow\right)\right)$ as desired completing the theorem.

\section{II.4.3 Characterization Theorems}

It is the aim of this section to demonstrate three results. First we establish that every infinite grammar form generates a full principal semi-AFL under g-interpretation. This enables us to exhibit many sub-context-free families which are not g-gramatical. Second we show that the g-grammatical family of every infinite grammar form is also an s-grammatical family. Hence the only family not generated under $s$-interpretations is $\mathcal{L}$ (FIN). Since there are s-grammatical families which are not g-grammatical this reinforces our earlier remark that s-interpretation is more general than g-interpretation. Third, we consider when $\mathcal{L}_{s}(G, \Rightarrow)$ equals $\mathcal{L}$ (REG) or $\mathcal{L}$ (LIN). 
We now turn to our first result. Letting $L$ be in $\mathcal{L}_{g}(G, \Rightarrow)$ for some infinite grammar form $G$, it follows that $\rho(L) \subseteq \mathcal{L}_{\mathrm{g}}(G, \Rightarrow)$ where $\mathcal{\delta}(L)$ is the smallest full semi-AFL containing $L$. When equality occurs we say $L$ is a full generator for $\mathcal{L}_{g}(G, \Rightarrow)$.

It is easy to see that under g-interpretations changing an appearance of a terminal symbol into an appearance of a new terminal symbol does not affect the generative capacity of a grammar form. Likewise changing a nonempty terminal word into a single terminal symbol on the right hand side of some production does not affect the generative capacity. Similarly in an s-reduced non-expansive grammar form $G=(V, \Sigma, P, S)$ given a production $S \rightarrow \alpha$ we can assume that only the following possibilities occur for $S$-productions:

(i) $\alpha$ is in $\Sigma \cup\{\lambda\}$

(ii) $\alpha$ is in $(\Sigma \cup\{\lambda\})\{S\}(\Sigma \cup\{\lambda\})$

(iii) $\alpha=A_{1} \ldots A_{m} S a, m>0$ where all the $A_{j}$ are nonterminals different from $S$ and $a$ is terminal,

(iv) $\alpha=a S B_{1} \ldots B_{n}, n>0$, where a 11 the $B_{j}$ are nonterminals different from $S$ and $a$ is terminal,

(v) $\alpha=A_{1} \ldots A_{m} S B_{1} \ldots B_{n}, m+n>0$ where all $A_{j}$ and $B_{j}$ are nonterminals different from $S$, and

(vi) $\alpha=A_{1} \ldots A_{m}, m>0$ where the $A_{i}$ are nonterminals different from $S$.

Now each nonterminal different from $S$ defines a sub-grammar form which generates a full semi-AFL (compare Theorem 4.15). Hence in an S-production containing such a nonterminal, $X$ say, $X$ can be replaced by $a x$, $x b$ or $a x b$ where $a$ and $b$ are terminals without any increase in the generative capacity of $G$. This gives the following proposition.

\section{Proposition 4.16}

Let $G=(V, \Sigma, P, S)$ be an infinite $s$-reduced non-expansive grammar form with at least two nonterminals. Then there exists a g-form equivalent grammar form $H=\left(V_{H}, \Sigma_{H}, P_{H}, S\right)$ which is also infinite, s-reduced, non-expansive and satisfies the following conditions:

(a) There exists disjoint terminal alphabets $\Sigma_{a}, \Sigma_{b}$ and $\Sigma_{c}$ such that $\Sigma_{H}=\Sigma \cup \Sigma_{\mathrm{a}} \cup \Sigma_{\mathrm{b}} \cup \Sigma_{\mathrm{c}}$.

(b) $V_{H}=\Sigma_{H} \cup(V-\Sigma)$.

(c) Each production $S \rightarrow x$ in $P_{H}$ with $x$ in $\Sigma_{H}^{*}$ has $x$ in $\Sigma_{c} u\{\lambda\}$.

(d) Each symbol in $\Sigma_{a} \cup \Sigma_{b} \cup \Sigma_{c}$ occurs in one and only one production in $P_{H}$ and there only once. 
(e) Each production $S \rightarrow a S B$ in $P_{H}$ satisfies $\alpha$ is in $\Sigma_{a} U\left(\Sigma_{a}(V-\Sigma)\right)^{*}$ and $\beta$ is in $\Sigma_{b} \cup\left(\Sigma_{b}(V-\Sigma)\right) *$.

(f) Each production $S \rightarrow \alpha$ in $P_{H}$, where $S$ is not in a satisfies $\alpha$ is in $\left(\Sigma_{C}(V-\Sigma)\right)^{+}$.

(g) All productions for all nonterminals different from $S$ are taken unchanged into $\mathrm{P}_{\mathrm{H}}$.

Essentially the terminals from $\Sigma_{c}$ are used to mark the terminating (with respect to $S$ ) $S$-productions and each of the terminals in $\Sigma_{a} \cup \Sigma_{b} \cup \Sigma_{c}$ is used to either uniquely mark the appearance of a nonterminal different from $s$ or the absence of such nonterminals.

We now state and prove our main theorem.

\section{Theorem 4.17}

Let $G=(V, \Sigma, P, S)$ be an infinite grammar form. Then $\mathcal{L}_{g}(G, \Rightarrow)$ is a full principal semi-AFL and furthermore a full generator for $\mathcal{L}_{g}(G, \Rightarrow)$ can be effectively constructed from $G$.

Proof: We may assume that $G$ is an $s$-reduced grammar form without any loss of generality. Now if $G$ is expansive $\mathcal{L}_{g}(G, \Rightarrow)=\mathcal{L}(C F)$ and hence $\mathcal{L}_{g}(G, \Rightarrow)$ is a full principal semi-AFL. Full generators for $\mathcal{L}$ (CF) are well known and an interpretation $G^{\prime}$ of $G$ can easily be constructed, which defines such a generator.

Having solved the case of $G$ being expansive assume from now on that $G$ is non-expansive.

Case 1: $S$ is the only nonterminal. Then $\mathcal{L}_{g}(G, \Rightarrow)=\mathcal{L}$ (REG) or $\mathcal{L}(\mathrm{LIN})$. Both $\mathcal{L}(R E G)$ and $\mathcal{L}$ (LIN) are full principal semi-AFLs and full generators for them are well known. Hence we are left with:

Case 2: $V-\Sigma$ contains at least two nonterminals. We proceed by induction on the number of nonterminals. The basis $\#(V-\Sigma)=1$ is subsumed under Case 1. Hence assume the theorem is true for all $G$ with $\#(V-\Sigma) \leq k-1$, for some $k>1$. Consider the case that $G$ has $k$ nonterminals.

Recalling that $f(L)$ is the smallest full semi-AFL generated by $L$, it suffices to show that:

(*) there exists a language $L$ in $\mathcal{L}_{g}(G, \Rightarrow)$ such that $\mathcal{Z}_{g}(G, \Rightarrow) \subseteq \mathcal{f}(L)$.

Moreover this can be further reduced to showing that

$(* *)$ there exists a language $L$ in $\mathcal{L}_{S}(G, \Rightarrow)$ such that 


$$
\begin{aligned}
& \mathcal{L}_{s}(G, \Rightarrow) \subseteq \mathcal{J}(L), \text { since this implies that } \\
& \text { HL }\left(\mathcal{L}_{s}(G, \Rightarrow)\right)=\mathcal{L}_{g}(G, \Rightarrow) \subseteq \mathcal{W}(\mathcal{J}(L))=\mathscr{J}(L) .
\end{aligned}
$$

Let us assume that $G$ satisfies the conditions for $H$ in Proposition 4.16, not only for $S$ but also for each nonterminal. In other words each terminal symbol in $\Sigma$ appears once and only once in some production in $\mathrm{P}$ and moreover we can split $\Sigma$ into three disjoint subsets, namely those terminals used in "terminating" productions, those deposited to the left by each recursive nonterminal and those deposited to the right. We say a production $A \rightarrow \alpha$ for a nonterminal is terminating, for the purpose of this proof, if a does not contain $A$. That we can assume $G$ to be in this "terminal-distinct" form follows from the observation that each $A \neq S$ defines a subgrammar form which satisfies the conditions of Proposition 4.17.

Without loss of generality assume $A \rightarrow \lambda$ is in $P$ for each $A$ in $V-\Sigma$

Letting the left, middle and right terminals be denoted by $\Sigma_{\ell}, \Sigma_{m}$ and $\Sigma_{r}$ where $\Sigma=\Sigma_{\ell} \cup \Sigma_{m} \cup \Sigma_{r}$ we add to $G$ :

(i) the terminals $\bar{\Sigma}_{\ell}=\left\{\bar{a}:\right.$ a in $\left.\Sigma_{\ell}\right\}$ and $\bar{\Sigma}_{r}=\left\{\bar{a}:\right.$ a in $\left.\Sigma_{r}\right\}$ giving $\bar{\Sigma}=\Sigma \cup \bar{\Sigma}_{\ell} \cup \bar{\Sigma}_{r}$, and

(ii) the productions $A \rightarrow \bar{\alpha} A \bar{B}$ for all $A \rightarrow \alpha A B$ in $P$ for all $A$ in $V-\Sigma$, where $\bar{\alpha}(\bar{\beta})$ is $\alpha(B)$ with each left and right terminal replaced by its "barred" version.

Let the resulting grammar form be $\bar{G}=(\bar{V}, \bar{\Sigma}, \bar{P}, \bar{S})$, where $\bar{V}=(V-\Sigma) \cup \bar{\Sigma}$. Clearly $\bar{G} \underset{S}{S}$ and also $G \underset{S}{S} \bar{G}$, hence $G$ and $\bar{G}$ are $s$-form equivalent (in fact strong $s$-form equivalent).

We claim that $L(\bar{G}, \Rightarrow)$ is a full generator for $\mathcal{L}_{g}(G, \Rightarrow)$.

To show this consider an arbitrary interpretation

$G^{\prime}=\left(V^{\prime}, \Sigma^{\prime}, P^{\prime}, S^{\prime}\right) \rightarrow \vec{S} G(\mu)$. We need to prove that $L\left(G^{\prime}, \Rightarrow\right)$ is in $\mathcal{S}(L(\bar{G}, \Rightarrow))$. We do this by sketching the construction of an a-transducer $M_{G^{\prime}}$ which satisfies $L\left(G^{\prime}, \Rightarrow\right)=M_{G^{\prime}}(L(\bar{G}, \Rightarrow))$.

Let $\rightarrow A^{\prime} \rightarrow$ represent an a-transducer with a single input state $\sigma\left(A^{\prime}\right)$ and a single accepting state $\sigma_{a}\left(A^{\prime}\right)$ (this is no loss of generality).

First observe that for each $A$ in $V-(\Sigma \cup\{S\})$ by the inductive assumption $L\left(\bar{G}_{A} \Rightarrow\right)$ is a full generator for $\mathcal{L}_{g}\left(G_{A} \Rightarrow\right)$. Although this has not been proved directly under Case 1, the proof sketch we now outline can be adapted for this purpose. Hence for each $A^{\prime}$ in $V^{\prime}-\Sigma^{\prime}$ with $A^{\prime}$ in $\mu(A), A \neq S$ there is an a-transducer $M_{G^{\prime}} A^{\prime}$ 
such that $L\left(G^{\prime} A^{\prime}, \Rightarrow\right)=M_{G^{\prime}}\left(L\left(\bar{G}_{A}, \Rightarrow\right)\right)$. The construction we now give for $M_{G}$ is inductive and whenever we refer to a copy of the a-transducer for an $A^{\prime}, A^{\prime}$ not in $\mu(S)$, we assume that its state set is unique. To construct $M_{G}$ ' we only consider the $S^{\prime \prime}$-productions of $G^{\prime}$ for all $S^{\prime \prime}$ in $\mu(S)$. Assume initially the existence of an initial state $\sigma$ and an accepting state $\sigma_{a}$ in $M_{G}{ }^{\prime}$. Let the left, middle and right terminal symbols for the $s$-productions be $\Sigma_{a} \cup \bar{\Sigma}_{a}, \Sigma_{c}$ and $\bar{\Sigma}_{b} \cup \bar{\Sigma}_{b}$ respectively. Enumerate the images of the $s$-productions from $1,2, \ldots, n_{S}$ say.

For each production $j: S^{\prime \prime} \rightarrow \alpha$ in $P^{\prime}$, where $S^{\prime \prime}$ is in $\mu(S)$ and $1 \leq j \leq n_{S}$, add the following transitions to $M_{G^{\prime}}$ :

(i) if $\alpha=c^{\prime}$, then $\left(\sigma, c, c^{\prime}, \sigma_{a}\right)$, where $S \rightarrow c$ is in $\bar{P}$.

(ii) if $\alpha=a_{1}^{1} A_{1}^{\prime} \ldots a_{m}^{\prime} A_{m}^{\prime} s^{\prime \prime} b_{j}^{\prime} B_{j}^{\prime} \ldots b_{n}^{\prime} B_{n}^{\prime}$ where $m, n \geq 0, a_{p}$, $b_{q}$ are in $\Sigma^{\prime}$ and the $A_{p}^{\prime}$ and $B_{q}^{\prime}$ are nonterminals then there exists a production $S \rightarrow a_{1} A_{1} \ldots a_{m} A_{m} S_{1} \ldots b_{n} B_{n}$ in $\bar{p}$ and also the production $s \rightarrow \bar{a}_{1} \ldots \bar{a}_{m} A_{m} s \bar{b}_{1} \ldots \bar{b}_{n} B_{n}$.

Since $A \rightarrow \lambda$ is in $\bar{P}$ for each $A$ in $\bar{V}-\bar{\Sigma}$, we can encode the production $j: S^{\prime \prime} \rightarrow \alpha$ with the following transitions: $\left(\sigma, \bar{a}_{1} \ldots \bar{a}_{m}\left(a_{1} \ldots a_{m}\right)^{j} \bar{a}_{1}, a_{j}, \sigma\left(A_{j}^{\prime}\right)\right.$, $\left(\sigma_{a}\left(A_{l}^{\prime}\right), \bar{a}_{2}, a_{2}^{1}, \sigma\left(A_{2}^{\prime}\right)\right)$,

and

$\left(\sigma_{a}\left(A_{m-1}^{\prime}\right), \bar{a}_{m}, a_{m}^{\prime}, \sigma\left(A_{m}^{\prime}\right)\right)$

$\left(\sigma_{a}\left(A_{m}^{\prime}\right), \lambda, \lambda, \sigma\right)$

$$
\begin{aligned}
& \left(\sigma, \bar{b}_{1} \ldots \bar{b}_{n}\left(b_{1} \ldots b_{n}\right)^{j} \bar{b}_{1}, b_{j}^{\prime}, \sigma\left(B_{1}^{j}\right)\right), \\
& \cdot \\
& \left(\sigma_{a}\left(B_{n-1}^{\prime}\right), \bar{b}_{n}, b_{n}^{\prime}, \sigma\left(B_{n}^{\prime}\right)\right) \\
& \left(\sigma_{a}\left(B_{n}^{\prime}\right), \lambda, \lambda, \sigma\right), \\
& \left(\sigma_{a}\left(B_{n}^{\prime}\right), \lambda, \lambda, \sigma_{a}\right) .
\end{aligned}
$$

Because of the structure of $\bar{G}$ a word $u$ derived from $a_{j}^{\prime} A_{1}^{1} \ldots a_{m}^{\prime} A_{m}^{\prime}$ will be output by $M_{G}$ iff a word v derived from $b_{j}^{\prime} B_{1}^{\prime} \ldots b_{n}^{\prime} B_{n}^{\prime}$ is output by $M_{G}$. Moreover this will only occur when there is a derivation $S^{\prime} \Rightarrow{ }^{+} S^{\prime \prime} y \Rightarrow x a y \Rightarrow^{+} x u S^{\prime \prime} v y$ in $G^{\prime}$.

The encoding technique is crucial to the construction, since otherwise $M_{G}$, will create erroneous output. 
( $\left.i i_{i}\right) \quad a=a^{\prime} S^{\prime \prime}, a^{\prime} S^{\prime \prime} b^{\prime}, S^{\prime \prime} b^{\prime}, a^{\prime} S^{\prime \prime} b_{1}^{\prime} B_{j}^{\prime} \ldots b_{n}^{\prime} B_{n}^{\prime}$,

$a_{j}^{\prime} A_{j}^{\prime} \ldots a_{m}^{\prime} A_{m}^{\prime} S^{\prime \prime} b^{\prime}$ or $\alpha=c_{j}^{\prime} C_{j}^{\prime} \ldots c_{r}^{\prime} C_{r}^{\prime}$.

Each of these is dealt with in a similar way to case (ii).

Finally add the transitions $(\sigma a, \lambda, \lambda, \sigma)$ and $\left(\sigma, \lambda, \lambda, \sigma_{a}\right)$ to $M_{G}$. Note that not all words in $L\left(G^{\prime} \Rightarrow\right)$ are accepted by $M_{G^{\prime}}$ and secondly note that there is a $\lambda$-transition (outputting $\lambda$ ) not only from $\sigma_{a}$ to $\sigma$ but also from $\sigma$ to $\sigma_{a}$, and this is true for each a-transducer corresponding to each subgrammar of $G^{\prime}$.

consider each word $w$ in $L\left(G^{\prime}, \Rightarrow\right)$. There is a derivation $S^{\prime} \Rightarrow^{+} W^{\prime} \Rightarrow^{*} w$ in $G^{\prime}$

such that $S^{\prime}$ derives $W^{\prime}$ using only images of $S$-productions and further $W^{\prime}$ does not contain any image of $S$. There is a corresponding derivation $S \Rightarrow^{+} x$ in $\bar{G}$ of which $S^{\prime} \Rightarrow^{+} W^{\prime}$ is the image. Since $S^{\prime} \Rightarrow^{+} W^{\prime}$ can only contain the image of exactly one terminating s-production, the other productions used are images of recursive S-productions. Now the encoding suggested above results in using some multiple of each recursive $S$-production, rather than the original single applications. This clearly can always be carried out and each word of $L(\bar{G}, \Rightarrow)$ either gives rise to a unique decoding into a word of $L^{\prime}\left(G^{\prime}, \Rightarrow\right)$ or it cannot be decoded. In the former case $M_{G}$, accepts the word and gives the correct output and in the latter case $M_{G}$, rejects the word.

This completes the proof sketch that for $G^{\prime \prime}$ an arbitrary s-interpretation of $G, L\left(G^{\prime}, \Rightarrow\right)$ is in $\mathcal{I}\left(L\left(G^{\prime}, \Rightarrow\right)\right)$.

As immediate applications of this Theorem we have:

\section{Corotzary 4.18}

The families of metalinear, nonterminal bounded and derivation bounded languages are not g-grammatical families.

We now consider our second result namely the comparison of the collections of g-grammatical and s-grammatical families. By Lemma 2.8 and Theorem 2.5 we know that $\mathcal{L}$ (FIN) is both a g-grammatical family and not an s-grammatical one. Since the only other g-grammatical family "below" $\mathcal{Z}$ (FIN) is $\{0\}$, which is also s-grammatical, we turn our attention to g-grammatical families generated by infinite grammar forms. 


\section{Theorem 4.19}

Let $G=(V, \Sigma, P, S)$ be an infinite grammar form. Then $\mathcal{L}_{g}(G, \Rightarrow)$ is $s$-grammatical.

Proof: The basic idea of the proof is to construct a grammar from $H$ from $G$ such that $H$ and $G$ are $g$-form equivalent and $\mathcal{L}_{5}(H, \Rightarrow)=\mathcal{Z}_{g}(H, \Rightarrow)$. Without loss of generality we can assume $\Sigma=\{a\}$ and hence $\mathcal{L}_{s}(G, \Rightarrow)$ is closed under union and intersection with regular sets. Let $H=\left(V_{H},\{a\}, P_{H}, S\right)$ where $V_{H}=V \cup\{A]$, where $A$ is some new nontermina $]$ and $P_{H}$ contains the productions of $P$ modified by replacing each appearance of a with an $A$. Finally the productions $A \rightarrow \lambda$ and $A \rightarrow a A$ are added to $P_{H}$. By simulation $\mathcal{L}_{S}(G, \Rightarrow) \subseteq \mathcal{Z}_{S}(H, \Rightarrow)$ and $\mathcal{L}_{g}(G, \Rightarrow) \subseteq \mathcal{Z}_{g}(H, \Rightarrow)$. We now show that for each $H^{\prime}=\left(V_{H}^{\prime}, \Sigma^{\prime}, P_{H}^{\prime}, S^{\prime}\right) \Delta \Delta_{S}(H)$ there is a $G^{\prime}=\left(V^{\prime}, \Sigma^{\prime \prime}, P^{\prime}, S^{\prime}\right) \underset{S}{Q} G\left(\mu^{\prime}\right)$ and a regular substitution $\tau$ such that $L\left(H^{\prime} \Rightarrow\right)=\tau\left(L\left(G^{\prime}, \Rightarrow\right)\right)$; In other words $\mathcal{L}_{s}(H, \Rightarrow) \subseteq \mathcal{Z}_{g}(G, \Rightarrow)$ which shows that $\mathscr{L}_{\mathrm{g}}(H, \Rightarrow)=\mathcal{X}_{\mathrm{g}}(\mathrm{G}, \Rightarrow)$.

Consider such an $H^{\prime}$. Essentially we let $G^{\prime}$ consist of the G-portion of $H^{\prime}$ together with a different terminal symbol for each image of $A$. Let $\Sigma^{\prime \prime}=\left\{\left[A^{\prime}\right]: A^{\prime}\right.$ is in $\left.\mu(A)\right\}$ and $V^{\prime}=V_{H}^{\prime}-\left(\mu(A) \cup \Sigma^{\prime}\right) \cup \Sigma^{\prime \prime}$. For each production $B \rightarrow \alpha$ in ${ }_{H}^{\prime}$ where $B$ is not in $\mu(A)$ take the production $B \rightarrow[\alpha]$ into $P^{\prime}$ where $[\alpha]$ denotes $\alpha$ with each image $A^{\prime}$ of A replaced by $\left[A^{\prime}\right]$.

For each $A^{\prime}$ in $\mu(A)$ the language $\left\{x: A^{\prime} \Rightarrow^{*} x\right.$ in $\left.H^{\prime}\right\}$ is clearly reguiar, since it is obtained from interpretations of $A \rightarrow$ a and $A \rightarrow \lambda$. Let this be $\tau\left(\left[A^{\prime}\right]\right)$ for each $A^{\prime}$ in $\mu(A)$. By construction it should be clear that $L\left(H^{3} \Rightarrow\right)=\tau\left(L\left(G^{\prime}, \Rightarrow\right)\right)$, hence $\mathcal{K}_{\mathrm{g}}(G, \Rightarrow)=\mathcal{L}_{\mathrm{g}}(H, \Rightarrow)$.

It remains to prove that $\mathcal{L}_{s}(H, \Rightarrow)=\alpha_{g}(H, \Rightarrow)$. It is sufficient to show that $\mathcal{L}_{\mathrm{S}}\left(\mathcal{L}_{\mathrm{s}}(H, \Rightarrow)\right) \subseteq \mathcal{K}_{\mathrm{S}}(H, \Rightarrow)$. Let $H^{\prime}=\left(V_{H}^{\prime}, \Sigma^{3}, P_{H}^{\prime}, S^{\prime}\right) \underset{S}{\Delta} H(\mu)$ and $h: \Sigma^{*} \rightarrow \Sigma^{\prime \prime}$ be a homomorphism. We construct an $H^{\prime \prime} \nabla_{S} H\left(\mu^{\prime}\right)$ such that $L\left(H^{\prime \prime}, \Rightarrow\right)=h\left(L\left(H^{\prime}, \Rightarrow\right)\right)$.

Let $\mu(A)=\left\{A_{1}, \ldots, A_{m}\right\}, m \geq 1$ and $\Sigma^{\prime}=\left\{a_{1}, \ldots, a_{n}\right\}, n \geq 1$. Let $h\left(a_{n}\right)=b_{1} \ldots b_{n_{1}}, n_{1} \geq 0, b_{i}$ in $\Sigma^{*}$. The terminal $a_{n}$ appears in at most two kinds of productions, either $A_{i} \rightarrow a_{n}$ or $A_{j} \rightarrow a_{n} A_{j}$, for some $j$ and $j$.

(i) Replace each production of the form $A_{j} \rightarrow a_{n}$ by either $A_{i} \rightarrow \lambda$ if $n_{1}=0$ or $A_{i} \rightarrow b_{1} B_{i, 1}, B_{i, 1} \rightarrow b_{2} B_{i, 2}, \ldots, B_{i, n_{1}-1} \rightarrow b_{n_{1}}$, where the $B_{i, k}$ are new nonterminals, otherwise.

(ii) For each production of the form $A_{i} \rightarrow a_{n} A_{j}$ either remove it and replace $A_{j}$ everywhere by $A_{j}$ if $n_{1}=0$ or replace it by $A_{i} \rightarrow b_{1} B_{i, j, 1}, B_{i, j, 1} \rightarrow b_{2} B_{i, j, 2}, \ldots, B_{i, j, n_{1}-1} \rightarrow b_{n_{1}} A_{j}$, where the $B_{i, j, k}$ are new nonterminals, otherwise. 
Let the resulting grammar form be $H_{1}$, then we claim that $L\left(H_{1}, \Rightarrow\right)=h_{1}\left(L\left(H^{\prime}, \Rightarrow\right)\right)$ where $h_{j}\left(a_{i}\right)=a_{i}, 1 \leq i<n$ and $h_{1}\left(a_{n}\right)=h\left(a_{n}\right)$. If $h\left(a_{n}\right)=\lambda$ then we have indeed erased $a_{n}$ in $L\left(H^{\prime}, \Rightarrow\right)$ to give $L\left(H_{1}, \Rightarrow\right)$, otherwise we have included unique productions to generate $h\left(a_{n}\right)$ rather than $a_{n}$ at each position that $a_{n}$ would have been generated in $H^{\prime}$. Hence $L\left(H_{1}, \Rightarrow\right)=h_{1}\left(L\left(H^{\prime}, \Rightarrow\right)\right)$. Assuming without loss of generality that $\Sigma^{\prime} \cap \Sigma^{\prime \prime}=\emptyset$ we can iterate the construction to obtain $H^{\prime \prime}$ such that $L\left(H^{\prime \prime}, \Rightarrow\right)=h\left(L\left(H^{\prime}, \Rightarrow\right)\right)$ obtaining the desired result.

We have therefore demonstrated that each g-grammatical family apart from $\mathcal{L}$ (FIN) is also s-grammatical.

To close this section we consider our final area for discussion, that is when does $\mathcal{L}_{s}(G, \Rightarrow)=\mathcal{L}(R E G)$ or $\mathcal{L}$ (LIN)? We first have:

\section{Theorem 4.20}

Let $G=(V, \Sigma, P, S)$ be a grammar form. Then $\mathcal{L}_{s}(G, \Rightarrow) \geq \mathcal{L}(R E G)$ iff there is an a in $\Sigma$ such that $a^{*} \subseteq L(G, \Rightarrow)$.

Proof: if: Let $R \subseteq \Delta^{*}$ be an arbitrary regular language. We show that $R$ is in $\mathcal{L}_{S}(G, \Rightarrow)$. Since $a^{*} \subseteq L(G, \Rightarrow)$ for some a in $\Sigma$, there exists $G_{1} \& G_{\text {with }} L\left(G_{1}, \Rightarrow\right)=a^{*}$, since $\mathcal{L}_{s}(G, \Rightarrow)$ is closed under intersection with regular sets by Theorem 4.13 . Since $a^{*}$ is in $\mathcal{L}_{S}(G, \Rightarrow), \Delta^{*}$ is in $\mathcal{Z}_{S}(G, \Rightarrow)$ since $s$-grammatical families are closed under dfl-substitution (Theorem 4.13). Finally, since $R=\Delta^{*} n R$, then $R$ is in $\mathcal{L}_{S}(G, \Rightarrow)$ by Theorem 4.13 .

only if: Since $\mathcal{L}_{s}(G, \Rightarrow) \geq \mathcal{L}(R E G)$, $b^{*}$ must be in $\mathcal{L}_{s}(G, \Rightarrow)$ for some $b$, hence by Lemma 2.1 a* $\subseteq L(G, \Rightarrow)$ for some a in $\Sigma$.

This also yields

\section{Corozzary 4.21}

Let $G$ be a grammar form.

Then $\mathscr{L}_{\mathrm{S}}(\mathrm{G}, \Rightarrow) \geq \mathcal{L}(\mathrm{REG})$ iff $\mathscr{L}_{\mathrm{S}}(\mathrm{G}, \Rightarrow) \supseteq \mathcal{L}(\mathrm{FIN})$.

We can now characterize those grammar forms which give exactly $\mathcal{L}$ (REG) under s-interpretations. 
Let $G=(V, \Sigma, P, S)$ be a reduced grammar form.

Then $\mathcal{L}_{S}(G, \Rightarrow)=\mathcal{L}(R E G)$ iff $(i) G$ is non-self-embedding and (ii) $a^{*} \subseteq L(G, \Rightarrow)$ for some a in $\Sigma$.

Eroof: if: Condition (ii) implies $\mathcal{L}_{S}(G, \Rightarrow) \geq \mathcal{L}$ (REG) and condition (i) implies $\mathcal{L}_{\mathrm{s}}(G, \Rightarrow) \subseteq \mathcal{L}(\mathrm{REG})$.

only if: Since $\mathcal{L}_{S}(G, \Rightarrow) \supseteq \mathcal{L}(R E G)$ condition (ii) must hold and since $\mathcal{Z}_{S}(G, \Rightarrow) \subseteq \mathcal{L}(R E G)$ condition (i) must hold.

We now turn to the question of when $\mathcal{L}_{s}(G, \Rightarrow)=\mathcal{L}(L I N)$. For this case we will not give any proofs, but only the main theorems and appropriate definitions.

We will first consider unary reduced grammar forms.

$A$ nonterminal $A$ in a unary reduced grammar form $G=(V,\{a\}, P, S)$

is left pumping (respectively, right pumping) if for some fixed

$m, n \geq 0$, there are infinitely many values $i$ such that:

$A \Rightarrow a^{i+m_{A a}}{ }^{n}$ (respectively, $A \Rightarrow{ }^{*} a^{m a}{ }^{n+i}$ ).

A nonterminal is pumping if it is both left and right pumping.

Let $A_{1}, \ldots, A_{m}$ be all the pumping nonterminals in $G$. For each $i, 1 \leq i \leq m$, the lengths $j$ of the terminal words $a^{j}$ generated by $A_{i}$ constitute an ultimately periodic set. Denote its period by $p\left(A_{i}\right)$ and let $p$ be the least common multiple of the periods $p\left(A_{1}\right), \ldots, p\left(A_{m}\right)$.

Denote the residue classes modulo $p$ by $R_{0}, R_{1}, \ldots, R_{p-1}$.

We say the residue class $R_{j}$ is $A_{i}$ reachable if there are integers $r, s$ and $t$ such that:

$$
S \Rightarrow a^{r} A_{i} a^{s}, A_{i} \Rightarrow^{*} a^{t+n p} \text {, for a } 11 n \geq 0 \text {, }
$$

where $r+s+t \equiv j$ modulo $p$.

The pumping spectrum of $G$ consists of all integers in al1 $A_{i}$-reachable residue classes, where $1 \leq i \leq m$.

For linear grammar forms we now obtain:

\section{Theorem 4.23}

Let $G=(V,\{a\}, P, S)$ be a reduced unary 1 inear grammar form.

Then $\mathcal{L}_{s}(G, \Rightarrow)=\mathcal{L}$ (LIN) iff (i) $L(G, \Rightarrow)=a$ and (ii) the pumping spectrum of $G$ consists of all non-negative integers. 
This can now be extended to arbitrary grammars by way of the following definition.

Let $G=(V, \Sigma, P, S)$ be a grammar form and a be in $\Sigma$, then $G_{a}$, the a-restriction of $G$, is the subgrammar form $G_{a}=\left(V_{a},\{a\}, P a, S\right)$ of $G$ where $P_{a} \subseteq P$ is the set of all those productions in $P$ which do not contain the letter a.

We now have:

Theorem 4.24

Let $G=(V, \Sigma, P, S)$ be a grammar form.

Then $\mathcal{L}_{S}(G, \Rightarrow)=\mathcal{L}$ (LIN) iff (i) and (ii) hold.

(i) $\mathcal{L}_{s}(G, \Rightarrow) \subseteq \mathcal{L}($ LIN $)$.

(ii) There exists an a in $\Sigma$ such that $a^{*} \leq L(G, \Rightarrow)$ and the pumping spectrum of the reduced a-restriction of $G$ consists of all non-negative integers. 


\section{II.5 Syntax Analysis}

The existence of "universal" parsing algorithms for a given grammar family is the focal point of this section. Given a grammar form $G$, can a parsing algorithm $M$ for the grammar $G$ be supplied such that whenever $G^{\prime} \underset{X}{S} G(\mu)$, for some grammar $G^{\prime}$, a parsing algorithm for $G^{\prime}$ is immediate. In Section 5.1 we examine a technique for s-interpretations, while in Section 5.2 some results on conflict freeness are presented which are a necessary adjunct to a study of precedence parsing for grammar forms. Finally, in Section 5.3 we consider the pushdown acceptor approach, showing that every grammatical family is a pushdown acceptor family and vice versa.

\section{II.5.1 Syntax Analysis of s-grammatical Families}

We are concerned in this section with the following problem:

Given a parsing method $M$ for a grammar $G$ can $M G$ be used as a parsing method for $G^{\prime}$, where $G^{\prime} \vec{x} G(\mu)$ ? If so, then how economic is this indirect parsing technique?

Under s-interpretation we demonstrate that this is indeed the case and, further, when only recognition is required for $G^{\prime}$, the time taken to recognize an arbitrary word $x^{\prime}$ in $\Sigma^{\prime *}$ is of the same order as the time taken to parse words $y,|y|=\left|x^{\prime}\right|$, y in $\Sigma^{*}$, by $M_{G}$.

Under g-interpretation on the other hand the relationship between words in $\Sigma^{\prime *}$ and words in $\Sigma^{*}$ is not as pleasant as for s-interpretations. The set $\left\{x: x^{\prime}\right.$ is in $\left.\mu(x)\right\}$, for arbitrary $x^{\prime}$ in $\Sigma^{\prime} *$ is a singleton set in the case of s-interpretations, whereas in the case of g-interpretations it may be infinite, since erasing and identification is allowed. Therefore in the following attention is restricted to s-interpretation families only.

The central idea is based upon Lemma 2.1 , namely, if a word $x^{\prime}$ over $\Sigma^{\prime}$ is in $L\left(G^{\prime}, \Rightarrow\right)$ then there is a derivation $S \Rightarrow^{+} \mu^{-1}\left(x^{\prime}\right)$ in $G$. of course, the converse does not hold, therefore the major difficulty occurs when $\mu^{-1}\left(x^{\prime}\right)$ is found to be in $L(G, \Rightarrow)$, since it is still necessary to decide whether $x^{\prime}$ is in $L\left(G^{\prime}, \Rightarrow\right)$ or not. However in this case the extra information that $\mu^{-1}\left(x^{\prime}\right)$ is in $L(G, \Rightarrow)$ is sufficient to allow a fast recognition procedure to be carried out on a bracketed version of $X^{\prime}$, based upon the fast recognition of parenthesis languages.

Let $G=(V, \Sigma, P, S)$ be a grammar form and $M_{G}$ a parsing method for $G$, which for each word $x$ in $\Sigma^{*}$ either yields all leftmost derivations of $x$, one after another, if $x$ is in $L(G, \Rightarrow)$ or rejects $x$ if it is not in $L(G, \Rightarrow)$. Let $G^{\prime}=\left(V^{\prime}, \Sigma^{\prime}, P^{\prime}, S^{\prime}\right){ }_{S} G(\mu)$. Then a parsing method $M_{G^{\prime}}$ for $G^{\prime}$ can be obtained as follows. For each word $x^{\prime}$ in $\Sigma^{\prime *}, M_{G^{\prime}}$ 
consists of at most three major steps. These are:

(1) $M_{G^{\prime}}$, determines the unique word $x=\mu^{-1}\left(x^{\prime}\right)$,

(2) $M_{G^{\prime}}$ parses $x$ using $M_{G}$, and

(3) $M_{G^{\prime}}$ checks to see if there are G-derivations of $x$, which via interpretation yield $G^{\prime}$-derivations of $x^{\prime}$.

For the purposes of the algorithm we need to introduce bracketed versions of $G$ and $G^{\prime}$. Assume the elements of $P$ are arbitrarily but uniquely numbered from 1 to \#P. Let $\Delta=\left\{(j,)_{i}: 1 \leq i \leq \# P\right\}$ and 1 et the bracketed version of $G$, $\hat{G}$ be defined by:

$$
\hat{G}=(V \cup \Delta, \Sigma \cup \Delta, \hat{P}, S)
$$

where $\hat{P}=\left\{A+\left({ }_{i} \alpha\right)_{i}: A+\alpha\right.$ is in $P$ and $A \rightarrow \alpha$ is numbered $\left.i\right\}$. The bracketed version of $G^{\prime}, \hat{G} \hat{G}^{\prime}$ is defined as:

$$
\hat{G}^{\prime}=\left(V^{\prime} \cup \Delta, \Sigma^{\prime} \cup \Delta, \hat{P}^{\prime}, S^{\prime}\right)
$$
$\begin{aligned} \text { where } \hat{p}^{\prime}= & \left\{A^{\prime} \rightarrow\left(a_{i}^{\prime}\right)_{i}: A^{\prime} \rightarrow \alpha^{\prime} \text { is in } \mu(A \rightarrow \alpha) \text { for some } A \rightarrow \alpha \text { and }\right. \\ & A \rightarrow \alpha \text { is numbered } i\} .\end{aligned}$

Finally, define $\hat{\mu}$ by $\hat{\mu}(X)=\mu(X)$, for all $x$ in $V, \hat{\mu}\left(l_{j}\right)=l_{i}$ and $\left.\left.\hat{\mu}()_{j}\right)=\right)_{i}, \quad l \leq i \leq \# P . \quad$ Crearly $\hat{G} ! \Delta_{S} \hat{G}(\hat{\mu})$.

We now expand the three steps of $M_{G}$ ' mentioned above.

\section{Step 1:}

$M_{G}$, determines the unique word $x=\mu^{-1}\left(x^{\prime}\right)$. Note that this is a linear (in $\left.\left|x^{1}\right|\right)$ time operation.

\section{Step 2:}

$M_{G}$ ' parses $x$ by $M_{G}$. If $x$ is rejected by $M_{G}$ then $x$ ' is rejected by $M_{G^{\prime}}$, otherwise suppose $M_{G}$ determines that $\delta_{1}, \ldots, \delta_{m}, m>0$, are the leftmost derivations of $x$ in $G$.

\section{Step 3:}

$$
\text { For } \delta:=\delta_{1}, \ldots, \delta \mathrm{m} \text { do }
$$

(a) $M_{G}$, determines the derivation $\hat{\delta}$ obtained by replacing each occurrence of a production $A \rightarrow \alpha$ in $\delta$ by $A \rightarrow\left(i_{i}^{\alpha}\right)_{i}$ from $\hat{P}$. Let $\hat{x}$ denote the bracketed version of $x$ generated by $\hat{\delta}$.

(b) $M_{G^{\prime}}$ determines $\hat{x}^{\prime}$ the bracketed version of $x^{\prime}$ from $\hat{x}$ by consulting $x^{\prime}$. Observe that $x^{\prime}$ is in $L\left(G^{\prime}, \Rightarrow\right)$ iff $\hat{x}^{\prime}$ is in $L\left(\hat{G}^{\prime}, \Rightarrow\right)$.

(c) $M_{G^{\prime}}$ now parses $\hat{x}^{\prime}$ in a bottom-up manner. Furthermore, it simultaneously performs all parses of $\hat{x}^{\prime}$ which conform to the shape of the derivation tree determined by $\hat{\delta}$.

If during these bottom-up reductions the "sentential form" 
$\alpha\left({ }_{i} y_{0} Y_{1} y_{1} \ldots Y_{q}^{y_{q}}\right)_{i}^{\beta}$

occurs, where $q \geq 0, Y_{1}, \ldots, Y_{q}$ are sets of nonterminals, $y_{0}, \ldots, y_{q}$ are terminal words and $\alpha, \beta$ are words over $V^{\prime} u \Delta$, then we obtain

$\alpha \times \beta$

if $x=\left\{A_{0}^{\prime}: A_{0}^{\prime} \rightarrow\left({ }_{i} y_{0} A_{j}^{\prime} y_{1} \ldots A_{q}^{\prime} y_{q}\right)_{i}\right.$ is in $\hat{P}^{\prime}$ and $A_{j}^{\prime}$ is in $y_{j}$, $1 \leq j \leq q\}$.

Clearly $x^{\prime}$ is in $L\left(G^{\prime}, \Rightarrow\right)$ iff $\hat{x}^{\prime}$ determines a set of nonterminals including $S^{\prime}$.

(d) If $S^{\prime}$ is in the set of nonterminals determined by $\hat{x}^{\prime}$, then $M_{G}{ }^{\prime}$ constructs al1 leftmost $G^{\prime}$-derivations from $x^{\prime}$, which conform with $\hat{\delta}$ by repeatedly traversing in a top-down manner the output of (c). At each traversal single nonterminals are chosen from the sets of nonterminals, which conform with those already chosen. If there are at most $k$ nonterminals in each set of nonterminals and the parse tree produced by (c) has $r$ internal nodes, then at most $k^{r}$ distinct derivation trees can be produced by (d).

Let us clarify this algorithm by working through an example.

Exampte 5: Let $G$ be defined by the following numbered productions:

$1: S \rightarrow A B: 2: S \rightarrow C D ; 3: A \rightarrow a A b ; 4: A \rightarrow a b ; 5: B \rightarrow C B$

$6: B \rightarrow c ; 7: C \rightarrow a C ; 8: C \rightarrow a ; 9: D \rightarrow b D C ; 10: D \rightarrow b C$

Let $\Delta=\left\{\left({ }_{j},\right)_{i}: 1 \leq i \leq 10\right\}$ and $G^{\prime} \rightarrow \vec{S} G(\mu)$ be defined by:

$S^{\prime} \rightarrow A^{\prime} B^{\prime} ; S^{\prime} \rightarrow A^{\prime} B^{\prime \prime} ; S^{\prime} \rightarrow C^{\prime} D^{\prime} ; A^{\prime} \rightarrow d A^{\prime \prime} e ; A^{\prime} \rightarrow d f ;$

$A^{\prime \prime} \rightarrow d A^{\prime} e ; A^{\prime \prime} \rightarrow d f ; B^{\prime} \rightarrow g B^{\prime} ; B^{\prime} \rightarrow h ; B^{\prime \prime} \rightarrow h B^{\prime \prime} ;$

$B^{\prime \prime} \rightarrow g B^{\prime} ; C^{\prime} \rightarrow d C^{\prime} ; C^{\prime} \rightarrow d ; D^{\prime} \rightarrow f D^{\prime} h ; D^{\prime} \rightarrow e g^{\prime} ; D^{\prime} \rightarrow f g ;$

where $\mu(S)=S^{\prime}, \mu(A)=\left\{A^{\prime}, A^{\prime \prime}\right\}, \mu(B)=\left\{B^{\prime}, B^{\prime \prime}\right\}, \mu(C)=C^{\prime}$,

$\mu(D)=D^{\prime}, \mu(a)=d, \mu(b)=\{e, f\}$ and $\mu(c)=\{g, h\}$. Now

$L(G, \Rightarrow)=\left\{a^{i} b^{j} c^{j}: i, j \geq 1\right\} \cup\left\{a^{i} b^{j} c^{j}: i, j \geq 1\right\}$, a well known inherently ambiguous language. Note that for each word in $L(G, \Rightarrow)$, $G$ gives at most two distinct derivation trees.

Consider $x^{\prime}=$ ddfegh.

Step 1: $x=\mu^{-1}\left(x^{\prime}\right)=$ a abbcc.

Step 2: $M_{G}$ produces two leftmost derivations for $x$, namely

$\delta_{1}: S \stackrel{L}{\Rightarrow} A B \stackrel{L}{\Rightarrow} a A b B \stackrel{L}{\Rightarrow} a a b b B \stackrel{L}{\Rightarrow} a a b b c B \stackrel{L}{\Rightarrow} a a b b c c$

and

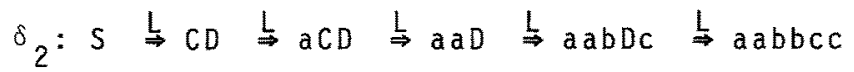

which may be represented as $1,3,4,5,6$ and $2,7,8,9,10$

respectively. 
Step 3: Consider $\delta=\delta_{1}$.

(a) Determine $\hat{\delta}: S \stackrel{L}{\Rightarrow}\left({ }_{1} A B\right)_{1} \stackrel{L}{\Rightarrow}\left({ }_{1}\left(3_{3} a A b\right)_{3} B\right)_{1}$

$\Rightarrow\left({ }_{1}\left(3^{a}\left(4^{a b}\right)_{4}^{b}\right)_{3} B\right)_{1} \stackrel{b}{\Rightarrow}\left({ }_{1}\left(3^{a}\left(4^{a b}\right)_{4} b\right)_{3}\left(5^{c B}\right)_{3}\right)_{1}$

$\stackrel{L}{\Rightarrow}\left({ }_{1}\left({ }_{3}\left({ }_{4} a b\right)_{4} b\right)_{3}\left({ }_{5} c\left({ }_{6} c\right)_{6}\right)_{5}\right)_{1}=\hat{x}$.

(b) Determine $\hat{x}^{\prime}$ from $\hat{x}$.

$\hat{x}^{\prime}=\left({ }_{1}\left({ }_{3} d\left({ }_{4} d f\right)_{4} e\right)_{3}\left({ }_{5} g\left({ }_{6} h\right)_{6}\right)_{5}\right)$

(c) We leave it to the reader to construct $\hat{G}^{\prime}$. Consider the "parse tree" obtained from $\hat{x}^{\prime}$ using $\hat{G}^{\prime}$.

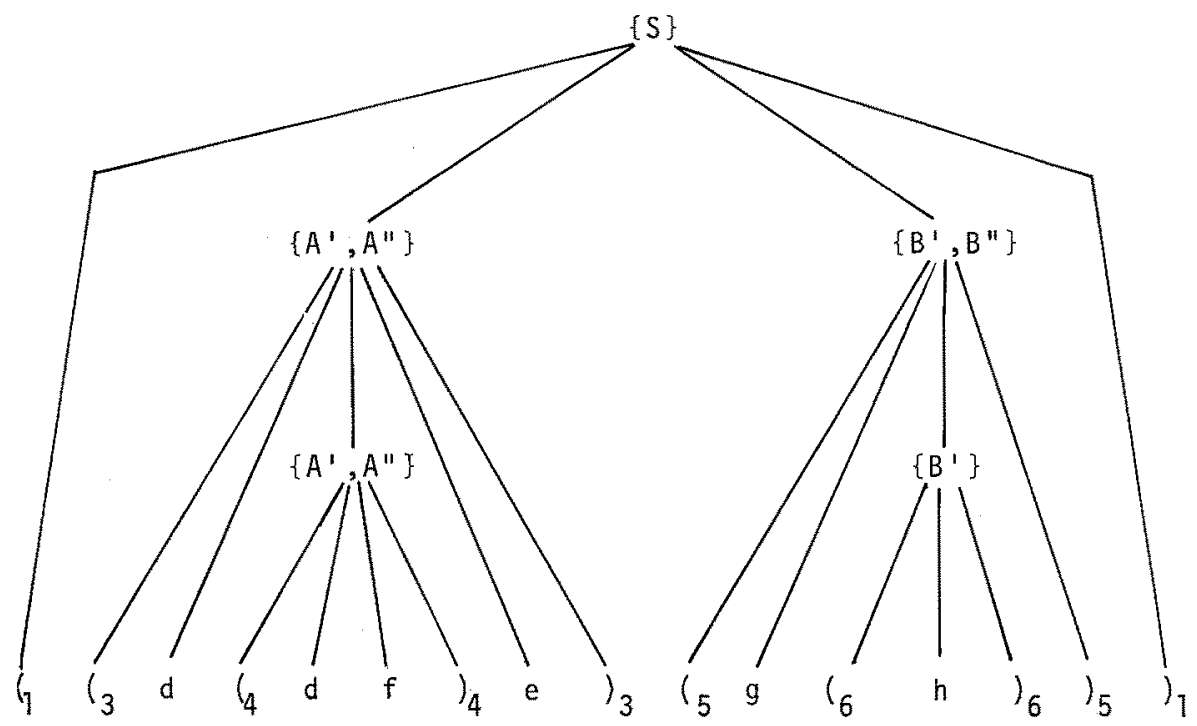

Since $S^{\prime}$ is in the set of nonterminals determined by $\hat{x}^{\prime}, \hat{x}^{\prime}$ is in $L\left(G^{\prime}, \Rightarrow\right)$, that is, $x^{\prime}$ is in $L\left(G^{\prime}, \Rightarrow\right)$.

(d) Construct all possible leftmost derivations in $G^{\prime}$.

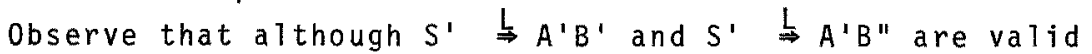
initially, the combinations $S^{\prime} \stackrel{L}{\Rightarrow} A^{\prime \prime} B^{\prime}$ or $S^{\prime} \stackrel{L}{\Rightarrow} A^{\prime \prime} B^{\prime \prime}$ given by the "parse tree" are invalid, since they do not correspond to any productions in $P^{\prime}$. Hence we obtain $S^{\prime} \stackrel{L}{\Rightarrow} A^{\prime} B^{\prime} \stackrel{L}{\Rightarrow} d A^{\prime \prime} e B^{\prime} \stackrel{L}{\Rightarrow} d d f e B^{\prime} \stackrel{L}{\Rightarrow} d d f e g B^{\prime} \stackrel{L}{\Rightarrow}$ ddfegh and

$S^{\prime} \stackrel{L}{\Rightarrow} A^{\prime} B^{\prime \prime} \stackrel{L}{\Rightarrow} A^{\prime \prime} e B^{\prime \prime} \stackrel{L}{\Rightarrow} d d f e B^{\prime \prime} \stackrel{L}{\Rightarrow} d d f e g B^{\prime} \stackrel{L}{\Rightarrow}$ ddfegh We leave to the reader the consideration of $\delta=\delta_{2}$. 
Note that if we merely require the recognition of $x^{3}$ rather than the parsing of $x^{\prime}$, then Step $3(d)$ is unnecessary.

Let us now state and prove the theorems governing the time and space complexity of $M_{G}$, relative to that of ${ }^{{ }_{G}}$.

\section{Theorem 5.1}

Let $G$ be an arbitrary grammar form and suppose that there is a parsing method $M_{G}$ for $G$ and a function $t(n), n \geq 0$, such that for each word $x$ of length $n M_{G}$ outputs all leftmost derivations of $x$ in no more than $t(n)$ steps. Let $G^{\prime} \stackrel{S}{S}_{G(\mu)}$ and $p(n), n \geq 0$, be a function such that for each word $x^{\prime}$ of length $n$ in $L\left(G^{\prime}, \Rightarrow\right)$ there are at most $p(n)$ equally shaped derivations of that word. Then for some constant $c$ and each word $x^{\prime}$ in $\Sigma^{\prime *},\left|x^{\prime}\right|=n, M_{G}$, as defined above, yields either all leftmost derivations of $x^{\prime}$ if $x^{\prime}$ is in $L\left(G^{\prime}, \Rightarrow\right)$ or rejects $x^{\prime}$ if $x^{\prime}$ is not in $L\left(G^{\prime}, \Rightarrow\right)$ in at most $c . t(n), p(n)$ steps.

Proof: The number of steps required by $M_{G^{\prime}}$ for (1) and (2) is $n$ and $t(n)$ respectively. Assuming $x$ is in $L(G, \Rightarrow)$, let $\ell_{\eta}, \ldots, l_{m}$ be the lengths of $\delta_{1}, \ldots, \delta_{m}$ the leftmost derivations of $x$. Since $\delta_{1}, \ldots, \delta_{m}$ are produced in time at most $t(n)$, we have $\sum_{i=1}^{m} l_{i} \leq t(n)$. Consider the number of steps required in (3). Now (3a) involves a single scan of each $\delta_{i}$, each replacement of a production involving $c_{1}$ steps, say. Hence (3a) takes at most $\sum_{i=1}^{m} a_{i} c_{1}$ steps, that is at most $c_{1} \cdot t(n)$ steps. Now $(3 b)$ takes $c_{2} \cdot n$ steps, while in step (3c) each reduction can be carried out in constant time, $c_{3}$ say, since the number of possible "righthand sides" is bounded. Recall that the reduction process introduces sets of nonterminals in general. Clearly there are at most (\#V') $q$ such reductions at each step ( $3 c$ ) where $q$ is the maximum number of nonterminals in the right side of a production. Thus for each $\delta_{i}, \hat{x}^{\prime}$ can be parsed using a number of steps proportional to $\ell_{i}$. Hence the number of steps in $(3 c)$ is at most

$\sum_{i=1}^{m} c_{3} \cdot l_{i} \leq c_{3} t(n)$ steps. Finally in (3d) $M_{G}$, need only traverse the output of (3c) at most $p(n)$ times and the number of steps required for each traversal is proportional to the length of $\delta_{i}$. Step (3d) requires therefore at most $\sum_{i=1}^{m} c_{4} \cdot l_{i} \cdot p(n) \leq c_{4} \cdot p(n) \cdot t(n)$ steps. 
Thus by adding the bounds for steps (1), (2) and (3) we obtain the result.

Eliminating step (3d) from $M_{G}$, we obtain a recognizer for $G^{\prime}$. In this case we have:

\section{Coroltary 5.2}

Let $G$ be an arbitrary grammar form and suppose that there is a parsing method $M_{G}$ for $G$ and a function $t(n), n \geq 0$, such that for each word $x$ of length $n M_{G}$ outputs all leftmost derivations of $x$ in no more than $t(n)$ steps and let $G^{\prime} \rightarrow G(\mu)$. Then for some constant $c$ and each word $x^{\prime}$ in $\Sigma^{\prime}\left|x^{\prime}\right|=n, M_{G^{\prime}}$, as defined above, either accepts $x^{\prime}$ if $x^{\prime}$ is in $L^{\prime}\left(G^{\prime}, \Rightarrow\right)$ or rejects $x^{\prime}$ if $x^{\prime}$ is not in $L^{\prime}\left(G^{\prime}, \Rightarrow\right)$ in at most c. $t(n)$ steps.

In particular if $G$ is unambiguous this corollary indicates that the time taken to recognize whether a word $x^{\prime}$ is in $L^{\prime}\left(G^{\prime}, \Rightarrow\right)$ or not, is of the same order as the time taken to recognize that $\mu^{-1}\left(x^{\prime}\right)$ is or is not in $L(G, \Rightarrow)$.

We now consider the space requirements of $M_{G}$.

\section{Theorem 5.3}

Let $\mathbf{G}=(V, \Sigma, P, S)$ be an arbitrary grammar form. Suppose there exist functions $s(n)$ and $\ell(n), n \geq 0$ and a parsing procedure $M_{G}$ for $G$ with the following properties:

(1) For each word $x$ in $\Sigma^{*}$ and using at most $s(|x|)$ space, $M_{G}$ consecutively determines each leftmost $G$-derivation of $x$ if $x$ is in $L(G, \Rightarrow)$ and rejects $x$ if $x$ is not in $L(G, \Rightarrow)$.

(2) The length of each $G$-derivation of each word $x$ in $L(G, \Rightarrow)$ is at most $\ell(|x|)$.

Then for each $G^{\prime} \underset{S}{S} G(\mu)$ there exists a constant $c$ such that for each word $x^{\prime}$ in $\Sigma^{\prime *}, M_{G^{\prime}}$ using at most $s\left(\left|x^{\prime}\right|\right)+c . l\left(\left|x^{\prime}\right|\right)$ space, yields either a leftmost derivation of $x^{\prime}$ if $x^{\prime}$ is in $L\left(G^{\prime}, \Rightarrow\right)$ or rejects $x^{\prime}$ if $x^{\prime}$ is not in $L\left(G^{\prime}, \Rightarrow\right)$.

Proof: Without loss of generality assume that step (3) is executed for each derivation $\delta_{i}$ of $x$ as soon as $\delta_{i}$ is determined in step (2).

Steps (1) and (2) of $M_{G}$, require $\left|x^{\prime}\right|$ and $s\left(\left|x^{\prime}\right|\right)$ space respectively. Step (3a) requires at most $c_{1} \ell\left(\left|x^{\prime}\right|\right)$ space since each 
derivation step adds exactly one pair of parentheses. Step (3b) can also be carried out in $c_{1} \cdot l\left(\left|x^{\prime}\right|\right)$ space, while step (3c) requires at most $c_{2} \cdot 2\left(\left|x^{\prime}\right|\right)$ space since at each derivation step $M_{G}$ ' has only to record a finite set of nonterminals. Now step (3d) can be carried out in at most $c_{3} \cdot l\left(\left|x^{\prime}\right|\right)$ space, since to generate all possible $G^{\prime}$-derivations of $x^{\prime}, M_{G^{\prime}}$ adds at each derivation step only a finite set of pointers. Finally by adding the above bounds and noting that $\left|x^{\prime}\right| \leq c_{4} \cdot \ell\left(\left|x^{\prime}\right|\right)$ for some $c_{4}$, the upper bound $s\left(\left|x^{\prime}\right|\right)+c \cdot \ell\left(\left|x^{\prime}\right|\right)$

on the space requirement of $M_{G^{\prime}}$ is obtained.

\section{II.5.2 Precedence Relations}

It has already been mentioned in Section 5.1 that under ginterpretations the relationship of a parsing method $M_{G}$ for a master

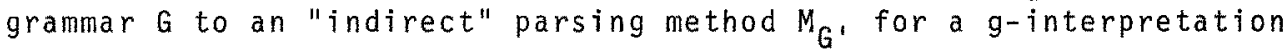
grammar $G^{\prime} \underset{g}{ } G$ is unclear. In this section the property of conflict freeness is considered and it is demonstrated that at least for precedence parsing, onty s-interpretations make sense in the parsing context. We consider non-decreasing and length preserving interpretations as well as $g$ - and s-interpretations.

We first need to define some restricted kinds of g-interpretation.

\section{Definition}

Let $G=(V, \Sigma, P, S), G^{\prime}=\left(V^{\prime}, \Sigma^{\prime}, P^{\prime}, S^{\prime}\right)$ and $G^{\prime} \vec{g} G(\mu)$. Then

(i) $G^{\prime}$ is a nondecreasing interpretation, $G^{\prime} \overrightarrow{n d} G(\mu)$, if for all $X$ in $V$ and for alt $\alpha$ in $\mu(X),|\alpha| \geq 1$.

(ii) $G^{\prime}$ is a length preserving interpretation, $G^{\prime} \overrightarrow{\ell p} G(\mu)$, if $\mu(X) \subseteq V^{\prime}$ for a $11 x$ in $V$.

(iii) $G^{\prime}$ is a unitary interpretation, $G^{\prime} \overrightarrow{u t} G(\mu)$ if $\# \mu(X)=1$ for all $X$ in $V, \mu(A)=A$, for all $A$ in $V-\Sigma$ and $P^{\prime}=\mu(P)$.

(iv) $G^{\prime}$ is a unary interpretation, $G^{\prime} \underset{\text { un }}{\Delta} G(\mu)$ if $G^{\prime} \overrightarrow{a t} G(\mu)$ and $\# \Sigma^{\prime}=1$, that is $G^{\prime}$ is a unary gramar.

(v) $G^{\prime}$ is a unary length preserving interpretation, $G$ ' $\underset{u \neq p}{Q} G(u)$ if it is both a unary and a length preserving interpretation.

If $G$ is a reduced grammar form, then for each a there is exactly one ulp-interpretation with $\Sigma^{\prime}=\{a\}$. Further when $G^{\prime} \underset{\text { Uep }}{\triangle} G$, $G^{\prime}$ is strong form equivalent to $G$.

The notion of unitary interpretation is commoniy encountered in the definition of programming languages. Usually a programming 
language is defined in terms of reference symbols, which in a particular implementation become implementation symbols. Hence the implementation grammar is a unitary interpretation of the reference grammar. This can be seen in ALGOL 60 or PASCAL, where begin, end, if and so on occur in the reference language, and BEGIN, END, If for example, appear in some implementation languages.

We now recall the notions of (Wirth-Weber) precedence relations on context free grammars.

\section{Definition}

Let $G=(V, \Sigma, P, S)$ be a grammar (form). For all $X, Y$ in $V$, write

(i) $X \doteq Y$ if there is a production $A \rightarrow \alpha X Y B$ in $P$, for some $A$ in $V-\sum$ and $\alpha, \beta$ in $V *$.

(ii) $X<Y$ if there is a production $A \rightarrow \alpha X C \beta$ in $P$ and a derivation $C \Rightarrow{ }^{*} Y \gamma$, for some $A, C$ in $V-\Sigma$ and $\alpha, \beta, \gamma$ in $V^{*}$.

(iii) $X \rightarrow Y$ if $Y$ is in $\Sigma$, there is a production $A \rightarrow \alpha B U B$ in $P$, and derivations $B \Rightarrow^{+} \gamma X$ and $U \Rightarrow^{*} Y_{\delta}$, for some $A, B$ in $V-\Sigma, U$ in $V, \alpha, \beta, \gamma, \delta$ in $V^{*}$.

The relations $\doteq,<,>$ are called (Wirth-Weber) precedence relations.

A grammar $G$ is said to be conflict free if at most one precedence relation holds between every ordered pair $(X, Y)$ of symbols in $V$. A collection $\xi$ of grammars is said to be conflict free if each grammar in $\mathcal{E}$ is conflict free.

The importance of this notion of conflict freeness stems from the following definition of a simple precedence grammar. A grammar $G$ is said to be a simple precedence grammar if it is conflict free and uniquely invertible. A grammar $G$ is uniquely invertible if each right hand side of a production in $G$ does not occur as the right hand side of more than one production in $G$.

However in the remainder of this section we only study the relation of conflict free grammar forms to their $x$-interpretation grammars.

We say a grammar is separated if the right hand side of each production is ejther a terminal word or a nonterminal word, but not a mixture. Formally, letting $G=(V, \Sigma, P, S), G$ is separated if for all $A \rightarrow \alpha$ in $P, \alpha$ is in $\Sigma * u(V-\Sigma) *$. 
Theorem 5.4

Let $G=(V, \Sigma, P, S)$ be a grammar form with the property that for each nonterminal $A$ in $V-\Sigma$ there exists a word $x$ in $\Sigma^{+}$such that $A \Rightarrow{ }^{+} x$. Then the following three conditions are equivalent.

(i) $\xi_{g}(G)$ is conflict free.

(ii) $\xi_{n d}(G)$ is conflict free.

(iii) $G$ is separated and whenever a production $A \rightarrow \alpha$ is in $P$ with $\alpha$ in $(V-\Sigma)^{+}$, then $\alpha$ is in $V-\Sigma$.

Proof: Clearly (i) implies (ii).

(ii) implies (iii). Let $x$ be a word in $\Sigma^{+}$such that $S \Rightarrow^{+} x$ in $G$, and let a be some symbol in $x$. Consider $G$ ' a $G(\mu)$ where $G^{\prime}=\left(V^{\prime},\{a\}, P^{\prime}, S\right), \mu(A)=A$ for a $11 \mathrm{~A}$ in $V-\Sigma, \mu(b)=\{a, a a\}$ for a $11 b$ in $\Sigma, P^{\prime}=\mu(P)$ and $V^{\prime}=(V-\Sigma) \cup\{a\}$. Then there is a production $A \rightarrow \alpha a \beta$ in $P^{\prime}$ for some $A$ in $V-\Sigma$ and $\alpha, \beta$ in $V^{\prime *}$. Therefore $a \doteq a$.

Define a homomorphism $h$ on $V *$ by $h(A)=A$ for all $A$ in $V-\Sigma$ and $h(b)=a$ for $a l l b$ in $\Sigma$. We first demonstrate that $G$ is separated. Assume otherwise, in which case there is a production $B \rightarrow \alpha \operatorname{Cb} \beta$ or $B \rightarrow \alpha b C \beta$ in $P$ for some $B, C$ in $V-\Sigma, b$ in $\Sigma$, and $\alpha, \beta$ in $V^{*}$. From the hypothesis there is a derivation $C \Rightarrow y$ for some nonempty terminal word $y$. Then either

$$
B \Rightarrow h(\alpha) \operatorname{Cah}(\beta) \Rightarrow^{+} h(\alpha) a^{|y|} a h(\beta) \text { in } G^{\prime}
$$

or

$$
B \Rightarrow h(\alpha) a C h(\beta) \Rightarrow^{+} h(\alpha) a \text { a }|y| h(\beta) \text { in } G^{\prime} .
$$

Therefore either $a \Rightarrow a$ or $a<$ a also holds in $G^{\prime}$. Hence $G^{\prime}$ has conflicts, a contradiction, therefore $G$ is separated. Now assume there is a production $B \rightarrow \alpha C D \beta$ in $G$, for some $B, C, D$ in $V-\Sigma$ and $\alpha$, $B$ in $V^{*}$. By the hypothesis, there are derivations $C \Rightarrow^{+} x_{1}$ and $D \Rightarrow^{+} x_{2}$ in $G$, where both $x_{1}$ and $x_{2}$ are nonempty. Then

$$
B \stackrel{2}{\Rightarrow} h(\alpha) \operatorname{CDh}(\beta) \Rightarrow^{+} h(\alpha) h\left(x_{1}\right) \operatorname{Dh}(\beta) \Rightarrow^{+} h(\alpha) h\left(x_{1}\right) h\left(x_{2}\right) h(\beta)
$$

in $G^{\prime}$. Since $h\left(x_{i}\right)=a\left|x_{i}\right|, i=1,2$ we have $a \rightarrow a$. Thus $G^{\prime}$ has conflicts, a contradiction, therefore whenever there is a production $x \rightarrow \gamma$ in $P$ with $\gamma$ in $(V-\Sigma)^{+}$, then $\gamma$ is in $V-\Sigma$.

(iii) implies (i)

Assume $(i+i)$ holds. Let $G^{\prime}=\left(V^{\prime}, \Sigma^{\prime}, P^{\prime}, S^{\prime}\right) \Delta G(H)$. By the hypothesis, there are no $A, B$ in $V^{\prime}-\Sigma^{\prime}$ and $a$ in $\Sigma^{\prime}$ such that $A=B$, $a \doteq A$ or $A \doteq a$. However it is possible that $a \doteq b$, for some terminals $a$ and $b$. Now since there are no productions of type $A \rightarrow \alpha$ in $P^{\prime}, \alpha$ in $(V-\Sigma)^{+},|\alpha|>1$, there are no relations $X<Y$ or $X>Y$ for any 
symbols $X, Y$ in $Y^{\prime}$. Therefore $G^{\prime}$ is conflict-free.

Consider the grammar $G$ defined by the productions:

$$
S \rightarrow A B ; A \rightarrow a ; B \rightarrow \lambda \text {. }
$$

Then $\xi_{g}(G)$ is conflict free, but $G$ has a production $C \rightarrow Y$ with $Y$ in $(V-\Sigma)^{2}$. This demonstrates that the condition: for all A in $V-\Sigma$, there is a nonempty terminal word $x$ such that $A \Rightarrow^{+} x$; cannot be removed and still have Theorem 5.4 valid.

Condition ( $i i i)$ in Theorem 5.4 is very strong and implies $G$ has no self-embedding variable. Therefore $\mathcal{L}_{\mathrm{g}}(G, \Rightarrow)=\mathcal{Z}(F I N)$. In fact is can be proved that $\mathcal{L}_{g}(G, \Rightarrow)=\mathcal{L}$ (FIN) if either $\xi_{g}(G)$ or $\xi_{n d}(G)$ is conflict free and $L(G, \Rightarrow)$ contains at least one non-empty word.

Turning to s-interpretations we strengthen our claim that this is the appropriate interpretation mechanism in the parsing context, since the characterization result is particularly simple.

\section{Theorem 5.5}

For each grammar form $G, \xi_{S}(G)$ is conflict free iff $G$ is conflict free.

Proof: Clearly if $\xi_{s}(G)$ is conflict free then $G$ is conflict free, therefore consider the converse.

Let $G^{\prime} \vec{S} G(\mu)$, where $G=(V, \Sigma, P, S)$ and $G^{\prime}=\left(V^{\prime}, \Sigma^{\prime}, P^{\prime}, S^{\prime}\right)$. Now for each word $x^{\prime}$ in $\mu\left(V^{*}\right)$, the set $\mu^{-1}\left(x^{1}\right)=\left\{x: x\right.$ in $V^{*}$ and $x^{1}$ is in $\mu(x)$ \} contains exactly one element. Then for each derivation in $G^{\prime}$, $A^{\prime} \Rightarrow^{+} \alpha^{\prime}$

there is a derivation in $G$

$$
\mu^{-1}\left(A^{\prime}\right) \Rightarrow^{+} \mu^{-1}\left(\alpha^{\prime}\right) \text {. }
$$

It immediately follows that if $X^{\prime} \doteq,<, \rightarrow Y^{\prime}$ in $G^{\prime}$, then $\mu^{-1}\left(X^{\prime}\right) \doteq,<,>\mu^{-1}\left(Y^{\prime}\right)$ in $G$, respectively. Therefore if $G^{\prime}$ has conflicts so does $G$, a contradiction. Hence $\ell_{s}(G)$ is conflict free.

Having demonstrated that s-interpretations preserve conflict freeness we now turn to ep-interpretations.

Theorem 5.6

For each grammar form $G, \xi_{\ell p}(G)$ is conflict free iff there is

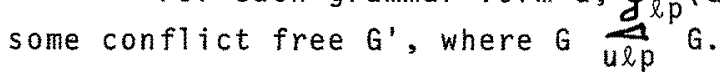


Proof: Clearly if $\xi_{\ell p}(G)$ is conflict free then for all $G$ ' $\overrightarrow{u \ell p} G$, $G^{\prime} \overrightarrow{\ell p} G$ hence $G^{\prime}$ is conflict free. Consider the converse implication. First note that when $G^{\prime} \underset{u l p}{\longrightarrow} G$ then $G \underset{\ell p}{\vec{G}} G^{\prime}$, hence $G^{\prime}$ and $G$ are strongly $\ell p-f o r m$ equivalent. Since $G^{\prime}$ has only one terminal symbol, $\xi_{l p}\left(G^{\prime}\right)=\xi_{s}\left(G^{\prime}\right)$, therefore by Theorem $5.5 \xi_{l p}\left(G^{\prime}\right)$ is conflict free.

It is well known that for each context-free language $L$ there exists a conflict free gramar $G$ with $L(G, \Rightarrow)=L$. We now strengthen this result.

Theorem $5 . ?$

For each context-free language $L$, there exists a grammar form $G$ such that $L=L\left(G, \Rightarrow\right.$ ) and $\xi_{S}(G)$ (and $\xi_{\ell p}(G)$ ) is conflict free.

Proof: Without loss of generality assume $L$ is $\lambda$-free. Now $L=L(F, \Rightarrow)$ for some $\lambda$-free grammar $F$ and there exists a grammar $G$ which fulfills the following conditions:

(i) $L(F, \Rightarrow)=L(G, \Rightarrow)$ and $G$ is obtained effectively from $F$,

(ii) $G$ is conflict free, and

(iii) $G$ only has productions of types $A \rightarrow$ a and $A \rightarrow B C$, and further for each production of type $A \rightarrow B C$, the nonterminal $B$ does not appear in the second position of the right hand side of any production in $G$.

Such a $G$ is constructed in Theorem 5.9. Consider a unary \&pinterpretation $G^{\prime}$ of $G$. Since neither $a \doteq b$ nor $a<b$ are possible in $G$, then $G^{\prime}$ is conflict free. The result follows by Theorems 5.5 and 5.6 .

In the above proof we have used the fact that for each grammar there is an equivalent conflict free grammar. We turn to the consideration of when this result can be generalized for strong $x-$ form equivalence and $x$-form equivalence, $x=g$, nd, \&p and $s$. For strong $x$-form equivalence we give necessary and sufficient conditions, while for $x$-form equivalence we show that every grammar has a g-form equivalent conflict free grammar.

Theorem 5.8

For each grammar form $G$, the following hold:

(1) Let $F$ be the grammar obtained by replacing each occurrence of 
a terminal by a new terminal in some minimal grammar form $G$ ' strongly g-form equivalent to $G$. Then $G$ has a strongly $g$-form equivalent conflict free grammar form iff $F$ is conflict free.

(2) For $x=$ nd or $l p, G$ has a strongly $x$-form equivalent conflict free grammar form iff the grammar form obtained from $G$ by replacing each occurrence of a terminal by a new terminal is conflict free.

(3) G has a strongly s-form equivalent conflict free grammar form iff $G$ is conflict free.

Proof: In all cases the "if" case is obvious, therefore we only consider the "only if" cases in the following.

(1) Suppose $G_{1}=\left(V_{1}, \Sigma_{1}, P_{1}, S_{1}\right)$ is strongly $g$-form equivalent to $G$ and is conflict free. Then by Theorem 3.15 there exists a minimal grammar form $G_{2}=\left(V_{1}, \Sigma_{1}, P_{2}, S_{1}\right)$ strongly g-form equivalent to $G_{1}$ with $P_{2} \subseteq P_{1}$. Since $G_{1}$ is conflict free so is $G_{2}$. Let $G_{3}$ be the grammar form obtained by replacing each occurrence of a terminal by a new terminal in the productions of $G_{2}$. Clearly $G_{3}$ is conflict free, minimal and strongly $g$-form equivalent to $G$. Let $G_{4}$ be the form obtained from $G_{3}$ by deleting all terminals immediately to the right of a terminal in $P_{3}$. Clearly $G_{4}$ is conflict free. Since both $F$ and $G_{4}$ are minimal, symbol tight and strongly g-equivalent they are also pseudo-isomorphic by Theorem 3.18. Consider the following transformation:

(*) Let $\mathrm{H}_{1}$ be a grammar with each occurrence of a terminal a different symbol. Let $\mathrm{H}_{2}$ be obtained from $\mathrm{H}_{1}$ by either replacing one terminal a by either $a b$ or $b, b$ a new terminal or conversely replacing an appearance of $a b$ by $a$.

clearly $\mathrm{H}_{2}$ is conflict free iff $\mathrm{H}_{1}$ is conflict free. Consider $F$ and $G_{4}$. Since they are pseudo-isomorphic there exists a sequence of $(*)$ transformations which applied to $G_{4}$ yields $F$. Hence $F$ is conflict free.

(2) Let $G_{1}=\left(V_{1}, \Sigma_{1}, P_{1}, S_{1}\right)$ be the form obtained from $G$ by replacing each occurrence of a terminal by a new terminal. First consider the length preserving situation. Assume $G$ has some strongly ep-form equivalent conflict free grammar form $G_{2}=\left(V_{2}, \Sigma_{2}, P_{2}, S_{2}\right)$. Because of the transitivity of strong form equivalence we have $G_{1} \overrightarrow{\text { lp }} G_{2}(\mu)$. Let $V_{3}$ be the symbols occurring in the productions of $P_{7}$. Since $\mu$ is length preserving, for each production

$p: x_{0} \rightarrow x_{1} \ldots x_{m}, x_{i}$ in $v_{1}$, 
there is a production, say

$$
h(p): Y_{0} \rightarrow Y_{1} \ldots Y_{m}, Y_{i} \text { in } V_{2} \text {, }
$$

such that $X_{i}$ is in $\mu\left(Y_{i}\right)$ for all $i$. Let $h\left(X_{i}\right)=Y_{i}, 0 \leq i \leq m$. The function $h$ is well defined on $V_{3}$ since (i) for each nonterminal $A$ in $V_{3}$ there is exactly one nonterminal $B$ in $V_{2}$ such that $A$ is in $\mu(B)$, and $(i i)$ each terminal in $V_{3}$ occurs in exactly one production in $P_{1}$ and only once in that production. Hence $h$ is a homomorphism on $V_{3}^{*}$. It is straightforward to verify that if $X \doteq,<,>Y$ then $h(X) \doteq,<,>h(Y)$, for all $X, Y$ in $V_{3}$. Therefore $G_{2}$ has conflicts if $G_{1}$ has conflicts and since $G_{2}$ is conflict free $G_{1}$ must also be conflict free. Second consider the nondecreasing situation. Assume $G$ has a strongly nd-form equivalent conflict free grammar form $G_{2}$. Let $G_{3}$ be the grammar form obtained from $G_{2}$ by replacing each terminal occurrence by a new terminal. As in the length preserving case $G_{3}$ is conflict free and also strongly nd-form equivalent to $G_{1}$. Now in both $G_{3}$ and $G_{1}$ replace terminal subwords in their productions by the leftmost symbol in the terminal subword giving $G_{4}$ and $G_{5}$ respectively. Since this may be accomplished by a sequence of $(*)$-transformations $G_{4}$ is conflict free and $G_{5}$ is conflict free iff $G_{7}$ is conflict free. Clearly $G_{4}$ and $G_{5}$ are strongly nd-form equivalent, moreover they are strongly ep-form equivalent since no production in either $G_{4}$ or $G_{5}$ contains terminal subwords of length greater than one. Hence by the ep case above, $G_{5}$ is conflict free and therefore $G_{1}$ is conflict free.

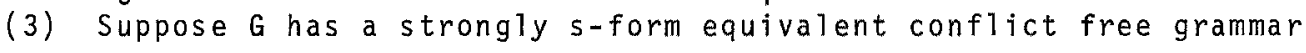
form $G^{\prime}$. By Theorem $5.5 \xi_{S}\left(G^{\prime}\right)$ is conflict free and moreover, since $G$ is in $\xi_{S}\left(G^{\prime}\right), G$ is conflict free.

For the final result of this section we show that each grammar form has a g-form equivalent conflict free grammar form, thereby strengthening a well known result for context free grammars.

\section{Theorem 5.9}

Every grammar form $G=(V, \Sigma, P, S)$ has an $x$-form equivalent conflict free grammar form $F$, where $x=g$, nd, ep or $s$.

Proof: If $L(G, \Rightarrow)=\{\lambda\}$, then let $F=(\{S\}, \emptyset,\{S \rightarrow \lambda\}, S)$. Clearly $F$ is conflict free and $x$-form equivalent to $G$. Consider the case that $G$ is nontrivial. Then by Theorem 4.12 we may assume $G$ is in chomsky Normal Form. Construct $F=\left(V_{F}, \Sigma, P_{F}, S\right)$ as follows: 


$$
\text { Let } \begin{aligned}
V_{F} & =V u\{\hat{A}: A \text { is in } V-\Sigma\}, \text { and } \\
P_{F} & =\{A \rightarrow a, \hat{A} \rightarrow a: A \rightarrow a \text { is in } P \text {, a in } \Sigma\} \\
& \cup\{A \rightarrow \hat{B} C, \hat{A} \rightarrow \hat{B} C: A \rightarrow B C \text { is in } P\} .
\end{aligned}
$$

It is well known that $F$ is conflict free. Since $F \vec{x} G$, immediately $\mathcal{L}_{X}(F, \Rightarrow) \subseteq \mathcal{L}_{X}(G, \Rightarrow)$. Conversely let $G^{\prime}=\left(V^{\prime}, \Sigma^{\prime}, P^{\prime}, S^{\prime}\right) \vec{x} G(\mu)$. For each nonterminal $A$ in $V^{\prime}$, let $\hat{A}$ be a new nonterminal. Define a substitution $\mu^{\prime}$ on $V_{F}$ by $\mu^{\prime}(X)=\mu(X)$, for all $X$ in $V$ and $\mu^{\prime}(\hat{A})=\{\hat{B}: B$ is in $\mu(A)\}$ for all $A$ in $V-\Sigma$. Let

$$
\begin{aligned}
P_{F}^{\prime} & =\left\{A \rightarrow W, \hat{A} \rightarrow W: A \rightarrow W \text { is in } P^{\prime}, W \text { in } \Sigma^{*}\right\} \\
& \cup\left\{A \rightarrow \hat{B} C, \hat{A} \rightarrow \hat{B} C: A \rightarrow B C \text { is in } P^{\prime}, B, C \text { in } V^{\prime}-\Sigma^{\prime}\right\}
\end{aligned}
$$

and $F^{\prime}=\left(V_{F}^{\prime}, \Sigma^{\prime}, P_{F}^{\prime}, S^{\prime}\right)$ where $V_{F}^{\prime}=V^{\prime} \cup\left\{\hat{A}: A\right.$ is in $\left.V^{\prime}-\Sigma^{\prime}\right\}$. Then $F^{\prime} \underset{x}{ } F\left(\mu^{\prime}\right)$ and $L\left(F^{\prime}, \Rightarrow\right)=L\left(G^{\prime}, \Rightarrow\right)$. Hence $\mathcal{L}_{x}(G, \Rightarrow) \subseteq \mathcal{K}_{x}(F, \Rightarrow)$ completing the proof.

\section{II.5.3 Pushdown Acceptor Forms}

We develop the notion of a pda form under both g- and $\mathrm{s}-$ interpretations.

Recall that a pushdown acceptor (pda) is a sextuple $M=\left(Q, \Sigma, \Gamma, H, Z_{0}, q_{0}\right)$ where $Q$ is a finite set of states, $\Sigma$ is the input alphabet, $\Gamma$ is the pushdown alphabet, $H$ is a finite subset of $Q \times \cdots(\Sigma \cup\{\lambda\}) \times \Gamma \times \stackrel{\star}{\Gamma} \times Q$ of moves, $Z_{0}$ is the initial pushdown symbol and $q_{0}$ is the initial state. Often a set of final states is also given, but for our purposes this is an unncessary addition as will be seen. Also $H$ the move set is usually given as a transition function $\delta: Q \times(\Sigma \cup\{\lambda\}) \times \Gamma \rightarrow 2^{\Gamma^{*} \times Q}$, however this is clearly an equivalent formulation.

Given a $p d a M$ define the relation

$$
\begin{aligned}
& (p, x, \gamma) \longmapsto\left(q, x^{\prime}, \gamma^{\prime}\right) \text { if } x=z x^{\prime}, \gamma=z \delta \text { and } \\
& \gamma^{\prime}=\delta^{\prime} \delta \text { where }\left(p, z, z, \delta^{\prime}, q\right) \text { is in } H .
\end{aligned}
$$

observe that the stack $\gamma$ is being read from left to right. Let be the reflexive transitive closure of trand let $\operatorname{Null}(M)=\left\{x: x\right.$ in $\Sigma^{*},\left(q_{0}, x, z_{0}\right) \vdash^{\star}(q, \lambda, \lambda)$ for some $q$ in $\left.Q\right\}$. It is well known that the family of all Null(M) is identical to $\mathcal{L}(C F)$.

A pda form is a pda, in the same way that a grammar form is a grammar. 


\section{Definition}

Let $M_{1}$ and $M_{2}$ be pda forms, where $M_{i}=\left(Q_{i}, \Sigma_{i}, \Gamma_{i}, H_{i}, Z_{i}, 0, q_{i, 0}\right)$ $i=1,2$. We say $\underline{M}_{2}$ is an s-interpretation of $M_{1}$ modulo $\mu$, where $\mu$ is a dfl-substitution from $Q_{1} \cup \Sigma_{\Sigma_{1}} \cup \Gamma_{1}$ into $Q_{2} \cup \Sigma_{2} \cup \Gamma_{2}$, if

(i) $\mu\left(Q_{1}\right) \subseteq Q_{2}$,

(ii) $\mu\left(\Sigma_{1}\right) \subseteq \Sigma_{2}$,

(iii) $\mu\left(r_{1}\right) \subseteq \Gamma_{2}$,

(iv) $H_{2} \subseteq \mu\left(H_{1}\right)$, where $\mu\left(H_{1}\right)=\underbrace{}_{h \text { in } H_{1}} \mu(h)$ and $\mu((p, x, Z, \gamma, q))=\mu(p) \times \mu(x) \times \mu(Z) \times \mu(\gamma) \times \mu(q)$,

(v) $z_{2,0}$ is in $\mu\left(z_{1,0}\right)$, and

(vi) $q_{2,0}$ is in $\mu\left(q_{1,0}\right)$.

We denote this by $M_{2} \underset{s}{\vec{S}} M_{1}(\mu)$, or simply $M_{2} \underset{s}{\Delta} M_{1}$ when $\mu$ is understood. The family of pda forms defined by a pda form $M$ is denoted $m_{s}(M)$ and defined by:

$m_{s}(M)=\left\{M^{\prime} \vec{s} M(\mu):\right.$ for some substitution $\mu$ and $\left.p d a M^{\prime}\right\}$.

Similary by $\mathcal{L}_{s}(M)$ we denote the family of languages given by

$M$, defined by:

$\mathcal{L}_{S}(M)=\left\{N u 11\left(M^{\prime}\right): M^{:}\right.$is in $\left.m_{s}(M)\right\}$.

These are known as the s-pda family and the s-pda language family of $M$, respectively.

Before proving our major result, namely that for each grammar form $G$ there is a pda form $M$ such that $\mathcal{X}_{S}(G, \Rightarrow)=\mathcal{L}_{S}$ (M) and vice versa, we prove a normal form result for $s-p d a$ families. For each 1 -state pda form $M$ and each $s$-interpretation $M^{\prime}$ of $M$ there is an $s$-interpretation $M^{\prime \prime}$ of $M$ such that $M^{\prime \prime}$ is a 1 -state pda and $\operatorname{Nu} 11\left(M^{\prime \prime}\right)=\operatorname{Nul1}\left(M^{\prime}\right)$.

\section{Notation}

For each pda $M=\left(Q, \Sigma, \Gamma, H, Z_{0}, q_{0}\right)$ and for $Z_{j}, \ldots, Z_{k}$ in $\Gamma, k \geq 1$, and $q, q^{\prime}$ in $Q$ let

$$
\begin{gathered}
\left\langle q, z_{1} \ldots z_{k}, q^{\prime}\right\rangle=\left\{\left(q, z_{1}, q_{1}\right)\left(q_{1}, z_{2}, q_{2}\right) \ldots\left(q_{k-1}, z_{k}, q^{\prime}\right):\right. \\
\left.q_{1}, \ldots, q_{k-1} \text { in } Q\right\} .
\end{gathered}
$$

Let $\bar{M}=\left(Q, \Sigma,(Q \times \Gamma \times Q) \cup\left\{Z_{0}\right\}, \bar{H}, Z_{0}, q_{0}\right)$ be the pda in which $\bar{H}$ is defined by:

(i) if $h=\left(q_{0}, x, z_{0}, \lambda, q\right)$ is in $H$ then $h$ is in $\bar{H}$.

(ii) if $\left(q, x, z, \lambda, q^{\prime}\right)$ is in $H$ then $\left(q, x,\left(q, z, q^{\prime}\right), \lambda, q^{\prime}\right)$ is in $\bar{H}$.

(iii) if $\left(q_{0}, x, Z_{0}, \gamma, q\right)$ is in $H, \gamma \neq \lambda$, then $\left(q_{0}, x, Z_{0}, \gamma, q\right)$ is in

A for a $11 \gamma^{\prime}$ in $\left\langle q, \gamma, q^{\prime}\right\rangle$ and a $11 q^{\prime}$ in $Q$.

(iv) if $\left(q, x, Z, \gamma, q^{\prime}\right)$ is in $H, \gamma \neq \lambda$, then $\left(q, x,\left(q, Z, q^{\prime \prime}\right), \gamma^{\prime}, q^{\prime}\right)$ is in $\bar{H}$ for a $11 \gamma^{\prime}$ in $\left\langle q^{\prime}, \gamma, q^{\prime \prime}\right\rangle$ and $a 11 q^{\prime \prime}$ in $Q$. 
Let $\overline{\bar{M}}=\left(\left\{q_{0}\right\}, \Sigma,(Q \times \Gamma \times Q) \cup\left\{Z_{0}\right\}, \overline{\bar{H}}, Z_{0}, q_{0}\right)$ be the pda in which $\left(q_{0}, x, Z, \gamma, q_{0}\right)$ is in $\overline{\bar{H}}$ if $\left(q, x, Z, Y, q^{\prime}\right)$ is in $\bar{H}$ for some $q, q^{\prime}$ in $Q$.

observe that, by construction, in $\bar{M}$ a move of the type $\left(q, x,\left(p, z, p^{\prime}\right) \gamma\right) \vdash\left(q^{\prime}, x^{\prime}, \gamma^{\prime}\right)$ may only take place when $p=q$ and, secondly, $\gamma=\gamma^{\prime}$, that is an erasing move takes place, only when $p^{\prime}=q^{\prime}$.

We relate $M, \bar{M}$ and $\overline{\bar{M}}$ in the following lemma.

\section{Lemma 5.10}

Let $M, \bar{M}$ and $\overline{\bar{M}}$ be defined as above. Then $N u 11(\bar{M})=N u 11(\overline{\bar{M}})$ $=\operatorname{Nul1}(M)$.

Proof: We first sketch a proof that Null(M) $=$ Null(M). Consider an accepting move sequence in $M$ :

$$
\left(q_{0}, x_{1} \ldots x_{m}, z_{0}\right) \vdash\left(q_{1}, x_{2} \ldots x_{m}, \gamma_{1}\right) \vdash \ldots \vdash\left(q_{m-1}, x_{m}, \gamma_{m-1}\right) \vdash\left(q_{m}, \lambda, \lambda\right)
$$
where $x_{i}$ is in $\left.\sum u\{\lambda\},\right] \leq i \leq m$.

Now $\gamma_{m-1}$ is in $\Gamma$ and since it is erased, then either $\gamma_{m-1}=z_{0}$ in which case $\left(q_{m-1}, x_{m}, \gamma_{m-1}\right) \vdash\left(q_{m}, \lambda, \lambda\right)$ is in $\bar{M}$ also or $\gamma_{m-1} \neq Z_{0}$ in which case $\left(q_{m-1}, x_{m},\left(q_{m-1}, \gamma_{m-1}, q_{m}\right)\right) \leftarrow\left(q_{m}, \lambda, \lambda\right)$ is in $\bar{M}$ by the previous observations. Moreover, in both cases, the corresponding move in $\bar{M}$ is uniquely determined. By induction on $i$ there is a unique $\delta_{j}$ in $\left\langle q_{i}, \gamma_{i}, q_{m}\right\rangle$ for each $i, l \leq i \leq m-l$. Hence in $\bar{M}$ there is a uniquely determined accepting move sequence:

$$
\left(q_{0}, x_{1} \ldots x_{m}, z_{0}\right) \vdash\left(q_{1}, x_{2} \ldots x_{m}, \delta_{1}\right) \vdash \ldots \ldots\left(q_{m-1}, x_{m}, \delta_{m-1}\right) \vdash\left(q_{m}, \lambda, \lambda\right)
$$
with $\delta_{i}$ in $\left\langle q_{i}, \gamma_{i}, q_{m}\right\rangle$. Thus Nu11 (M) $\subseteq N u 11(\bar{M})$.

Conversely, given an accepting move sequence:

$$
\left(q_{0}, x_{1} \ldots x_{m}, z_{0}\right) \vdash \ldots \leftarrow\left(q_{m-1}, x_{m}, \delta_{m-1}\right) \vdash\left(q_{m}, \lambda, \lambda\right)
$$

in $\bar{M}$, then $\delta_{j}$ is in $\left\langle q_{i}, \gamma_{j}, q_{m}>\right.$ for some unique $\gamma_{i}$ and therefore:

$$
\left(q_{0}, x_{1} \ldots x_{m}, z_{0}\right) \longmapsto \ldots \vdash\left(q_{m-1}, x_{m}, \gamma_{m-1}\right) \longmapsto\left(q_{m}, \lambda, \lambda\right)
$$

is an accepting move sequence in $M$. We have now shown that

$\operatorname{Nul1}(\bar{M}) \subseteq N u 11(M)$ and hence Nu11( $\bar{M})=N u 11(M)$.

We close by demonstrating that $N$ ull $(\overline{\bar{M}})=\operatorname{Null}(\bar{M})$.. By definition of $\overline{\bar{M}}$, for each accepting move sequence:

$$
\left(q_{0}, x_{1} \ldots x_{m}, i_{0}\right) \vdash\left(q_{1}, x_{2} \ldots x_{m}, \delta_{1}\right) \vdash \ldots \vdash\left(q_{m}, \lambda, \lambda\right)
$$

in $M$ there is in accepting move sequence:

$$
\left(q_{0}=x_{1} \ldots x_{m}, z_{0}\right) \vdash\left(q_{0}, x_{2} \ldots x_{m}, \delta_{1}\right) \vdash \ldots \vdash\left(q_{0}, \lambda, \lambda\right)
$$

in $\overline{\bar{M}}$. Conversely, given an accepting move sequence in $\overline{\bar{M}}$ the $\delta_{i}, 1 \leq i \leq m-1$ uniquely determine the corresponding $q_{i}, 1 \leq i \leq m$. Hence there is a uniquely determined accepting move sequence in $\bar{M}$. This gives the result. 
Lemma 5.11

Let $M, \bar{M}$ and $\overline{\bar{M}}$ be given as above. Then $\bar{M} \underset{S}{\Delta} M, \bar{M} \underset{S}{\Delta} \overline{\bar{M}}$ and moreover if $M$ is a 1 -state $p d a$ and $M^{\prime} \rightarrow{ }_{s}(\mu)$ for some $p d a M^{\prime}$, then $\overline{\bar{M}}^{\prime} \rightarrow M$.

Proof: (i) $\bar{M} \underset{s}{4} M$. Define a dfl-substitution $\mu$ by:

$$
\begin{aligned}
& \mu(q)=q, \text { for a } 11 \text { q in } Q, \\
& \mu(a)=a, \text { for a } 11 \text { a in } \Sigma, \\
& \mu\left(Z_{0}\right)=\left\{Z_{0}\right\} \cup\left(Q \times\left\{Z_{0}\right\} \times Q\right) \text { and } \\
& \mu(Z)=Q \times\{Z\} \times Q \text {, for all } Z \text { in } \Gamma-\left\{Z_{0}\right\} .
\end{aligned}
$$

Clearly $\bar{H} \subseteq \mu(H)$, hence $\bar{M} \underset{S}{\triangle} M(\mu)$.

(ii) $\bar{M} \underset{s}{\Delta} \overline{\bar{M}}$. Define a dfl-substitution $\mu$ by:

$\mu\left(q_{0}\right)=Q$,

$\mu(X)=X$ for a $11 X$ in $\Sigma \cup \Gamma$.

Then $\bar{H} \subseteq \mu(\overline{\bar{H}})$ by construction, hence $\bar{M} \underset{s}{\Delta} \overline{\bar{M}}(\mu)$.

(iii) If $\# Q>1$ then $\overline{\bar{M}}$, and hence $\overline{\bar{M}}$ ', cannot be an s-interpretation of

M. When $M$ is a 1 -state pda define a dfl-substitution $\overline{\bar{\mu}}$ by:

$$
\begin{aligned}
& \overline{\bar{\mu}}\left(q_{0}\right)=q_{0}, \\
& \overline{\bar{\mu}}(a)=\mu(a) \text {, for a } 11 \text { a in } \Sigma, \\
& \overline{\bar{\mu}}\left(Z_{0}\right)=\left\{Z_{0}^{\prime}\right\} \times\left(Q^{\prime} \times \mu\left(Z_{0}\right) \times Q^{\prime}\right) \text { and } \\
& \left.\overline{\bar{\mu}}(Z)=Q^{\prime} \times \mu(Z) \times Q^{\prime} \text {, for a } 1\right] Z \text { in } \Gamma-\left\{Z_{0}\right\} \text {. }
\end{aligned}
$$

Immediately $\overline{\bar{H}}^{\prime} \subseteq \overline{\bar{\mu}}(H)$, hence $\overline{\bar{M}},{ }_{s} M(\overline{\bar{\mu}})$.

Our promised "normal form" result now follows:

\section{Theorem 5.12}

Let $M$ be a 1 -state pda form. Then for all $M^{\prime} \rightarrow M$ there exists an $\overline{\bar{M}} \cdot \vec{s} M$ with $N u l l\left(\overline{\bar{M}}^{\prime}\right)=N u l l\left(M^{\prime}\right)$ and $\overline{\bar{M}}^{\prime}$ a 1 -state pda.

This result for pda families corresponds to the well known result that the family of 1 -state pda's generates the family of all pda languages, that is all context-free languages. We now proceed to strengthen this correspondence to show that every pda language family is a grammatical family and vice versa.

Consider the following well known 1-state pda $M_{G}$ corresponding to a given grammar $G$.

\section{Definition}

Let $G=(V, \Sigma, P, S)$ be a grammar. Let $M_{G}$, the corresponding 1-state $p d a$ of $G$, be the $p d a\left(\left\{q_{0}\right\}, \Sigma, V, H, S, q_{0}\right)$ where 
$H=\left\{\left(q_{0}, a, a, \lambda, q_{0}\right): a\right.$ in $\left.\Sigma\right\} \cup\left\{\left(q_{0}, \lambda, A, \alpha, q_{0}\right): A \rightarrow \alpha\right.$ is in $\left.P\right\}$.

It is well known that $\operatorname{Null}\left(M_{G}\right)=L(G, \Rightarrow)$, for all context-free grammars $G$.

\section{Theorem 5.13}

For every grammar $G, \mathcal{Z}_{S}(G, \Rightarrow)=\mathcal{L}_{S}\left(M_{G}\right)$.

Proof: Letting $G^{\prime} \rightarrow G(\mu)$, where $G^{\prime}=\left(V^{\prime}, \Sigma^{\prime}, P^{\prime}, S^{\prime}\right)$ the pda corresponding to $G^{\prime}$ is given by:

$$
\begin{gathered}
M^{\prime}=\left(\left\{q_{0}\right\}, \Sigma^{\prime}, V^{\prime}, H^{\prime}, S^{\prime}, q_{0}\right) \text {, where } H^{\prime}=\left\{\left(q_{0}, a^{\prime}, a^{\prime}, \lambda, q_{0}\right): \text { a in } \Sigma^{\prime}\right\} \\
\cup\left\{\left(q_{0}, \lambda, A^{\prime}, \alpha^{\prime}, q_{0}\right): A^{\prime} \rightarrow \alpha^{\prime} \text { is in } P\right\} .
\end{gathered}
$$

Let $\bar{\mu}$ be a dfl-substitution defined by:

$\vec{\mu}\left(q_{0}\right)=q_{0}$ and

$\bar{\mu}(X)=\mu(X)$, for $a 11 X$ in $V$.

Clearly $M^{\prime} \Delta M_{G}(\bar{\mu})$ and $N u T l\left(M^{\prime}\right)=L\left(G^{\prime}, \Rightarrow\right)$ as pointed out above.

Therefore $\mathcal{Z}_{\mathrm{s}}(G, \Rightarrow) \subseteq \mathcal{L}_{\mathrm{s}}\left(M_{\mathrm{G}}\right)$.

Consider the reverse inclusion. Since $M_{G}$ is a 1 -state pda we need only consider 1 -state s-interpretations of $M_{G}$ by Theorem 5.12. Let $M^{\prime}=\left(\left\{q_{0}\right\}, \Sigma^{\prime}, \Gamma^{\prime}, H^{\prime}, Z_{0}^{\prime}, q_{0}\right)$ and $M^{\prime} \underset{S}{A} M(\mu)$. Since $H^{\prime} \subseteq \mu(H)$ each move in $H^{\prime}$ is either of the form:

(i) $\left(q_{0}, a^{\prime}, a^{\prime \prime}, \lambda, q_{0}\right)$, where $a^{\prime}$ and $a^{\prime \prime}$ are in $\mu(a)$, for some $a$ in $\Sigma$, or of the form

(ii) $\left(q_{0}, \lambda, A^{\prime}, \alpha^{\prime}, q_{0}\right)$, where $A^{\prime} \rightarrow \alpha^{\prime}$ is in $\mu(A \rightarrow \alpha)$ for some $A \rightarrow \alpha$ in $P$. Type ( $i$ ) moves mean that a" on the pushdown can match a symbol a' in the input. Define $\tau\left(a^{\prime \prime}\right)=\left\{a^{\prime}:\left(q_{0}, a^{\prime}, a^{\prime \prime}, \lambda, q_{0}\right)\right.$ is in $\left.H^{\prime}\right\}$, for all $a^{\prime \prime}$ in $\Sigma^{\prime}$ and $\tau(X)=X$, for all $x$ in $\left\{q_{0}\right\} \cup r$. Note that $\tau$ is not necessarily a dfl-substitution but $\tau(\mu(a)) \subseteq \mu(a)$ for all a in $\Sigma$, hence $\tau \mu$ is a dff-substitution. Let $G^{\prime}=\left(\Gamma^{\prime} \cup \Sigma^{\prime}, \Sigma^{\prime}, P^{\prime}, Z_{0}^{\prime}\right)$ where $P^{\prime}=\left\{A^{\prime} \rightarrow \alpha^{\prime \prime}: \alpha^{\prime \prime}\right.$ is in $\tau\left(\alpha^{\prime}\right),\left(q_{0}, \lambda, A^{\prime}, \alpha^{\prime}, q_{0}\right)$ is in $\left.H^{\prime}\right\}$. Then $G^{\prime} \vec{S} G(\tau \mu)$. The proof that $\operatorname{Null}^{\prime}\left(M^{\prime}\right)=L\left(G^{\prime}, \Rightarrow\right)$ is straightforward and is omitted. Thus $\mathcal{Z}_{\mathrm{S}}\left(M_{\mathrm{G}}\right) \subseteq \mathcal{Z}_{\mathrm{S}}(G, \Rightarrow)$ completing the proof.

We now turn to the converse result.

\section{Definition}

Let $M=\left(Q, \Sigma, \Gamma, H, Z_{0}, q_{0}\right)$ be a pda and $S$ a new symbol. Define the corresponding grammar $G_{M}=(\{S\} \cup \Sigma \cup(Q \times \Gamma \times Q), \Sigma, P, S)$ by specifying $P$ as follows:

(i) if $\left(q_{0}, x, Z_{0}, \lambda, q^{\prime}\right)$ is in $H$, then $S \rightarrow x$ is in $P$.

(ii) if $\left(q, x, z, \lambda, q^{\prime}\right)$ is in $H$, then $\left(q, z, q^{\prime}\right) \rightarrow x$ is in $p$. 
(iii) if $\left(q_{0}, x, Z_{0}, Z_{1}, \ldots z_{r}, q\right)$ is in $H$, then

$s+x\left(q, z_{1}, q_{1}\right)\left(q_{1}, z_{2}, q_{2}\right) \ldots\left(q_{r-1}, z_{r}, q_{r}\right)$ is in $p$, for all

$q_{1}, \ldots, q_{r}$ in $Q$.

(iv) if $\left(q, x, Z, Z_{1} \ldots z_{r}, q^{\prime}\right)$ is in $H$, then

$\left(q, z, q^{\prime \prime}\right) \rightarrow x\left(q^{\prime}, z_{\eta}, q_{1}\right)\left(q_{1}, z_{2}, q_{2}\right) \ldots\left(q_{r-1}, z_{r}, q^{\prime \prime}\right)$

is in $P$ for a $11 q^{\prime \prime}, q_{1}, \ldots, q_{r}$ in $Q$.

It is well known that $L\left(G_{M} \Rightarrow\right)=N u 11(M)$.

Theorem 5.14

For every pda $M, \mathcal{L}_{S}\left(G_{M} \Rightarrow\right)=\mathcal{L}_{S}(M)$.

Proof: Let $M^{\prime}=\left(Q^{\prime}, \Sigma^{\prime}, \Gamma^{\prime}, H^{\prime}, Z_{0}^{\prime}, q_{0}^{\prime}\right)$ and $M^{\prime} \Delta M(\mu)$. Then

$G^{\prime}=\left(\{S\} \cup \Sigma^{\prime} \cup\left(Q^{\prime} \times \Gamma^{\prime} \times Q^{\prime}\right), \Sigma^{\prime}, P^{\prime}, S\right)$ can be constructed in the manner specified above and it is clear that $G: \bigotimes_{S} G_{M}(\bar{\mu})$, where $\bar{\mu}$ is defined by:

$\bar{\mu}(S)=S, \vec{\mu}(a)=\mu(a)$, for a 11 a in $\Sigma$, and

$\bar{\mu}((p, Z, q))=\left\{\left(p^{\prime}, Z^{\prime}, q^{\prime}\right): p^{\prime}\right.$ in $\mu(p), Z^{\prime}$ in $\mu(Z)$ and $q^{\prime}$ in $\mu(q)\}$, for all $(p, Z, q)$ in $Q \times \Gamma \times Q$.

Hence $\mathcal{L}_{\mathrm{S}}(M) \subseteq \mathcal{L}_{\mathrm{S}}\left(G_{M}, \Rightarrow\right)$. Consider the converse result. Let $G^{\prime} \triangle G_{M}(\mu)$ where $G^{\prime}=\left(V^{\prime}, \Sigma^{\prime}, P^{\prime}, S^{\prime}\right)$. Also let

$\tilde{M}=\left(Q, \Sigma,(Q \times \Gamma \times Q) \cup\left\{Z_{0}\right\}, \vec{H}, Z_{0}, q_{0}\right)$ be the specific s-interpretation pda of $M$ introduced earlier. Since $\bar{M} \vec{s} M$ it suffices to demonstrate a pda $\bar{M}^{\prime} \triangleleft_{s} \bar{M}(\bar{\mu})$ with $N u l l\left(\bar{M}^{\prime}\right)=L\left(G^{\prime}, \Rightarrow\right)$. Define $\bar{\mu}$ as follows:

$\bar{\mu}(X)=X$, for al1 $X$ in $Q \cup \Sigma$,

$\bar{\mu}\left(z_{0}\right)=\mu(s)$ and $\bar{\mu}((p, Z, q))=\mu((p, Z, q))$ for a 1$](p, z, q)$ in $Q \times \Gamma \times Q$. clearly $\bar{\mu}$ is a dfl-substitution. Let $\bar{M}^{\prime}=\left(Q, \Sigma^{\prime}, V^{\prime}, H^{\prime}, S^{\prime}, q_{0}\right)$, where $H^{\prime}$ consists of the following moves:

(i) if $S^{\prime} \rightarrow x^{\prime}$ is in $P^{\prime}, S^{\prime} \rightarrow x^{\prime}$ is in $\mu(S \rightarrow x)$ and $S \rightarrow x$ is type (i) (by which we mean $S \rightarrow x$ is obtained from the move $\left(q_{0}, x, Z_{0}, \lambda, q^{\prime}\right)$ in $H$, fulfilling condition (i) in the definition of $\left.G_{M}\right)$, then let $\left(q_{0}, x^{\prime}, S^{\prime}, \lambda, q^{\prime}\right)$ be in $H^{\prime}$.

(ii) If $A \rightarrow x^{\prime}$ is in $P^{\prime}, A \rightarrow x^{\prime}$ is the image of a type (ii) production coming from $\left(q, x, Z, \lambda, q^{\prime}\right)$ then let $\left(q, x^{\prime}, A, \lambda, q^{\prime}\right)$ be in $H^{\prime}$.

(iii) if $S^{\prime} \rightarrow x^{\prime} A_{1} \ldots A_{r}$ is in $P^{\prime}$, the image of a type (iii) production coming from $\left(q_{0}, x, z_{0}, z_{\eta} \ldots z_{r}, q\right)$ then let $\left(q_{0}, x^{\prime}, S^{\prime}, A_{1} \ldots A_{r}, q\right)$ be in $H^{\prime}$.

(iv) if $A \rightarrow x^{\prime} A_{1} \ldots A_{r}$ is in $P^{\prime}$, the image of a type (iv) production coming from $\left(q, x, Z, Z_{1} \ldots Z_{r}, q^{\prime}\right)$ in $H$, let $\left(q, x^{\prime}, A, A_{1} \ldots A_{r}, q^{\prime}\right)$ be in $H^{\prime}$. 
Clearly $\bar{M} \cdot \underset{S}{\Delta}(\bar{\mu})$. Finally, note that in $G_{M}$, $S$ only appears on the lefthand side of productions, all other nonterminals are in $Q \times \Gamma \times Q$.

It follows that in a leftmost derivation in $G^{\prime}, S^{\prime} L^{*} \alpha$ in $G^{\prime}$ implies that $\alpha=u \beta$ for some $u$ in $\Sigma^{\prime *}$ and $\beta$ in $\mu(Q \times \Gamma \times Q)^{*}$. By induction on the length of the derivation, $n \geq 1$, it $c$ an be shown that:

$S^{\prime} \Rightarrow n \quad u \beta$ in $G^{\prime}$ iff there is an $n$-step move sequence from $\left(q_{0}, u, S^{\prime}\right)$ to $(q, \lambda, \beta)$ for some $q$ in $Q$.

Thus Null $\left(\bar{M}^{\prime}\right)=L\left(G^{\prime}, \Rightarrow\right)$, completing the proof.

We can summarize the results so far in the following theorem.

Theorem 5.15 For each family of languages $\mathcal{L}, \mathcal{Z}$ is an s-pda language famity
iff $\mathcal{L}$ is an s-grammatical family.

We now turn to g-interpretations of pda forms.

First observe that the definition of g-interpretation for pda forms, which is analogous to that for grammar forms, will normally produce acceptors as interpretations which are not pdas. This is because the image of an input symbol may be a word. We therefore allow a more general definition of a pda in which $(p, x, z, \gamma, q)$ is an allowable move even when $|x|>1$. We call this a generalized pda or simply gpda. The definition of a move sequence is appropriately modified, in which case it is clear that if $M_{1}$ is a gpda then there exists a pda $M_{2}$ such that $\operatorname{Nu} 11\left(M_{1}\right)=\operatorname{Null}\left(M_{2}\right)$. In other words no generative power is added.

We say $M_{1}$ is a g-interpretation of $M_{2}$ modulo $\mu$, denoted $M_{2} \underset{g}{\Delta} M_{1}(\mu)$, if $M_{i}=\left(Q_{i}, \Sigma_{i}, \Gamma_{i}, H_{i}, z_{i, 0}, q_{i, 0}\right), i=1,2$ and $\mu$ is $a$ finite substitution from $Q_{1} \cup \Sigma_{1} \cup \Gamma_{1}$ to $Q_{2} \cup \Sigma_{2}^{*} \cup \Gamma_{2}$, which fulfills conditions (i), ( $i j i),(i v),(v)$ and ( $i$ ) in the definition of s-interpretations and (ii) is replaced by:

(ii') $\mu\left(\Sigma_{\eta}\right) \subseteq \Sigma_{2}^{*}$.

We obtain $m_{g}(M)$ and $\mathcal{L}_{g}(M)$ analogously to $m_{s}(M)$ and $\mathcal{L}_{s}(M)$. The following results are straightforward and are left to the reader.

Proposition 5.16

For each gpda form $M_{1}$ there is a strong $g$-form equivalent pda form $M_{2}$. 
Proposition 5.17

For each pda form $M$, and hence each gpda form, $\mathcal{L}_{g}(M)=\mathcal{H}\left(\mathcal{L}_{S}(M)\right)$.

our main result now follows straightforwardly.

\section{Theorem 5.18}

For each language family $\mathcal{L}$ the following are equivalent statements:

(i) $\mathcal{L}$ is a g-grammatical family.

(ii) $\mathcal{L}$ is a g-pda language family.

(iii) $\mathcal{L}$ is a g-gpda language family.

Proof: (ii) $\equiv$ (iii) follows from Proposition 5.16. We show that $(i) \equiv(i i)$. Now by Theorem 5.15 a language family $\mathcal{L}$ is 5 -grammatical iff it is an s-pda language family.

Now $\mathcal{Z}=\mathcal{L}_{\mathrm{g}}(G, \Rightarrow$, for some grammar form $G$

iff $=\mathcal{H}^{g}\left(\mathcal{L}_{\mathrm{S}}\left(G_{s} \Rightarrow\right)\right)$

iff $=\mathcal{H}\left(\mathcal{Z}_{\mathrm{S}}(M)\right)$, for some $p d a$ form $M$ by Theorem 5.15

iff $=\mathcal{L}_{g}(M)$. Hence the result.

We have demonstrated in this section that the analogue for pda's of s-and g-interpretations for grammar forms yields exactly the same families of languages. This means that pda forms correspond to grammar forms in the same way that pdas corresponds to grammars. Thus for each s-or g-grammatical family we immediately have available a parsing algorithm for the whole family. In the same way that deterministic pda (dpda) have been studied as models of realistic parsing techniques (1inear in the length of the input word) for grammars, so the study of dpda within an s-or g-pda family may now be investigated with the same end in view. This is an area of investigation for which little is known at the time of writing.

In closing we mention one further result which the interested reader may prove for himself.

\section{Proposition 5.19}

$$
\begin{aligned}
& \text { For every gpda } M_{1} \text { there is a pda } M_{2} \text { such that } \\
& \mathcal{Z}_{s}\left(M_{1}\right)=\mathcal{Z}_{s}\left(M_{2}\right) \text {. }
\end{aligned}
$$

Thus even under s-interpretations gpda's are no more powerful than pda's. 


\section{6 Dense Collections of Grammatical Families}

The framework of grammar forms provides us not only with the twin notions of a family of grammars and a grammatical family with respect to a given grammar but also it provides us with collections of these in a natural way. In Section II.3 the collection of the families of grammars defined by context-free grammar forms was studied. In the present section we study the corresponding collection of contextfree grammatical families under s-interpretations. We show, in Section 6.2, that the collection of s-grammatical families is dense in the sense that given two families $\mathcal{L}_{1}$ and $\mathcal{Z}_{2}$ such that $\mathcal{Z}_{1} \subsetneq \mathcal{Z}_{2}$ and $\mathcal{Z}_{1}$ contains all finite sets then there always exists a family $\mathcal{Z}_{3}$ properly in between, that is

$$
\mathcal{Z}_{1} \subsetneq \mathcal{Z}_{3} \subsetneq \mathcal{Z}_{2} \text {. }
$$

Second, in Section 6.3 we abstract the notions that enable such a density result to be proved using the techniques of the first section. This leads in a natural way to collections of language families fulfilling some basic properties, which we call MSW spaces. We demonstrate that such collections are not a rare occurrence, in that given an arbitrary collection $M$ we can always obtain an MSW space by closing $M$ under some operators in a fixed finite sequence, which is exactly the closure of $m$ under these particular operators. Finaliy, in Section 6.4, we demonstrate a density result for two-symbol-sgrammatical families, which leads to the decidability of form equivalence for sub-linear two-symbol-s-grammatical families. These two density results are of interest since they are established in two very different ways and also because such results have not been forthcoming in the past when generative devices have been studied.

\section{II.6.1 Preliminary Notions}

In order to prove the results on density it is convenient to introduce some notation and also a number of language and language family operations.

We say that a collection $M$ of language families is dense if for any two language families $\mathcal{L}_{1}$ and $\mathcal{L}_{2}$ in $M_{\text {with }} \mathcal{L}_{1} \underset{\mathcal{Z}}{\mathcal{Z}_{2}}$ there exists a language family $\mathcal{L}$ in $\mathcal{M}$ strictly in between, that is

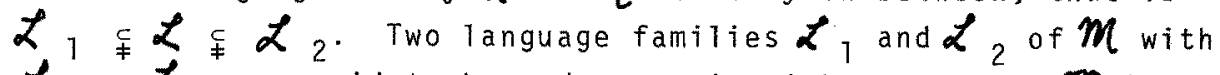
$\mathcal{Z} \mathcal{Z}_{2}$ are said to be a dense pair with respect to $m$ if $m\left(\mathcal{Z}_{1}, \mathcal{L}_{2}\right)=\left\{\mathcal{L}\right.$ in $\left.m: \mathcal{L}_{1} \leq \mathcal{Z} \leq \mathcal{L}_{3}\right\}$ is dense, we normal1y write $\left(\mathcal{L}_{1}, \mathcal{L}_{2}\right)$ is a dense pair. If $\mathcal{L}_{1 \neq} \mathcal{L}_{2}$ and there is no $\mathcal{Z}_{3}$ in $m$ such that $\mathcal{L}_{1}$ 娄 $\mathcal{L}_{2}$, then $\mathcal{L}_{2}$ is a successor of $\mathcal{L}_{1}$. We say in $\mathcal{L}_{1}$ in $M$ is density forcing with respect to $m$ if 
$m\left[\mathcal{L}_{1}\right]=\left\{\mathcal{L}\right.$ in $\left.m: \mathcal{L}_{1} \subseteq \mathcal{L}\right\}$ is dense.

In the next section we will prove that $\mathcal{L}(R E G)$ is density forcing with respect to the collection of all s-grammatical families and also that $(\mathcal{Z}(R E G), \mathcal{L}(C F))$ is a dense pair.

We now introduce some necessary language and language family operations.

Let $L_{1}$ and $L_{2}$ be two languages over disjoint alphabets. Then the superdisjoint union of $L_{1}$ and $L_{2}$, denoted by $L_{1} \boxminus L_{2}$, is simply the union of $L_{1}$ and $L_{2}$. Note that the superdisjoint union is only defined if the two languages are over disjoint alphabets. Similarly we define a kind of inverse of this operation. Let $L$ be a language over some alphabet $\Sigma$. Then a language $L_{1}$ is obtained by breaking $L$ with respect to an alphabet $\Sigma_{1} \subseteq \Sigma$ if $L_{1}=L \cap \Sigma_{j}^{*}$ and $L-L_{1} \subseteq\left(\Sigma-\Sigma_{1}\right) *$, that is $L-L_{1}$ does not contain any word containing a symbol of $\Sigma_{1}$. We say that $L$ is coherent if it cannot be broken in a non-trivial manner. So if $L_{1}$ is obtained by breaking $L$ then either $L_{1}=L$ or $L_{1}=\emptyset$.

The operation of superdisjoint union can be extended to language families as follows.

The superdisjoint wedge of two language families $\mathcal{L}_{1}$ and $\mathcal{L}_{2}$, denoted by $\mathcal{Z}_{1} \forall \mathcal{L}_{2}$, is defined by: $\mathcal{L}_{1} \dot{V} \mathcal{Z}_{2}=\left\{L_{1} \boldsymbol{U} L_{2}: L_{1}\right.$ in $\mathcal{L}_{1}$ and $\mathrm{L}_{2}$ in $\mathcal{Z}_{2}$.

Another useful operation for both languages and language families is that of removing all words of a given length from a language or from a 11 languages in a family. Let $L$ be a language and $\mathcal{L}$ a language family. Then for $i \geq 1$ we denote by $L(i)$ the language defined by $\{x$ is in $L:|x| \neq i\}$. Similarly by $\mathcal{L}(i)$, we denote the language family $\{L(i)=L$ is in $\mathcal{L}\}$. We call $L(i)$ an extraction of $L$ and $\mathcal{L}(i)$ an extraction of $\mathcal{L}$.

A language family $\mathcal{L}$ is closed under covering if for every infinite language $L$, the fact that $L(i)$ is in $\mathcal{L}$ for infinitely many $i$ implies that $L$ is in $\mathcal{L}$.

We will provide a grammatical characterization of superdisjoint wedge by way of the following operation on grammars.

Let $G_{i}=\left(V_{i}, \Sigma_{i}, P_{i}, S_{i}\right)$ be two (context-free) grammars such that $S_{i}$ does not occur on the right hand side of any production in $P_{i}$ for $i=1,2$ and suppose that $V_{1} \cap V_{2}=\emptyset$. Define a new grammar $G_{1} \otimes G_{2}=\left(\left(V_{1} \cup V_{2}\right)-\left\{S_{2}\right\}, \Sigma_{1} \cup \Sigma_{2}, P_{1} \cup P_{2}^{\prime}, S_{1}\right)$, where $P_{2}^{\prime}$ is $P_{2}$ with $S_{2}$ replaced by $S_{1}$. We say $G_{1} \oplus G_{2}$ is the direct sum of $G_{1}$ and $G_{2}$.

Note that we can always rename the alphabet of a grammar form without changing its grammatical family and moreover without any loss of generality we may assume its sentence symbol does not appear on the 
right hand side of any production. Therefore for any two grammar forms we can always define their direct sum.

Let us now state some straightforward but important results about grammar forms with respect to the operations defined above.

\section{Lemma 6.1}

Let $G_{i}=\left(V_{i}, \Sigma_{i}, P_{i}, S_{i}\right)$ fulfill the above conditions for $i=1,2$. Then

(i) $\mathcal{L}_{S}\left(G_{1} \oplus G_{2}, \Rightarrow\right)=\mathcal{L}_{s}\left(G_{1}, \Rightarrow\right) \forall \mathcal{L}_{s}\left(G_{2}, \Rightarrow\right), L\left(G_{1} \oplus G_{2}, \Rightarrow\right)$ $=L\left(G_{1}, \Rightarrow\right) \cup\left(G_{2}, \Rightarrow\right)$ and if $L$ is a coherent language in $\mathcal{Z}_{S}\left(G_{1} \oplus G_{2}, \Rightarrow\right)$ then $L$ is either in $\mathcal{X}_{s}\left(G_{1}, \Rightarrow\right)$ or in $\mathcal{Z}_{s}\left(G_{2}, \Rightarrow\right)$.

(ii) If $G_{i} \Delta \vec{S} G$ for some $G, i=1,2$, then $G_{1} \otimes G_{2} \underset{S}{\Delta} G$.

(iii) $\mathcal{L}_{S}(G, \Rightarrow)$ is closed under superdisjoint union for each grammar form $G$.

(iv) $m=\left\{\mathcal{L}_{\mathrm{S}}(G, \Rightarrow): G\right.$ is a context-free grammar form $\}$ is closed under superdisjoint wedge.

(v) $\mathcal{Z}_{s}(G, \Rightarrow)$ is closed under both breaking and extraction for each grammar form $G$.

(vi) $M$ is closed under extraction.

Proof: (i) Follows from the definitions, noting that if $G$ \& $G_{1} \theta G_{2}$ then $G$ can be decomposed into the direct sum of $G_{j}^{\prime}$ and $G_{2}^{\prime}$ such that $G_{j} \vec{s} G_{1}, G_{2}^{\prime} \vec{s} G_{2}$ and $G=G_{1}^{\prime} \otimes G_{2}^{\prime}$. The final statement follows directly from the notion of a coherent language.

(ii) Clear.

(iii) Let $L_{1}, L_{2}$ be arbitrary languages in $\mathcal{L}_{s}(G, \Rightarrow)$ over disjoint alphabets and $G_{j} \underset{S}{ } G$ be two grammars such that $L\left(G_{j}, \Rightarrow\right)=L_{i}$, $i=1,2$ and $G_{1}$ and $G_{2}$ fulfill the direct sum conditions. Then $G_{1} \oplus G_{2}$ is well-defined, $L\left(G_{1} \oplus G_{2} \Rightarrow\right)=L_{1} \otimes L_{2}$ and $G_{1} \oplus G_{2} \underset{s}{\triangle} G$ by $(i i)$.

(iv) Consider two arbitrary s-grammatical families $\mathcal{Z}_{\mathrm{s}}\left(\mathrm{G}_{1}, \Rightarrow\right.$ ) and $\mathcal{L}_{s}\left(G_{2}, \Rightarrow\right)$. We may assume by the previous remarks that $G_{1}$ and $G_{2}$ fulfill the direct sum conditions hence $Z_{s}\left(G_{1} \oplus G_{2}, \Rightarrow\right)=Z_{s}\left(G_{1}, \Rightarrow\right) \vee Z_{s}\left(G_{2}, \Rightarrow\right)$ by (i).

(v) Let $L_{1} \subseteq \Sigma_{1}^{*}$ be an arbitrary language in $\mathcal{L}_{\mathrm{S}}(G, \Rightarrow)$ and $\Sigma_{2} \subseteq \Sigma_{1}$. since s-grammatical families are closed under intersection with regular sets, $L_{2}=L_{1} \cap \Sigma_{2}^{*}$ is in $\mathcal{L}_{s}(G, \Rightarrow)$. Hence s-grammatical families are closed under breaking. Letting $L \subseteq \Sigma^{*}$ be an arbitrary language in $\mathcal{L}_{S}(G, \Rightarrow)$ and $i \geq 1$ be an integer then $L(i)=L a\left(\Sigma^{*}-\Sigma^{i}\right)$. Hence $s$-grammatical families are closed under extraction. 
(vi) Consider an arbitrary grammar form $G=(V, \Sigma, P, S)$ and an integer $i \geq 1$. Letting $\mathcal{L}=\mathcal{L}_{5}(G, \Rightarrow)$ then it should be clear that $\mathcal{Z}(i)=\mathcal{L}_{s}\left(G_{i}, \Rightarrow\right)$, where $G_{i}$ is obtained from $G$ by the usual intersection with regular sets construction, that is $L\left(G_{j}, \Rightarrow\right)=L(G, \Rightarrow) \cap\left(\Sigma^{*}-\Sigma^{j}\right)$.

\subsubsection{Denseness and s-grammatical Families}

We have now prepared the way for the main result of this section, namely $\mathcal{L}(R E G)$ is density forcing for s-gramatical families.

\section{Theorem 6.2}

Let $M$ be the collection of s-grammatical families and $\mathcal{Z}$ in $m$ be a family containing all the finite sets. Then $\mathcal{L}$ is density forcing.

proof: We have to show that if $\mathcal{L}_{1}$ and $\mathcal{L}_{2}$ are arbitrary families in $m$ with $\mathcal{L} \leq \mathcal{L}_{1} \underset{\mathcal{L}}{2}$, then there exists $\mathcal{L}_{3}$ in $\boldsymbol{L}_{\text {such that }}$

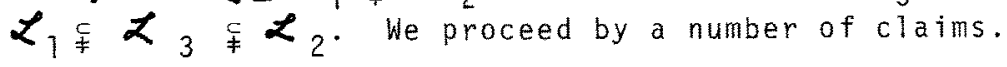

claim 1: $\mathcal{L}_{2}-\mathcal{L}_{1}$ contains an infinite coherent language $L$.

Proof of ctaim: clearly $\mathcal{X}_{2}-\mathcal{Z}_{1}$ contains only infinite languages, since both $\mathcal{Z}_{1}$ and $\mathcal{L}_{2}$ contain all the finite languages. Moreover since $\mathcal{Z}_{1 \neq} \mathcal{L}_{2}$ there must be at least one such language, say L. Let $L \subseteq \Sigma^{*}$ and assume $L$ is not coherent, since if it is coherent $L$ is the required language. Since $L$ is not coherent we can break it in a nontrivial way into $L_{1} \subseteq \Sigma_{1}^{*}$ and $L_{2} \subseteq \Sigma_{3}^{*}$ such that $\Sigma_{2}=\Sigma-\Sigma_{1}, L_{1} \cup L_{2}=L$ and hence $L_{1} \cup L_{2}=L$. Moreover $\Sigma_{1} \neq \Sigma \neq \Sigma_{2}$ and at least one of $L_{1}$ and $L_{2}$ must be infinite; let this be $L_{1}$. Now $\# \Sigma_{1}<\#$ by definition and letting $L_{T}$ be $L$ we can repeat this process if $L_{1}$ is not coherent. clearly this construction must terminate after a finite number of steps with an $L$ which is coherent. Furthermore the resulting $L$ must be in $\mathcal{Z}_{2}-\mathcal{Z}_{1}$ since s-grammatical families are closed under breaking.

claim 2: Let $L$ be a language in $\mathcal{L}_{2}-\mathcal{L}_{1}$, then there exists an integer $p \geq 1$ such that $L(p) \neq L$ and $L(p)$ is not in $\mathcal{L}_{1}$.

Proof of claim 2: Since $L$ is infinite there exist infinitely many values of $p$ such that $L(p) \neq L$. Now assume for each such $p$ that $L(p)$ is in $\mathcal{L}_{1}$. Let $G_{1}=\left(V_{1}, \Sigma_{1}, P_{1}, s_{1}\right)$ be a grammar form such that $\mathcal{L}_{s}\left(G_{1} \Rightarrow\right)=\mathcal{Z}_{1}$ and 1 et $\Delta$ be the alphabet of $L$. Now there are only 
finitely many dfl-substitutions mapping $\Sigma_{1}$ into the set of subsets of $\dot{\Delta}$. Since every $L(p) \neq L$ is in $\mathcal{L}_{1}$ and there are infinitely many such $L(p)$, then there are two distinct integers $m$ and $n$ such that $L(m)$ and $L(n)$ are obtained by the same dfl-substitution as far as terminals are concerned. Let $H_{m}$ and $H_{n}$ be the interpretations of $G_{1}$ generating $L(m)$ and $L(n)$ respectively. We may assume that the nonterminal alphabets of $H_{m}$ and $H_{n}$ are disjoint without any loss of generality. Now construct the sum of $H_{m}$ and $H_{n}$ as for the direct sum except that the terminal alphabets are identical and let $H$ be the resulting grammar. clearly $L(H, \Rightarrow)=L_{m} \cup L_{n}=L, H \underset{s}{\diamond} G_{1}$ and therefore $L$ is in $\mathcal{L}_{1}$. This is in contradiction to the assumption that $L$ is not in $\mathcal{L}_{1}$.

We are now able to establish the theorem by way of our final claim.

Craim 3: Let $L$ be a coherent language in $\mathcal{L}_{2}-\mathcal{L}_{1}, p \geq 1$ be an integer such that $L(p) \neq L$ and $L(p)$ is not in $\mathcal{Z}_{1}$ and $H_{p} \underset{s}{4} G_{2}$ has $L\left(H_{p}, \Rightarrow\right)=L(p)$. Then $\mathcal{Z}_{3}=\mathcal{L}_{s}\left(G_{1} \oplus H_{p} \Rightarrow\right)$ is strictly in between $\mathcal{L}_{1}$ and $\mathcal{L}_{2}$

Proof of Ctaim 3: First observe that we can always assume $G_{7} \oplus_{p} H_{p}$ is well defined, by suitably renaming the alphabet of $G_{1}$ if necessary. Second $\mathcal{L}_{1}=\mathcal{L}_{\mathrm{s}}\left(\mathrm{G}_{1} \Rightarrow\right) \subseteq \mathcal{L}_{3}$ follows from the definition of the direct sum. Proper inclusion follows since $L(p)$ is in $\mathcal{L}_{3}-\mathcal{L}_{1}$. Third, consider the relationship of $\mathcal{L}_{2}$ and $\mathcal{L}_{3}$. Consider an arbitrary language $L^{\prime}$ in $\mathcal{Z}_{3}$. L' can be expressed as $L^{\prime} \boldsymbol{U} L^{\prime \prime}$ where $L "$ is in $\mathcal{L}_{1}$ and $L^{\prime \prime \prime}$ is in $\mathcal{L}_{s}\left(H_{p}, \Rightarrow\right)$, since $\mathcal{L}_{3}=\mathcal{L}_{1} \vee \mathcal{L} \mathcal{L}_{s}\left(H_{p} \Rightarrow\right)$. However $L^{\prime \prime}$ and $L^{\prime \prime}$ are both in $\mathcal{Z}_{2}$, since $\mathcal{Z}_{1} \subseteq \mathcal{Z}_{2}$ and $H_{p} \Delta \vec{s}_{2} G_{2}$ implies $\mathcal{Z}_{\mathrm{s}}\left(\mathrm{H}_{\mathrm{p}}, \Rightarrow\right) \subseteq \mathcal{Z}_{2}$. Hence $L^{\prime \prime} \boldsymbol{U} L^{\prime \prime \prime}=L^{\prime}$ is in $\mathcal{Z}_{2}$ because s-grammatical families are closed under superdisjoint union (Lemma 6.1). Thus we have shown that $\mathcal{L}_{3} \subseteq \mathcal{Z}_{2}$. Proper inclusion follows from Lemma 6.1 (i) since $L$ is coherent and is neither in $\mathcal{L}_{1}$ nor in $\mathcal{L}_{s}\left(H_{p}, \Rightarrow\right)$.

This completes the proof of the theorem.

While we conjecture that the condition that $\mathcal{L}$ contain a 11 finite languages is a necessary and sufficient condition for $\mathcal{Z}$ to be density forcing in $M$ this remains an open question. Assuming this conjecture to be true implies that $m(\mathcal{L}(R E G), \mathcal{L}(C F))$ forms a maximal dense pair with respect to $\boldsymbol{M}$. In other words there is no $\mathcal{L} \neq \mathcal{Z}(\mathrm{REG})$ such that $m(\mathcal{L}, \mathcal{L}(C F))$ is a dense pair. 
We now show that the problem of maximality for $m$, the collection of $\mathrm{s}$-grammatical families, is reducible to a particular question about finite forms.

Consider any grammar form $F_{1}$ such that $\mathcal{L}_{s}\left(F_{1} \Rightarrow\right) \nsubseteq \mathcal{L}(R E G)$. Letting the terminal alphabet of $F_{1}$ be $\left\{a_{1}, \ldots, a_{n}\right\}, n>0$, then by Lemma 4.22 we know that there are positive integers $k_{i}, i=1, \ldots, n$ such that:

$a_{i}^{k_{i}}$ is not in $L\left(F_{1}, \Rightarrow\right)$, for $i=1, \ldots, n$.

Let $k=\max \left(\left\{k_{i}: 1 \leq i \leq n\right\}\right)+1$ and add to $F_{1}$ new nonterminals and right linear productions generating the language

$$
\left\{a_{1}, \ldots, a_{n}\right\} *-\left\{a_{1}^{k}\right\}_{1}^{k_{1}}, \ldots, a_{n}^{k} n_{\}},
$$

this can always be done since we may assume the sentence symbol of $F_{1}$ does not appear on the right hand side of any production.

Let the resulting grammar form be denoted by $F$. Clearly

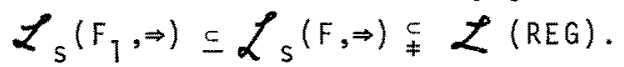

Now let $d, d_{1}, \ldots, d_{n+1}$ be new terminal symbols and let

$\Sigma=\left\{d_{1}, \ldots, d_{n+1}\right\}$. Define the finite forms $D_{1}$ and $D_{2}$ by:

$$
\begin{aligned}
& L\left(D_{1}, \Rightarrow\right)=\Sigma^{k} l \cup \ldots \cup \Sigma^{k} n-\left\{d_{i}^{k}: 1 \leq i \leq n+1,1 \leq j \leq n\right\} \text { and } \\
& L\left(D_{2}, \Rightarrow\right)=\left\{d^{k} j: 1 \leq j \leq n\right\} .
\end{aligned}
$$

For finite forms it is easy to show that the language of the form completely specifies the form as far as its language family is concerned. Consider $F \oplus D_{1}$ and $F \oplus D_{2}$. clearly $\mathcal{L}_{5}(F, \Rightarrow) \subseteq \mathcal{L}_{s}\left(F \oplus D_{1}, \Rightarrow\right) \subseteq$

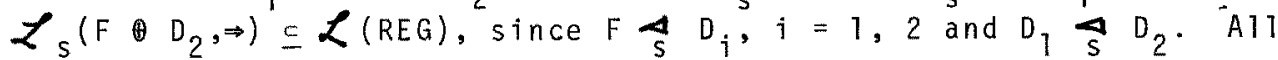
these containments are proper if we assume that at least one $k_{i}$ is different from one. For considering each of them in turn we have:

(a) $L\left(D_{\eta}, \Rightarrow\right)$ is not in $\mathcal{Z}_{s}(F, \Rightarrow)$. For assume otherwise, in which case there is an $F^{\prime} \underset{s}{S} F(\mu)$, for some $\mu$ such that $L\left(F^{\prime}, \Rightarrow\right)=L\left(0_{1}, \Rightarrow\right)$. However this means $\mu$ maps $\left\{a_{1}, \ldots, a_{n}\right\}$ onto $\left\{d_{1}, \ldots, d_{n+1}\right\}$, that is there exists an $a_{i}$ with $\# \mu\left(a_{i}\right) \geq 2$. Without loss of generality assume that $d_{1}$ and $d_{2}$ are in $\mu\left(a_{i}\right)$. Now there is a word $x$ in $\left\{d_{1}, d_{2}\right\} * n L\left(D_{1}, \Rightarrow\right)$ such that $|x|=k_{j}$ and hence $\mu^{-1}(x)=a_{i}{ }^{k}$ is in $L(F, \Rightarrow)$. This is a contradiction, hence $\mathcal{L}_{S}\left(F, \Rightarrow \underset{\ddagger}{c} \mathcal{L}_{\mathrm{s}}\left(F \oplus D_{1}, \Rightarrow\right)\right.$.

(b) Observe that $L\left(D_{2}, \Rightarrow\right)$ is not in $\mathcal{L}_{s}\left(F \otimes D_{1} \Rightarrow\right)$, since it is not in $\mathcal{L}_{s}(F, \Rightarrow)$ and $i t$ is not in $\mathcal{L}_{s}\left(D_{1}, \Rightarrow\right)$. Therefore $\mathcal{L}_{\mathrm{s}}\left(\mathrm{F} \oplus \mathrm{D}_{1}, \Rightarrow\right) \nsubseteq \mathcal{L}_{\mathrm{s}}\left(\mathrm{F} \oplus \mathrm{D}_{2}, \Rightarrow\right)$.

(c) $\mathcal{L}_{s}^{s}\left(F \oplus D_{2}, \Rightarrow\right)$ c $\mathcal{L}^{s}(R E G)$ since $a^{+}$is neither in $\mathcal{X}_{s}(F, \Rightarrow)$ nor in $\mathcal{L}_{s}^{s}\left(D_{2}, \Rightarrow\right)$. 
In the case that $k_{1}=k_{2}=\ldots=k_{n}=1$ we have $L(F, \Rightarrow)=\left\{a_{1}, \ldots, a_{n}\right\} *-\left\{a_{1}, \ldots, a_{n}\right\}, L\left(D_{1}, \Rightarrow\right)=\emptyset$ and $L\left(D_{2}, \Rightarrow\right)=\{d\}$. Thus $\mathcal{L}_{s}(F, \Rightarrow)=\mathcal{L}_{s}\left(F \oplus D_{1}, \Rightarrow\right.$ but $\mathcal{L}_{s}(F, \Rightarrow)=\mathcal{L}_{s}\left(F \oplus D_{1}, \Rightarrow\right)$ $\mathcal{L}_{\mathrm{s}}\left(\mathrm{F} \oplus \mathrm{D}_{2}, \Rightarrow\right) \underset{\mathcal{L}}{\mathrm{L}}(\mathrm{REG})$.

However in the following construction it is of no consequence whether or not $\mathcal{L}_{s}(F, \Rightarrow)$ is properly contained in $\mathcal{L}_{s}\left(F \otimes 0_{1}, \Rightarrow\right)$. We proceed by defining two finite forms $H_{1}$ and $H_{2}$ obtained by taking all words of length $\leq k$ from $L\left(F \otimes D_{1}, \Rightarrow\right)$ and $L\left(F \otimes D_{2} \Rightarrow\right)$ respectively. We have the proper inclusion:

$$
\mathcal{L}_{\mathrm{s}}\left(\mathrm{H}_{1}, \Rightarrow\right) \underset{\ddagger}{\subsetneq}\left(\mathrm{H}_{2}, \Rightarrow\right) \text {, }
$$

since $L\left(F \oplus D_{1}, \Rightarrow\right)$ and $L\left(F \oplus D_{2}, \Rightarrow\right)$ agree for all words of length $>k$. Letting $F^{(k)}$ be the finite form obtained from $F$ by taking all words of length $\leq k$ from $L(F, \Rightarrow)$, then $H_{i}=F(k) \oplus D_{i}, i=1,2$. Note that proper inclusion always holds; even for the exceptional case. We now have the following preliminary lemma.

Lemma 6.3

If the pair $\left(\mathcal{L}_{\mathrm{s}}\left(\mathrm{H}_{1}, \Rightarrow\right), \mathcal{K}_{\mathrm{s}}\left(\mathrm{H}_{2}, \Rightarrow\right)\right.$ is not dense then the pair $\left(\mathcal{Z}_{\mathrm{s}}\left(\mathrm{F} \oplus \mathrm{D}_{1}, \Rightarrow\right), \mathcal{L}_{\mathrm{s}}\left(\mathrm{F} \oplus \mathrm{D}_{2}, \Rightarrow\right)\right)$ is not dense.

Proof: By the assumption of the lemma there are two forms $E_{1}$ and $E_{2}$ such that

$$
\mathcal{L}_{s}\left(H_{1}, \Rightarrow\right) \subseteq \mathcal{L}_{s}\left(E_{1}, \Rightarrow\right) \subseteq \mathcal{L}_{s}\left(E_{2}, \Rightarrow\right) \subseteq \mathcal{L}_{s}\left(H_{2}, \Rightarrow\right)
$$

and moreover $E_{2}$ is a successor of $E_{1}$.

We claim that $E_{j}=F^{(k)} \oplus E_{i}, i=1,2$, where

$\mathcal{L}_{\mathrm{s}}\left(D_{1}, \Rightarrow\right) \subseteq \mathcal{L}_{\mathrm{s}}\left(E_{1}^{\prime}, \Rightarrow\right) \underset{\mathcal{L}_{\mathrm{s}}}{ }\left(\mathrm{E}_{2}^{\prime}, \Rightarrow\right) \subseteq \mathcal{L}_{\mathrm{s}}\left(\mathrm{D}_{2}, \Rightarrow\right)$.

Consider $L\left(E_{2}, \Rightarrow\right)$. It can be written as

$$
L\left(E_{2}, \Rightarrow\right)=L\left(F(k)^{2} \Rightarrow\right) \cup\left(E_{2}^{\prime}, \Rightarrow\right) \text {, }
$$

where $F^{(k)} \&_{S} F^{(k)}$ and $E_{2} \underset{s}{ } D_{2}$. But we also have:

$$
F^{(k)} \oplus D_{1} \otimes F^{(k)} \oplus E_{2}^{\prime} .
$$

Now $L\left(D_{1} \Rightarrow\right)$ is not in $\mathcal{L}_{s}(F, \Rightarrow)$ unless $L\left(D_{1}, \Rightarrow\right)=\emptyset$. In either case we have $D_{1} \Delta E_{2}^{\prime}$. Since $L(F(k) \Rightarrow)=\left\{a_{1}, \ldots, a_{n}\right\}^{\leq k}-\left\{a_{i} k_{i}: 1 \leq i \leq n\right\}$, is not in $\mathcal{X}_{\mathrm{S}}\left(D_{2} \Rightarrow\right)$ and hence not in $\mathcal{Z}_{\mathrm{s}}\left(\mathrm{E}_{2}^{\prime}, \Rightarrow\right)$. Moreover $L\left(F(k), \Rightarrow\right.$ is clearly coherent, therefore $L(F(k), \Rightarrow)$ is in $\mathcal{L}_{s}(F(k), \Rightarrow)$. But this implies $F(k) \rightarrow \bar{F}(k) \rightarrow F(k)$. Thus we have demonstrated that $E_{2}=F(k) \oplus E_{2}^{\prime}$, where $D_{1} \underset{s}{s} E_{2}^{\prime} \stackrel{s}{s} D_{2}$.

By a similar argument we obtain

$$
E_{1}=F^{(k)} \odot E_{1}^{\prime} \text {, }
$$


128

where $D_{1} \underset{S}{A} E_{j} \underset{S}{\Delta} D_{2}$. Since $E_{1} \underset{S}{\rightarrow} E_{2}$ we also have $E_{1}^{\prime} \underset{s}{A} E_{2}^{\prime}$ and hence

$$
\mathcal{L}_{\mathrm{s}}\left(D_{1}, \Rightarrow\right) \subseteq \mathcal{L}_{\mathrm{s}}\left(E_{1}^{\prime}, \Rightarrow\right) \subseteq \mathcal{L}_{5}\left(E_{2}^{\prime}, \Rightarrow\right) \subseteq \mathcal{L}_{\mathrm{s}}\left(D_{2}, \Rightarrow\right) \text {. }
$$

since $\mathcal{L}_{s}\left(F^{(k)} \oplus E_{1}^{\prime} \Rightarrow\right) \subsetneq \mathcal{Z}_{s}\left(F(k) \oplus E_{2}^{\prime} \Rightarrow\right)$ we conclude that $\mathcal{L}_{\mathrm{s}}\left(E_{1} \Rightarrow\right) \underset{f}{\not}\left(E_{2}, \Rightarrow\right)$.

Moreover we claim that $E_{2}^{\prime}$ is a successor of $E_{j}^{\prime}$. For if this is not the case there is a form $E_{3}^{\prime}$ such that $\mathscr{L}_{s}\left(E_{j}, \Rightarrow\right) \underset{\ddagger}{\mathcal{L}_{s}}\left(E_{3}^{\prime}, \Rightarrow\right) \varsubsetneqq$ $\mathscr{L}_{\mathrm{s}}\left(E_{2}^{\prime} \Rightarrow\right)$ and this implies that the form $F^{(k)} \theta_{E_{3}^{\prime}}$ lies properly in between $\mathcal{L}_{\mathrm{s}}\left(E_{1} \Rightarrow\right)$ and $\mathcal{L}_{\mathrm{s}}\left(E_{2}, \Rightarrow\right)$, a contradiction.

Having established the claim we now proceed with the lemma by defining:

$$
G_{i}=F \oplus E_{i}^{\prime}, i=1,2 \text {. }
$$

This gives

$$
\mathcal{L}_{s}\left(F \otimes D_{1}, \Rightarrow\right) \subseteq \mathcal{L}_{s}\left(G_{1}, \Rightarrow\right) \underset{\mathcal{L}_{s}}{ }\left(G_{2}, \Rightarrow\right) \subseteq \mathcal{L}_{s}\left(F \otimes D_{2}, \Rightarrow\right)
$$

That $G_{2}$ is a successor of $G_{1}$ follows by establishing the following claim.

Claim: Let $G$ be a grammar form satisfying

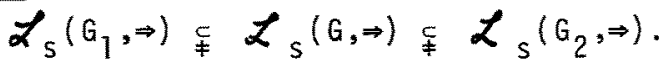

Then there is a grammar form $G(k)$ satisfying

$$
\mathcal{Z}_{s}\left(F^{(k)} \oplus E_{j}^{\prime}, \Rightarrow\right) \subsetneq \mathcal{Z}_{s}\left(G(k), \Rightarrow \subseteq \mathcal{Z}_{s}\left(F^{(k)} \oplus E_{2}^{\prime}, \Rightarrow\right)\right. \text {. }
$$

Proof of claim: It is convenient to introduce some auxiliary notation. For a language $L$ and a positive integer $t$, we denote by $L_{\leq t}\left(L_{>t}\right)$ the subset of $L$ consisting of all words of length $\leq t(>t)$.

For a grammar form $H$, the families $\mathcal{L}_{s}(H, \Rightarrow)_{s t}$ and $\mathcal{Z}_{s}(H, \Rightarrow)_{>t}$ are defined by:

$$
\mathcal{L}_{s}(H, \Rightarrow)_{\leq t}=\left\{L_{\leq t}: L \text { in } \mathcal{L}_{s}(H, \Rightarrow)\right\}
$$

and

$$
\mathcal{L}_{s}(H, \Rightarrow)_{>t}=\left\{L_{>t}: L \text { in } \mathcal{L}_{s}(H, \Rightarrow)\right\}
$$

For the $G_{1}, G$ and $G_{2}$ of the claim we have:

$$
\mathcal{L}_{\mathrm{s}}\left(G_{1}, \Rightarrow\right)_{>k}=\mathcal{L}_{\mathrm{s}}(G, \Rightarrow)_{>k}=\mathcal{L}_{\mathrm{s}}\left(G_{2}, \Rightarrow\right)_{>k} \text {. }
$$

It is also clear that:

$$
\mathcal{L}_{\mathrm{s}}\left(\mathrm{G}_{1}, \Rightarrow\right)_{\leq k} \subseteq \mathcal{Z}_{\mathrm{s}}(\mathrm{G}, \Rightarrow)_{\leq k} \subseteq \mathcal{L}_{\mathrm{s}}\left(\mathrm{G}_{2}, \Rightarrow\right)_{s k} \text {. }
$$

Now assume the claim is false, in other words either

$$
\mathcal{L}_{s}\left(G_{1}, \Rightarrow\right)_{\leq k}=\mathcal{L}_{s}(G, \Rightarrow)_{\leq k} \text { or } \mathcal{L}_{s}(G, \Rightarrow)_{\leq k}=\mathcal{L}_{s}\left(G_{2}, \Rightarrow\right)_{s k} \text {. }
$$

We will only consider the first alternative since the second can be dealt with analogously.

Thus $\mathcal{L}_{s}(G, \Rightarrow)_{\leq k}=\mathcal{L}_{s}\left(G_{1}, \Rightarrow\right)_{\leq k}=\mathscr{L}_{s}\left(F(k) \oplus E_{1}^{\prime} \Rightarrow\right)$ and because 
we have both $\mathcal{L}_{s}\left(G_{1}, \Rightarrow\right)_{>k}=\mathcal{L}_{s}(G, \Rightarrow)_{>k}$ and $\mathcal{L}_{s}\left(G_{1}, \Rightarrow\right)_{\leq k}=\mathcal{L}_{s}(G, \Rightarrow)_{s k}$ the inclusion $\mathscr{Z}_{S}\left(G_{1}, \Rightarrow\right) \subseteq \mathcal{Z}_{S}(G, \Rightarrow)$ can be proper only if some combination of the "high" and "low" languages is possible in the family $\mathcal{L}_{\mathrm{s}}(G, \Rightarrow)$, which is not possible in the family $\mathcal{L}_{\mathrm{s}}\left(G_{1}, \Rightarrow\right)$. However, this contradicts the definition of $G$ and the choice of $k$, because of the direct sum no new dependencies can be created in $\mathcal{L}_{s}(G, \Rightarrow)$. That is $\mathcal{L}_{S}(G, \Rightarrow)=\mathcal{L}_{s}\left(G_{1}, \Rightarrow\right)$, a contradiction. Thus the claim has been established.

The lemma now follows since $G_{2}$ is a successor of $G_{1}$ and hence the pair $\left(\mathcal{L}_{s}(F, \Rightarrow), \mathcal{L}(R E G)\right.$ is not dense.

This lemma immediately yields the following "reduction" theorem.

\section{Theorem 6.4}

The pair $\left(\mathcal{Z}_{\mathrm{s}}\left(\mathrm{H}_{1}, \Rightarrow\right), \mathcal{L}_{\mathrm{s}}\left(\mathrm{H}_{2}, \Rightarrow\right)\right)$ is dense iff the pair $\left(\mathcal{L}_{\mathrm{s}}\left(F \otimes D_{1}, \Rightarrow\right), \mathcal{L}_{\mathrm{s}}\left(\mathrm{F} \oplus \mathrm{D}_{2}, \Rightarrow\right)\right)$ is dense.

Proof: if: Assume the pair $\left(\mathcal{Z}_{\mathrm{S}}\left(\mathrm{H}_{1}, \Rightarrow\right), \mathcal{L}_{\mathrm{s}}\left(\mathrm{H}_{2}, \Rightarrow\right)\right)$ is dense, then for all $\mathrm{H}_{3}$ and $\mathrm{H}_{4}$ such that

$$
\mathcal{L}_{\mathrm{s}}\left(\mathrm{H}_{1} \Rightarrow\right) \subseteq \mathcal{L}_{\mathrm{s}}\left(\mathrm{H}_{3}, \Rightarrow\right) \subsetneq \mathcal{L}_{\mathrm{s}}\left(\mathrm{H}_{4}, \Rightarrow\right) \subseteq \mathcal{L}_{\mathrm{s}}\left(\mathrm{H}_{2}, \Rightarrow\right)
$$

there is an $\mathrm{H}_{5}$ with

$$
\mathcal{L}_{s}\left(H_{3}, \Rightarrow\right)_{\ddagger}^{c} \mathcal{L}_{s}\left(H_{5}, \Rightarrow\right) \underset{\ddagger}{\subsetneq} \mathcal{L}_{s}\left(H_{4}, \Rightarrow\right) \text {. }
$$

Now since $H_{i}=F(k) \oplus D_{i}, i=1,2$, we must have

$$
H_{i}=F^{(k)} \oplus C_{i}, i=3,4,5
$$

by similar arguments to those used in the proof of Lemma 6.3 , where $D_{1} \underset{s}{\Delta} C_{i} \underset{s}{\Delta} D_{2}, i=1,2,3$ and $C_{1} \underset{s}{\Delta} C_{3} \underset{s}{\Delta} C_{2}$. Thus we have

$$
\begin{aligned}
& \mathcal{L}_{s}\left(F \oplus D_{1}, \Rightarrow\right) \subseteq \mathcal{L}_{s}\left(F \oplus c_{1}, \Rightarrow\right) \subseteq \mathcal{L}_{s}\left(F \oplus c_{3}, \Rightarrow\right) \subseteq \mathcal{L}_{s}\left(F \oplus c_{2}, \Rightarrow\right) \subseteq \\
& \mathscr{L}_{s}\left(F \oplus D_{2}, \Rightarrow\right) .
\end{aligned}
$$

Moreover $\mathcal{L}_{\mathrm{s}}\left(\mathrm{F} \oplus \mathrm{c}_{1}, \Rightarrow\right) \underset{\mp}{\mathcal{L}_{5}}\left(\mathrm{~F} \oplus \mathrm{c}_{3}, \Rightarrow\right) \underset{\mp}{\mathcal{L}_{\mathrm{s}}}\left(\mathrm{F} \oplus \mathrm{C}_{2}, \Rightarrow\right)$, since $\mathcal{L}_{s}\left(F(k) \oplus c_{1} \Rightarrow\right) \subsetneq \mathcal{L}_{s}\left(F(k) \oplus c_{3}, \Rightarrow\right) \subsetneq \mathcal{L}_{s}\left(F \oplus c_{2}, \Rightarrow\right)$. In other words the pair $\left(\mathcal{L}_{s}\left(F \oplus D_{1}, \Rightarrow\right), \mathcal{L}_{s}\left(F \otimes D_{2}, \Rightarrow\right)\right)$ is dense.

only if: Assume the pair $\left(\mathcal{L}_{s}\left(F \oplus D_{1}, \Rightarrow\right), \mathcal{L}_{s}\left(F \oplus D_{2}, \Rightarrow\right)\right)$ is dense and the pair $\left(\mathcal{L}_{\mathrm{s}}\left(\mathrm{H}_{7}, \Rightarrow\right), \mathcal{K}_{\mathrm{s}}\left(\mathrm{H}_{2}, \Rightarrow\right)\right)$ is not dense. Then Lemma 6.3 provides a contradiction.

This completes the theorem. 
Now the pair $(\mathscr{L}(R E G), \mathcal{L}(C F))$ is maximal dense iff there is no sub-regular grammatical family $\mathscr{L}$ such that the pair $(\mathcal{L}, \mathscr{L}(R E G))$ is dense. Although we have not settled this question we have reduced it via the above theorem to the problem of the denseness of a pair of specific finite forms.

The techniques developed in this section to establish the denseness of the pair $(\mathcal{L}(R E G), \mathcal{L}(C F))$ are inapplicable if we restrict our attention to the collection of two-symbol s-grammatical families. Thus section 4 is devoted to establishing the denseness of this latter collection by use of alternative techniques.

\section{II.6.3 MSW Spaces}

Theorem 6.2 can be considerably generalized by considering arbitrary collections of language families satisfying certain basic properties necessary for proving a result akin to it; such collections are termed MSW spaces. After defining an MSW space we will prove the analogue of Theorem 6.2 and then show how an arbitrary collection can be turned into an MSW space in a particularly simple manner. This demonstrates not only that such spaces are easily obtained, but also that the abstraction is meaningful in that "most" MSW spaces are not generated by grammar forms.

A collection of $M$ of language families is an MSW-space if it satisfies the following three conditions:

(i) Each $\mathcal{L}$ in $M$ is closed under superdisjoint union and breaking. (ii) $M$ is closed under superdisjoint wedge.

(iii) For each infinite language $L$ occurring in some language family of $M$ there exist subsets $L_{i}$ of $L$ for $i=1,2, \ldots$ such that

(a) and (b) hold:

(a) L is in a language family $\not{\not}$ of $M$ iff $L_{j}$ is in $\mathcal{L}$ for all $i$ with $L_{i} * L$,

(b) If $L$ belongs to $\mathcal{Z}$ in $M$, then for every $i$ with $L_{i} \neq L$ there exists an $\mathcal{L}_{i}$ in $M$ such that $\mathcal{L}_{i} \leq \mathcal{L}, L_{i}$ is in $\mathcal{L}_{i}$ and $L$ is not in $\mathcal{L}_{i}$.

Corozzary 6.5

$M$, the collection of s-grammatical families is an MSW space.

Exoof: Conditions (i) and (ii) are contained in Lemma 6.1. Consider condition ( $i j i$ ). Let $L_{i}=L(i)$ for $i \geq 1$, then essentially condition $(i j, a)$ has been proved under $\mathrm{Claim} 2$ of Theorem 6.2. Similarly $\mathcal{L}_{i}$ 
of condition ( $\left.i i_{i, b}\right)$ is defined by $H_{i}$ in $\mathrm{Claim} 3$ of the proof of Theorem 6.2. Assuming $H \underset{s}{A} G_{2}$ is a grammar form with $L(H, \Rightarrow)=L$ and $H_{j}$ is the grammar form fulfilling $L\left(H_{j} \Rightarrow\right)=L n\left(\Delta^{*}-\Delta^{j}\right)$ when $L \subseteq \Delta^{*}$, then $\mathcal{Z}_{s}\left(H_{i} \Rightarrow\right)$ is the extraction of $\mathcal{L}_{s}(H, \Rightarrow)$ with respect to i. Clearly $L$ is not in $\mathcal{Z}_{s}\left(H_{i}, \Rightarrow\right)$ if $L \neq L(i)$, since words of length $i$ do not appear in languages in $\mathcal{Z}_{s}\left(H_{i}, \Rightarrow\right)$.

It is now possible to generalize Theorem 6.2 considerably, namely:

\section{Theorem 6.6}

Let $M$ be an MSW space and let $\mathcal{F}$ be the collection of all finite languages occurring in language families of $m$. If $\mathcal{L}$ is any family of $M$ containing $\mathcal{J}$, then $\mathcal{Z}$ is density forcing.

Proof: This is left to the reader.

The notion of an MSW space together with Theorem 6.6 enables many "dense" families to be exhibited. Letting $\boldsymbol{M}$ be a collection of language families, denote by $M(i)$ for $i \geq 1$, the collection $\{\mathcal{L}(i): \mathcal{L}$ is in $\mathbb{Z}\}$.

Let $\mathcal{F}$ denote the collection of all finite languages, $\boldsymbol{M}_{1}$ be the collection of all context-free s-grammatical families, $\boldsymbol{M}_{2}$ the collection of all $\mathrm{s}$-grammatical families (see section IV.1), $m_{3}$ the collection of all linear s-grammatical families and $\boldsymbol{m}_{4}$ the collection of all synchro-EOL grammatical families (see section III.2). The following corollary can easily be shown.

\section{Corotzary 6.7}

For a $11 i, 1 \geq j \geq 4$, for all $j \geq 1$, if $\mathcal{L}$ is a language family in $M_{i}(j)$ containing $\mathcal{F}(j)$ then $\mathcal{L}$ is density forcing with respect to $m_{j}(j)$.

We now turn to the problem of "constructing" MSW spaces. First we establish an "invariance" theorem concerning closure under superdisjoint wedge and extraction.

\section{Theorem 6.8}

Let $M$ be a collection of language families such that each family $\mathcal{L}$ of $M$ is closed under superdisjoint union, intersection with 
regular sets and covering. Let $\boldsymbol{W}$ be the closure of $\mathbb{W}$ under superdisjoint wedge and extraction. Then each $\mathcal{L}$ in $\boldsymbol{N}$ is closed under superdisjoint union, intersection with regular sets and covering and $\eta$ is in MSW space.

Pxoof: We first show that each $\mathcal{Z}$ in $\boldsymbol{n}$ has the specified closure properties. Consider two arbitrary language families $\mathcal{L}_{1}$ and $\mathcal{L}_{2}$ in $\mathbb{W}$. By the assumptions of the theorem both $\mathcal{\alpha}$, and $\mathcal{L}{ }_{2}$ have the desired closure properties. We first establish the effect of closure under superdisjoint wedge by demonstrating that $\mathcal{L}=\mathcal{L}_{1} \dot{\vee}_{2}$ has the desiredclosure properties, that is these closure properties are invariant under superdisjoint wedge.

Now each $L$ in $\mathcal{L}$ can be expressed as $L=L_{1} \dot{v} L_{2}$ by the definition of superdisjoint wedge (recall that $L_{1}$ and $L_{2}$ are over disjoint alphabets).

(i) Closure under $\boldsymbol{U}$ : Consider arbitrary languages $L$ and $M$ in $\mathcal{L}$, where $L=L_{1} \cup L_{2}$ and $M=M_{1} \cup M_{2}$ with $L_{i}$ and $M_{i}$ in $\mathcal{L}_{i}, i=1,2$. We need to show that $L \dot{B} M$ is in $\mathcal{L}$. This implies $L_{1}, L_{2}, M_{1}$ and $M_{2}$ are over pairwise disjoint alphabets. Now $L \dot{U} M=\left(L, \forall L_{2}\right) \cup\left(M_{1} \cup M_{2}\right)$, hence this can be expressed as $\left(L_{1} \cup M_{1}\right) \cup\left(L_{2} \cup M_{2}\right)$, and because $L_{i} \cup M_{i}$ is in $\mathcal{L}_{i}$, $i=1,2$, then $L$ U $M$ is in

(ii) Closure under $n R$ : Consider an arbitrary regular set $R$, then $L \cap R=(L, \cap R) \cup\left(L_{2} \cap R\right)$ and since $\mathscr{L}_{1}$ and $\mathscr{L}_{2}$ are ciosed under intersection with regular sets, $L_{i} \cap R$ is in $\mathcal{L} i$, $i=1,2$. Hence $L \cap R$ is in $\mathcal{L}$.

(iii) closure under covering: We need to show that $\mathcal{L}$ is closed under covering in order to demonstrate the invariance of the three closure properties under superdisjoint wedge closure. Again consider an arbitrary $L \subseteq \Sigma^{*}$ such that $L(i) \neq L$ is in $\mathcal{Z}$ for infinitely many $i$. We need to show that' $L$ is in $\mathcal{L}$. clearly, for al1 $i, L(i)=L_{1, i} \cup L_{2, i}$ with $L_{j, 1}$ in $Z_{j}, j=1,2$. First observe that there are only a finite number of partitions of $\Sigma$ into $\Sigma_{1} \cup \Sigma_{2}$. Hence there is one partition $\Sigma=\Sigma_{1} \dot{U} \Sigma_{2}$ say, such that for infinitely many $i$ we have:

$L(i)=L_{1, i} \cup L_{2, i}$ where $L_{1, i} \subseteq \Sigma_{1}^{*}$ and $L_{2, i} \subseteq \Sigma_{2}^{*}$, that is where $L_{1, i}=M_{1}(i)$ with $M_{1}=L n \sum_{i}^{*}$ and similarly $L_{2, i}=M_{2}(i)$ with $M_{2}=L \cap \Sigma_{2}^{\star}$.

Since for infinitely many $i, M_{j}(i)$ is in $\mathcal{L}_{j}$, for $j=1,2$ then $M_{j}$ is in $\mathcal{L}_{j}$, for $j=1,2$ since $\mathcal{L}_{1}$ and $\mathcal{L}_{2}$ are closed under covering. But $L=M_{1} \cup M_{2}$ hence $L$ is in $\mathcal{L}$ as claimed. 
In the second part of the proof we consider the effect of extraction closure on the three closure operations. Thus for each $\mathcal{Z}$ in $m$ we need to show that for all $p>0, \mathcal{K}(p)$ satisfies the required closure properties.

(iv) closure under $\boldsymbol{\theta}$ : Consider two languages $L$ and $M$ in $\mathcal{L}(p)$. Now $L=L^{\prime}(p)$ and $M=M^{\prime}(p)$ for some $L^{\prime}$ and $M^{\prime}$ in $\mathcal{L}$. Since we wish to form $L \cup M$ we may assume that $L$ and $M$ are over disjoint alphabets, that is $L^{\prime}(p)$ and $M^{\prime}(p)$ are over disjoint alphabets. Since $\mathcal{L}$ is closed under intersection with regular sets, then not only are $L^{\prime}$ and $M^{\prime}$ in $\mathcal{L}^{\prime}$ but also $L^{\prime}(p)$ and $M^{\prime}(p)$ are in $\mathcal{L}$. Thus $L^{\prime}(p) \cup M^{\prime}(p)$ is in $\mathcal{L}$ and hence in $\mathcal{L}(p)$ as desired.

(v) Closure under $n R$ : Consider a language $L$ in $\mathcal{L}(p)$ and a regular language $R$. Now $L=L^{\prime}(p)$ for some $L^{\prime}$ in $\mathcal{L}$, $L \cap R=\left(L^{\prime} \cap R\right)(p)$ and the result follows.

(vi) Closure under covering: Consider an arbitrary language $L$ such that $L(i) \neq L$ is in $\mathcal{L}(p)$ for infinitely many $i$. Now $L(i)=L_{i}(p)$ for some $L_{i}$ in $\mathcal{L}$, where $L_{i}$ may or may not contain words of length $p$. However since $\mathcal{L}$ is closed under intersection with regular sets $L_{i}(p)$ is also in $\mathcal{L}$, that is $L(i)$ is in $\mathcal{L}$ for infinitely many $i$. But this implies that $L$ is in $\mathcal{X}$ and hence $L(p)$ is in $\mathcal{L}(p)$ as desired.

Finally we need to show that $\boldsymbol{n}$ is indeed an MSW space. But this follows immediately from the construction of $\pi$.

\section{Corozzary 6.9}

Consider an arbitrary family $\mathcal{L}$ of languages and the collection $\boldsymbol{K}=\{\{L\}: L$ is in $\mathcal{L}\}$. close each language family $\{L\}$ in $\mathcal{H}_{\mathrm{f}}$ with respect to the operations of $\boldsymbol{U}$, intersection with regular sets and covering yielding a collection $m$ of language families. close $M$ under superdisjoint wedge and extraction to abtain $\eta$. Then $\eta$ is an MSW space.

\section{Coroltary 6.10}

Let $\eta$ be the collection of all language families consisting of context-free languages, such that each $\mathcal{\alpha}$ in $\eta$ is closed under $\dot{\boldsymbol{V}}$, intersection with regular sets and covering.

Then $\boldsymbol{\eta}$ is an MSW space.

Proof: The closure of $\eta$ under $\boldsymbol{V}$ and extraction is $\boldsymbol{\eta}$ itself. 
Let $\&$ be an arbitrary family of languages closed under union and intersection with regular sets. Let $\eta$ be the collection of all subsets $\mathcal{L}$ of $\boldsymbol{S}$ which are closed under $\dot{U}$, intersection with regular sets and covering.

Then $\eta$ is an MSW space.

Proof: By Theorem 6.8 it suffices to show that $\eta$ is closed under $\dot{\forall}$ and extraction. Consider two families $\mathcal{L}_{1}$ and $\mathcal{L}_{2}$ in $\eta$. Since $\mathcal{L}_{1} \dot{\vee} \mathcal{L}_{2}=\left\{L_{1} \cup L_{2}: L_{i}\right.$ in $\left.\mathcal{L}_{i}, i=1,2\right\}, \mathcal{L}_{1}, \mathcal{L}_{2} \subseteq \xi$ and $\mathcal{L}_{1} \vee \mathcal{L}_{2} \leq \mathcal{Q}$ we have $\mathcal{L}_{1} \dot{\nabla} \mathcal{L}_{2} \leq S$. However since $\mathcal{L}_{1}$ and $\mathcal{L}_{2}$ are closed under $\boldsymbol{U}$, intersection with regular sets and covering, by Theorem $6.8, \mathcal{L}_{1} \dot{\nabla} \mathcal{L} 2$ has these closure properties. But by definition $\boldsymbol{Z}$ contains all such subsets of $\mathbb{S}$, hence $\mathcal{Z}_{1} \dot{\nabla} \mathcal{Z}_{2}$ is in $\eta$.

By a similar argument we can show that if $\mathcal{L}$ is in $\boldsymbol{n}$ then $\mathcal{L}(p)$ is in $\eta$ for all $p>0$. Hence $\eta$ is an MSW space.

In Corollary 6.9 beginning with an arbitrary language family $\mathcal{L}$ we first formed its closure under $\bullet$, intersection with regular sets and covering to give a collection $\boldsymbol{m}$. Secondly we closed $\boldsymbol{m}$ under $\dot{\forall}$ and extraction to give a new collection $\boldsymbol{n}$ which is an MSW space. We now show that these closure operations need be applied only once in the prescribed order: intersection with regular sets, covering, $\boldsymbol{U}$, extraction and $\dot{V}$. This provides us with a simple means for constructing MSW spaces.

Lemma 6.12

Let $\mathcal{S}$ be an arbitrary language family and $\not{\alpha}$ its closure under $\bullet$. Then $\boldsymbol{S}$ is closed under intersection with regular sets and covering iff $\mathcal{L}$ is so closed.

Proof: Suppose $L$ is in $\mathcal{L}$ and $R$ is an arbitrary regular set. Then $L=L_{1} \dot{\theta} \ldots \dot{\theta} L_{n}$ for some $n \geq 1$ with $L_{i}$ in $\mathcal{S}, 1 \leq i \leq n$. Immediately, $L \cap R=\left(L_{1} \cap R\right) \cup \forall\left(L_{n} \cap R\right)$ and since $L_{i} n R$ is in $\ddot{\mathcal{S}}, 1 \leq \dot{1} \leq n$, it follows that $L \cap R$ is in $\mathcal{L}$, by construction. Since $\mathscr{S} \subseteq \mathcal{L}$ the converse follows immediately. 
Second, suppose for some $L \equiv \Sigma^{*}, L_{(i)} \neq L$ is in $\mathcal{L}$ for infinitely many $i$. We need to show that $L$ is in $\mathcal{L}$.

Since $\Sigma$ has only a finite number of partitions

$\Sigma=\Sigma_{1} \dot{\cup} \Sigma_{2} \dot{U} \ldots \Sigma_{k}$ for $k \geq 1$ and $\Sigma_{j} \neq 0,1 \leq i \leq k$, there exists one partition $\Sigma=\Sigma_{1} \dot{U} \ldots \Sigma_{k}$ say, such that for infinitely many $i$, $L(i)=L_{1, i} \mathbf{U} \ldots \mathcal{U}_{k, i}$ with $L_{j, i} \leq \Sigma_{j}^{*}$ and $L_{j, i}$ in $\mathbb{S}, 1 \leq j \leq k$. Furthermore for this partitition $L_{j, i}=M_{j}(i)$ for $M_{j}=\Sigma_{j}^{*} \cap L$, $1 \leq j \leq k$. But for each $j, 1 \leq j \leq k, M_{j}(i)$ is in $\mathcal{S}$ for infinitely many $i$, hence $M_{j}$ is in $\boldsymbol{S}$ and therefore $L=M_{1} \mathbf{U} \ldots M_{k}$ is in $\mathcal{L}$. Again the converse is immediate.

Eemma 6.13

Let $\boldsymbol{S}$ be an arbitrary language family and $\mathcal{L}$ its closure under covering. If $S$ is closed under intersection with regular sets then $\mathcal{L}$ is so closed.

Proof: Assume that $\boldsymbol{S}$ is closed under intersection with regular sets. Given an arbitrary $L$ in $\mathcal{L}$ and an arbitrary regular set $R$ we need to show that $L \cap R$ is in $\mathcal{L}$. Clearly if $L$ is in $\boldsymbol{S}$ then by assumption $L \cap R$ is in $\boldsymbol{S}$, therefore consider the case $L$ is not in $\boldsymbol{S}$. Now $L(i) \neq L$ is in $\boldsymbol{S}$ for infinitely many $i$ and for these $i$, $L$ (i) $\cap R=L(i) \cap R(i)=(L \cap R)(i)$ is in $S$. But by the construction this implies $L \cap R$ is in $\mathcal{L}$ as required.

Lemma 6.14

Let $M$ be a collection of language families and $\boldsymbol{\eta}$ be its closure under $\boldsymbol{v}$. If $\boldsymbol{m}$ is closed under extraction, then so is $\boldsymbol{n}$.

Proof: Let $\mathcal{L}$ be an arbitrary language family of $\eta$. Then $\mathcal{L}=\mathcal{L}_{1} \dot{v} \ldots \dot{\nabla} \mathcal{L}_{k}$, for some $k>0$ and $\mathcal{L}_{j}$ in $m, 1 \leq j \leq k$. clearly $\mathcal{L}(i)=\mathcal{L}_{1}(i) \dot{V} \ldots \dot{\nabla} \mathcal{L}_{k}(i)$, for a11 $i>0$ and since $m$ is closed under extraction, $\mathcal{L}_{j}(i)$ is in $m, 1 \leq j \leq k$ and hence by construction $\mathcal{L}(i)$ is in $\eta$.

We now combine these three technical lemmas into our main theorem, namely: 
Let $M$ be an arbitrary collection of language families. Close each family $\mathcal{L}$ in $m$ first under intersection with regular sets, second under covering and third under superdisjoint union. close the resulting collection $\hat{m}$ under extraction and then under superdisjoint wedge. Then $\boldsymbol{\eta}$, the resulting collection, is an MSW space.

Proof: By Lemmas $6.12,6.13$ and 6.14 .

For example, let $\boldsymbol{M}=\left\{\left\{\Sigma^{*}: \Sigma\right.\right.$ an alphabet of n symbols $\}$, for some $n \geq 1$. Then $\hat{m}=\{\mathcal{L}\}$ where $\mathcal{L}$ contains a 11 tanguages of the form:

$$
\mathrm{R}=\mathrm{R}_{1} \cup \ldots \dot{\cup} \mathrm{R}_{\mathrm{k}}
$$

for some $k \geq 1$, where the $R_{i}$ are regular sets over disjoint alphabets $\Sigma_{j}$ and $\# \Sigma_{j} \leq n$. Note that no $R$ in $\mathcal{L}$ contains a word $w i t h$ more than $n$ different letters.

closing $\hat{m}$ under extraction gives $m^{\prime}=\left\{\mathcal{L}_{i_{1}}, \ldots,{ }_{q}\right.$ : $\left.1 \leq i_{1}<i_{2}<\ldots<i_{q}, q \geq 0\right\}$, where $\mathcal{L}_{i}, \ldots, i_{q}$ is defined as $\mathcal{L}_{i_{1}}, \ldots, i_{q-1}\left(i_{q}\right)$, that is $\mathcal{L}_{i_{1}}, \ldots, i_{q}$ is obtained by extracting all words of lengths $i_{1}, \ldots, i_{q}$ from $\mathcal{L}$. Finally closing $m$ ' under superdisjoint wedge gives an MSW space $\eta$ by Theorem 6.15 . Notice that the remark about the structure of words in a language $R$ in $\mathcal{L}$ also holds for any word in a language of a language family in $\boldsymbol{\eta}$, hence $\eta$ misses many regular sets.

\section{II.6.4 Two-symbol Grammatical Families}

In this section we are concerned with two-symbol grammar forms and their associated families. As we have already seen the families $\mathcal{Z}(R E G), \mathcal{Z}(L I N)$ and $\mathcal{Z}(C F)$ are two-symbol grammatical. Indeed the grammar forms $G_{1}, G_{2}$ and $G_{3}$ defined by the productions:

$$
\begin{aligned}
& G_{1}: S \rightarrow a ; S \rightarrow a S, \\
& G_{2}: S \rightarrow a ; S \rightarrow a S ; S \rightarrow S a, \\
& G_{3}: S \rightarrow a ; S \rightarrow S S
\end{aligned}
$$

generate the families $\mathcal{L}$ (REG), $\mathcal{L}$ (LIN) and $\mathcal{L}$ (CF), respectively. Furthermore for an arbitrary two-symbol form $G$ the supernormal theorem tells us that $\mathcal{L}_{\mathrm{s}}(G, \Rightarrow)=\mathcal{L}(\mathrm{CF})$ iff $L(G, \Rightarrow)=\mathrm{a}^{+}$and there is a production $S \rightarrow \alpha$ in $G$ such that $\alpha$ contains at least two appearances of $S$. Also $\mathcal{L}_{s}(G, \Rightarrow) \geq \mathcal{L}(R E G)$ iff $\mathcal{L}_{s}(G, \Rightarrow) \geq \mathcal{L}(F I N)$ iff $L(G, \Rightarrow)=a^{+}$. 
Dual to the notion of density are the notions of predecessor and successor, that is for two families $\mathcal{L}_{1}$ and $\mathcal{L}_{2}$ with $\mathcal{L}_{1} \mathcal{L}_{2}$, we say $\mathcal{Z}_{1}$ is a predecessor of $\mathcal{L}_{2}$ or $\mathcal{L}_{2}$ is a successor of $\mathcal{L}_{1}$ if there is no family $\mathcal{L}_{3}$ properly in between.

Within the framework of context-free two-symbol grammatical families, we can observe that $\mathcal{L}$ (CF) is a successor of $\mathcal{L}$ (LIN) and in fact the only successor. For assume that there is a two-symbol form G such that

$$
\mathcal{L}(L I N) \subseteq \mathcal{L}_{\mathrm{s}}(G, \Rightarrow) \subseteq \mathcal{L}(\mathrm{CF}) \text {. }
$$

Now by the observations above $L(G, \Rightarrow)=a^{+}$and either there is a production $S \rightarrow \alpha$ in $G$ with a containing at least two appearances of $S$ or there is not. In the latter case we obtain $\mathcal{L}_{S}(G, \Rightarrow)=\mathcal{L}$ (LIN), and in the former case we have $\mathcal{Z}_{s}(G, \Rightarrow)=\mathcal{Z}(C F)$. Hence there is no G with $\mathcal{L}_{\mathrm{S}}(\mathrm{G}, \Rightarrow)$ properly in between $\mathcal{L}$ (LIN) and $\mathcal{L}(\mathrm{CF})$.

Note that on the other hand $\mathcal{L}$ (LIN) is not the only predecessor of $\mathcal{L}(C F)$. For example, $G$ defined by the productions:

$$
\mathrm{S} \rightarrow \mathrm{aa} ; \mathrm{S} \rightarrow \text { aaa; } \mathrm{S} \rightarrow \mathrm{SS}
$$

has $\mathcal{L}_{s}(G, \Rightarrow) \underset{\ddagger}{\mathcal{L}(C F)}$ and there is no other family (two-symbol family, that is) in between.

Second observe that if $G$ is a two-symbol form such that $\mathcal{Z}_{\mathrm{S}}(G, \Rightarrow) \underset{\ddagger}{\mp}(\mathrm{REG})$, then $\mathcal{L}_{\mathrm{S}}(G, \Rightarrow)$ and $\mathcal{Z}(\mathrm{REG})$ do not form a dense pair. This follows almost immediately, since $L(G, \Rightarrow) \neq a^{+}$, by the remarks above. Let $i \geq 1$ be the smallest integer such that $a^{j}$ is not in $L(G, \Rightarrow)$. Define a new two-symbol form $G_{1}$ by:

$$
S \rightarrow a ; \ldots ; S \rightarrow a^{i-1} ; S \rightarrow a^{i} S ; S \rightarrow a^{2 i}
$$

then $L\left(G_{1}, \Rightarrow\right)=a^{+}-\left\{a^{i}\right\}$, hence

$$
\mathcal{L}_{s}(G, \Rightarrow) \subseteq \mathcal{L}_{s}\left(G_{1} \Rightarrow\right) \underset{\mathcal{L}}{(R E G)}
$$

and $\mathcal{L}(R E G)$ is a successor of $\mathcal{L}_{s}\left(G_{j}, \Rightarrow\right)$.

These preliminary observations lead us to consider the pair $\mathcal{L}$ (REG) and $\mathcal{L}$ (LIN) of two-symbol families. We will demonstrate that these form a dense pair and as a by-product of the proof we will also show that it is decidable whether two linear two-symbol forms are form equivalent.

Since we are only dealing with linear.two-symbol forms in the sequel we can assume that we only have two types of productions: (1) $s \rightarrow a^{i}, i \geq 0$

and

(2) $s \rightarrow a^{i} \mathrm{Sa}^{j}, i, j \geq 0$.

Those of type (1) are terminating productions and those of type (2) are nonterminating productions. Since a type (2) production is uniquely determined by the exponents $i$ and $j$, we will often speak of 
the production $(i, j)$. We can always assume that $i+j>0$ without loss of generality, that is the production $S \rightarrow S$ can always be omitted, without changing the language family.

If $G$ is a linear two-symbol form and $p$ and $q$ are non-negative integers with $p+q>0$, we say that the pair $(p, q)$ is generated by $G$ if for some $n>0$,

$$
S \Rightarrow a^{n p} S a^{n q}
$$

is a derivation according to $G$.

To establish the denseness of the pair ( $\mathcal{L}$ (REG), $\mathcal{L}$ (LIN))

for the two-symbol grammatical families we first need three technical lemmas, which demonstrate some basic properties of pairs $(p, q)$.

\section{Lemma 6.10}

Let $G$ be a linear two-symbol form whose nonterminating productions are $\left(i_{1}, j_{1}\right), \ldots,\left(i_{t}, j_{t}\right)$ where the numbering is chosen so that

$$
\frac{i_{1}}{j_{1}} \leq \cdots \leq \frac{i_{t}}{j_{t}} .
$$

(For $j_{v}=0, \frac{i_{v}}{j_{v}}$ is considered to be $\infty$ ).

Then a pair $(p, q)$ of nonnegative integers with $p+q>0$ is generated by $G$ iff there is a $V$ such that

(3) $\frac{i_{v}}{j_{v}} \leq \frac{p}{p} \leq \frac{i_{v+1}}{j_{v+1}}$.

Proof: if: Assume there is a v satisfying (3). Clearly if one of the inequalities in ( 3 ) is not strict then $(p, q)$ is generated by G. Moreover if $t=1$ this is the only case to consider. Hence consider the case that $t>1$ and both inequalities in (3) are strict. In this case the determinants:

$$
c_{v+1}=\left|\begin{array}{cc}
p & q \\
i_{v} & j_{v}
\end{array}\right|, c_{v}=\left|\begin{array}{cc}
i_{v+1} & j_{v+1} \\
p & q
\end{array}\right|, n=\left|\begin{array}{cc}
i_{v+1} & j_{v+1} \\
i_{v} & j_{v}
\end{array}\right|
$$

are all positive integers. Using the identities

$$
c_{v} u_{v}+c_{v+1} i_{v+1}=n p
$$

and

$$
c_{v} j_{v}+c_{v+7}^{j} v+1=n q
$$


we are able to construct a derivation

$$
\mathrm{S} \Rightarrow \mathrm{a}^{n \mathrm{p}_{\mathrm{Sa}} \mathrm{nq}}
$$

in $G$ by applying $c_{v}$ times the production $\left(i_{v}, j_{v}\right)$ and $c_{v+l}$ times the production $\left(i_{v+1}, j_{v+1}\right)$. Thus $(p, q)$ is generated by $G$.

only if: Assume there is no index v satisfying (3) then either

$$
\frac{p}{q}<\frac{i_{1}}{j_{1}} \text { or } \frac{i_{t}}{j_{t}}<\frac{p}{q} \text {. }
$$

with loss of generality we assume $\frac{p}{q}<\frac{i}{j_{1}}$, since the other alternative can be dealt with by interchanging the roles of $p$ and $q$.

If the pair $(p, q)$ is generated by $G$, there are nonnegative integers $c_{v}, v=1, \ldots, t$ and a positive integer $n$ satisfying

$$
\sum_{v=1}^{t} \quad c_{v} v_{v}^{i}=n p \text { and } \sum_{v=1}^{t} c_{v} j_{v}=n q \text {. }
$$

But this is possible only if $\frac{p}{q} \geq \frac{i}{j_{1}}$, a contradiction.

Therefore $(p, q)$ is not generated by $G$.

\section{Lemma 6.17}

Assume that $F$ and $G$ are linear two-symbol forms and $(p, q)$ is a pair of non-negative integers with $p+q>0$. If $F$ does not generate $(p, q), G$ possesses a production $(p, q)$ and also generates a non-regular language, then $\mathcal{L}_{S}(G, \Rightarrow)$ is not contained in $\mathcal{Z}_{S}(F, \Rightarrow)$.

Proof: Let the nonterminating productions of $F$ be $\left(i_{1}, j_{1}\right), \ldots,\left(i_{t}, j_{t}\right)$ ordered so that

$$
\frac{i_{1}}{j_{1}} \leq \frac{i_{2}}{j_{2}} \leq \ldots \leq \frac{i_{t}}{j_{t}} \text {. }
$$

The assumptions for $G$ imply that there is an $m>0$ such that $S \Rightarrow a^{m}$ in G. By Coroliary II.2.10 we may assume that $S \rightarrow a^{m}$ is a production of $G$.

Now if $F$ has no nonterminating productions, that is $t=0$, then $\mathcal{L}_{\mathrm{s}}(\mathrm{F}, \Rightarrow)$ only contains finite languages. Since $\mathcal{L}_{\mathrm{s}}(G, \Rightarrow)$ contains a non-regular language we have $\mathcal{L}_{s}(G, \Rightarrow) \notin \mathcal{L}_{S}(F, \Rightarrow)$ as claimed. Assume $t \geq 1$. By Lemma 6.16 since $(p, q)$ is not generated by $F$ we may assume $\frac{p}{q}<\frac{i}{j_{1}}$ (the other alternative can be treated symmetrically), in which case $i_{1}>0$. 
Since $G$ generates a non-regular language, there are positive integers $r$ and $s$ such that

$$
S \Rightarrow a^{r} \mathrm{Sa}^{\mathrm{s}}
$$

in $G$. If in the given pair $(p, q)$ we have $p=0$, then we have a derivation

$$
s \Rightarrow a^{r} S a s+q v
$$

in $G$ for a $11 v \geq 0$. Now choose $v$ large enough so that

$$
\frac{r}{s+q v} \leq \frac{i}{j_{l}} \text {, }
$$

this must be possible since $i_{1}>0$. By another application of Corollary II.2.10 we may assume that the production $s \rightarrow a^{r} S a s+q v$ is in $G$, and moreover we can replace $(p, q)$ by $(r, s+q v)$ both of whose components are positive. Again if we have $q=0$ in the original pair $(p, q)$ we could proceed in a similar manner. Thus we may assume by the above argument that both $p$ and $q$ are positive.

Finally, consider the language $L=\left\{a p_{b} m_{a} i q: i \geq 0\right\}$. Clearly $L$ is in $\mathcal{L}_{\mathrm{s}}(G, \Rightarrow)$. We claim it is not in $\mathcal{L}_{\mathrm{s}}(\mathrm{F}, \Rightarrow)$.

Assume that $L$ is in $\mathcal{L}_{S}(F, \Rightarrow)$, then there is an $F^{\prime} \Delta_{S} F$ such

that $L=L\left(F^{\prime} \Rightarrow\right)$. Now $F^{\prime}$ is a linear grammar, since $F$ is linear.

Because $L$ is infinite there must be at least one "looping" nonterminal $A$ in $F^{\prime}$, that is there are derivations:

$S \Rightarrow * u A v, A \Rightarrow+x A y$ and $A \Rightarrow * W$

in $F^{\prime}$, where $u, v, x, y$ and $w$ are terminal words and $x y$ is not the empty word.

Now for a $11 n$, $u x^{n} w y^{n} v$ is in $L$ and since $F^{\prime}$ is an interpretation of $F$ we must have:

$$
\frac{i_{1}}{j_{1}} \leq\left|\frac{x}{y}\right| \leq \frac{i_{t}}{j_{t}}
$$

However, $x y$ must be in $a^{+}$and since $u x^{n} w y^{n} v$ is in $L$ for all $n \geq 0$, then $\left|\frac{x}{y}\right|=\frac{p}{q}$. Thus we have obtained a contradiction since we assumed $\frac{p}{q}<\frac{i}{j_{1}}$. Hence $L$ is not $\mathcal{L}_{s}(F, \Rightarrow)$ and therefore $\mathcal{L}_{s}(G, \Rightarrow)$ is not contained in $\mathcal{L}_{s}(F, \Rightarrow)$.

Lemma 6.18

Let $F$ be a linear two-symbol form and $S \rightarrow a^{p} a^{q}$ be a production in $F$, where $p+q>0$. Construct $G$ from $F$ by taking into $G$ : 
(i) all productions in $F$ apart from $S \rightarrow a^{p} S_{a}{ }^{q}$,

(ii) the production $S \rightarrow a^{p n_{S a}}{ }^{q n}$, for some $n \geq 2$, and

(iii) sufficient productions of the form $s \rightarrow a^{i}, i>0$ such that $L(F, \Rightarrow)=L(G, \Rightarrow)$.

Then $F$ and $G$ are form equivalent.

Proof: Clearly $\mathcal{L}_{S}(G, \Rightarrow) \subseteq \mathcal{L}_{S}(F, \Rightarrow)$, by the simulation lemma. Hence we only need consider the reverse inclusion $\mathcal{L}_{s}(F, \Rightarrow) \subseteq \mathcal{L}_{s}(G, \Rightarrow)$. Let $F^{\prime}=\left(V, \Sigma, P^{\prime}, S^{\prime}\right)$ be an arbitrary interpretation of $F, F^{\prime} \frac{S}{S} F(\mu)$. We demonstrate that there is an interpretation $G^{\prime} \underset{S}{\Delta} G$ with $L\left(G^{\prime}, \Rightarrow\right)=L\left(F^{\prime} \Rightarrow\right)$ to complete the proof.

In the case that both $S \rightarrow a^{i} S$ and $S \rightarrow S a^{j}$ are in $F$ for some $i, j>0$, then by Theorem 4.24 we have $\alpha_{s}(F, \Rightarrow)$ equals the family of linear languages whose length set is contained in $L S(F, \Rightarrow)$. This same observation holds for $G$ also, since either both $p$ and $q$ are non-zero in which case $S \rightarrow a^{i} S$ and $S \rightarrow S a^{j}$ are in $G$ or one of $p$ and $q$ is zero, in which case either $S \rightarrow a^{i} S$ and $S \rightarrow S a^{n j}$ or $S \rightarrow a^{n i} S$ and $S \rightarrow S a^{j}$ are in G. In all cases $\mathcal{L}_{\mathrm{S}}(\mathrm{G}, \Rightarrow)=\mathcal{L}_{\mathrm{S}}(\mathrm{F}, \Rightarrow)$.

Returning to $F^{\prime}$ we next observe that if $P^{\prime}$ contains no interpretation of $S \rightarrow{ }^{P} S a^{q}$ then it is trivially an interpretation of $G$ and therefore $L\left(F^{\prime} \Rightarrow\right)$ is in $\mathcal{L}_{s}(G, \Rightarrow)$. So we assume that $F^{\prime}$ does indeed contain some interpretations of $S \rightarrow{ }^{p}{ }_{S a}{ }^{q}$.

We will for the purposes of this proof consider a production $A \rightarrow U B V$ an $(i, j)$ production if it is an image of a production $S \rightarrow a^{i} S^{j}$.

The idea behind the construction of $G^{\prime}$ is as follows. For any derivation $D$ in $F^{\prime}$ the corresponding derivation $D^{\prime}$ in $G^{\prime}$ will correspond exactly until the first appearance of a $(p, q)$ production in $D, A_{0} \rightarrow u_{1} A_{1} v_{1}$, say, since $G$ ' cannot contain a $(p, q)$ production. So we examine the production applied to $A_{T}$ in $D$. There are now three possibilities:

(i) The production applied to $A_{1}$ is terminating, $A_{1}+w$ say. In this case $D$ also terminates and in $D^{\prime}$ we apply the production $A_{0} \rightarrow u_{1} w v_{1}$ to $A_{0}$. We may assume that this production is in $G^{\prime}$, since the derivation $S \Rightarrow{ }^{*} a^{i} a^{j} a^{k}$ is in $F$ where $i=\left|u_{j}\right|$, $j=|w|$ and $k=\left|v_{1}\right|$ and moreover $F$ and $G$ generate the same language.

(ii) The production applied to $A_{1}$ is nonterminating and is not a $(p, q)$ production. Let it be $A_{1} \rightarrow u_{2} A_{2} v_{2}$, then in $D^{i}$ we apply the production $A_{0} \rightarrow x_{1}\left[x_{2} A_{2} y_{2}\right] y_{1}$ 
to $A_{0}$, where $\left|x_{1}\right|=\left|u_{2}\right|,\left|y_{1}\right|=\left|v_{2}\right|,\left|x_{2}\right|=p$, $\left|y_{2}\right|=q, x_{1} x_{2}=u_{1} u_{2}, y_{2} y_{1}=v_{2} v_{1}$, and $\left[x_{2} A_{2} y_{2}\right]$

is a new nonterminal. Note that this production is an interpretation of the same production as $A_{1} \rightarrow u_{2} A_{2} v_{2}$. The new nonterminals carry along terminal words to be deposited as soon as possible. One situation in which they can be deposited is when a terminating production is met, the other situation is when terminal words of sufficient length have been accumulated in the nonterminals.

(iii) The production applied to $A_{7}$ is nonterminating and is also a $(p, q)$ production, $A_{1} \rightarrow u_{2} A_{2} \dot{v}_{2}$ say. In this case we examine the production applied to $A_{2}$ in $D$. Again we have three cases to consider, case ( $i$ ) is the same as above and case ( $i i$ ) has a minor modification namely $\left|x_{2}\right|=2 p,\left|y_{2}\right|=2 q$. In case ( $i i i$ ) we proceed in the same manner, however when we find $n(p, q)$ productions $A_{0} \rightarrow u_{1} A_{1} v_{1}, \ldots, A_{n-1} \rightarrow u_{n} A_{n} v_{n}$, then we replace them by $A_{0} \rightarrow u_{1} \ldots u_{n} A_{n} v_{n} \ldots v_{1}$ in $D^{\prime}$. This we can do since $\left|u_{1} \ldots u_{n}\right|=n p,\left|v_{n} \ldots v_{1}\right|=n q$ and $G$ has an $(n p, n q)$ production.

It should be clear how to modify the above procedure when dealing with one of the new nonterminals [xAy] in $D^{\prime}$, namely, the case analysis is the same except that: in case ( $i) x$ and $y$ must also be deposited; in case ( $i i)$ a new nonterminal will carry terminal information of lengths $|x|+p$ and $|y|+q$ (unless $|x|+p=n p$ in which case introduce an $(n p, n q)$ production to deposit the terminal words before continuing); in case (iii) terminal information will be accumulated unless its length equals np and nq, when it is deposited. This informal description of the construction of $G$ ' we now formalize.

Let the nonterminals of $G$ ' be triples of the type

$$
\text { [xAy] }
$$

where $x$ and $y$ are in $\Sigma^{*},|x|=r p,|y|=r q$ for some $r, 0 \leq r<n$ and $A$ is in $V-\Sigma$. Let $\left[S^{\prime}\right]$ be the sentence symbol of $G^{\prime}$. Essentially a nonterminal [A] corresponds exactly to a nonterminal $A$ in $F^{\prime}$ and a nonterminal [xAy], $x y \neq \lambda$ contains the accumulated terminal information as mentioned above.

For each nonterminal $A_{0}$ in $F^{\prime}$ and each nonterminal [xA $y$ ] in $G^{\prime}$, where $|x|=r p$ and $|y|=r q$ for some $r, 0 \leq r<n$, define the following sets of derivations: 


$$
\begin{aligned}
C_{r}\left(A_{0}\right)= & \left\{A_{0} \Rightarrow u_{1} A_{1} v_{1} \Rightarrow \ldots \Rightarrow u_{1} \ldots u_{s} A_{s} v_{s} \ldots v_{1} \text { in } F^{\prime}: s \geq 1,\right. \\
& A_{i} \rightarrow u_{i+1} A_{i+1} v_{i+1} \text { is a }(p, q) \text { production, } 0 \leq i<s \\
& \text { and } s+r=n\}, \\
D_{r}\left(A_{0}\right)= & \left\{A_{0} \Rightarrow u_{1} A_{1} v_{1} \Rightarrow \ldots \Rightarrow u_{1} \ldots u_{s+1} A_{s+1} v_{s+1} \ldots v_{1}\right. \text { in } \\
& F^{\prime}: s \geq 0, A_{i} \rightarrow u_{i+1} A_{i+1} v_{i+1} i s \text { a }(p, q) \text { production, } \\
& 0 \leq i<s, s+r<n \text { and } A_{s} \rightarrow u_{s+1} A_{s+1} v_{s+1} \text { is not } \\
& a(p, q) \text { production\}, } \\
T_{r}\left(A_{0}\right)= & \left\{A_{0} \Rightarrow u_{1} A_{1} v_{1} \Rightarrow \ldots \Rightarrow u_{1} \ldots u_{s} A_{s} v_{s} \ldots v_{1} \Rightarrow\right. \\
& u_{1} \ldots u_{s} v_{s} \ldots v_{1} \text { in } F^{\prime}: s \geq 0, A_{i} \rightarrow u_{i+1} A_{i+1} v_{j+1} \\
& i s \text { a }(p, q) \text { production, } 0 \leq i<s \text { and } r+s<n\} .
\end{aligned}
$$

These three sets exhaust the possible derivations from $A_{0}$ which involve an initial, possible empty, sequence of $(p, q)$ productions when the terminal words $x$ and $y$ accumulated so far are of length $r p$ and $r q$ respectively. We can now define the productions of $G^{\prime}$ as follows: For all nonterminals $\left[x A_{0} y\right]$ in $G^{\prime}$ where $|x|=r p$ and $|y|=r q$ for some $r, 0 \leq r<n$ we include the following productions:

(i) $\left[x A_{0} y\right] \rightarrow x u_{1} \ldots u_{s}\left[A_{s}\right] v_{s} \ldots v_{1} y$, where the derivation

$$
A_{0} \Rightarrow u_{1} \ldots u_{s} A_{s} v_{s} \ldots v_{1} \text { is in } c_{r}\left(A_{0}\right) \text {, }
$$

(ii) $\left[x A_{0} y\right] \div w\left[x^{\prime} A_{S+1} y^{\prime}\right] z$, where the derivation

$A_{0} \Rightarrow u_{1} \ldots u_{s+1} A_{s+1} v_{s+1} \cdots v_{1}$ is in $D_{r}\left(A_{0}\right)$,

$w x^{\prime}=x u_{1} \ldots u_{s+1}, y^{\prime}=v_{s+1} \cdots v_{1} y,|w|=\left|u_{s+1}\right|$

and $|z|=\left|v_{s+1}\right|$,

(iii) $\left[x A_{0} y\right] \rightarrow x u_{1} \ldots u_{s} w v_{s} \ldots v_{7} y$, where the derivation

$A_{0} \Rightarrow+u_{1} \ldots u_{s} w v_{s} \ldots v_{1}$ is in $T_{r}\left(A_{0}\right)$.

Note that productions of type (i) are possible to obtain from $G$ since $G$ has the production $S \rightarrow a^{n p} S a^{n q}$, those of type (ii) since

$A_{S} \rightarrow u_{s+1} A_{s+1} v_{s+1}$ is not a $(p, q)$ production and those of type (iii)

since the language of $G$ equals that of $F$.

Hence we have constructed $a G^{\prime} \underset{S}{\Delta} G$ and the motivation given above for the construction implies that $L\left(G^{\prime}, \Rightarrow\right)=L\left(F^{\prime}, \Rightarrow\right)$. A detailed proof of this is left to the reader.

Therefore we have shown that $\mathcal{L}_{S}(G, \Rightarrow)=\mathcal{L}_{S}(F, \Rightarrow)$ as desired.

We are now able to establish the required theorem, namely:

\section{Theorem 6.19}

The pair ( $\mathcal{L}(R E G), \mathscr{L}(L I N))$ is dense. 
Proof: Assume that $G_{1}$ and $G_{2}$ are two-symbol forms such that $\mathcal{L}(R E G) \subseteq \mathcal{L}_{\mathrm{s}}\left(G_{1}, \Rightarrow\right)$ 呆 $\mathcal{L}_{\mathrm{s}}\left(G_{2}, \Rightarrow\right) \subseteq \mathcal{L}($ LIN $)$.

Note that $L\left(G_{1}, \Rightarrow\right)=L\left(G_{2}, \Rightarrow\right)=a^{+}$must hold. Let $\left(i_{1}, j_{1}\right), \ldots,\left(i_{t}, j_{t}\right)$ be the nonterminating productions of $G_{1}$, ordered by increasing ratios as above. By the previous 1emma, Lemma 6.18 , and by the assumptions for $G_{1}$ and $G_{2}$ we must have a $(p, q)$ production in $G_{2}$ such that $(p, q)$ is not generated by $G_{1}$ (otherwise $G_{1}$ and $G_{2}$ are form equivalent, a contradiction).

By Lemma 6.16 we either have

$$
\frac{p}{q}<\frac{i}{j_{1}} \text { or } \frac{i_{t}}{j_{t}}<\frac{p}{q} \text {. }
$$

As before we assume the former holds since the latter can be treated symmetrically.

Now each of $\left(i_{k}, j_{k}\right), 1 \leq k \leq t$ are generated by $G_{2}$ (Lemma 6.17 ). Therefore for some $n>0$

$$
s \Rightarrow a^{n i}{ }^{n} a^{n j} 1
$$

is a derivation in $G_{2}$.

Now let $G_{3}$ be the two-symbol form obtained from $G_{1}$ by adding the production

$$
S \rightarrow a^{n i_{1}+p} s a^{n j_{1}+q}
$$

Clearly $\mathscr{L}_{\mathrm{s}}\left(\mathrm{G}_{1}, \Rightarrow\right) \subseteq \mathcal{L}_{\mathrm{s}}\left(\mathrm{G}_{3}, \Rightarrow\right)$ and since $\frac{\mathrm{p}}{\mathrm{q}}<\frac{\mathrm{i}_{1}}{j_{1}}$ we also have $\frac{n i_{1}+p}{n j_{1}+q}<\frac{i_{1}}{j_{1}}$, thus the inclusion is proper. On the other hand $s \Rightarrow a^{n i} I_{S a}{ }^{n j} \Rightarrow_{1} a^{n i}{ }^{+p} S a^{n j}{ }^{+q}$ is a derivation in $G_{2}$, hence $\mathcal{L}_{s}\left(G_{3}, \Rightarrow\right) \subseteq \mathcal{L}_{s}\left(G_{2}, \Rightarrow\right)$. Now $\frac{p}{q}<\frac{n i_{1}+p}{n j_{1}+q}<\frac{i_{1}}{j_{1}}$, thus $(p, q)$ is not generated by $G_{3}$ and the inclusion is once again a proper one.

To summarize: we have constructed a $G_{3}$ such that

$$
\mathcal{L}_{s}\left(G_{1}, \Rightarrow\right) \mathcal{L}_{s}\left(G_{3},\right) \subsetneq \mathcal{L}_{s}\left(G_{2}, \Rightarrow\right)
$$

for arbitrary $G_{1}$ and $G_{2}$ satisfying the conditions of the theorem. Therefore $(\mathcal{L}(\mathrm{REG}), \mathcal{L}(\mathrm{LIN}))$ is dense.

We now use the techniques developed above to give a surprising decidability result.

\section{Theorem 6.20}

Given two-symbol forms $G_{1}$ and $G_{2}$, where $G_{1}$ is a linear two-symbol form. Then it is decidable whether or not $\mathcal{L}_{s}\left(G_{1}, \Rightarrow\right)=\mathcal{L}_{s}\left(G_{2}, \Rightarrow\right)$. 
Proof: Without loss of generality we may assume both $G_{1}$ and $G_{2}$ contain a terminating production. First decide whether or not $L\left(G_{1}, \Rightarrow\right)=L\left(G_{2}, \Rightarrow\right)$. Since $L\left(G_{i}, \Rightarrow\right)$ is a regular language, $i=1,2$, this can be accomplished. If $L\left(G_{1}, \Rightarrow\right) \neq L\left(G_{2}, \Rightarrow\right)$ then $G_{1}$ and $G_{2}$ cannot be form equivalent. On the other hand if equality holds we can consider the following possibilities.

(i) $G_{2}$ is nonlinear. Clearly $G_{1}$ and $G_{2}$ cannot be form equivalent.

(ii) $G_{1}$ and $G_{2}$ are either left or right 1 inear. In this case both generate all regular languages whose length sets are contained in those of $G_{1}$ and hence of $G_{2}$. Therefore they are form equivalent in this case.

(iii) Neither (i) nor (ii) hold. Hence both $G_{1}$ and $G_{2}$ are linear. Moreover (a) both are finite, (b) both are properly linear or (c) neither (a) nor (b) hold. In case (a) $G_{1}$ and $G_{2}$ are form equivalent and in (c) they cannot be form equivalent, since either one is finite and the other infinite or one is subregular and the other is properly linear. This only leaves case (b), but by the previous results $G_{1}$ and $G_{2}$ are form equivalent iff for each production $(p, q)$ in $G_{1}(p, q)$ is generated by $G_{2}$ and vice versa. This test is effective by the previous lemmas and therefore form equivalence is decidable.

In Theorem 6.20 the restriction that $G_{1}$ be linear cannot be removed at the present time. However the following conjecture, which we strongly believe to hold, would enable its removal to be accomplished.

\section{Conjecture:}

Assume $G$ is a non-linear two-symbol form. Then every contextfree language whose length set is contained in $L S(G, \Rightarrow)$ is in $\mathcal{L}_{S}(G, \Rightarrow)$. 